
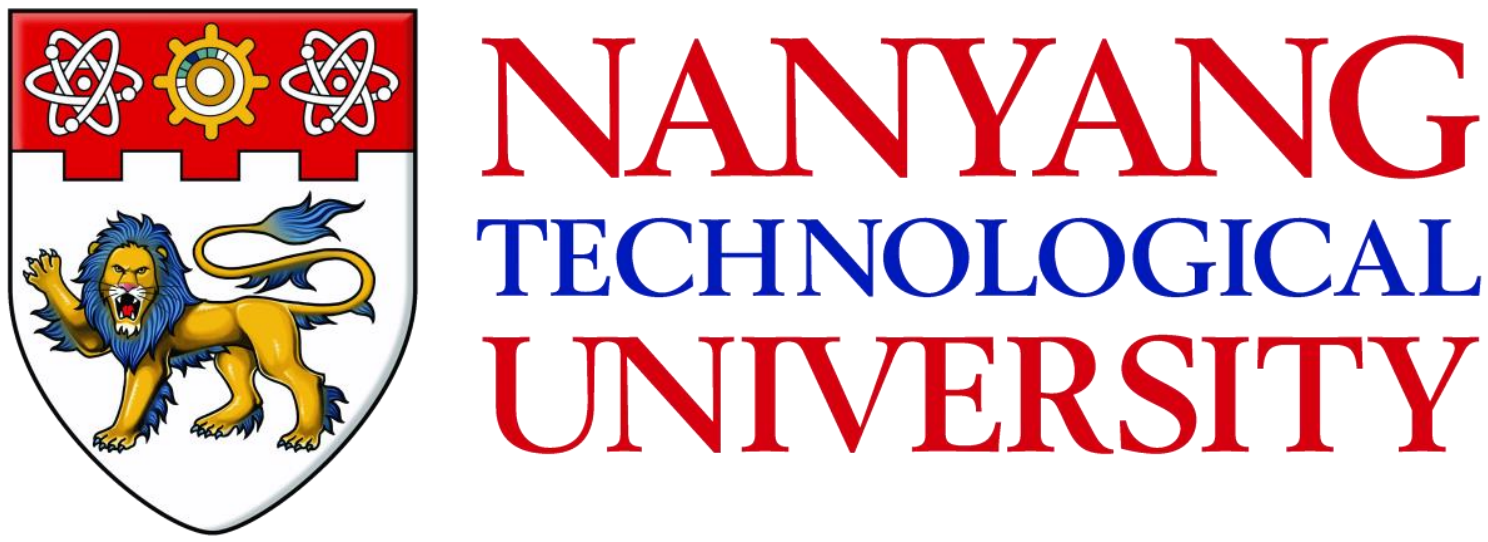

MAGNETIZATION DYNAMICS OF DOMAIN WALL BASED DEVICES FOR LOGIC AND MEMORY APPLICATIONS

PANKAJ SETHI

SCHOOL OF PHYSICAL AND MATHEMATICAL SCIENCES 


\section{MAGNETIZATION DYNAMICS OF DOMAIN WALL BASED DEVICES FOR LOGIC AND MEMORY APPLICATIONS}

\section{PANKAJ SETHI}

SCHOOL OF PHYSICAL AND MATHEMATICAL SCIENCES

A thesis submitted to Nanyang Technological University in partial fulfilment of the requirements for the degree of

Doctor of Philosophy 


\section{Acknowledgements}

This thesis is a result of endless hard work put in not just by me but many other people involved directly or indirectly with my research work. Firstly, I would like to express my deep gratitude to my Ph. D. supervisor, Assoc. Prof. Lew Wen Siang. He has provided invaluable guidance, encouragement and feedback throughout the period of my candidature. He gave me full freedom and independence to pursue my research interests. I am thankful for his ready availability for discussions and insightful suggestions which have helped me improve my writing and presentations skills. Besides my supervisor, I would like to thank my thesis advisory committee members, Assoc. Prof. Sun Handong and Assoc. Prof. Wang Junling for their support and feedback.

I am also grateful to my co-supervisors from Data Storage Institute (DSI), Dr. Leong Siang Huei and Dr. Chen Yunjie for letting me use the experimental facilities at DSI. They assigned and supervised me on hard-disk media projects which helped to broaden my knowledge in magnetism. They monitored my progress through regular meetings and gave constructive feedback. These discussions greatly helped me in my research work.

I would like to thank Dr. Sarjoosing Goolaup for helping me during the initial phase of my $\mathrm{Ph}$. D. He helped me develop some of the experimental skills along with teaching me micromagnetic simulations. He provided valuable suggestions and solutions to some of the research problems. I am also thankful to Dr. Chandrasekhar Murapaka for providing great support both as a colleague and a friend. He taught me majority of the experimental techniques (electron beam lithography, ion-milling, magnetic force microscopy). He is an extremely approachable and skilful person. I thoroughly enjoyed our discussions together which led us to solve some of the most challenging problems. I am grateful to Dr. Chinkhanlun Guite for helping build the electrical measurement set-up which has been of immense use. I would also 
like to thank Mr. Gerard Joseph Lim and Mr. Farhan Nur Kholid for helping to grow thin film stacks using sputtering technique. Gerard has been extremely helpful in troubleshooting majority of the problems with lab equipment, his contribution is immense. I am also thankful to Mr. Weiliang Gan for setting up the Kerr microscopy imaging technique in our lab and helping to perform some of the crucial measurements. I also acknowledge Mr. Tan Funan for helping in the Labview programming and troubleshooting.

I had great fun working in the Spintronics Device Lab and my stay was made more enjoyable by my lab mates. I am thankful to all my lab colleagues who have been working with me for the past four years. I would like to make special mention of my friends Ramu and Sachin. They have provided me with immense support both professionally and personally for which I would be forever grateful.

Lastly, but most importantly, I would like to thank my family members who are the pillar of my strength. My father for believing in me, more than I did, and motivating me through the tough period. My mother for always caring and emotionally supporting me. My sister, Mira, for being there for me always and without whose support life would be difficult to imagine. My brother in-law Pankaj for his kind encouragement and support. My grandmother and uncle whose blessings have helped me clear most difficult obstacles. 


\section{Table of Contents}

List of Figures

(viii)

List of Tables

(xiii)

Summary

(xiv)

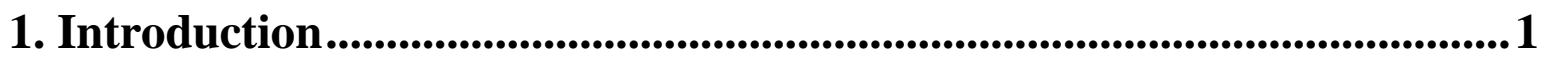

1.1 Basic Theory of Ferromagnetism and Domain formation........................................... 3

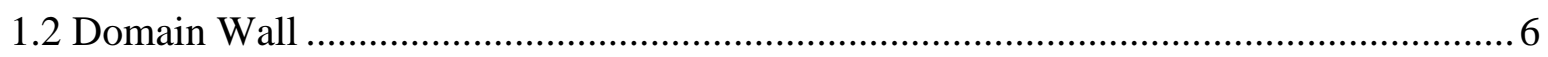

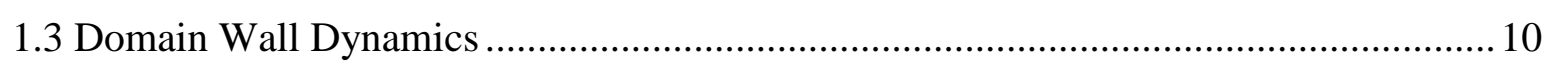

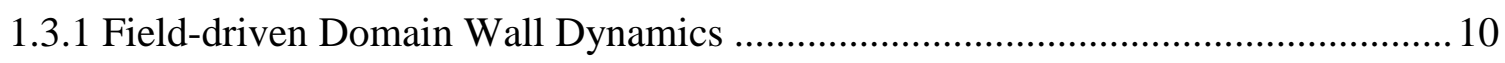

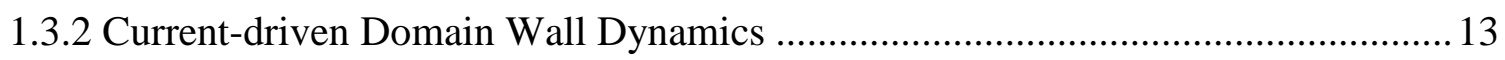

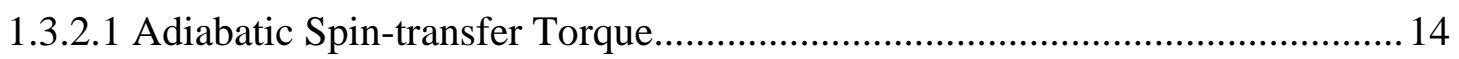

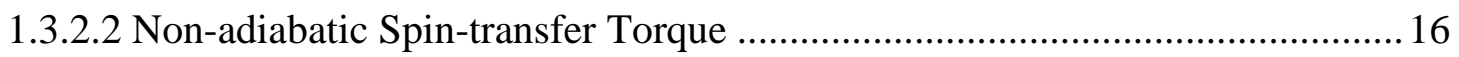

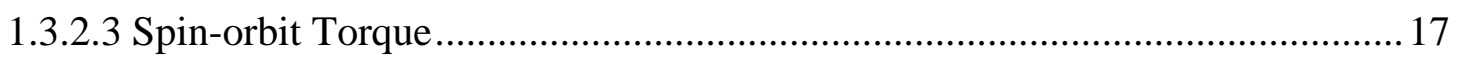

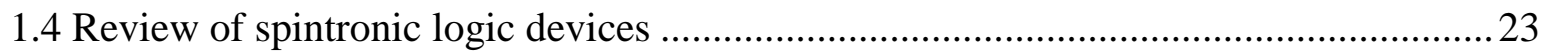

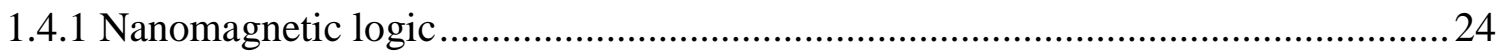

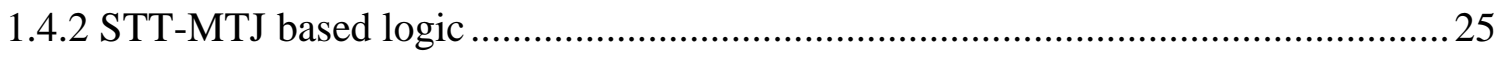

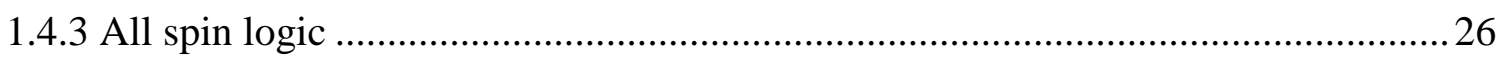

1.5 Existing memory and logic schemes based on domain-wall dynamics ........................29

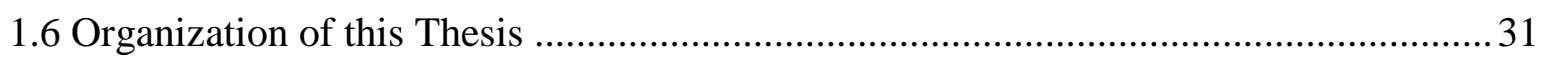

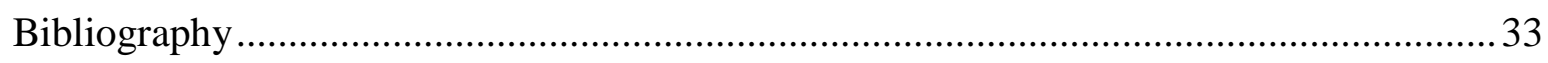

2. Experimental Methods .............................................................................38

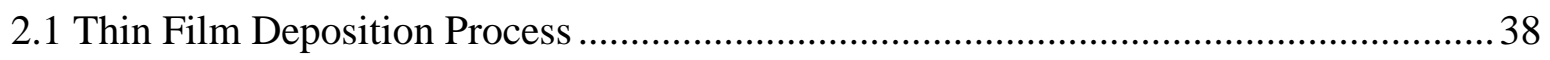

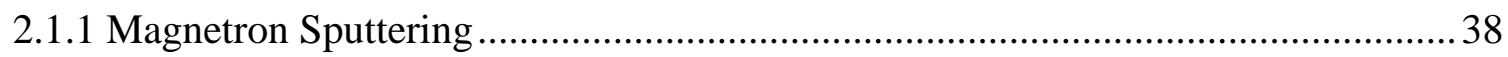

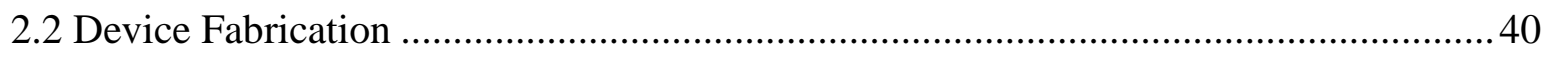

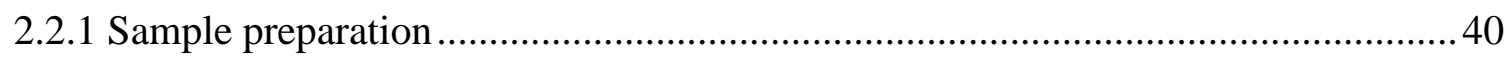

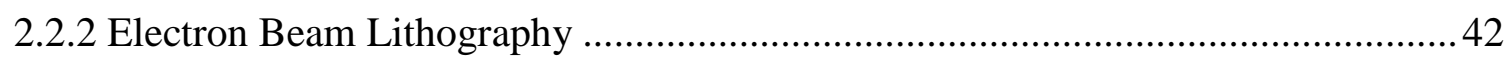

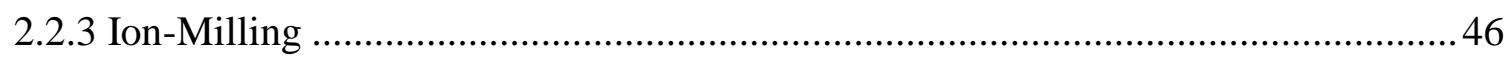

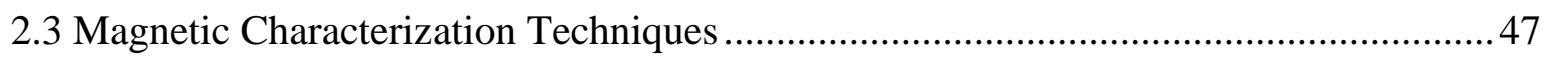


2.3.1 Magnetic Force Microscopy

2.3.2 Alternating Gradient Force Magnetometry ...................................................... 49

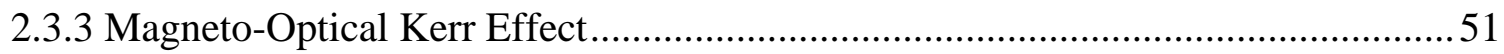

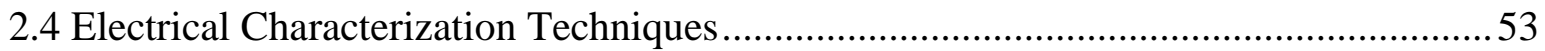

2.4.1 Domain wall nucleation, detection and driving procedure....................................54

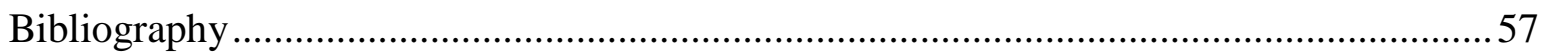

\section{Controlled Motion of Domain-Wall in Network Nanostructures with} In-Plane Magnetization Anisotropy ...........................................................59

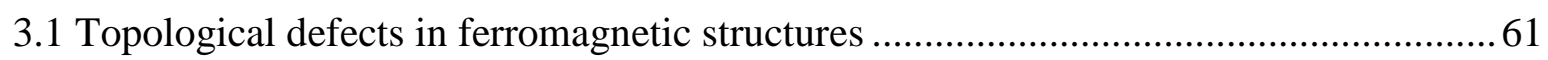

3.2 Evolution of domain-wall at the bifurcation - a micromagnetic study .......................... 64

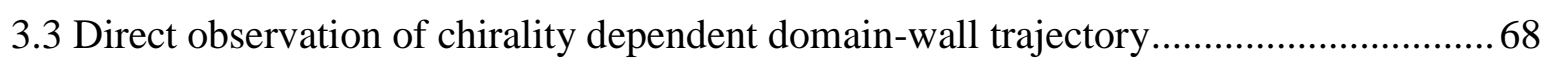

3.4 Observation of Walker breakdown and stochastic trajectory of domain wall in network

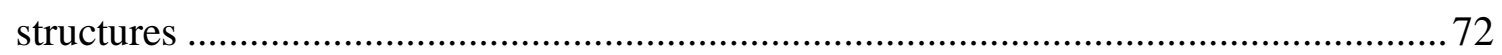

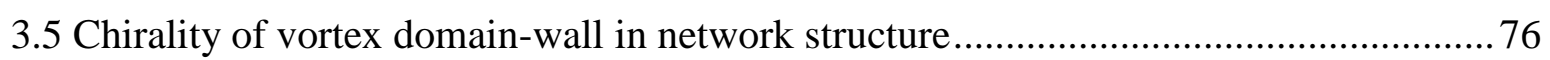

3.6 Geometrical asymmetry to control trajectory of domain wall in network structure .......77

3.6.1 Constrained motion of domain-wall along the upper branch ................................ 78

3.6.2 Constrained motion of domain-wall along the lower branch ............................... 81

3.6.3 Evolution of vortex domain-wall at the bifurcation of asymmetric structure ..........84

3.6.4 Potential energy barrier at the bifurcation as a function of geometrical asymmetry

3.6.5 Effect of initial vortex core polarity on the domain-wall trajectory ........................ 89

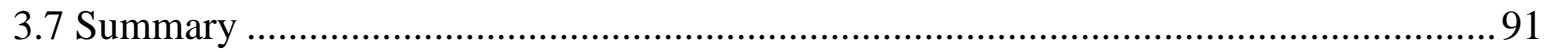

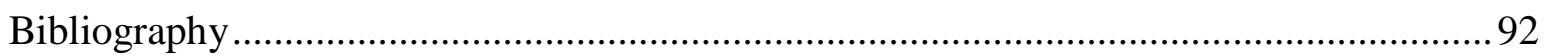

\section{Programmable Logic via Gate Controlled Motion of Domain Wall in} Magnetic Network Structure ..................................................................94

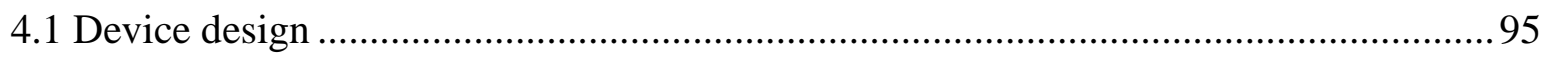

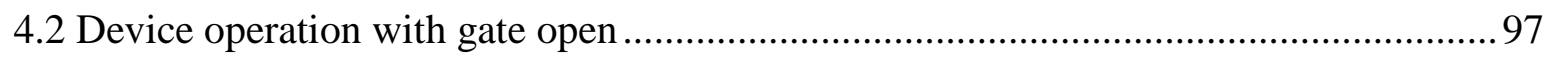

4.3 Transformation of vortex to transverse domain wall and gate overlap extent ..............99

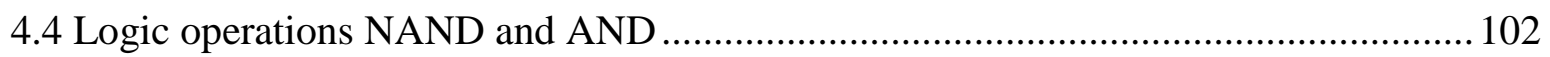

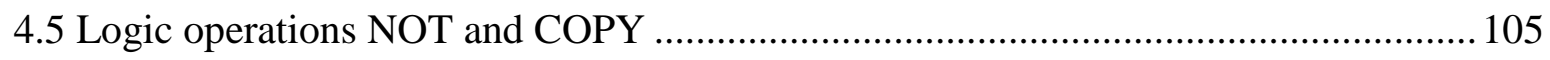

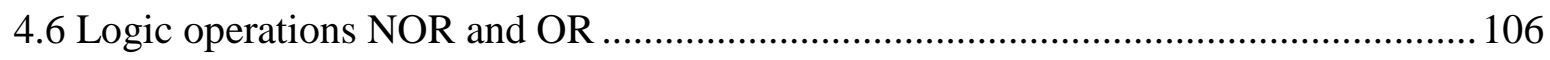

4.7 Verification of logic functionality using magnetoresistance measurements ................ 109 


\section{In-plane Current Induced Stochastic Domain-Wall Nucleation in} Perpendicular Magnetic Anisotropy Nanowires

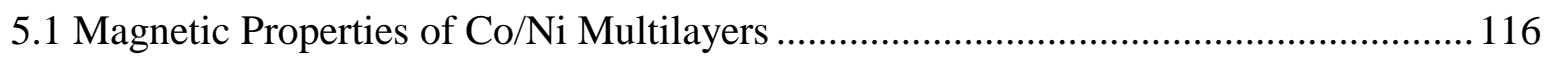

5.2 Field Induced Domain Wall Motion ........................................................................ 122

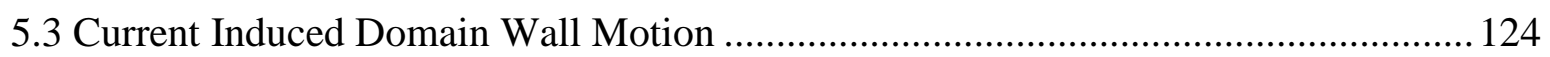

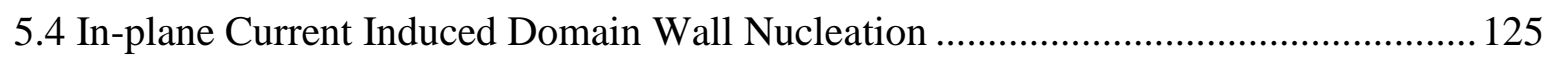

5.4.1 Detection of domain wall at the Hall cross by using Kerr imaging ...................... 128

5.4.2 Back and forth motion of domain walls by spin-transfer torque nucleated at the

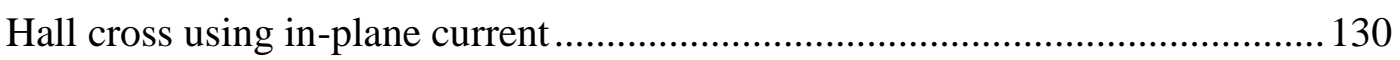

5.4.3 Effect of pulse width modulation on domain wall nucleation process.................. 131

5.4.4 In-plane current induced domain wall nucleation in device with relatively larger

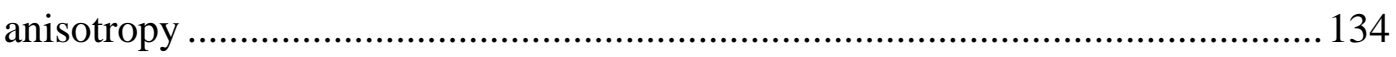

5.4.5 In-plane current injection in nanowire without Hall cross .................................. 135

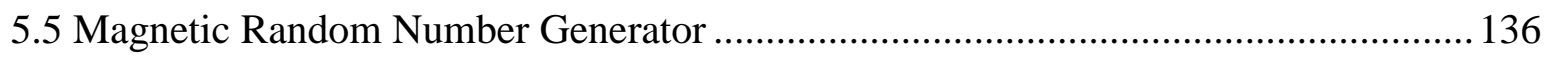

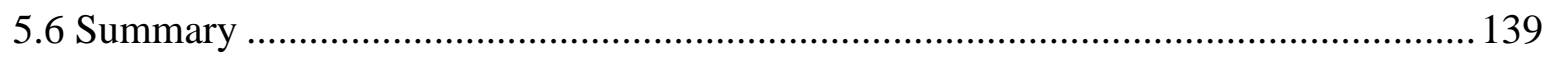

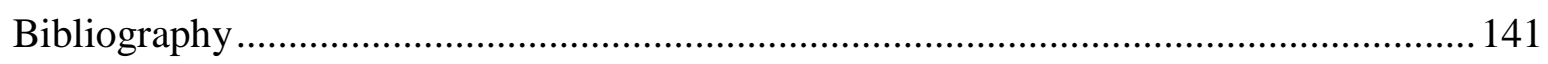

\section{Spin-Orbit Torque Induced Domain-Wall Dynamics in Perpendicular} Magnetic Anisotropy Structures ..............................................................143

6.1 Experimental techniques and comparison of thermal stability for single and double

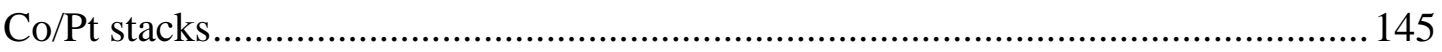

6.2 Magnetization and spin distribution of $\mathrm{Co} / \mathrm{Pt}$ double stack device ............................ 147

6.3 Harmonic Hall measurements and estimation of spin Hall angle ............................... 149

6.4 Spin-orbit torque driven domain wall dynamics in $\mathrm{Co} / \mathrm{Pt}$ multilayers ........................ 158

6.4.1 Effect of Ta capping thickness on domain wall velocity ...................................... 160

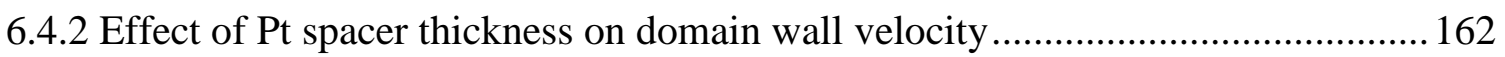

6.5 Estimation of Dzyaloshinskii-Moriya interaction strength using domain wall creep

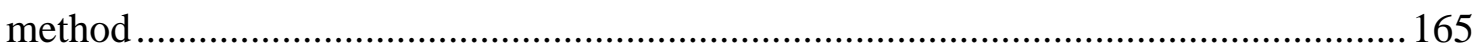

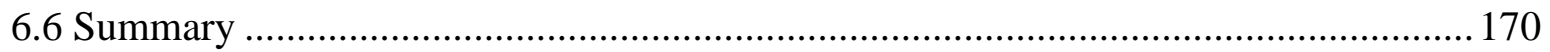

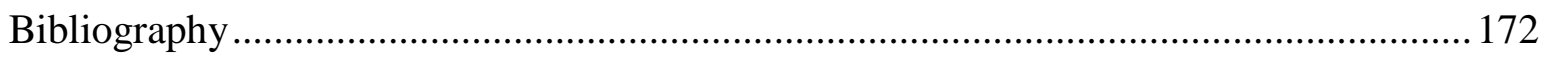


7. Conclusions and Outlook

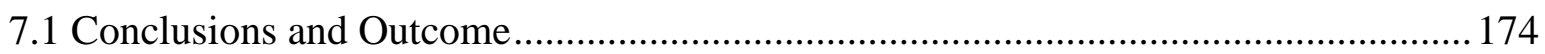

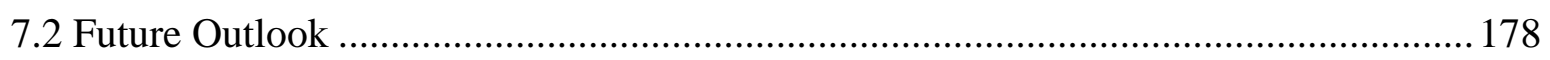

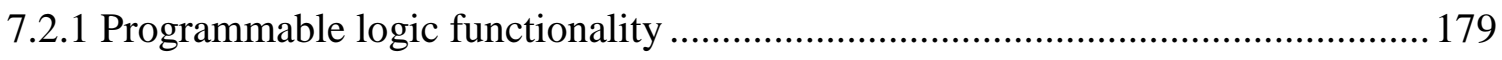

7.2.2 Spin-orbit torque induced domain-wall motion assisted by Dzyaloshinskii-Moriya

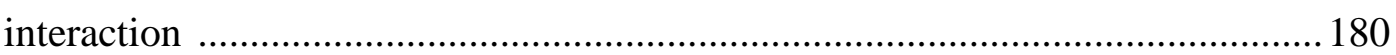

7.2.3 Voltage controlled magnetization switching and domain wall motion ................. 182

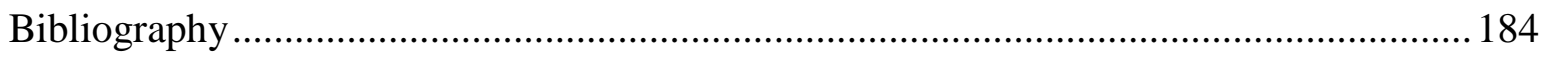

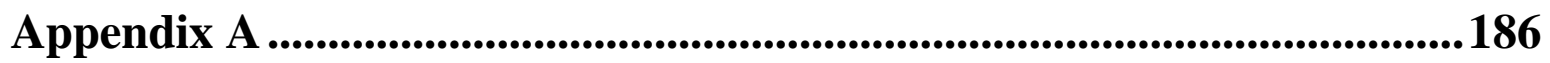

A.1 X-ray photoelectron spectroscopy of Co/Pt double stack ......................................... 186

A.2 Determination of anisotropy constant of $\mathrm{Co} / \mathrm{Pt}$ double stack ..................................... 188

A.3 Effect of in-plane field on domain wall motion and structure.................................. 190

A.4 Domain wall velocities: Down-up chirality ........................................................ 191

A.5 Stray field of permanent bar magnet .................................................................. 193

A.6 Calculation of Dzyaloshinskii-Moriya interaction energy ...................................... 194

A.7 Reliability and endurance of domain wall based devices ........................................ 195

A.8 Geometrical asymmetry induced by steepness in branches ..................................... 195

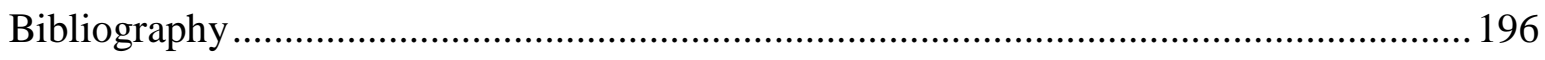

List of Publications ..............................................................................199 


\section{List of Figures}

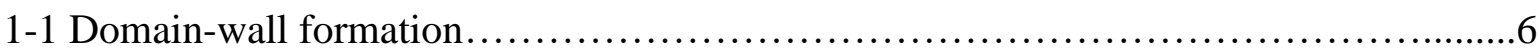

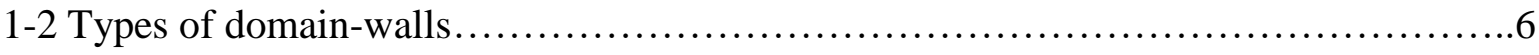

1-3 Demagnetization energy in Néel walls....................................... 8

1-4 Domain-walls in in-plane magnetization materials...............................9

1-5 Precession and damping of spins in presence of external magnetic field...............11

1-6 Field driven domain-wall dynamic in in-plane magnetized nanostrips................12

1-7 Plot of velocity versus magnetic field to illustrate Walker breakdown.................12

1-8 Field driven domain-wall dynamic in out-of-plane magnetized nanostrips............13

1-9 Schematic illustrating spin-transfer torque......................................

1-10 Current driven domain-wall dynamic in out-of-plane magnetized nanostrip through

adiabatic STT ................................................................. 15

1-11 Current driven domain-wall dynamic in out-of-plane magnetized nanostrip through

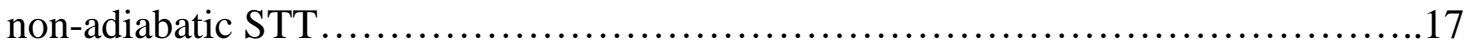

1-12 Schematic illustrating Rashba effect........................................

1-13 Schematic illustrating spin-Hall effect.....................................20

1-14 Schematic illustrating Dzyaloshinskii-Moriya interaction vector.....................20

1-15 Schematic depicting sense of rotation of chiral Néel walls .......................22

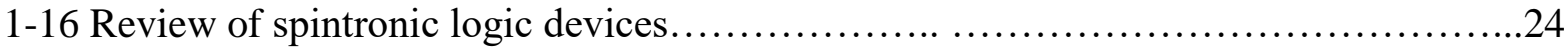

1-17 Existing domain-wall based memory and logic schemes........................ 30

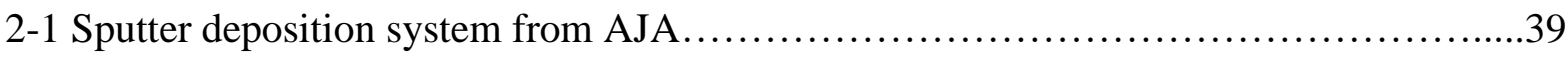

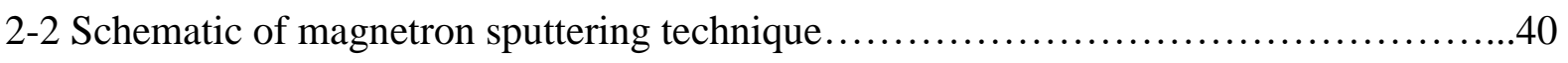

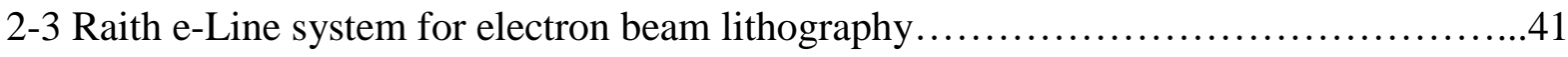

2-4 Schematic of electron beam lithography technique...............................42 


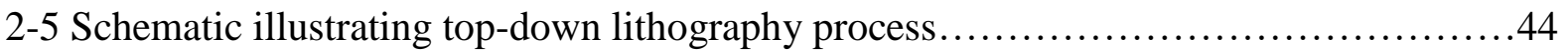

2-6 Schematic illustrating bottom-up lithography process............................45

2-7 Ion-milling system from AJA ........................................... 46

2-8 Schematic illustrating magnetic force microscopy imaging $\ldots \ldots \ldots \ldots \ldots \ldots \ldots \ldots \ldots \ldots . .48$

2-9 Schematic illustrating alternate gradient force magnetometry set-up..................50

2-10 Geometries involved in magneto-optical Kerr imaging..........................52

2-11 Schematic of magneto-optical Kerr imaging.......................................

2-12 Cascade Microtech RF probe station............................................54

2-13 Measurement set-up with scanning electron microscopy image for domain-wall dynamic

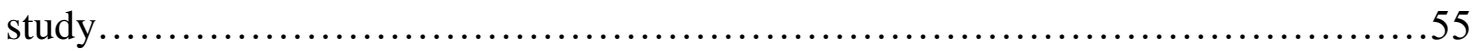

3-1 Topological defects in domain-walls and their chirality ...........................62

3-2 Simulations depicting trajectory of tail-tail-up domain-wall in branch structure..........65

3-3 Simulations depicting trajectory of tail-tail-down domain-wall in branch structure......67

3-4 Experimental results providing direct observation of chirality dependent domain-wall

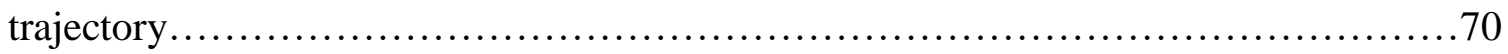

3-5 Direct observation of domain-wall evolution at the bifurcation..................... 72

3-6 Simulations depicting Walker breakdown in branch structure .......................73

3-7 Magnetic force microscopy imaging to depict stochastic domain-wall trajectory.........75

3-8 Statistical distribution of chirality dependent domain-wall trajectory $\ldots \ldots \ldots \ldots \ldots \ldots \ldots . \ldots . \ldots . \ldots$

3-9 Simulated configuration to detect sense of rotation and chirality of injected vortex domain-wall..................................................................... 77

3-10 Trajectory of vortex domain-wall in asymmetric pull-down branch structure..........78

3-11 Direct experimental observation of vortex domain-wall trajectory in asymmetric pull-

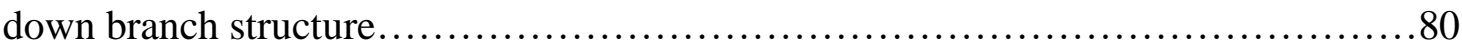

3-12 Trajectory of vortex domain-wall in asymmetric pull-up branch structure $\ldots \ldots \ldots \ldots \ldots 82$ 
3-13 Direct experimental observation of vortex domain-wall trajectory in asymmetric pullup branch structure

3-14 Simulations depicting evolution of vortex domain-wall in asymmetric structure and corresponding MFM simulations

3-15 Plot of Zeeman energy barrier for domain-wall propagation in asymmetric structure...88

3-16 Simulations depicting domain-wall propagation depending on initial vortex core polarity .90

4-1 Schematic illustrating programmable logic device with SEM image...................96

4-2 MFM image depicting zero gate current domain-wall trajectory in the logic device.......98

4-3 Simulation and MFM image depicting vortex to transverse domain-wall transformation 100

4-4 Simulation of field distribution due to current flow in the magnetic gate. 101

4-5 Experimental results depicting NAND, AND gate operations in the logic device.... 104

4-6 Experimental results depicting NOR, OR gate operations in the logic device 108

4-7 Experimental results depicting electrical detection of domain-wall using anisotropic magneto-resistance

4-8 Electrical results depicting NOR, OR gate operations in the logic device.

5-1 Out-of-plane and in-plane $\mathrm{M}-\mathrm{H}$ loops for $\mathrm{Co} / \mathrm{Ni}$ multilayer stacks with different thicknesses.

5-2 Plot of areal magnetization vs thickness in $\mathrm{Co} / \mathrm{Ni}$ multilayers

5-3 Plot of effective anisotropy per unit area vs $\mathrm{Ni}$ and Co thicknesses in $\mathrm{Co} / \mathrm{Ni}$ multilayer

5-4 SEM image and electrical measurement set-up for domain-wall dynamics....

5-5 Spin-transfer torque induced domain-wall motion in the $\mathrm{Co} / \mathrm{Ni} \mathrm{PMA}$ nanowire. 
5-6 Anomalous Hall effect (AHE) measurements and in-plane current induced domain-wall nucleation. 126

5-7 Kerr imaging of device to show multiple domain-wall nucleations

5-8 Statistical distribution of Hall resistance with current density

5-9 AHE measurements and in-plane current induced domain-wall nucleation with applied pulse widths

5-10 AHE measurements on device with larger perpendicular anisotropy

5-11 Kerr imaging of device without Hall cross after in-plane current injection

5-12 Random number generator device output with integrated electronics....

5-13 Schematic illustrating proposed system level integration of random number generator

6-1 M-H loops of $\mathrm{Pt} / \mathrm{Co} / \mathrm{Ta}$ and $\mathrm{Ta} / \mathrm{Pt} / \mathrm{Co} / \mathrm{Pt} / \mathrm{Co} / \mathrm{Ta}(\mathrm{Co} / \mathrm{Pt}$ double stack) before and after annealing..... .146

6-2 Schematic illustrating spin distribution in $\mathrm{Co} / \mathrm{Pt}$ double stack and electrical set-up for harmonic Hall measurements. 148

6-3 Magnetization dependence on external field direction and current flow

6-4 Harmonic measurements with Ta cap $=1 \mathrm{~nm}$ and Pt spacer $=0.5 \mathrm{~nm}$.

6-5 Harmonic measurements with Ta cap $=3 \mathrm{~nm}$ and Pt spacer $=0.5 \mathrm{~nm}$.

6-6 Harmonic measurements with Ta cap $=1 \mathrm{~nm}$ and Pt spacer $=1 \mathrm{~nm}$

6-7 Switching field efficiency of Co/Pt double stack with Ta and Pt thickness variation....157

6-8 Domain-wall velocity measurement on Co/Pt double stack for Ta cap $=1 \mathrm{~nm}, 3 \mathrm{~nm}$

6-9 Kerr imaging to show domain-wall motion along current flow direction using spin-orbit torque (SOT) technique. 162

6-10 Domain-wall velocity measurement on Co/Pt double stack for Pt spacer $=0.5 \mathrm{~nm}, 1 \mathrm{~nm}$ 
6-11 Kerr imaging to estimate Dzyloshinskii-Moriya interaction (DMI) field for Pt spacer = $0.5 \mathrm{~nm}$ 166

6-12 Minimum displacement plot to estimate DMI field for Pt spacer $=0.5 \mathrm{~nm}$. 167

6-13 Kerr imaging to estimate DMI field for Pt spacer $=1 \mathrm{~nm}$ 168

6-14 Minimum displacement plot to estimate DMI field for Pt spacer $=1 \mathrm{~nm}$ 169

7-1 Schematic to illustrate 3-D logic device with shared magnetic gate to perform all universal operations in one run. .179

7-2 Schematic to illustrate sloped electric field for voltage controlled domain-wall propagation. 


\section{List of Tables}

Table 1-1 Comparison of device parameters for CMOS and spintronic based logic schemes .28

Table 4-1 NAND and AND logic operations......................................106

Table 4-2 NOT and COPY logic operations......................................... 106

Table 4-3 NOR and OR logic operations..............................................

Table 5-1 Magnetic properties of $\mathrm{Co} / \mathrm{Ni}$ multilayer with respect to magnetic layer thicknesses 118

Table 5-2 Total effective anisotropy per unit area with different Co thicknesses keeping Ni thickness fixed......

Table 5-3 Total effective anisotropy per unit area with different Ni thicknesses keeping Co thickness fixed. 120 


\section{Summary}

The ever increasing digital data require memory and logic devices that have high speed, need low power, offer low cost and are reliable. Domain wall (DW) based memory and logic devices potentially offers all the above advantages along with being non-volatile. In this thesis, the dynamics of DWs have been investigated in ferromagnetic nanostructures for realizing efficient logic and memory based applications. The study has been divided into two parts. In part one, the DW dynamics in ferromagnetic network structures with in-plane magnetic anisotropy (IMA) is studied. The magnetic material chosen is NiFe due to its large magnetic permeability and near zero crystalline anisotropy. In part two of the study, materials with perpendicular magnetic anisotropy (PMA) are considered. Here, $\mathrm{Co} / \mathrm{Ni}$ and $\mathrm{Co} / \mathrm{Pt}$ multilayers are chosen as the system for study.

Field induced domain wall driving in IMA network structures revealed the DW trajectory to be chirality dependent. Deterministic trajectory of DW in the network structure is demonstrated for path lengths less than the fidelity length and for fields less than Walker breakdown field. For distances in excess of fidelity length, Walker breakdown is observed where DW chirality is found to oscillate and the trajectory becomes stochastic. To overcome this issue, the network structures are re-designed to include geometrical asymmetry. This constrains DW to propagate in a definite and fixed branch, by raising the potential barrier in the other branch, irrespective of its chirality. This branch engineering is applied to propose and demonstrate a programmable logic device. The control of the trajectory is provided by fabricating a metallic gate on top of the network structure. The current in the gate applies an Oersted field which influences the trajectory of DW by interacting with the transverse charge distribution in the DW. A transverse nanowire in the structure serves a dual purpose of acting as an input logic and also transforming the vortex DW to a transverse chirality. Two universal 
logic gate functionalities NAND and NOR are demonstrated along with the complimentary logic operations in the same structure using magnetic force microscopy imaging and anisotropic magneto-resistance.

The PMA system is investigated using the phenomena of anomalous Hall effect. Our investigations reveal multiple DW nucleations in low anisotropy $\mathrm{Co} / \mathrm{Ni}$ Hall cross junctions under the application of in-plane current without the need of conventionally used local Oersted field. The stochasticity in the nucleation process due to high demagnetization energy at the Hall cross coupled with joule heating is utilized to demonstrate a DW based random number generator device. The analog signal from the device is fed to an integrated circuit to amplify and digitize the output. Recent developments have shown combination of interfacial phenomenon such as spin-orbit torque (SOT) and Dzyloshinskii-Morya interaction leading to high speed DW dynamics. Pt/Co/Ta stacks have enhanced SOT strength due to opposite signs of spin Hall angle of Ta and Pt. However, it is observed that stack possesses low thermal stability due to reported intermixing of Ta and Co. A solution to this issue is provided by adding additional $\mathrm{Co} / \mathrm{Pt}$ interface which enhances the thermal stability and PMA strength. The distribution in velocity with the applied current density and spin Hall angle is estimated by changing the Ta capping thickness and Pt spacer thickness. SOT driven DW speed of about $530 \mathrm{~m} / \mathrm{s}$ at a current density of $1 \times 10^{12} \mathrm{~A} / \mathrm{m}^{2}$ is demonstrated. This high speed is attributed to low anisotropy of our device coupled with in-plane field applied to prevent Walker breakdown. DW velocity was higher and along the current flow direction when the in-plane field assisted the inherent DMI field, while it was lower and along the electron flow direction when the field opposed the DMI field. 


\section{Chapter 1}

\section{Introduction}

Silicon based transistors have dominated the micro-electronics industry for decades. Moore's law, which states that number of transistors would double every eighteen months, has been the guiding principle for this technological revolution [1]. However, the limit of Moore's law is fast approaching. The major issue of scaling down is heat generation. To avoid heating issues in chips, the clock rates have been limited and have not changed since 2004 [2, 3]. By 2020 the expected limit of device dimensions is $2-3 \mathrm{~nm}$ which is 10 atoms across. At such small scales the quantum effects would start to dominate, making transistor operations extremely unreliable. Spintronics which combines charge of an electron with spin degree of freedom offers an alternate to the silicon technology. The advent of spin-transfer torque (STT) $[4,5]$ and more recently spin-orbit torque (SOT) [6-8] techniques have revolutionized the way in which data or spin can be manipulated by using electric current. Amongst the key emerging memory technologies, spin-transfer torque magnetic random access memory (STT-MRAM) is the only non-volatile memory expected to have infinite endurance [9]. This property of endurance along with the ability to scale down below $20 \mathrm{~nm}$ makes STT-MRAM a promising candidate to replace the volatile DRAM [10]. With further improvements in reading and writing, STTMRAM could replace embedded DRAM (eDRAM) as a cache memory application.

Even though there is no inherent magnetic wear-out in STT-MRAM, there is however an electrical wear-out which is the dielectric breakdown of the $\mathrm{MgO}$ tunnel barrier. To avoid this issue, the write voltage must be kept low, below $400 \mathrm{mV}$ [11]. Another solution for this is SOTbased MRAM [12,13], which is a three terminal device and separates the read and write path for the current. However, SOT-MRAM poses scaling issue to low dimensions. Moreover, the fabrication and process steps in MRAM are complex. A possible memory alternative which 
combines the positive attributes of MRAM along with having simplified fabrication steps and avoiding the usage of tunnel barrier is domain wall (DW) based racetrack memory (RM) [1417]. RM stores bits of data as magnetized regions in a nanowire. RM can also offer as a higher speed and reliable alternative of hard-disk drives (HDD) since it does not have moving parts. The operation of the RM is analogous to a non-volatile shift register. On one end the bits may be written by changing the magnetization directions in the nanowire. The bits can be pushed by using STT and read using a magnetic tunnel junction or Hall probe as the sensor. The RM offers a possibility to re-orient the structure in the vertical direction and store bits in a threedimensional geometry, thereby increasing the storage density. However, exploratory prototypes exist in two-dimensions. DW dynamics can also be employed in designing magnetic logic devices. Allwood et al. proposed the first DW based logic device [18, 19], utilizing the geometry of the pattered nanostructures and a rotating magnetic field. Recently, we proposed a reconfigurable DW logic device exploiting the structural asymmetry and a current controlled magnetic gate [20].

Current research interest lies in high speed DW dynamics and reduction of the current density for DW propagation. Research in this field has gained momentum following recent developments in employing anti-symmetric exchange interaction, namely DzyaloshinskiiMoriya interaction (DMI) [21, 22], assisted SOT techniques for DW driving [23-27]. However, studies thus far have focussed on single ferromagnetic layer to obtained enhanced SOT. This poses a scaling issue due to lower thermal stability. There is a need to explore DW dynamics in alternate multilayer structures which have higher perpendicular magnetic anisotropy (PMA) and thermal stability. In this thesis, we have explored DW dynamics in in-plane magnetic anisotropy (IMA) as well as PMA based structures. In this chapter we would focus on the basic theory behind ferromagnetism and domain wall dynamics as well as review some of the recent literature. 


\subsection{Basic Theory of Ferromagnetism and Domain formation}

In the absence of external magnetic field, the spins of most materials orient in random directions and the net magnetic moment is zero. The class of such materials fall under either diamagnetic which are repelled by magnetic field and paramagnetic materials which are attracted by magnetic field. A class of materials having spontaneous magnetization without any external field are called ferromagnetic. The phenomena of ferromagnetism can be explained from quantum mechanics and it is difficult to describe using a classical picture. In terms of quantum phase transitions, the breaking of spin rotational symmetry leads to a phase transition from paramagnetic to ferromagnetic below the Curie temperature [28, 29].

It was observed in some materials that the saturation magnetization could be reached with the application of a very small magnetic field and the magnetization could be zero at zero applied field. This led Weiss to propose an internal molecular field responsible for the attainment of large magnetization and existence of domains to reduce the net magnetization to zero. The molecular field required to overcome the thermal agitation at Curie temperature $\mathrm{kT}_{\mathrm{c}}$ is approximately $\mathrm{kT}_{\mathrm{c}} / \mu_{\mathrm{B}}$ and has a value of $10^{7}$ Oersted [30]. This is larger than any field present on lab level. The magnetic moment of ferromagnetic materials arises primarily from spin angular momentum and orbital angular momentum provides roughly $10 \%$ contribution [31, 32]. Also the electrons responsible for ferromagnetism contribute very little to the conduction since ferromagnetism is generally associated with $\mathrm{d}$ and $\mathrm{f}$ orbitals which are localized, whereas the s orbital electrons are delocalized and contribute to the conduction.

The origin of the Weiss molecular field is exchange interaction and follows directly from Pauli's exclusion principle that no two electrons can have all the same quantum numbers, in conjunction with Coulomb repulsion between two electrons on neighbouring atoms. If the two electrons are indistinguishable, the density functions after exchanging the two electrons must be equal i.e. $|\Psi(1,2)|^{2}=|\Psi(2,1)|^{2}$, the total wave function is antisymmetric since electrons are 
fermions i.e. $\Psi(1,2)=-\Psi(2,1)$. The total wave function is a product of spatial and spin coordinates $\Phi\left(\mathrm{r}_{1}, \mathrm{r}_{2}\right)$ and $\chi\left(\mathrm{r}_{1}, \mathrm{r}_{2}\right)$, respectively. These coordinates have their respective symmetric and anti-symmetric components which for spin are referred to as triplet and singlet, respectively. The total wave function should be a product of symmetric part of spatial coordinate with anti-symmetric part of spin (singlet spin) coordinate and vice versa. Thus we have [33]:

$$
\begin{aligned}
& \Psi_{I}=\Phi_{s}(1,2) \chi_{a}(1,2) \\
& \Psi_{I I}=\Phi_{a}(1,2) \chi_{s}(1,2)
\end{aligned}
$$

Thus electrons which are in spin triplet state cannot be found at the same point in space. The energy splitting between singlet and triplet state is $2 \mathrm{~J}$, where $\mathrm{J}$ is the exchange integral,

$$
\mathrm{J}=\int \Psi_{1}^{*}\left(r^{\prime}\right) \Psi_{2}^{*}(r) H\left(r, r^{\prime}\right) \Psi_{1}(r) \Psi_{2}\left(r^{\prime}\right) d r^{3} d r^{\prime 3}
$$

The Hamiltonian, generalized for all pair of lattice sites $i, j$ is expressed as [33]:

$$
H=-2 \sum_{i, j} J_{i j} S_{i} \cdot S_{j}
$$

$J$ is positive if the interaction is ferromagnetic and negative if the interaction is antiferromagnetic. The exchange energy is more generally represented in terms of exchange stiffness constant, A and the misalignment angle between the spins, $\theta$, separated by distance $\mathrm{x}$.

$$
E_{e x}=A\left(\frac{\partial \theta}{\partial x}\right)^{2}
$$

where, $A=2 n J S^{2} / a, a$ being the lattice constant and $n$ is the number of atoms per unit cell.

In general, the magnetization in a ferromagnetic material lies along a preferred crystallographic direction known as the easy axis of magnetization. This intrinsic property is referred to as magneto-crystalline anisotropy $\mathrm{E}[30]_{\mathrm{a}}$ :

$$
E_{a}=K \sin ^{2} \theta
$$


where, $K$ is the anisotropy constant and $\theta$ is the angle between the magnetization direction and the easy axis. The origin of magnetic anisotropy is the crystal field interaction, spin-orbit coupling and dipole-dipole interaction. The potential due to the crystal field interacts electrostatically with the orbitals containing the magnetic electrons. This interaction tends to stabilize a particular orbital and through spin-orbit interaction, the magnetic moment is oriented along a particular crystallographic direction. E.g. in cobalt, the hexagonal axis is the easy axis, in iron which is cubic, the cube edges [100] are preferred directions and in Ni the body diagonal [111] is the easy axis of magnetization [30].

From Maxwell's relation, $\nabla \cdot \boldsymbol{B}=0$ and $\nabla \cdot \boldsymbol{H}=-\nabla \cdot \boldsymbol{M}$ which implies the existence of flux closure, preventing free poles from existing. The magnetic poles generate a demagnetizing or stray field $\boldsymbol{H}_{\boldsymbol{d}}$, raising the energy of the material in the form of magnetostatic energy. This energy is also termed as shape anisotropy, since it depends on the shape of the magnetic material and resists the orientation of moments along a particular direction. For instance, in a needle shaped magnet the moments would tend to align along the long axis since it would minimize the free poles, since if the moments were orthogonal to the needle axis the separation between the free poles would reduce increasing the magnetostatic energy [30].

$$
E_{m s}=\frac{1}{2} \mu_{0} N M_{S}^{2}
$$

where, $N$ is the demagnetizing factor, generally represented as a tensor with unit trace. $\mathrm{N}$ depends on geometry, for instance, $N=1 / 3$ for a sphere, $N=1 / 2$ and 0 for a needle with magnetization pointing along perpendicular direction and along the needle axis, respectively. For a thin film, $N=0$ and 1 for magnetization along the film plane and perpendicular to it, respectively $[34,35]$. 

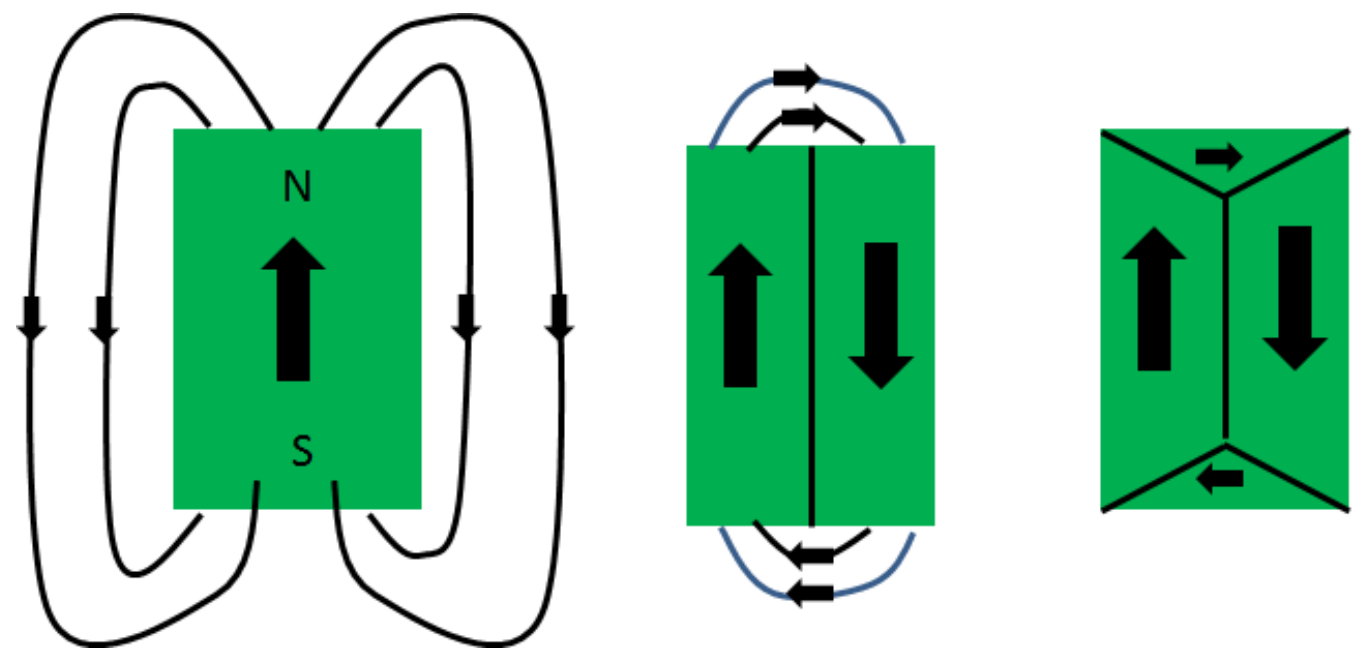

Figure 1-1 Schematic illustrating formation of domains in a ferromagnetic material to reduce the stray field and lower the demagnetization energy. Adapted from [30].

(a)

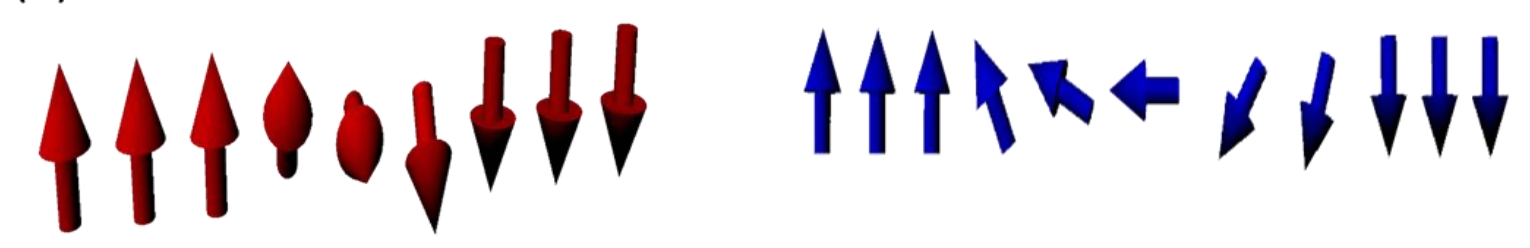

Figure 1-2 Types of domain walls (DW) (a) Bloch wall (b) Néel wall. Adapted from [33]

\subsection{Domain Wall}

Every ferromagnetic system, in the absence of external perturbation, strives to attain minimum energy state i.e. the sum total of magnetostatic, anisotropy and exchange energy should be minimum. The minimization of magnetostatic energy divides the magnetization configuration into domains as shown in Figure 1-1. Each domain has spins or moments point along a preferred direction by simultaneous minimization of magneto-crystalline and shape anisotropy energy. The transition from one domain to the next is gradual due to the minimization of exchange energy and this separation between domains is called a domain wall (DW). The sense 
of rotation of the spins within a DW gives rise to two configurations - Bloch wall and Néel wall as shown in Figure 1-2. The width of domain wall is finite due to two competing energy terms, exchange specified by stiffness parameter A and anisotropy specified by anisotropy constant K. In cases where exchange dominates, the wall width is large and in cases where anisotropy dominates, the wall width is narrow [30].

$$
w=\sqrt{\frac{A}{K}}
$$

The wall formation also costs energy, since moments inside a domain may not align perfectly parallel to each other costing exchange energy and may deviate away from the easy axis costing anisotropy energy. The energy per unit area of a Bloch wall is [33]

$$
\sigma_{B W}=\pi \sqrt{A K}
$$

Néel wall, where the magnetization rotates in the plane of the domains, is higher in energy than the Bloch wall due to the stray field which creates non-zero divergence of M. Néel walls are stable if the film thickness is less than the wall thickness. Figure 1-3 shows a schematic representing Néel wall by elliptic cylinder of cross-section $t \times \delta$, where $t$ is the film thickness and $\delta$ is the wall width. For bulk sample, the demagnetizing factor for the Bloch wall is zero and for the Néel wall is 1 . However, when t is reduced and becomes comparable to $\delta$, the Néel wall has lower magnetostatic energy.

The coercivity of a homogeneous and uniformly magnetized ellipsoid obeys the relation, $H_{c}$ $\geq 2 K_{1} / \mu_{0} M_{s}-N M_{s}$ [33]. This is known as Brown's theorem. In practice the coercivity is much smaller than that predicted by Brown's theorem. This is because, real materials are inhomogeneous and magnetization reversal is initiated in a small nucleation volume around a defect site. Local stray fields around a defect side can lead to magnetization reversal. The DW, thus formed may expand on application of external magnetic field or stay pinned around the 


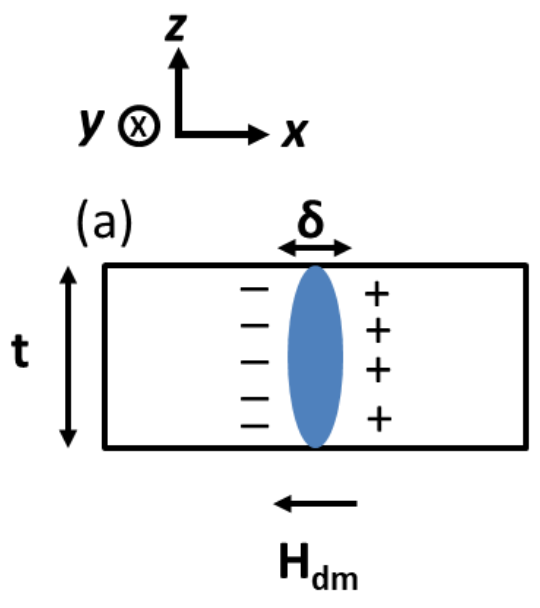

$t>\delta$ (b)

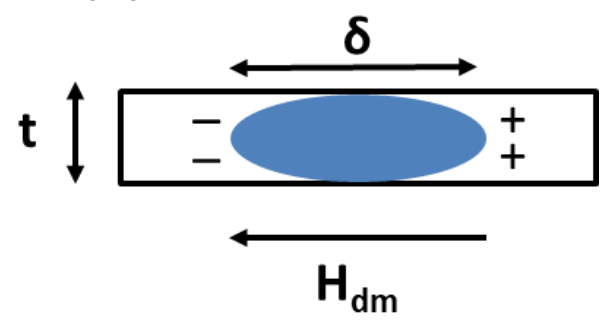

$\mathbf{t}<<\delta$

Figure 1-3 Schematic to illustrate strength of demagnetizing field in $180^{\circ}$ domain wall (a) Thick film has larger demagnetization field along y-direction than (b) Thin film. Adapted from [35] .

defect in the absence of external field. If the particle size is very small, it would prefer to be in a single domain state since the energy gained by the wall formation is not compensated by any reduction in magnetostatic energy. If we consider a spherical particle of radius $\mathrm{R}$, then the critical maximum size of single domain particle, $\mathbf{R}_{\mathrm{sd}}$ is given by [33],

$$
R_{s d}=9 \sqrt{A K} / \mu_{0} M_{s}^{2}
$$

In general, two different rotation mechanisms of single domain particles are possible. First is the coherent rotation of spins in unison proposed in the Stoner-Wohlfarth model [36]. This creates stray field energy as the spins flip. The second is the curling mode, where the spins form a vortex state avoiding stray field since the magnetization is always parallel to the surface, however, the exchange energy increases. The vortex state is common in soft magnetic particles which are larger than the coherence length [33]. 
(a)

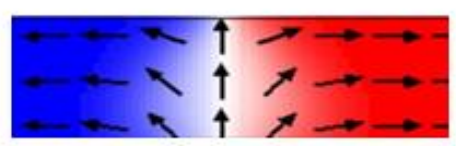

(b)

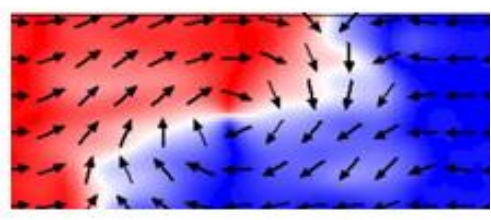

(c)

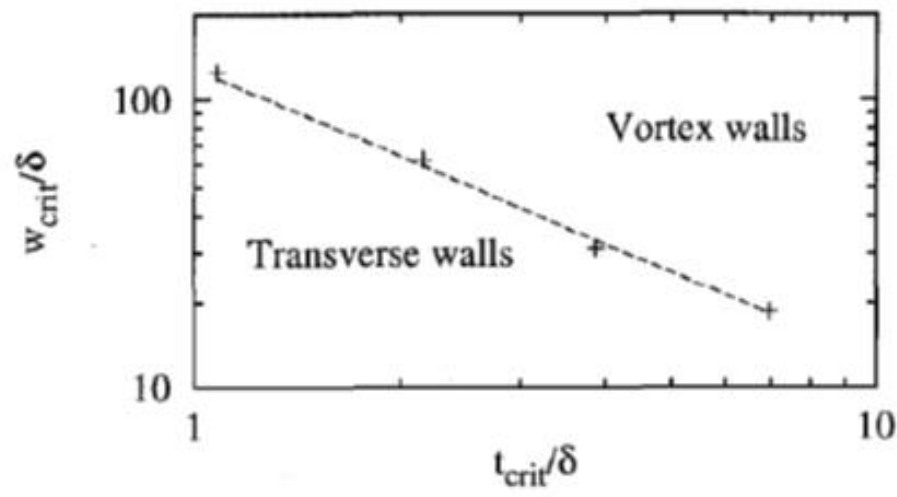

Figure 1-4 Micromagnetic (OOMMF) simulations to illustrate (a) Transverse DWs and (b) Vortex DWs (c) Phase diagram depicting transition from transverse to vortex as width and thickness increase, adapted from [37].

The configuration or chirality of DW is determined from the minimization of all the energy terms (magnetostatic, exchange and anisotropy). In IMA thin nanostrips, specifically permalloy $\left(\mathrm{Ni}_{80} \mathrm{Fe}_{20}\right)$, studied in this thesis, Néel walls are formed due to near zero crystalline anisotropy and the domains lie along the long-axis due to shape anisotropy. Thin, narrow strips exhibit transverse DWs as depicted in Figure 1-4 (a). Thick narrow strips prefer vortex DWs as shown in Figure 1-4 (b). Figure 1-4 (c) shows the phase diagram, taken from reference [37], depicting the transition between different wall types depending on the wire dimensions. The width of the DW, in IMA nanostrips is of the order of strip width. In nanostrips with PMA, the configuration of DW is either Bloch, where the spins rotate out of the plane of magnetization, or Néel, where spins rotate in the plane of magnetization as was shown in Figure 1-2. Koyama et al. [38] demonstrated the transition from Bloch to Néel wall occurs for nanowire widths less than $60 \mathrm{~nm}$. 


\subsection{Domain Wall Dynamics}

DWs can be made to propagate under the influence of external magnetic field to minimize Zeeman energy and under the influence of current due to angular momentum transfer from electrons in the current to the moments of the DW. Two torque transfer mechanisms have been proposed in literature namely, spin-transfer torque (STT) in conventional ferromagnets and spin-orbit torque (SOT) present in systems with heavy-metal and ferromagnetic interface. The dynamics are governed by the Landau-Lifshitz-Gilbert (LLG) equation [39] which is discussed in the following sections.

\subsubsection{Field-driven Domain Wall Dynamics}

Magnetization dynamics can be modelled using LLG equation, which relates the rate of change of magnetization to the different torque contributions.

$$
\frac{\partial \boldsymbol{m}}{\partial t}=-|\gamma| \boldsymbol{m} \times \boldsymbol{H}_{e f f}+\alpha \boldsymbol{m} \times \frac{\partial \boldsymbol{m}}{\partial t}
$$

where, $\alpha$ is the Gilbert damping parameter [40]. The first term on the right hand side represents the precession of the magnetic moment around the effective magnetic field. The effective magnetic field would have contribution from exchange, anisotropy, magnetostatics as well as external Zeeman field. The second term represents the damping of the magnetic moment towards the effective field. Figure 1-5 shows the schematic representing precession and damping.

Figure 1-6 shows the field driven DW dynamics in IMA nanostrip with transverse DW. The torque $-|\gamma| \boldsymbol{m} \times \boldsymbol{H}_{\text {eff }}$ acting on the DW moment, lifts the moment out of the plane, which in turn generates a demagnetizing field, $\boldsymbol{H}_{\boldsymbol{d}}$, opposing the moment. At low enough applied field when the canting angle is small the DW is driven forward by the torque due to $\boldsymbol{H}_{\boldsymbol{d}}$, which is $-|\gamma| \boldsymbol{m} \times \boldsymbol{H}_{\boldsymbol{d}}$. When the field becomes greater than a certain field $\boldsymbol{H}_{\boldsymbol{w}}$, known as 
(a)

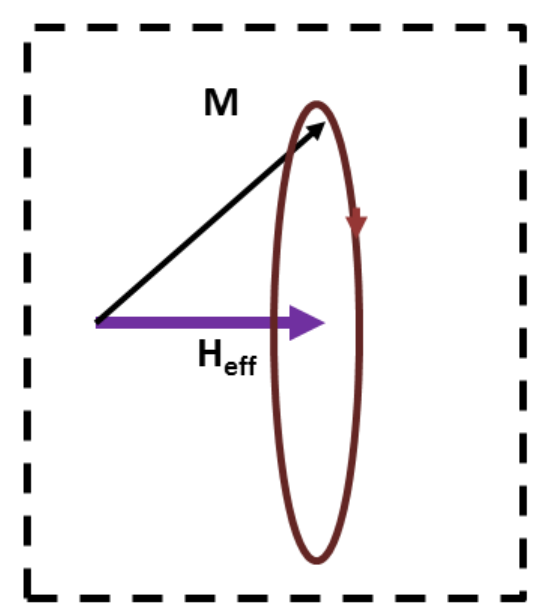

Larmor term:

precession (b)

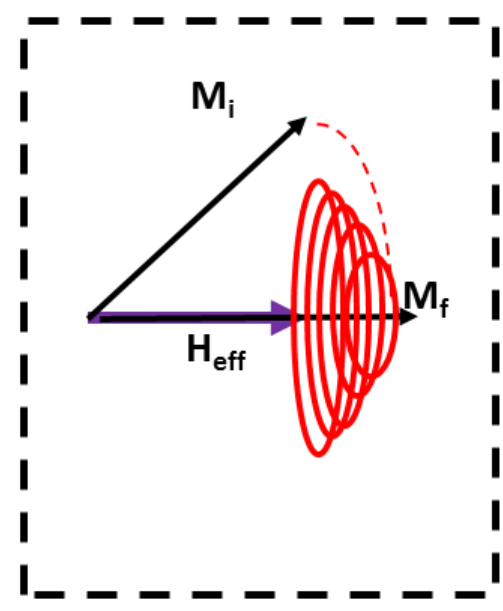

Damping term: aligns $\mathrm{M}$ with $\mathrm{H}$

Figure 1-5 Schematic to show (a) Precession of magnetization around effective magnetic field (b) Damping of magnetization towards the effective field.

Walker field [41], the DW precesses about the $x$-axis and DW is driven by the damping torque $\alpha \boldsymbol{m} \times \frac{\partial \boldsymbol{m}}{\partial t}$. The velocity of DW below the Walker field is $\mathrm{v}=\mu \mathrm{H}$, where $\mu$ is the mobility $[42,43]$. Above the Walker field the velocity tends to $\alpha^{2} \mu \mathrm{H}$. Since $\alpha<<1$, the DW mobility drops significantly above Walker field. This is termed as Walker breakdown [41]. Figure 1-7 shows the mobility curves adapted from reference [35]. Field driven DW dynamics in PMA nanostrips are depicted in Figure 1-8. Consider the case of a Bloch wall, the spins precess about the $z$-axis and tend to adopt a Néel configuration. The demagnetizing field generated along $x$-direction drives the DW when the field is less than the Walker field. As field is increased beyond the Walker field the DW is driven by the damping torque. It is worth noting that in PMA nanostrips the magnetostatic energy penalty for canting the DW moment to Néel configuration is less than the energy expended in IMA nanostrips to cant the moment out of plane. Thus the Walker field $\mathrm{H}_{\mathrm{W}}$ and hence the maximum DW 

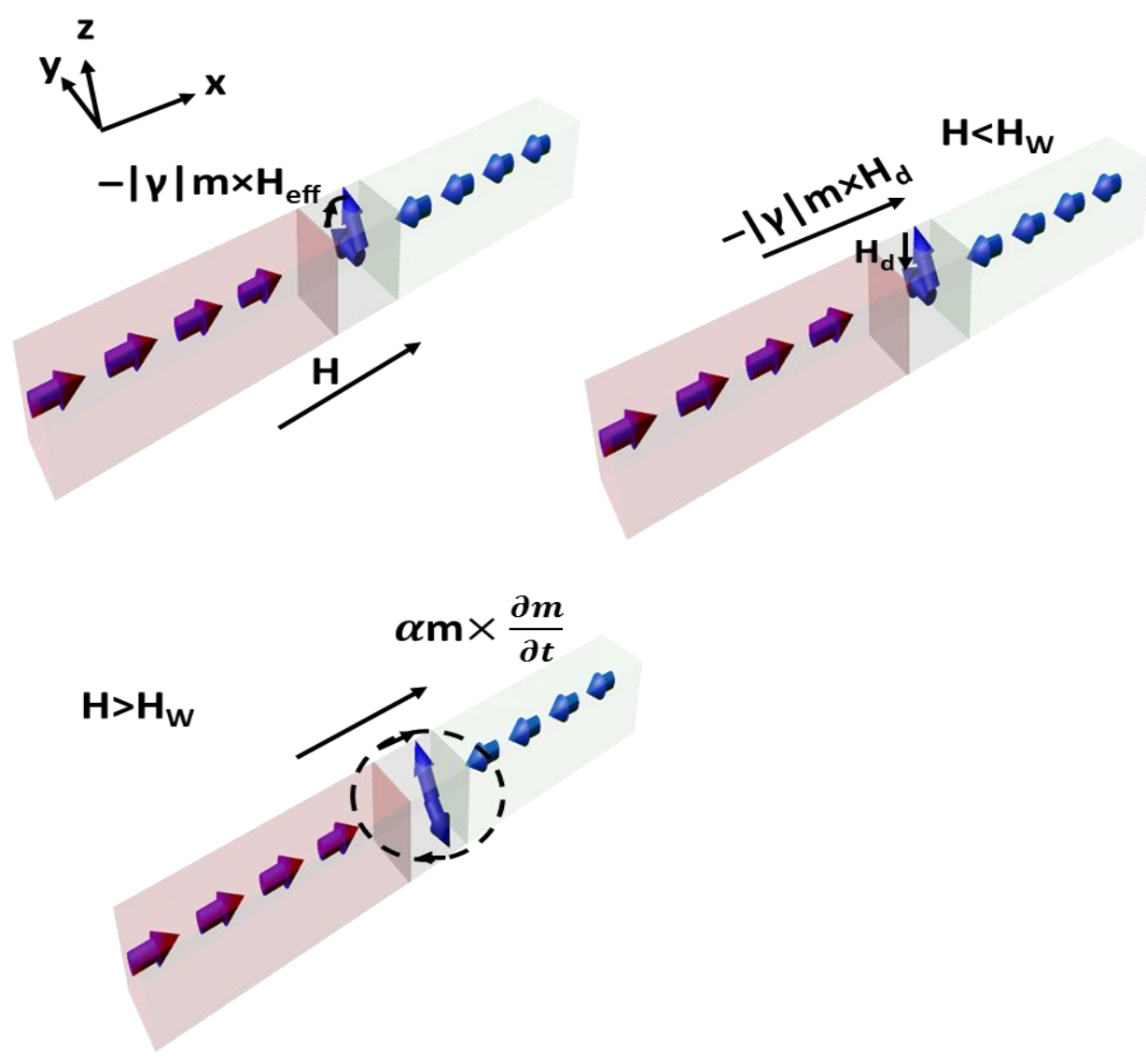

Figure 1-6 Field driven DW dynamics for in-plane magnetized nanowire. The moment precesses out-of-plane in the presence of field and creates demagnetization field to counter it. The DW is driven by the demagnetization field when applied field is less than Walker breakdown limit, beyond the Walker breakdown the damping torque drives the DW through precessional motion. Adapted from 2D images in [44]

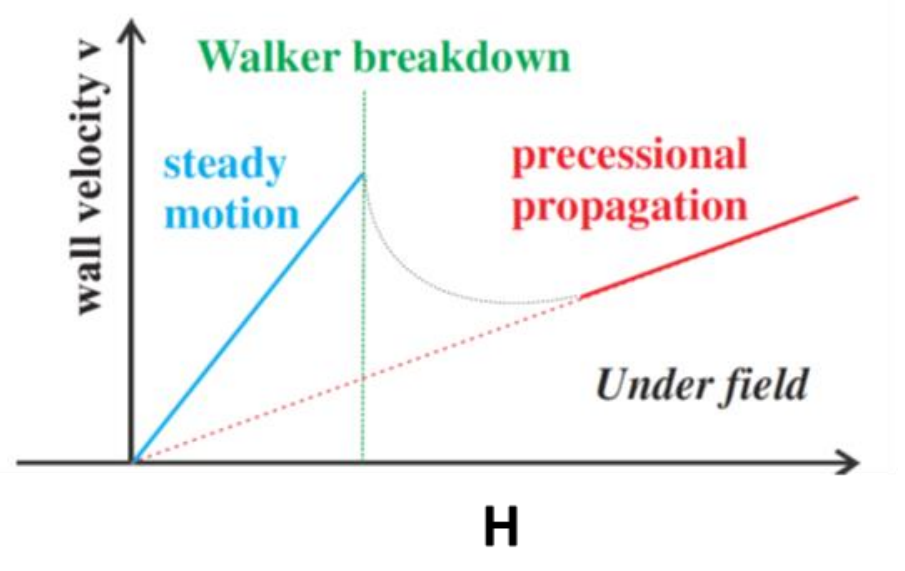

Figure 1-7 Plot of velocity with applied magnetic field illustrating steady state motion and precessional motion beyond Walker breakdown. Adapted from [35] . 
velocity is less for the case of PMA than in case of IMA nanostrips when driven by field. The lower energy barrier for precession in the case of PMA nanostrips is beneficial when driven by current.
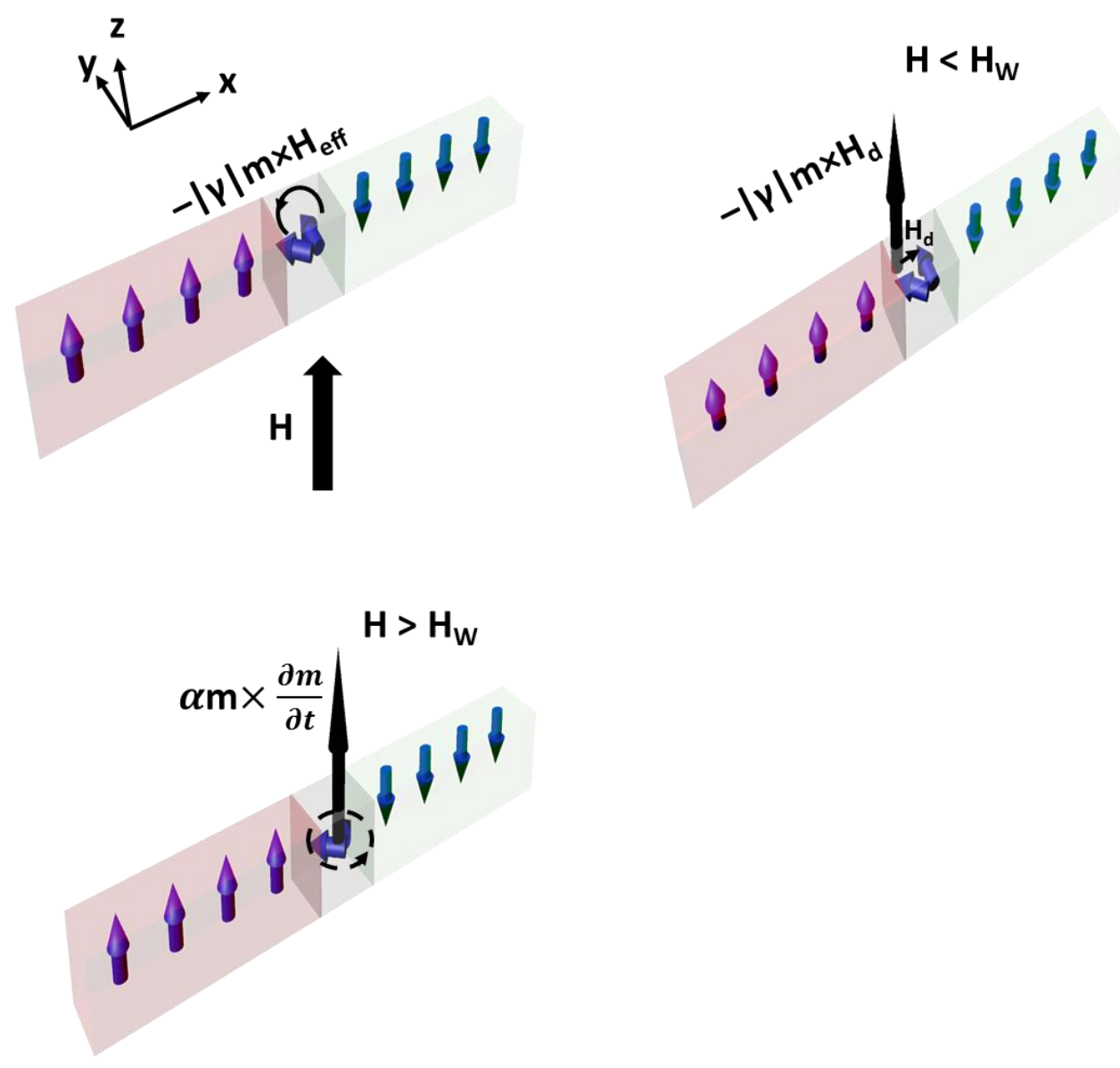

Figure 1-8 Field driven DW dynamics for out-of-plane magnetized nanowire. Dynamics can be explained similar to the in-plane magnetized case. Adapted from 2D images in [44]

\subsubsection{Current-driven Domain Wall Dynamics}

Electrons flowing through a ferromagnetic material become spin polarized i.e. majority of their spins orient in the direction of magnetization of the material. This is due to the scattering of the injected spins by the moments through s-d exchange interaction [45], making the scattering cross-section dependent on the angle between moments and the spins. Due to conservation of total angular momentum, the moments are also affected after they polarize the injected spins. This transfer is very efficient when the spins and moments are 
non-collinear as in the case of a DW. The moments in the DW are rotated towards the spin by the torque induced by the spin angular momentum and the incoming spins align along the direction of moment of the DW. Thus, this leads to DW motion and the process is called spin-transfer torque (STT) which is illustrated in Figure 1-9. STT has been classified into adiabatic and non-adiabatic components. Recently another torque transfer mechanism has been highlighted, namely spin-orbit torque (SOT) existing at the interface of heavy-metal and ferromagnetic interface. It's origin is spin-orbit coupling and crystal field in asymmetric stacks. The various mechanisms of torque transfer are discussed briefly.
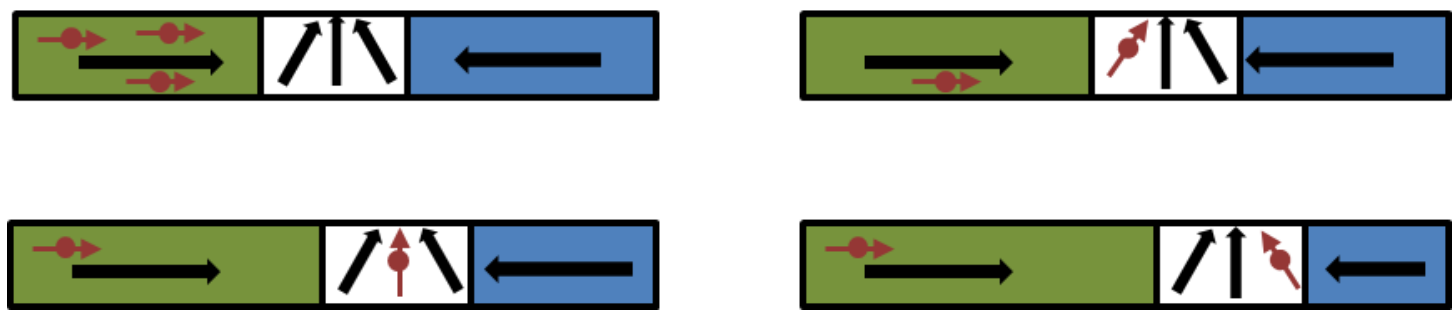

Figure 1-9 Schematic depicting transfer of angular momentum from spin polarized current to the DW moments leading to its motion via spin-transfer torque to conserve the total angular momentum. Adapted from [33]

\subsubsection{Adiabatic Spin-transfer Torque}

The LLG equation is modified after incorporating the adiabatic torque term [40]

$$
\frac{\partial \boldsymbol{m}}{\partial t}=-|\gamma| \boldsymbol{m} \times \boldsymbol{H}_{e f f}+\alpha \boldsymbol{m} \times \frac{\partial \boldsymbol{m}}{\partial t}-(\boldsymbol{u} \cdot \nabla) \boldsymbol{m}
$$

where, $\boldsymbol{u}$ is the spin drift velocity,

$$
\boldsymbol{u}=\frac{g \mu_{B} P \boldsymbol{J}}{2|e| M_{S}}
$$

$P$ is the spin polarization and $\mathrm{J}$ is the current density. The adiabatic STT acting on a DW would be proportional to the gradient of magnetization. Consider the case of perpendicularly 
magnetized nanostrip with a Bloch DW, which is the magnetostatically preferred configuration, under the application of low current. Figure 1-10 shows the DW dynamics under the application of adiabatic STT. When the current is injected along $-x$-direction, the spins polarized in the $+z$-direction exert a damping-like torque on the DW moment which tries to orient its moment along the $+z$-direction. This induces a damping torque, $\alpha \boldsymbol{m} \times \frac{\partial \boldsymbol{m}}{\partial t}$ rotating $\boldsymbol{m}$ in the clockwise direction in the plane and thus deviating away from the Bloch configuration. This initiates a demagnetizing field, $\boldsymbol{H}_{\boldsymbol{d}}$ acting along the $-x$-direction, which generates a torque $-|\gamma| \boldsymbol{m} \times \boldsymbol{H}_{\boldsymbol{d}}$ along the $-z$-direction countering the adiabatic torque due to injected spins. This torque in-turn generates a damping torque rotating magnetization along the counter-clockwise direction. Thus all the torques balance out and there is no motion of the DW. This is referred to as intrinsic pinning and there is a threshold current required to cause DW motion [38]. If the current exceeds this threshold the demagnetization field torque would not fully compensate the damping torque and DW would precess continuously and the velocity is non-uniform with periods of acceleration and de-acceleration. Similar line of explanation also holds true for DW dynamics in IMA nanostrips.

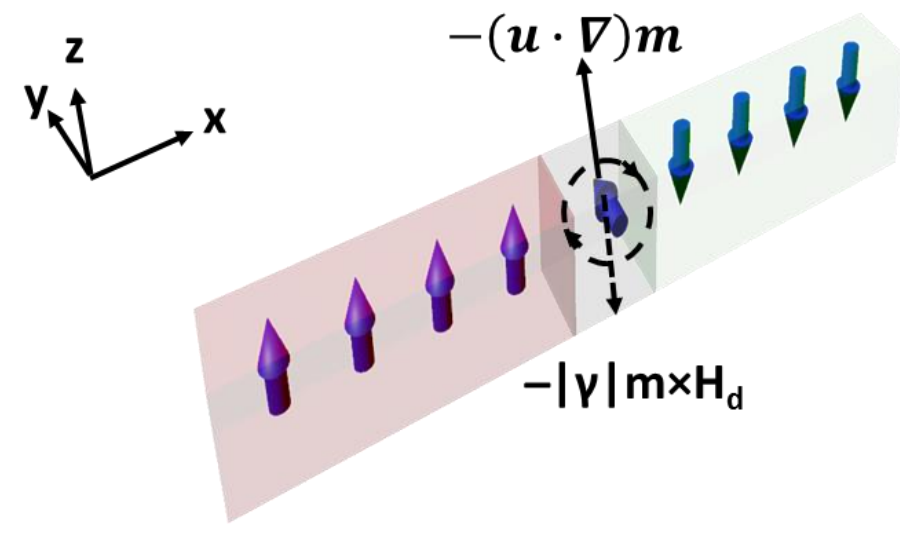

Figure 1-10 Current driven DW dynamics for out-of-plane magnetized nanostrip through adiabatic spin-transfer torque (STT). DW moves by precession about the $\mathrm{x}$-axis when the adiabatic torque can overcome the torque due to demagnetization field. Adapted from [44] 


\subsubsection{Non-adiabatic Spin-transfer Torque}

According to the theory of adiabatic STT, the current density required for DW motion is as high as $10^{13} \mathrm{~A} / \mathrm{m}^{2}$ [46]. However many experimental observations report a one order lower current density required for propagation. This has led to the proposal of additional torque mechanism namely, the non-adiabatic STT $[47,48]$. The modified LLG equation is [40]:

$$
\frac{\partial \boldsymbol{m}}{\partial t}=-|\gamma| \boldsymbol{m} \times \boldsymbol{H}_{e f f}+\alpha \boldsymbol{m} \times \frac{\partial \boldsymbol{m}}{\partial t}-(\boldsymbol{u} \cdot \nabla) \boldsymbol{m}-\beta \boldsymbol{m} \times[-(\boldsymbol{u} \cdot \nabla) \boldsymbol{m}]
$$

The non-adiabatic STT term is orthogonal to the adiabatic STT term and is equivalent to a torque produced due to a magnetic field [40]. This torque term makes the pinning extrinsic instead of the intrinsic pinning and can lead to larger DW speeds without precessions. Since the symmetry of the non-adiabatic torque is that of a field, it is also called field-like STT. The presence of non-adiabatic component along with adiabatic STT makes the DW dynamics to depend on the ratio of $\beta$ to $\alpha$. Figure 1-11 shows the DW dynamics under the application of non-adiabatic torque. When $\beta<\alpha$ and the current density is small, the fieldlike torque would be able to compensate the damping torque due to adiabatic STT and the demagnetizing field. The damping torque due to the non-adiabatic STT would compensate the torque due to non-adiabatic STT. Thus the contribution for DW motion would come from the torque due to demagnetizing field which would drive the DW forward without precession. On further increasing the current, the damping torque due to adiabatic STT is able to compensate the damping torque due to the demagnetizing field and the field-like torque i.e. the torque due to non-adiabatic STT. Thus the DW precesses, however the velocity still increases since the precession generates a torque in the same direction as that due to demagnetizing field. When $\beta>\alpha$, the field-like torque becomes larger than damping torque due to adiabatic STT and the demagnetizing field. This causes the DW to precess continuously and generate a torque to partially compensate the torque due to demagnetizing field. Thus there is a decrease in DW velocity due to the precession. This corresponds to 
Walker breakdown and the DW velocity decreases with increase in the current. The velocity of DW below the Walker breakdown is $\mathbf{v}=\beta \mathbf{u} / \alpha$ and approaches $\mathbf{u}$, when current increases beyond the Walker breakdown limit [40].

The physical origin of non-adiabatic STT may be due to spin mistracking, i.e. when conduction electrons may get scattered from narrow DWs instead of tracking the moments and directly transfer linear momentum [46]. The ratio of non-adiabaticity parameter, $\beta$, to the Gilbert damping parameter, $\alpha$, is crucial in ascertaining the DW dynamics. Some studies propose $\beta / \alpha \sim 1[49,50]$, while more recent studies propose $\beta / \alpha \sim 10[51,52]$.

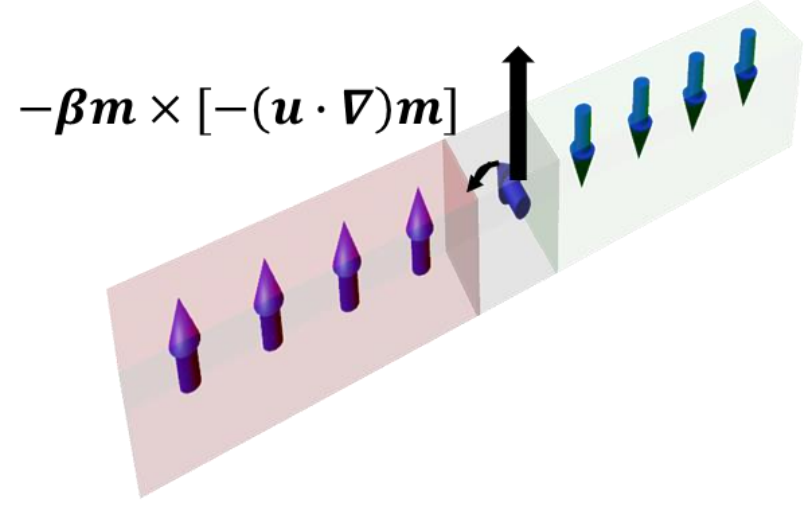

Figure 1-11 Current driven DW dynamics for out-of-plane magnetized nanostrip through nonadiabatic STT. Non-adiabatic torque is orthogonal to adiabatic STT. DW is driven by translational motion rather than precession. Adapted from [44]

\subsubsection{Spin-orbit torque}

Many interesting phenomena observed in ferromagnetic materials are attributed to the existence of spin-orbit interaction. An electron possesses orbital as well as spin angular momentum. These may be coupled to give rise to total angular momentum $\mathrm{j}$, such that $\mathrm{m}=$ $\gamma \mathrm{j}$ where $\gamma$ is the gyromagnetic ratio. The basic principle is that in the rest frame of an electron, the nucleus revolves around it with speed $\mathrm{v}$, generating a current loop $I=Z e v / 2 \pi r$, 
where $\mathrm{Z}$ is the atomic number. This current produces a magnetic field $\mu_{0} \mathrm{I} / 2 \mathrm{r}$ at the centre. This field is responsible for the spin-orbit coupling, $\boldsymbol{B}_{\boldsymbol{s}}=\mu_{0} \mathrm{Zev} / 4 \pi r^{2}$. The interaction energy $E_{s o}$ can be expressed in terms of Bohr magneton and Bohr radius [33]:

$$
E_{s o} \approx-\frac{\mu_{0} \mu_{B}^{2} Z^{4}}{4 \pi a_{0}^{3}}
$$

The spin-orbit interaction becomes strong for heavy elements due to $Z$ dependence and especially for inner shells. This is one primary reason for interfacial effects observed when heavy metals like Pt, Ta, W are used as underlayer or capping layer of a thin ferromagnetic film.

In a non-magnetic material, if unpolarised electrons are injected along $+x$-direction, a spin current would be generated along $y$ and $z$-directions which have spin polarizations along $z$ and $y$-directions respectively. This is spin Hall effect (SHE) due to spin-orbit coupling [53]. There is no net charge accumulation since there is no majority spin polarization. If the material is magnetized along $z$-direction, there is preferential polarization of electron along the $z$-direction, thus on account of spin dependent scattering there is charge accumulation along the $y$-direction. This broken time reversal symmetry corresponds to the anomalous Hall effect (AHE) [54]. In systems with broken inversion symmetry having a ferromagnet and heavy metal interface e.g. $\mathrm{Pt} / \mathrm{Co} / \mathrm{AlO}_{\mathrm{x}}$ or $\mathrm{Ta} / \mathrm{CoFeB} / \mathrm{MgO}$, there exists another source of SOT, namely, Rashba effect $[55,56]$. These materials exhibit asymmetric crystal field in the $z$-direction (out-of-plane). From the rest frame of electrons moving in the ferromagnet, the electric field is an equivalent magnetic field, which polarizes electron spin (Figure 112). The Rashba field, would act as an in-plane field favouring a Bloch configuration, however not driving it forward. The effective Rashba field, $\mathrm{H}_{\mathrm{R}}$, is given according to the relation [57],

$$
H_{R}=-\frac{2 \alpha_{R} m_{e}}{\hbar|e| M_{S}} P\left|J_{e}\right|\left(\hat{z} \times J_{e}\right)
$$




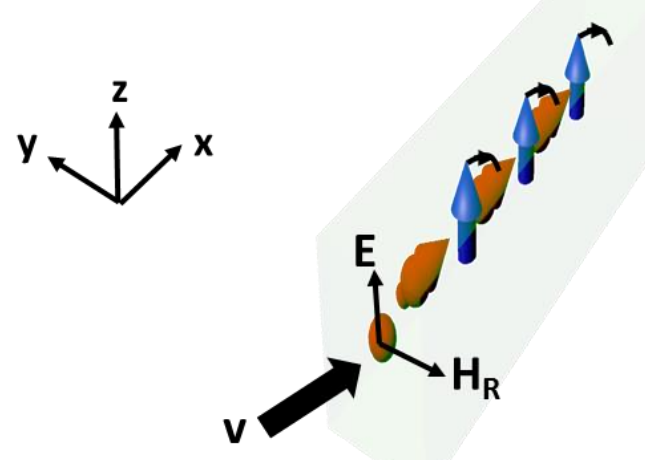

Figure 1-12 Schematic illustrating Rashba effect in an out-of-plane magnetized nanostrip. The electrons with velocity $\mathrm{v}$ see the electric field due to crystal potential which acts as Rashba magnetic field $\left(\mathrm{H}_{\mathrm{R}}\right)$ in their rest frame. This tilts the moments slightly out-of-plane due to s-d interactions.

where, $\alpha_{R}$ is the Rashba parameter, $\mathrm{m}_{\mathrm{e}}$ is the electron mass and $J_{e}$ is in the electron flow direction. Miron et al. demonstrated fast DW motion $(\sim 400 \mathrm{~m} / \mathrm{s})$ in $\mathrm{Pt} / \mathrm{Co} / \mathrm{AlO}_{\mathrm{x}}$ strips along the current flow direction [23]. They attributed Rashba effect and negative non-adiabatic torque as the driving mechanism.

More recent studies by Liu et al. on similar magnetic stacks attribute the SOT to originate primarily due to spin Hall effect (SHE) $[7,8,58]$. The SHE would cause the electrons with one spin polarization direction to accumulate at the ferromagnet/heavy metal interface. The accumulated spins would then exert a torque on the magnetization of the ferromagnetic layer. The torque due to SHE is given by the relation [26],

$$
\tau_{S H E}=-|\gamma| \boldsymbol{m} \times\left(\boldsymbol{m} \times H_{S L}\left(\hat{z} \times J_{e}\right)\right)
$$

$\mathrm{H}_{\mathrm{SL}}$ is the Slonczewski-like field and is given by the following relation [26],

$$
\mathbf{H}_{\mathbf{S L}}=\frac{\hbar \theta_{s h}\left|\mathbf{J}_{\mathbf{e}}\right|}{2|e| M_{S} t_{f}}
$$


where, $t_{f}$ is the thickness of the ferromagnetic layer and $\theta_{s h}$ is the spin Hall angle or the ratio of spin current to charge current. Figure 1-13 shows the schematic of SOT due to SHE. It is worth noting that the SOT from SHE cannot rotate the spins out-of-plane if the DW is Bloch type, since it lacks the correct symmetry [25]. Thus to drive the DWs an additional torque term is required which stabilizes Néel DWs.

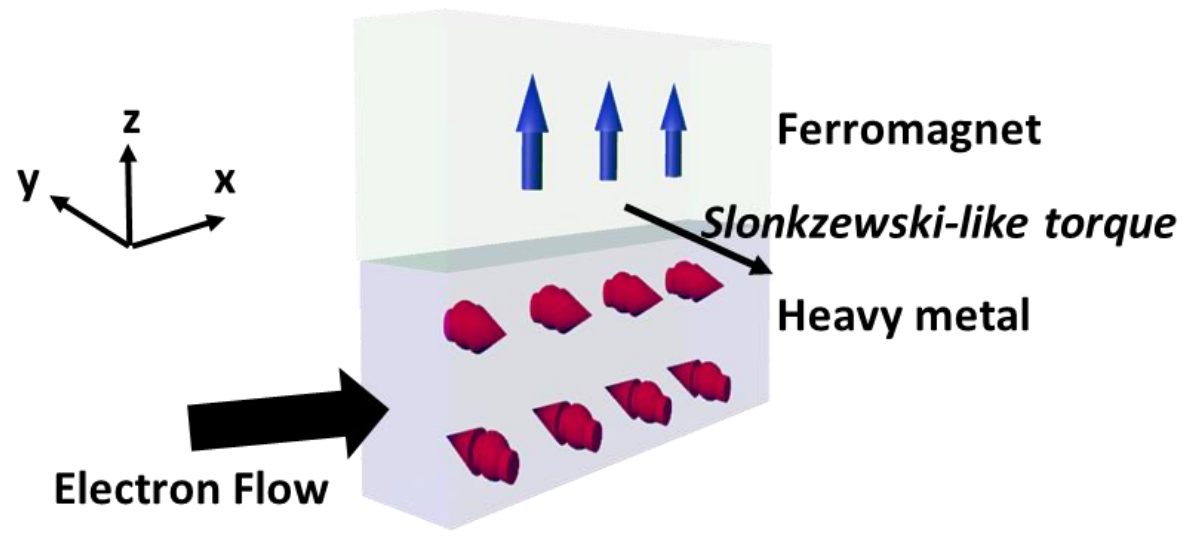

Figure 1-13 Schematic illustrating spin Hall effect induced spin-orbit torque. The Slonczewskilike or the anti-damping torque is also shown. Adapted from [25]

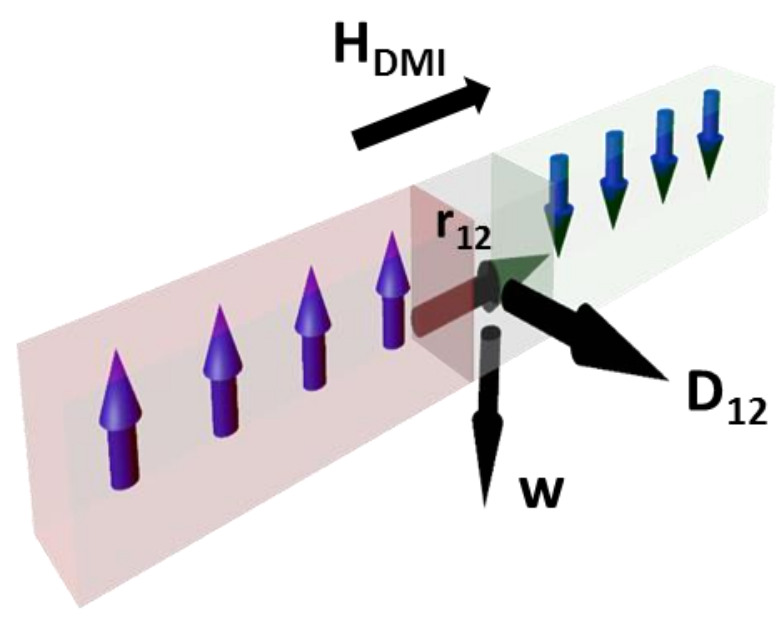

Figure 1-14 Schematic illustrating Dzyaloshinskii-Moriya (DMI) vector acting on spins due anti-symmetric exchange interaction. The DMI field favours Néel DWs. Adapted from [44] 
In systems with broken inversion symmetry and strong spin-orbit coupling, an antisymmetric exchange interaction namely, Dzyloshinskii-Moriya interaction (DMI) [21, 22] is present which prefers non-collinear spin alignment and non-uniform magnetic structures [59, 60]. DMI also favours the existence of Néel DWs over Bloch DWs [61]. Consider two spins $\mathbf{S}_{\mathbf{1}}$ and $\mathbf{S}_{\mathbf{2}}$ representing domain magnetizations which are separated by distance $\mathbf{r}_{\mathbf{1 2}}$, let w be a vector parallel to the wall plane as shown in Figure 1-14. DMI vector can be defined as,

$$
\boldsymbol{D}_{12}=\delta_{S O} \boldsymbol{r}_{12} \times \boldsymbol{w}
$$

and the DMI interaction energy is expressed as [62, 63],

$$
E_{D M}=D_{12} \cdot S_{1} \times S_{2}
$$

Thus $\boldsymbol{D}_{12}$ vector is orthogonal to the plane containing the spins and the interaction energy favours a chiral Néel DW which depends on the sign of $\delta_{S O}$, a coefficient proportional to spin-orbit coupling [26]. The orientation of the $\boldsymbol{D}_{12}$ vector is opposite for up-down and down-up DW configurations. The field induced by DMI is collinear to $\mathbf{r}_{\mathbf{1 2}}$, and is represented as,

$$
\boldsymbol{H}_{D M I}=-\frac{\partial E_{D M}}{\partial \boldsymbol{m}}
$$

The DMI field, $\boldsymbol{H}_{\boldsymbol{D M I}}$ can be expressed in terms of $\boldsymbol{D}$ and the domain wall width, $\Delta[64,65]$

$$
\boldsymbol{H}_{D M I}=\frac{\boldsymbol{D}}{\mu_{0} M_{S} \Delta}
$$

The above equation can be used to estimate the DMI coefficient. There exists a minimum critical value of $\mathrm{H}_{\mathrm{DMI}}$ such that it is able to overcome the magnetostatic energy and favour Néel DWs. Consider $\boldsymbol{H}_{\boldsymbol{D}}$ as the DW anisotropy field such that, $\boldsymbol{H}_{\boldsymbol{D}}=4 \boldsymbol{K}_{\boldsymbol{D}} / \pi \mu_{0} \boldsymbol{M}_{S}$, with $\boldsymbol{K}_{\boldsymbol{D}}=$ $N_{x} \mu_{0} M_{S}^{2} / 2$ being the magnetostatic DW anisotropy and $N_{x}=t_{f} \ln (2) / \pi \Delta$ is the demagnetization coefficient for magnetic film of thickness $t_{f}[34]$. Then Néel DWs are preferred if $\mathrm{H}_{\mathrm{DMI}}>\mathrm{H}_{\mathrm{D}}$ else Bloch DWs are stabilized in the thin films $[64,66]$. 
Consider the presence of DMI field torque together with the SHE induced SOT. When no current is applied the DW is Néel type and the magnetization in the DW is parallel to HDMI. When current is applied in the heavy metal underlayer, the spin polarized electrons accumulated near the ferromagnet would exert a torque and rotate the magnetization in the wall from longitudinal to transverse i.e. Néel to Bloch. This creates a finite angle between magnetization and $\mathrm{H}_{\mathrm{DMI}}$, thus this exerts a torque rotating the magnetization out-of-plane and driving the DWs. The DWs can be driven along or against the current flow direction depending upon the sign of the spin Hall angle of the underlayer and the handedness or chirality of the Néel wall. Figure 1-15 (a-d) shows the sense of rotation of up-down and down-up Néel DWs. If the spin Hall angle of underlayer is positive then left handed Néel

(a)

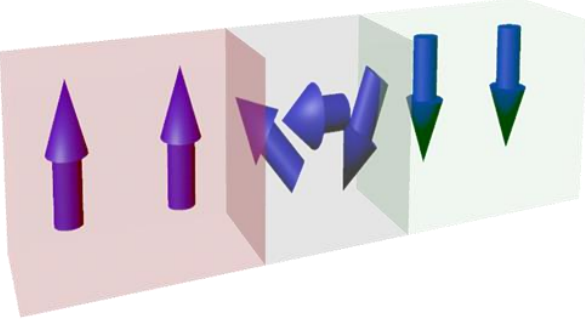

(c)

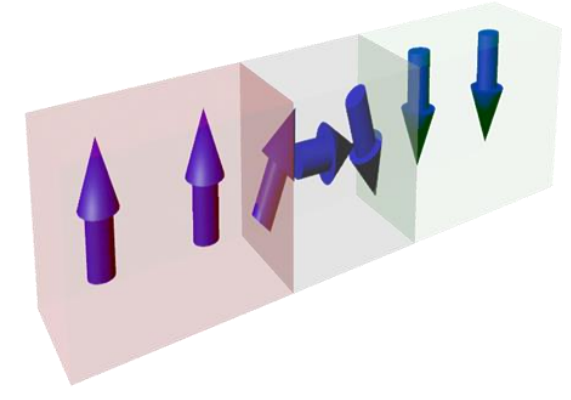

(b)

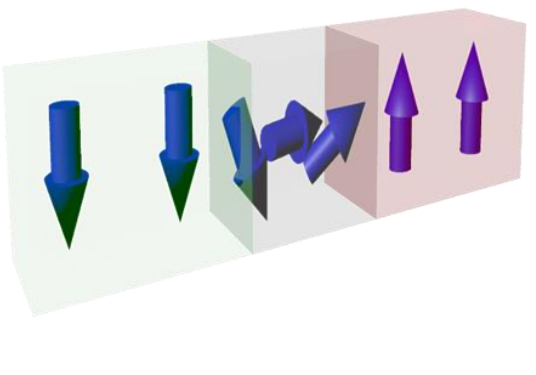

(d)

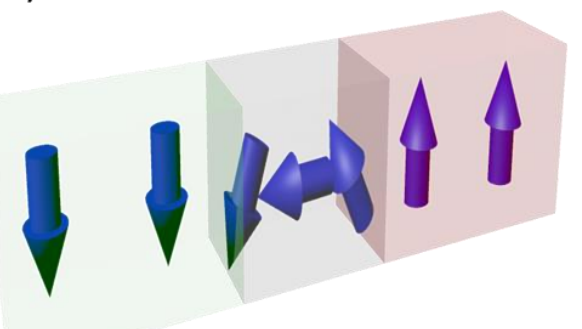

Figure 1-15 Schematic depicting sense of rotation of chiral Néel DWs, which is clockwise for right handed and anti-clockwise for left handed (a) Up-Down Left handed (b) Down-Up Left handed (c) Up-Down Right handed (d) Down-Up Right handed. Adapted from [24] 
wall propagates along the current flow direction and right handed propagates along the electron flow direction. The directions reverse if the spin Hall angle becomes negative. As per convention, the DW is said to be left handed if the spins rotate counter-clockwise going from left domain to the right domain and is said to be right handed if the sense of rotation is clockwise [24, 67, 68].

The combined LLG equation incorporating the effect of field, STT, SOT and DMI can be written as follows [69]:

$$
\frac{\partial \boldsymbol{m}}{\partial t}=-|\gamma| \boldsymbol{m} \times \boldsymbol{H}_{e f f}+\alpha \boldsymbol{m} \times \frac{\partial \boldsymbol{m}}{\partial t}-(\boldsymbol{u} \cdot \nabla) \boldsymbol{m}-\beta \boldsymbol{m} \times[-(\boldsymbol{u} \cdot \nabla) \boldsymbol{m}]-|\gamma| \boldsymbol{m} \times\left[\boldsymbol{m} \times\left|H_{S L}\right|\left(\hat{z} \times \boldsymbol{J}_{\boldsymbol{e}}\right)\right]
$$

where, $\boldsymbol{H}_{\text {eff }}$ along with including Zeeman, magnetostatic, exchange and anisotropy, also has contribution from $\mathrm{H}_{\mathrm{DMI}}$, the third and fourth terms are torques due to STT and SOT, respectively. Here, the Rashba effect and field like term is excluded since its contribution is small $[7,8,26]$.

\subsection{Review of spintronic logic devices}

Charge based logic schemes utilize the presence or absence of charge to distinguish between logic states 0 and 1 . The switching device e.g. CMOS, can be considered as two potential wells separating the charge using an adjustable barrier. Theoretical studies have found the minimum switching energy between the two states as $\mathrm{k}_{\mathrm{B}} \mathrm{T} \ln 2(23 \mathrm{meV})$ [70]. However, the projected gate switching energy for $10 \mathrm{~nm}$ gate length is $15 \mathrm{eV}$ [71]. The information stored in the electron spin orientation is devoid of such switching energies. The non-volatility also eliminates the delay and the energy required to obtain information from the microprocessor while it is in the stand-by state, thus saving power needed to periodically turn the processor on or off. Numerous spintronic based logic devices have been proposed, however practical limitations such as direct cascading and small signal to noise ratio [72] prevent successful commercialization of the 
technology. In this section we review some of the proposed schemes of the spintronic based logic devices.

\subsubsection{Nano-magnetic logic}

Magnetic quantum cellular automata (MQCA) or nano-magnetic logic (NML) are built from identical magnetic dots which are elongated along one direction to form a group of elliptical nanomagnets. These nanomagnets interact through electromagnetic interactions which are either exchange or primarily dipole interactions. Room temperature demonstration of MQCA based logic was performed by Cowburn et al. [73]. Majority gate logic application of MQCA was demonstrated by Imre et al. [74] and their fabricated structures are shown in Figure 1-16

(a)
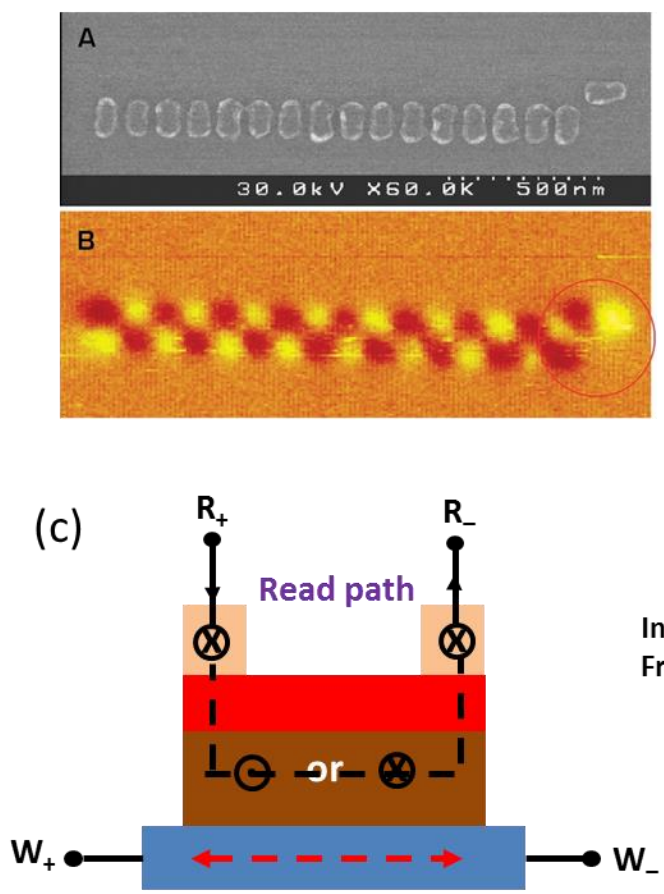

(b)

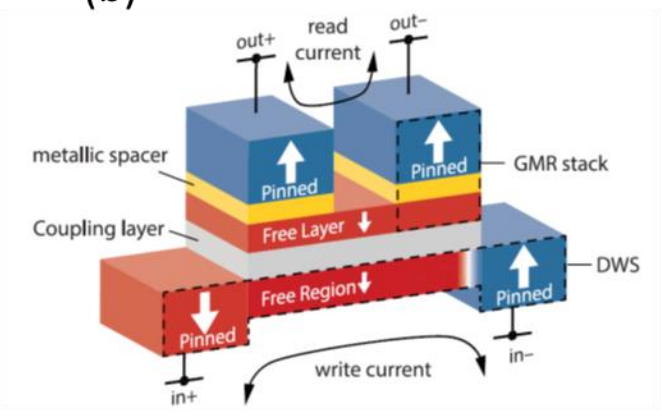

(d)

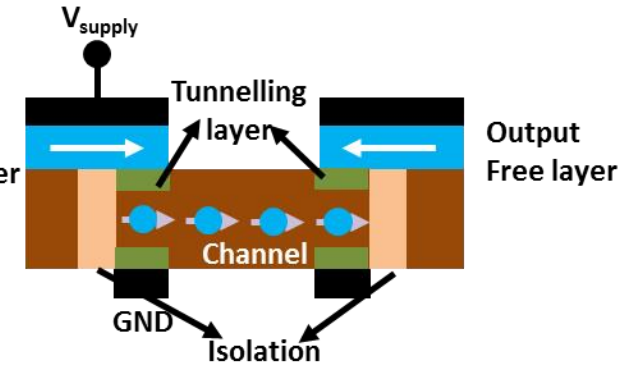

Figure 1-16 (a) Array of nanomagnets interacting via dipolar coupling, the horizontal nanomagnet on the right represents the input bit, the MFM imaging shows the antiferromagnetic coupling, adapted from [74]. (b) Schematic of an n-type all metallic logic device, with displacement of DW transferring the information to the free layer of the coupled spin-valve. The output is connected to the pinned layer of the spin-valve, adapted from [75]. (c) Schematic of spin Hall effect-MTJ based four terminal logic device, the read path and the write path are decoupled providing non-reciprocity, redrawn from [76]. (d) Schematic of all spin logic device, the input nanomagnet injects spin current into a metallic channel which is relayed to the output nanomagnet through spin-torque, redrawn from [77]. 
(a). The neighbouring magnets would adopt anti-parallel magnetization due to dipolar coupling as shown in the MFM images. The singular magnet placed horizontally on the right corner would act as the control bit which can control the magnetization direction of the other magnets. By different arrangement of the nanomagnets logic gate operations can be obtained. However, all the logic operations require the need of external magnetic field for clocking, which is an issue for on-chip integration and scalability. Recently, Bhowmik et al. [78], proposed the utilization of spin Hall effect to control the logic bits of NML with perpendicular anisotropy. The researchers showed that if the current is made to flow thorough a thick Ta layer underneath the nanomagnets it could induce the switching through spin-orbit torque phenomenon. The current flow direction needs to be designed such that it does not exert torque on the input nanomagnet. The limitation of this approach is the excess joule heating caused by the current flow through the highly resistive Ta layer. Although NML technology offers fast switching speeds, the dipole interaction between the magnets is sensitive to shape. The single domain states also becomes unstable due to thermal effects.

\subsubsection{STT-MTJ based logic}

Many variations of STT-MTJ based logic schemes have been proposed. An all metallic logic scheme, referred to as mLogic was proposed by Morris et al. [79]. Figure 1-16 (b) shows the schematic of an mLogic device. It consists of two GMR or MTJ stacks for reading the output state, which is coupled to a free regions carrying the input data. The input is applied by passing current across the free layer through two pinned layers. Domain wall (DW) exists at one end which carries the information. When a current flows from the positive input to the negative input, the DW propagates towards the left and switches the free region of the spin valve to an up magnetized state. Since the pinned region is also up-magnetized the resistance is low in this state and this corresponds to logic bit ' 0 '. On the other hand when the current flows in the 
opposite direction, the resistance remains in the high state and this corresponds to logic bit ' 1 '. Different combinations can be realized by changing the initial spin orientation of the pinned layer. The low resistance path in the all-metallic path allows operation at low voltages, but it also provides a leakage path which could worsen the energy efficiency as compared to CMOS logic.

STT based logic schemes would have practical issues like high power consumption due to the requirement of large critical current to switch the magnetization. The reliability is also a concern due to breakdown of tunnel barrier on repeated switching cycles. To overcome these issues spin Hall effect (SHE) based MTJ logic devices have been proposed $[12,80]$. In three terminal SHE-MTJ device the free layer is in direct contact with the heavy metal layer. This overcomes the high write current and tunnel barrier degradation since the read and write paths are separate. However, it is difficult to cascade this three terminal device for pipeline computing. This issue can be solved by considering a four-terminal device as shown in Figure 1-16 (c), proposed by Kang et al. [76]. Compared to three terminal device, the four terminal device has an additional read terminal. The fully decoupled read and write paths eliminate the feedback to the input, hence enabling direct cascading. The issue with SHE-MTJ logic schemes is low density due to limitation in scaling down the device geometry and additional overheads.

\subsubsection{All spin logic}

The logic devices which combine charge based and spin based mechanisms such as semiconductor spintronic logic devices suffer from dynamic power dissipation due to frequent conversion from electrical to magnetic state. All spin logic (ASL) overcomes the above challenges since it computes and stores the information in the magnetic state [77]. ASL employs non-local spin signal to switch a nanomagnet constituting input to the next stage. Figure 1-16 (d) shows the schematic of the ASL device proposed by Behin-Aein et al. [77]. 
The input and output nanomagnets are free layers and can switch left or right representing the binary data bits. The magnetization of the input magnet generates a spin current which propagates in the channel towards the output nanomagnet. The output nanomagnet can in-turn be switched by this spin current due to the application of spin torque. Thus by applying a negative voltage to the input, the majority spins can be injected and the information is 'copied' to the output. By reversing the polarity of the input voltage to positive, the majority spins are attracted and their absence aligns the output nanomagnet antiparallel to the input nanomagnet, thus providing a 'NOT' gate operation. The conducting channel could be a metal or semiconductor. Although the semiconductors exhibit longer spin coherence lengths [81-84], the injection efficiency is low. Another important aspect is that the switching process should be non-reciprocal to prevent the output state from affecting the input state. This can be accomplished to have input side with a tunnelling interface which would enhance the injection efficiency in a metal or a semiconductor [85-90], and an output side with a low resistance interface to suppress the back injection.

An advantage of ASL over CMOS logic is that the logic gates can be implemented more compactly. There are some practical limitations of the ASL device. For instance the limitation of spin flip length in the metallic channel requires the use of repeaters such as nanomagnets to transmit information. These can consume significant power in the actual design. Scaling down ASL can increase the efficiency of spin-torque on the output due to less spin-flip scattering, however as the input and output become closer, the dipole interaction between the magnets would start to create interference. There is a leakage current in the channel while cascading which would increase on scaling down the device further. The spins could also flow into the ground due to reduction of the shunt path. 
Table 1-1. Comparison of device parameters for CMOS and spintronic based logic schemes [91]

\begin{tabular}{|c|c|c|c|c|c|c|}
\hline Device name & $\begin{array}{c}\text { Energy } \\
(\mathrm{fJ})\end{array}$ & $\begin{array}{c}\text { Delay } \\
(\mathrm{ps})\end{array}$ & $\begin{array}{c}\text { Active } \\
\text { power } \\
(\mathrm{W})\end{array}$ & $\begin{array}{c}\text { Stand-by } \\
\text { power } \\
(\mathrm{W})\end{array}$ & $\begin{array}{c}\text { Power } \\
\text { Density } \\
\left(\mathrm{W} / \mathrm{cm}^{2}\right)\end{array}$ & $\begin{array}{c}\text { Throughput } \\
\left(/ \mathrm{s}-\mathrm{cm}^{2}\right)\end{array}$ \\
\hline $\begin{array}{c}\text { CMOS high } \\
\text { performance }\end{array}$ & $10^{2}$ & $10^{3}$ & $10^{-4}$ & $10^{-5}-10^{-6}$ & 10 & $10^{2}$ \\
\hline CMOS low voltage & 10 & $10^{4}$ & $10^{-6}$ & $10^{-8}-10^{-7}$ & 1 & $10-10^{2}$ \\
\hline All-spin logic & $10^{4}$ & $10^{5}-10^{6}$ & $10^{-5}$ & $10^{-8}$ & 10 & 1 \\
\hline STT- MTJ/Domain- & $10^{2}$ & $10^{5}-10^{6}$ & $10^{-5}-10^{-6}$ & $10^{-8}$ & $1-10$ & $1-10$ \\
\hline wall & $10^{2}$ & $10^{5}-10^{6}$ & $10^{-7}$ & $10^{-10}$ & $1-10^{-1}$ & 10 \\
\hline Nano-magnetic logic & & & & & 10 \\
\hline
\end{tabular}

Table 1-1, shows a comparison of device parameters for CMOS and spintronic based logic schemes. The benchmarking was carried out by Nikonov et al. [91] by adopting an electrical model for spin-based designs in 32 bit arithmetic logic unit (ALU) and adder circuits. The numerical values in the table indicate the computed order of magnitude of the device parameters for respective technologies. The data reveals than the spintronic devices in general switch slower and consume more energy per switching than the CMOS based devices. The advantages are low dynamic and static power consumption which compensates for the low throughput. Thus, although the spintronic logic has potential, more research is required to make its performance competitive with that of CMOS based schemes.

The review presents the advantages and challenges in the existing spintronic logic schemes. The application purpose would dictate which logic scheme is suitable. The next section gives a brief review on the existing memory and logic devices exploiting DW dynamics in ferromagnetic structures. 


\subsection{Existing memory and logic schemes based on domain-wall dynamics}

Both field and current induced DW dynamics can be exploited to realize memory and logic applications. The magnetization orientation of the region enclosed by the DW can be assigned logic bit 1 or 0 in the case of PMA strips. For IMA strips the DW chirality and the magnetization directions of the conduit can serve as the logic bit.

The first demonstration of DW based logic device was shown by Allwood et al. [19]. Figure 1-17 (a) shows the structure proposed for logic operations. The shape anisotropy of the nanostructure constrains the magnetization to follow the path of the conduit. Time varying magnetic fields were applied along the vertical and horizontal directions and MOKE signal was traced at various junctions. The bifurcation of DWs at the fan out and their combination gives rise to logic operations, for instance the MOKE signal is high at IV only when it is high at both II and III. Thus output at IV is logical AND of inputs at II and III. The application of DW in memory devices was proposed by Parkin et al. [14] and termed as racetrack memory. In a racetrack, the bits are stored as alternate regions of magnetization in a nanowire as shown in Figure 1-17 (b). The bits can be written at one end of the nanowire by using a current carrying conductor to generate localized Oersted field. The bits can be pushed further in the nanowire by using spin polarized current. The bits can also be confined in a region by using notches to pin the DW. The reading of data can be performed by placing a magnetoresistive tunnel junction at one end of the nanowire. The racetrack device can also be used as a shift register [92]. The racetrack memory can be constructed in three dimensions to increase the device density, however the fabrication of such a structure is challenging.

Chiba et al. [93], proposed a PMA racetrack using a Co/Ni nanowire as shown in Figure 117 (c). The stripline is used for writing bits. One bit is written by flowing current in the stripline in one direction. Then multiple pulses are applied to the nanowire to drive the DW. Then to write another bit, the current in the stripline is reversed. The bits are read using a Hall sensor 

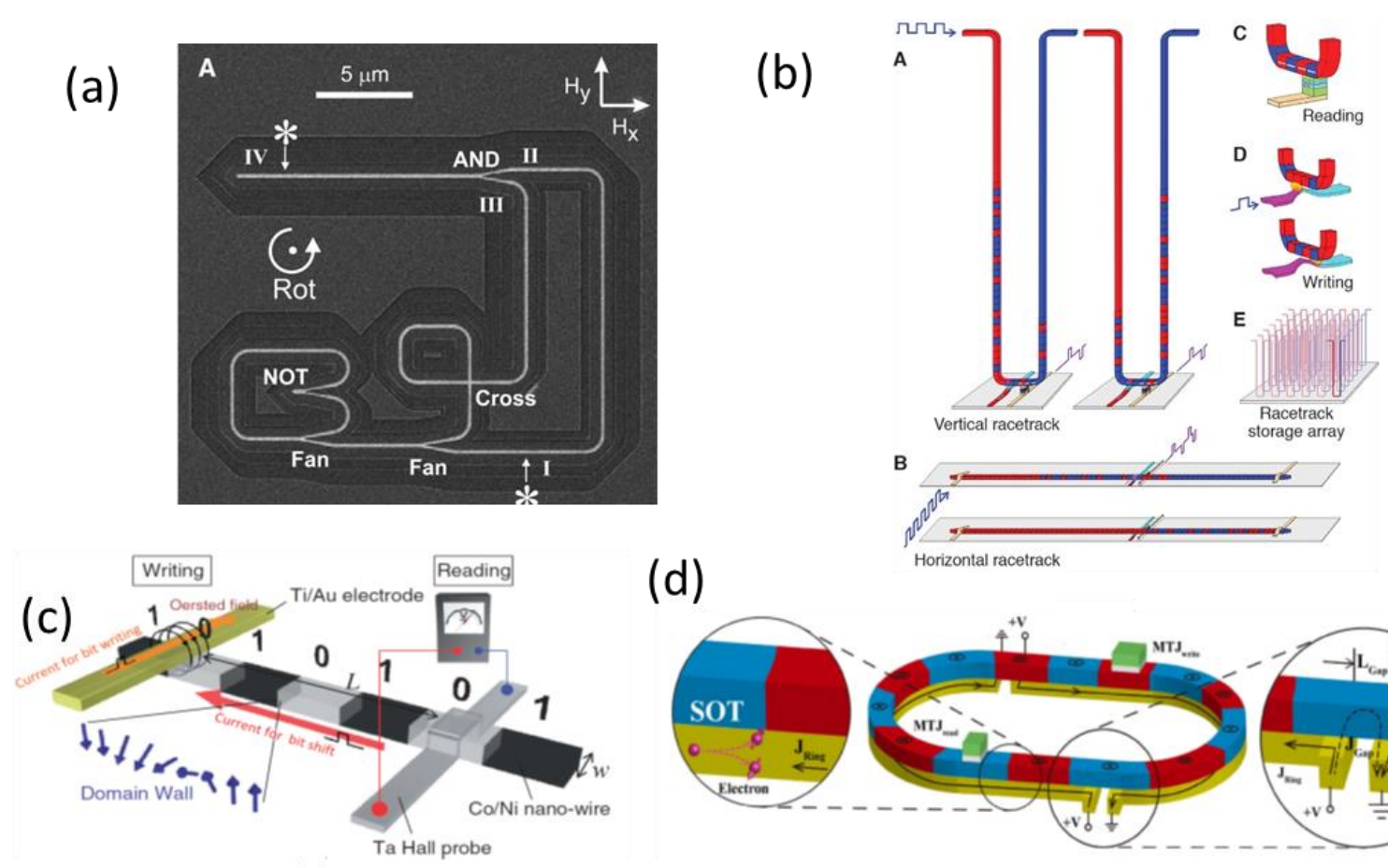

(d)

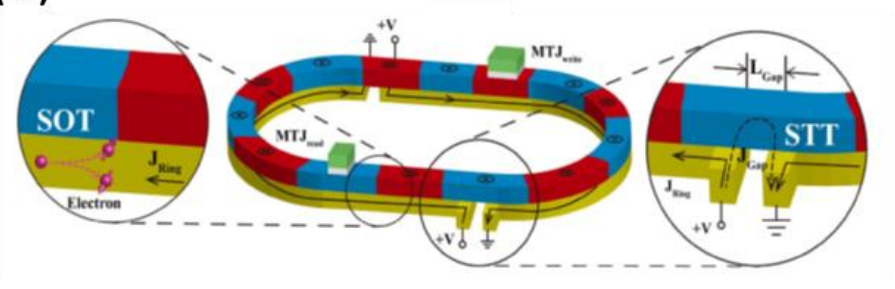

Figure 1-17 Review of DW devices (a) SEM image of magnetic DW logic device, the rotating magnetic field propels the DW along the circuit. The geometry of the nanostructure provides different logic combinations, adapted from [19] (b) Schematic of racetrack memory device. The information is stored in a nanowire as alternate regions of magnetization, the bits can be transferred by applying a current to induce STT and the read using read sensor such as MTJ, adapted from [14] (c) Schematic of PMA racetrack memory, the bits are written by applying a local Oersted field generated through a current carrying stripline. The output can be detected at the Hall probe and bits can propagate through application of current, adapted from [93] (d) Schematic of ring shaped racetrack memory, where the DWs are driven by SOT. The current passes through the heavy metal layer and exerts the SOT on the DWs. Short circuit is prevented by breaking the heavy-metal contact locally and the current continuity maintained by leakage current propagating through the ferromagnet, adapted from [94].

patterned at the other extreme of the nanowire. Kim et al. [95] showed the dependence of DW speed on the strength of perpendicular anisotropy of the nanowire in $\mathrm{Pt} / \mathrm{Co} / \mathrm{Pt}$ stack. It was observed that the speed depends on the thickness of the Pt layer. When the thickness of underlayer is not sufficient to induce strong PMA, the speed is also higher. The dependence of speed on thickness of underlayer and capping layer Pt follows the same trend as the anisotropy constant. Thus speed is higher when anisotropy constant is lower. Yang et al. [96], demonstrated DW velocities of the order of $750 \mathrm{~m} / \mathrm{s}$ in synthetic anti-ferromagnetically coupled 
PMA nanowires. The enhancement in velocity was attributed to the combination of DMI field torque and the exchange coupled torque due to the anti-ferromagnetic coupling. The velocity was found to be maximum when antiferromagnetic coupling was strong so as to reduce net magnetization to near zero. The antiferromagnetic coupling also minimizes dipolar coupling which enables higher device density. The only drawback is the current density required to achieve such an order of speed is relatively high $\left(\sim 3 \times 10^{12} \mathrm{~A} / \mathrm{m}^{2}\right)$ for device applications. Metaxas et al. [97], employed a low current density $\left(\sim 5 \times 10^{8} \mathrm{~A} / \mathrm{m}^{2}\right)$ to drive DWs with velocity as high a $400 \mathrm{~m} / \mathrm{s}$ in the free layer of an in-plane magnetic tunnel junction (MTJ). A vertical current injection method [98] was employed, wherein, the injected current is spin polarized by passing it through the fixed layer of the MTJ. This current drives the DW which is present in the free layer. However, the displacement of the DW was confined to a few nanometers. The racetrack memory which requires bi-directionality of DW motion could lead to data overflow in the case of linear nanowire. To address this issue, Zhang et al. [94] have proposed a ring shaped racetrack memory, in which the DWs are driven by SOT. In this set-up, the current is passed through the heavy metal under layer only. To avoid short-circuit, the racetrack is opened at two ends as shown in Figure 1-17 (d). Simulations reveal that current can leak through the ferromagnetic region where the heavy metal layer is open, this could drive the DW via STT and maintain circuit continuity.

\subsection{Organization of this Thesis}

Chapter 1 is an introduction and theoretical overview of ferromagnetism and domain wall dynamics. The dynamics involving field induced motion, spin-transfer and spin-orbit torque are explained. Brief review of work on spintronic logic and domain wall based devices is discussed. 
Chapter 2 outlines the various experimental and characterization techniques employed in this work and their basic operating principle.

Chapter 3 reveals the chirality dependent domain wall trajectory in in-plane ferromagnetic network structures. Geometrical asymmetry is discussed to overcome Walker breakdown issue by changing the potential landscape.

Chapter 4 demonstrates a prototype of domain wall based programmable logic device utilizing geometrical asymmetry and current carrying gate to control the path of the domain wall.

Chapter 5 is a study of domain wall dynamics in $\mathrm{Co} / \mathrm{Ni}$ nanowires with perpendicular magnetic anisotropy. The study reveals in-plane current induced stochastic domain wall nucleation in the nanostrips. A random number generator device is also demonstrated using an external chip for signal amplification and digitization.

Chapter 6 explores domain wall dynamics using spin-orbit torque in $\mathrm{Co} / \mathrm{Pt}$ double stack structures. Hard-axis field is applied to support and oppose the inherent Dzyloshinskii-Moriya interaction field and high speed bi-directional motion is demonstrated on tuning the heavymetal thicknesses.

Chapter 7 summarizes the key findings in this work and discusses possible future directions.

Appendix A discusses additional work carried out to support key findings in Chapter 6 regarding spin-orbit torque induced domain wall dynamics in Co/Pt double stack structures. 


\section{Bibliography}

[1] G. E. Moore, "Cramming More Components onto Integrated Circuits," Electronics, pp. 114$117,1965$.

[2] S. Borkar and A. A. Chien, "The Future of Microprocessors," Communications of the Acm, vol. 54, pp. 67-77, May 2011.

[3] M. M. Waldrop, "More than Moore," Nature, vol. 530, pp. 145-147, 2016.

[4] L. Berger, "Emission of spin waves by a magnetic multilayer traversed by a current," Physical Review B, vol. 54, pp. 9353-9358, Oct 11996.

[5] J. C. Slonczewski, "Current-driven excitation of magnetic multilayers," Journal of Magnetism and Magnetic Materials, vol. 159, pp. L1-L7, Jun 1996.

[6] I. M. Miron, K. Garello, G. Gaudin, P. J. Zermatten, M. V. Costache, S. Auffret, et al., "Perpendicular switching of a single ferromagnetic layer induced by in-plane current injection," Nature, vol. 476, pp. 189-93, Aug 112011.

[7] L. Q. Liu, O. J. Lee, T. J. Gudmundsen, D. C. Ralph, and R. A. Buhrman, "Current-Induced Switching of Perpendicularly Magnetized Magnetic Layers Using Spin Torque from the Spin Hall Effect," Physical Review Letters, vol. 109, Aug 292012.

[8] L. Liu, C. F. Pai, Y. Li, H. W. Tseng, D. C. Ralph, and R. A. Buhrman, "Spin-torque switching with the giant spin Hall effect of tantalum," Science, vol. 336, pp. 555-8, May 042012.

[9] A. D. Kent and D. C. Worledge, "A new spin on magnetic memories," Nature Nanotechnology, vol. 10, pp. 187-191, Mar 152015.

[10] J. Liu, B. Jaiyen, R. Veras, and O. Mutlu, "RAIDR: Retention-Aware Intelligent DRAM Refresh," Proceedings of the 39th Annual International Symposium on Computer Architecture ISCA'12, pp. 1-12, 2012.

[11] T. Min, Q. Chen, R. Beach, G. Jan, C. Horng, W. Kula, et al., "A Study of Write Margin of Spin Torque Transfer Magnetic Random Access Memory Technology," Ieee Transactions on Magnetics, vol. 46, pp. 2322-2327, 2010.

[12] M. Cubukcu, O. Boulle, M. Drouard, K. Garello, C. O. Avci, I. M. Miron, et al., "Spin-orbit torque magnetization switching of a three-terminal perpendicular magnetic tunnel junction," Applied Physics Letters, vol. 104, p. 042406, 2014.

[13] G. Prenat, K. Jabeur, P. Vanhauwaert, G. D. Pendina, F. Oboril, R. Bishnoi, et al., "Ultra-Fast and High-Reliability SOT-MRAM: From Cache Replacement to Normally-Off Computing," IEEE Transactions on Multi-Scale Computing Systems, vol. 2, pp. 49-60, 2016.

[14] S. S. Parkin, M. Hayashi, and L. Thomas, "Magnetic domain-wall racetrack memory," Science, vol. 320, pp. 190-4, Apr 112008.

[15] A. J. Annunziata, M. C. Gaidis, L. Thomas, C. W. Chien, C. C. Hung, P. Chevalier, et al., "Racetrack memory cell array with integrated magnetic tunnel junction readout," in Technical Digest - International Electron Devices Meeting, IEDM, 2011, pp. 24.3.1-24.3.4.

[16] Y. Zhang, W. Zhao, J. O. Klein, D. Ravelsona, and C. Chappert, "Ultra-high density content addressable memory based on current induced domain wall motion in magnetic track," IEEE Transactions on Magnetics, vol. 48, pp. 3219-3222, 2012.

[17] S. Parkin and S. H. Yang, "Memory on the racetrack," Nature Nanotechnology, vol. 10, pp. 195-198, 2015.

[18] D. A. Allwood, G. Xiong, M. D. Cooke, C. C. Faulkner, D. Atkinson, N. Vernier, et al., "Submicrometer ferromagnetic NOT gate and shift register," Science, vol. 296, pp. 2003-2006, Jun 142002.

[19] D. A. Allwood, G. Xiong, C. C. Faulkner, D. Atkinson, D. Petit, and R. P. Cowburn, "Magnetic domain-wall logic," Science, vol. 309, pp. 1688-92, Sep 092005.

[20] C. Murapaka, P. Sethi, S. Goolaup, and W. S. Lew, "Reconfigurable logic via gate controlled domain wall trajectory in magnetic network structure," Sci Rep, vol. 6, p. 20130, Feb 032016.

[21] I. E. Dzyaloshinskii, "On the Magneto-Electrical Effect in Antiferromagnets," Soviet Physics Jetp-Ussr, vol. 10, pp. 628-629, 1960. 
[22] T. Moriya, "Anisotropic Superexchange Interaction and Weak Ferromagnetism," Physical Review, vol. 120, pp. 91-98, 1960.

[23] I. M. Miron, T. Moore, H. Szambolics, L. D. Buda-Prejbeanu, S. Auffret, B. Rodmacq, et al., "Fast current-induced domain-wall motion controlled by the Rashba effect," Nat Mater, vol. 10, pp. 419-23, Jun 2011.

[24] S. Emori, U. Bauer, S. M. Ahn, E. Martinez, and G. S. Beach, "Current-driven dynamics of chiral ferromagnetic domain walls," Nat Mater, vol. 12, pp. 611-6, Jul 2013.

[25] P. P. Haazen, E. Mure, J. H. Franken, R. Lavrijsen, H. J. Swagten, and B. Koopmans, "Domain wall depinning governed by the spin Hall effect," Nat Mater, vol. 12, pp. 299-303, Apr 2013.

[26] A. V. Khvalkovskiy, V. Cros, D. Apalkov, V. Nikitin, M. Krounbi, K. A. Zvezdin, et al., "Matching domain-wall configuration and spin-orbit torques for efficient domain-wall motion," Physical Review B, vol. 87, Jan 42013.

[27] K. S. Ryu, L. Thomas, S. H. Yang, and S. Parkin, "Chiral spin torque at magnetic domain walls," Nature Nanotechnology, vol. 8, pp. 527-533, Jul 2013.

[28] S. Sachdev, "Quantum Phase Transitions," in Handbook of Magnetism and Advanced Magnetic Materials, H. Kronmuller and S. Parkin, Eds., ed: Wiley, 2007.

[29] J. Zhang, P. W. Hess, A. Kyprianidis, P. Becker, A. Lee, J. Smith, et al., "Observation of a descrete time crystal," Nature, vol. 543, pp. 217-220, 2017.

[30] C. Kittel, "Physical Theory of Ferromagnetic Domains," Reviews of Modern Physics, vol. 21, pp. 541-583, 1949.

[31] S. J. Barnett, "New researches on magnetization by rotation and the gyromagnetic ratios of ferromagnetic substances," Proceedings of the American Academy of Arts and Sciences, vol. 75, pp. 109-129, Aug-Dec 1944.

[32] C. Kittel, "On the Gyromagnetic Ratio and Spectroscopic Splitting Factor of Ferromagnetic Substances," Physical Review, vol. 76, pp. 743-748, 1949.

[33] J. M. Coey, Magnetism and magnetic materials: Cambridge University Press, 2010.

[34] S. V. Tarasenko, A. Stankiewicz, V. V. Tarasenko, and J. Ferre, "Bloch wall dynamics in ultrathin ferromagnetic films," Journal of Magnetism and Magnetic Materials, vol. 189, pp. 19-24, Oct 1998.

[35] A. Mougin, M. Cormier, J. Adam, P. Metaxas, and J. Ferré, "Domain wall mobility, stability and Walker breakdown in magnetic nanowires," EPL (Europhysics Letters), vol. 78, p. 57007, 2007.

[36] C. Tannous and J. Gieraltowski, "The Stoner-Wohlfarth model of ferromagnetism (vol 29, pg 475, 2008)," European Journal of Physics, vol. 30, pp. 227-227, Jan 2009.

[37] R. D. McMichael and M. J. Donahue, "Head to head domain wall structures in thin magnetic strips," Ieee Transactions on Magnetics, vol. 33, pp. 4167-4169, Sep 1997.

[38] T. Koyama, D. Chiba, K. Ueda, K. Kondou, H. Tanigawa, S. Fukami, et al., "Observation of the intrinsic pinning of a magnetic domain wall in a ferromagnetic nanowire," Nature Materials, vol. 10, pp. 194-197, Mar 2011.

[39] M. Lakshmanan and K. Nakamura, "Landau-Lifshitz equation of ferromagnetism: Exact treatment of the gilbert damping," Physical Review Letters, vol. 53, pp. 2497-2499, 1984.

[40] A. Thiaville, Y. Nakatani, J. Miltat, and Y. Suzuki, "Micromagnetic understanding of currentdriven domain wall motion in patterned nanowires," Europhysics Letters, vol. 69, pp. 990-996, Mar 2005.

[41] N. L. Schryer and L. R. Walker, "Motion of 180 Degrees Domain-Walls in Uniform Dc Magnetic-Fields," Journal of Applied Physics, vol. 45, pp. 5406-5421, 1974.

[42] G. S. D. Beach, C. Nistor, C. Knutson, M. Tsoi, and J. L. Erskine, "Dynamics of field-driven domain-wall propagation in ferromagnetic nanowires," Nature Materials, vol. 4, pp. 741-744, Oct 2005.

[43] G. S. D. Beach, M. Tsoi, and J. L. Erskine, "Current-induced domain wall motion," Journal of Magnetism and Magnetic Materials, vol. 320, pp. 1272-1281, Apr 2008.

[44] S.-H. Yang and S. Parkin, "Novel domain wall dynamics in synthetic antiferromagnets," Journal of physics. Condensed matter: an Institute of Physics journal, vol. 29, p. 303001, 2017.

[45] D. C. Langreth and J. W. Wilkins, "Theory of spin resonance in dilute magnetic alloys," Physical Review B, vol. 6, pp. 3189-3227, 1972. 
[46] G. Tatara and H. Kohno, "Theory of current-driven domain wall motion: Spin transfer versus momentum transfer," Physical Review Letters, vol. 92, Feb 272004.

[47] G. Tatara, T. Takayama, H. Kohno, J. Shibata, Y. Nakatani, and H. Fukuyama, "Threshold current of domain wall motion under extrinsic pinning, beta-term and non-adiabaticity," Journal of the Physical Society of Japan, vol. 75, Jun 2006.

[48] S. Zhang and Z. Li, "Roles of nonequilibrium conduction electrons on the magnetization dynamics of ferromagnets," Physical Review Letters, vol. 93, Sep 172004.

[49] G. Meier, M. Bolte, R. Eiselt, B. Kruger, D. H. Kim, and P. Fischer, "Direct imaging of stochastic domain-wall motion driven by nanosecond current pulses," Physical Review Letters, vol. 98, May 42007.

[50] R. Moriya, L. Thomas, M. Hayashi, Y. B. Bazaliy, C. Rettner, and S. S. P. Parkin, "Probing vortex-core dynamics using current-induced resonant excitation of a trapped domain wall," Nature Physics, vol. 4, pp. 368-372, May 2008.

[51] M. Eltschka, M. Wötzel, J. Rhensius, S. Krzyk, U. Nowak, M. Kläui, et al., "Nonadiabatic spin torque investigated using thermally activated magnetic domain wall dynamics," Physical Review Letters, vol. 105, 2010.

[52] L. Heyne, J. Rhensius, D. Ilgaz, A. Bisig, U. Rüdiger, M. Kläui, et al., "Direct determination of large spin-torque nonadiabaticity in vortex core dynamics," Physical Review Letters, vol. $105,2010$.

[53] J. E. Hirsch, "Spin Hall effect," Physical Review Letters, vol. 83, pp. 1834-1837, Aug 301999.

[54] N. Nagaosa, J. Sinova, S. Onoda, A. H. MacDonald, and N. P. Ong, "Anomalous Hall effect," Reviews of Modern Physics, vol. 82, pp. 1539-1592, May 132010.

[55] V. M. Edelstein, "Spin Polarization of Conduction Electrons Induced by Electric-Current in 2Dimensional Asymmetric Electron-Systems," Solid State Communications, vol. 73, pp. 233235, Jan 1990.

[56] C. R. Ast, J. Henk, A. Ernst, L. Moreschini, M. C. Falub, D. Pacile, et al., "Giant spin splitting through surface alloying," Physical Review Letters, vol. 98, May 42007.

[57] A. Manchon and S. Zhang, "Theory of nonequilibrium intrinsic spin torque in a single nanomagnet," Physical Review B, vol. 78, p. 212405, 2008.

[58] C. O. Avci, K. Garello, C. Nistor, S. Godey, B. Ballesteros, A. Mugarza, et al., "Fieldlike and antidamping spin-orbit torques in as-grown and annealed $\mathrm{Ta} / \mathrm{CoFeB} / \mathrm{MgO}$ layers," Physical Review B, vol. 89, Jun 232014.

[59] M. Bode, M. Heide, K. von Bergmann, P. Ferriani, S. Heinze, G. Bihlmayer, et al., "Chiral magnetic order at surfaces driven by inversion asymmetry," Nature, vol. 447, pp. 190-193, May 102007.

[60] A. Thiaville, S. Rohart, E. Jué, V. Cros, and A. Fert, "Dynamics of Dzyaloshinskii domain walls in ultrathin magnetic films," EPL, vol. 100, 2012.

[61] M. Heide, G. Bihlmayer, and S. Blugel, "Dzyaloshinskii-Moriya interaction accounting for the orientation of magnetic domains in ultrathin films: Fe/W(110)," Physical Review B, vol. 78, Oct 2008.

[62] A. Crepieux and C. Lacroix, "Dzyaloshinsky-Moriya interactions induced by symmetry breaking at a surface," Journal of Magnetism and Magnetic Materials, vol. 182, pp. 341-349, Feb 1998.

[63] A. Fert, V. Cros, and J. Sampaio, "Skyrmions on the track," Nature Nanotechnology, vol. 8, pp. 152-156, Mar 2013.

[64] R. Lavrijsen, D. M. F. Hartmann, A. Van Den Brink, Y. Yin, B. Barcones, R. A. Duine, et al., "Asymmetric magnetic bubble expansion under in-plane field in $\mathrm{Pt} / \mathrm{Co} / \mathrm{Pt}$ : Effect of interface engineering," Physical Review B - Condensed Matter and Materials Physics, vol. 91, 2015.

[65] R. Soucaille, M. Belmeguenai, J. Torrejon, J. V. Kim, T. Devolder, Y. Roussigné, et al., "Probing the Dzyaloshinskii-Moriya interaction in $\mathrm{CoFeB}$ ultrathin films using domain wall creep and Brillouin light spectroscopy," Physical Review B - Condensed Matter and Materials Physics, vol. 94, 2016.

[66] S. G. Je, D. H. Kim, S. C. Yoo, B. C. Min, K. J. Lee, and S. B. Choe, "Asymmetric magnetic domain-wall motion by the Dzyaloshinskii-Moriya interaction," Physical Review B, vol. 88, Dec 22013. 
[67] M. Belmeguenai, J.-P. Adam, Y. Roussigne, S. Eimer, T. Devolder, J.-V. Kim, et al., "Interfacial Dzyaloshinskii-Moriya interaction in perpendicularly magnetized $\mathrm{Pt} / \mathrm{Co} / \mathrm{AlOx}$ ultrathin films measured by Brillouin light spectroscopy," Physical Review B, vol. 91, 2015.

[68] O. Boulle, J. Vogel, H. Yang, S. Pizzini, D. de Souza Chaves, A. Locatelli, et al., "Roomtemperature chiral magnetic skyrmions in ultrathin magnetic nanostructures," Nat Nanotechnol, vol. 11, pp. 449-54, May 2016.

[69] E. Martinez, S. Emori, N. Perez, L. Torres, and G. S. Beach, "Current-driven dynamics of Dzyaloshinskii domain walls in the presence of in-plane fields: Full micromagnetic and onedimensional analysis," Journal of Applied Physics, vol. 115, p. 213909, 2014.

[70] R. Landauer, "Irreversibility and heat generation in the computing process," IBM journal of research and development, vol. 5, pp. 183-191, 1961.

[71] International Technology Roadmap for Semiconductors (Semiconductor Industry Association, San Jose California, USA, 2003) [Online]. Available: http://public.itrs.net

[72] J. S. Friedman and A. V. Sahakian, "Complementary magnetic tunnel junction logic," IEEE Transactions on Electron Devices, vol. 61, pp. 1207-1210, 2014.

[73] R. P. Cowburn and M. E. Welland, "Room Temperature Magnetic Quantum Cellular Automata," Science, vol. 287, pp. 1466-1468, 2000.

[74] A. Imre, G. Csaba, L. Ji, A. Orlov, G. H. Bernstein, and W. Porod, "Majority Logic Gate for Magnetic Quantum-Dot Cellular Automata," Science, vol. 311, pp. 205-208, 2006.

[75] X. Fong, Y. Kim, K. Yogendra, D. Fan, A. Sengupta, A. Raghunathan, et al., "Spin-transfer torque devices for logic and memory: Prospects and perspectives," IEEE Transactions on Computer-Aided Design of Integrated Circuits and Systems, vol. 35, pp. 1-22, 2016.

[76] W. Kang, Z. Wang, Y. Zhang, J.-O. Klein, W. Lv, and W. Zhao, "Spintronic logic design methodology based on spin Hall effect-driven magnetic tunnel junctions," Journal of Physics D: Applied Physics, vol. 49, p. 065008, 2016.

[77] B. Behin-Aein, D. Datta, S. Salahuddin, and S. Datta, "Proposal for an all-spin logic device with built-in memory," Nature Nanotechnology, vol. 5, pp. 266-270, 2010.

[78] D. Bhowmik, L. You, and S. Salahuddin, "Spin Hall effect clocking of nanomagnetic logic without a magnetic field," Nat. Nanotechnol., vol. 9, pp. 59-63, 2014.

[79] D. Morris, D. Bromberg, J.-G. J. Zhu, and L. Pileggi, "mLogic: Ultra-low voltage non-volatile logic circuits using STT-MTJ devices," in Proceedings of the 49th Annual Design Automation Conference, 2012, pp. 486-491.

[80] M. Yamanouchi, L. Chen, J. Kim, M. Hayashi, H. Sato, S. Fukami, et al., "Three terminal magnetic tunnel junction utilizing the spin Hall effect of iridium-doped copper," Applied Physics Letters, vol. 102, May 272013.

[81] B. Huang, D. J. Monsma, and I. Appelbaum, "Coherent spin transport through a 350 micron thick silicon wafer," Physical Review Letters, vol. 99, p. 177209, 2007.

[82] B. Huang and I. Appelbaum, "Spin dephasing in drift-dominated semiconductor spintronics devices," Physical Review B, vol. 77, p. 165331, 2008.

[83] B. Huang, H.-J. Jang, and I. Appelbaum, "Geometric dephasing-limited Hanle effect in longdistance lateral silicon spin transport devices," Applied Physics Letters, vol. 93, p. 162508, 2008.

[84] H.-J. Jang, J. Xu, J. Li, B. Huang, and I. Appelbaum, "Non-ohmic spin transport in n-type doped silicon," Physical Review B, vol. 78, p. 165329, 2008.

[85] E. Rashba, "Theory of electrical spin injection: Tunnel contacts as a solution of the conductivity mismatch problem," Physical Review B, vol. 62, p. R16267, 2000.

[86] G. Schmidt, D. Ferrand, L. Molenkamp, A. Filip, and B. Van Wees, "Fundamental obstacle for electrical spin injection from a ferromagnetic metal into a diffusive semiconductor," Physical Review B, vol. 62, p. R4790, 2000.

[87] I. Appelbaum, B. Huang, and D. J. Monsma, "Electronic measurement and control of spin transport in silicon," Nature, vol. 447, pp. 295-298, 2007.

[88] B. T. Jonker, G. Kioseoglou, A. T. Hanbicki, C. H. Li, and P. E. Thompson, "Electrical spininjection into silicon from a ferromagnetic metal/tunnel barrier contact," Nature Physics, vol. 3, p. 542, 2007. 
[89] X. Lou, C. Adelmann, S. A. Crooker, E. S. Garlid, J. Zhang, K. M. Reddy, et al., "Electrical detection of spin transport in lateral ferromagnet-semiconductor devices," Nature Physics, vol. 3, pp. 197-202, 2007.

[90] S. P. Dash, S. Sharma, R. S. Patel, M. P. de Jong, and R. Jansen, "Electrical creation of spin polarization in silicon at room temperature," Nature, vol. 462, pp. 491-494, 2009.

[91] D. E. Nikonov and I. A. Young, "Benchmarking of beyond-CMOS exploratory devices for logic integrated circuits," IEEE Journal on Exploratory Solid-State Computational Devices and Circuits, vol. 1, pp. 3-11, 2015.

[92] M. Hayashi, L. Thomas, R. Moriya, C. Rettner, and S. S. P. Parkin, "Current-controlled magnetic domain-wall nanowire shift register," Science, vol. 320, pp. 209-211, 2008.

[93] D. Chiba, G. Yamada, T. Koyama, K. Ueda, H. Tanigawa, S. Fukami, et al., "Control of multiple magnetic domain walls by current in a Co/Ni nano-wire," Applied Physics Express, vol. 3, 2010.

[94] Y. Zhang, X. Zhang, J. Hu, J. Nan, Z. Zheng, Z. Zhang, et al., "Ring-shaped Racetrack memory based on spin orbit torque driven chiral domain wall motions," Scientific Reports, vol. 6, 2016.

[95] D. H. Kim, S. C. Yoo, D. Y. Kim, K. W. Moon, S. G. Je, C. G. Cho, et al., "Maximizing domain-wall speed via magnetic anisotropy adjustment in $\mathrm{Pt} / \mathrm{Co} / \mathrm{Pt}$ films," Applied Physics Letters, vol. 104, 2014.

[96] S. H. Yang, K. S. Ryu, and S. Parkin, "Domain-wall velocities of up to $750 \mathrm{~m} \mathrm{~s}(-1)$ driven by exchange-coupling torque in synthetic antiferromagnets," Nature Nanotechnology, vol. 10, pp. 221-6, Mar 2015.

[97] P. J. Metaxas, J. Sampaio, A. Chanthbouala, R. Matsumoto, A. Anane, A. Fert, et al., "High domain wall velocities via spin transfer torque using vertical current injection," Scientific Reports, vol. 3, May 142013.

[98] A. Chanthbouala, R. Matsumoto, J. Grollier, V. Cros, A. Anane, A. Fert, et al., "Verticalcurrent-induced domain-wall motion in $\mathrm{MgO}$-based magnetic tunnel junctions with low current densities," Nature Physics, vol. 7, pp. 626-630, 2011. 


\section{Chapter 2}

\section{Experimental Methods}

This chapter discusses the experimental techniques employed in this research work. Thin films were deposited using the technique of magnetron sputtering. The deposited films were characterized for magnetic properties using alternating gradient force magnetometry (AGFM) and magneto-optical Kerr effect (MOKE) methods. The devices were patterned using electron beam lithography (EBL) and Ar-ion milling techniques in a top-down approach. The contact pads were patterned using EBL and subsequent lift-off in a bottom-up approach. The direct observation of domain wall (DW) dynamics were investigated using magnetic force microscopy and Kerr imaging methods. The devices were characterized electrically using a radio-frequency $(\mathrm{RF})$ probe station.

\subsection{Thin Film Deposition Process}

\subsubsection{Magnetron Sputtering}

All thin films and contact electrodes studied in this thesis were deposited using high vacuum magnetron sputtering set-up from AJA 8-gun sputter system [1]. The system utilized for sputtering is shown in Figure 2-1. Sputtering is a physical vapour deposition (PVD) process which employs high voltages to ionize inert gases such as $\mathrm{Ar}, \mathrm{N}_{2}$, and generate plasma [2-5]. The positively charged ions are then accelerated towards a target which forms the cathode and is biased negatively. Figure 2-2 shows the schematic of the sputtering process. The ions are able to dislodge and eject the atoms from the target in a typical line of sight cosine distribution, which then condense on the surfaces that are placed in proximity to the magnetron sputtering cathode. Conductors or metallic targets can be deposited using DC power supply while insulators are deposited using RF power supply. Magnetron sputtering utilizes closed loop 


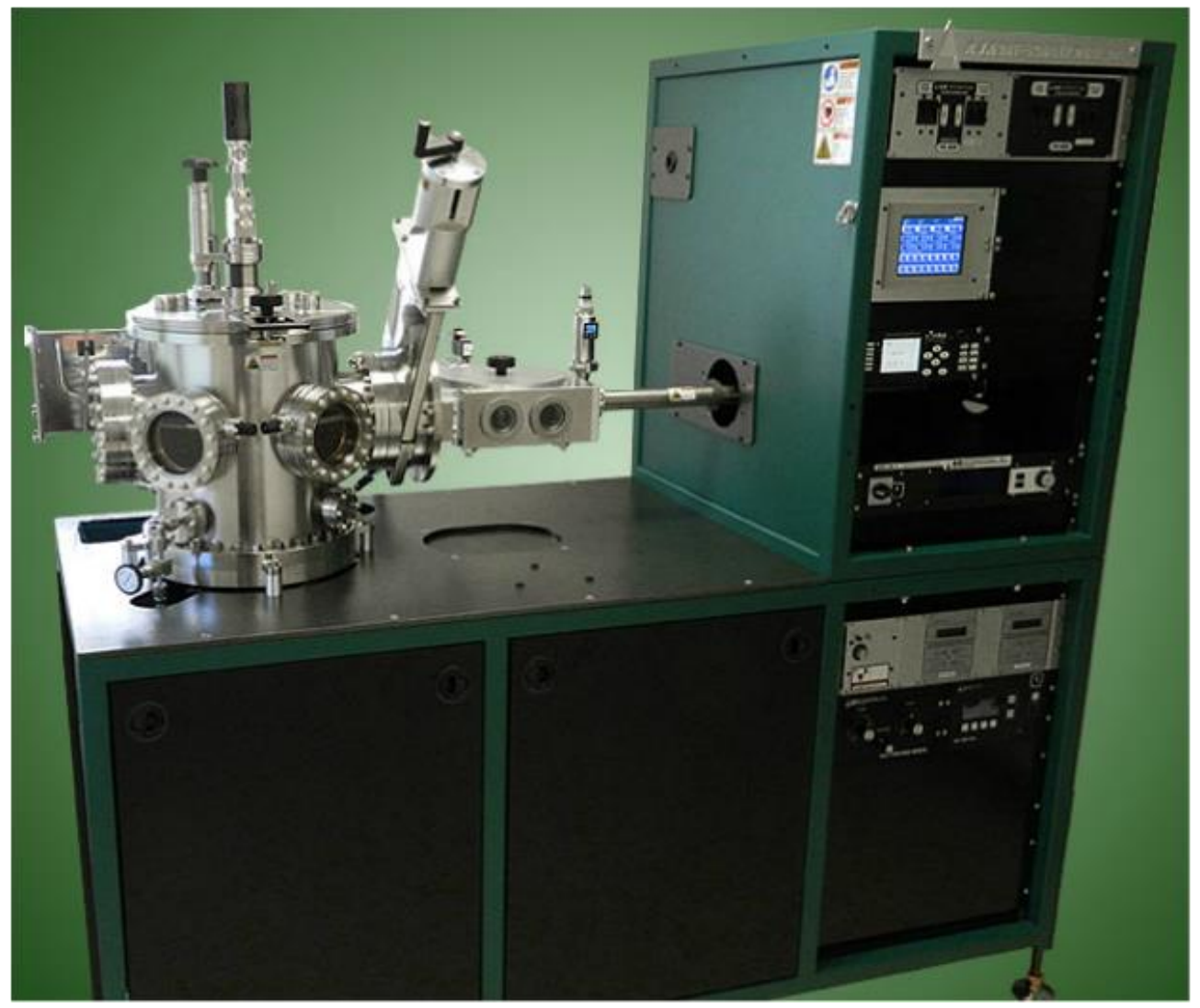

Figure 2-1 Sputter deposition system from AJA [1] used for magnetron sputtering.

magnetic fields around the targets to confine and trap electrons, which increases the path length of electrons causing them to move in spiral motion [6-8]. This enhances the efficiency of the initial ionization process and also allows the plasma to be generated at lower pressure which reduces both background gas incorporation in the growing film and energy losses in the sputtered atoms through collisions.

In the system, a turbomolecular pump can attain background pressure of $\sim 10^{-6}$ Torr. To achieve base pressures of $\sim 10^{-8}$ Torr a cryogenic pump is also employed in conjunction. Such ultra-high vacuum ensures little contamination of the process gases and enhances the mean free paths of the ions to sustain plasma. The working pressure is kept as low as 2-3 mTorr and maintained by flowing Ar at a flow rate of around $20 \mathrm{sccm}$. The sputter yield which corresponds to the number of atoms ejected from the target can be enhanced by increasing the sputter 


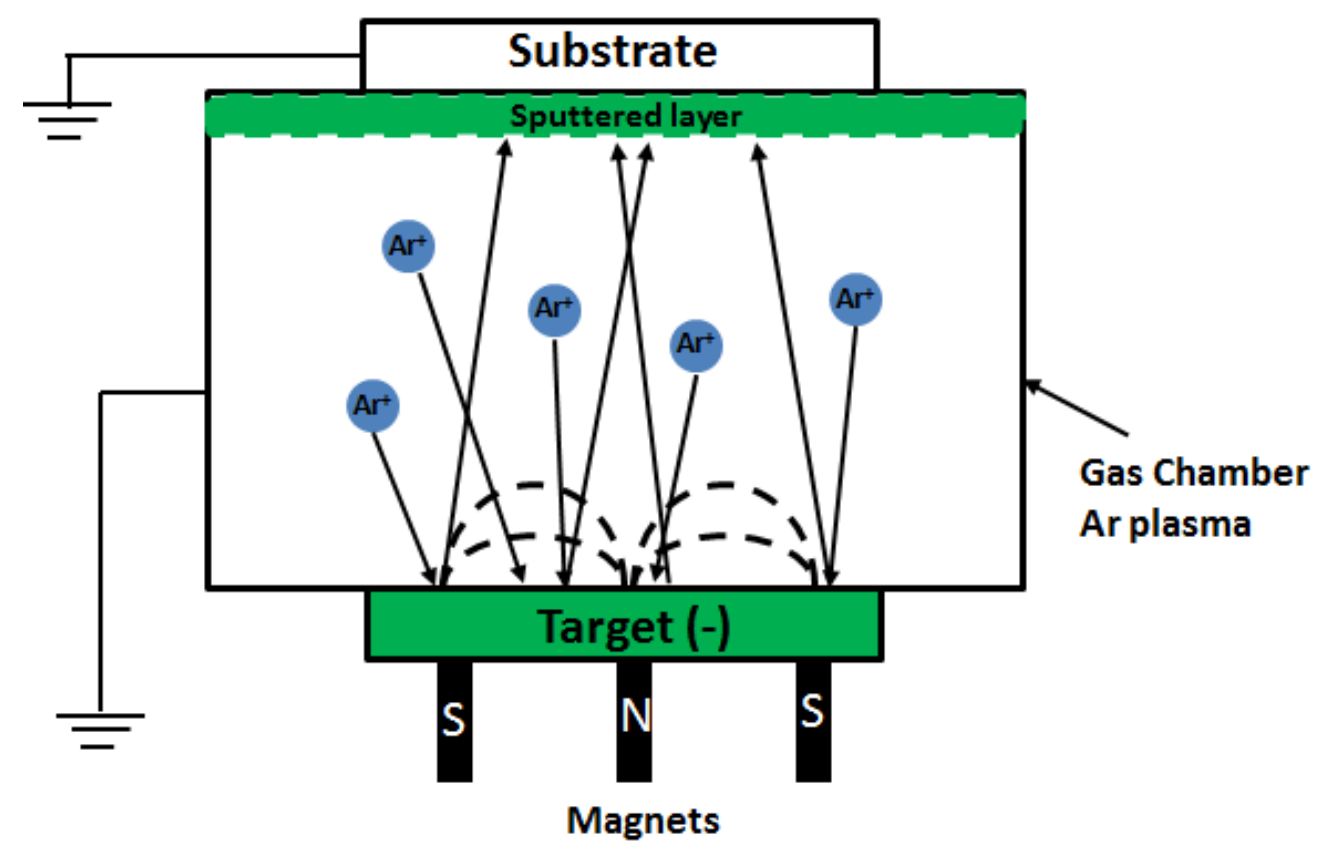

Figure 2-2 Schematic of the magnetron sputtering process. Target is biased negatively and the magnets confine the electrons near the target. The sputtered atoms from target are deposited on the substrate.

power. The deposition rate increases in proportion to the sputtering power. For the present study, the power was fixed at $50 \mathrm{~W}$ and the sputter rate was ascertained by noting the time of deposition and thickness calibrated using X-ray reflectometry (XRR).

\subsection{Device Fabrication}

\subsubsection{Sample preparation}

The substrate employed for thin film deposition and subsequent device patterning is thermally oxidized silicon wafer with thickness of $200-300 \mathrm{~nm}$. The wafers were diced into $1 \times 1 \mathrm{~cm}^{2}$ pieces for patterning a number of devices per diem. The diced wafers undergo substrate cleaning process as described below. 
The substrates are ultrasonicated with acetone for 15 mins to remove the inorganic contaminants. Then they are ultrasonicated with iso-propyl alcohol (IPA) for 15 mins to remove the acetone completely and other contaminants. De-ionized water is used to wash away the IPA contaminants. Subsequently the substrates are blown dry with $\mathrm{N}_{2}$. After cleaning, the substrates were deposited with thin film using magnetron sputtering technique and subsequently patterned using top-down lithography approach with the assistance of EBL and Ar-ion milling for pattern transfer.

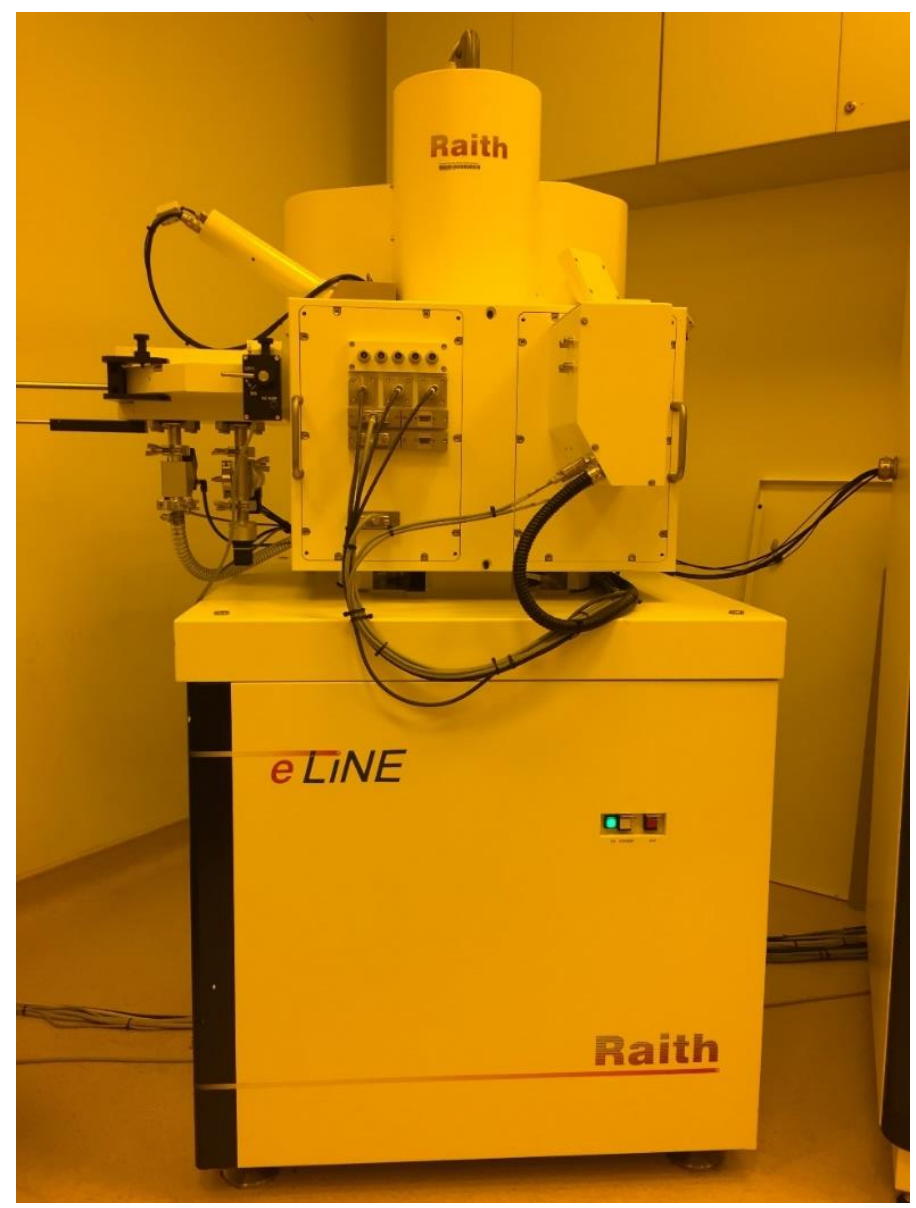

Figure 2-3 Raith e-Line system used for electron beam lithography. 


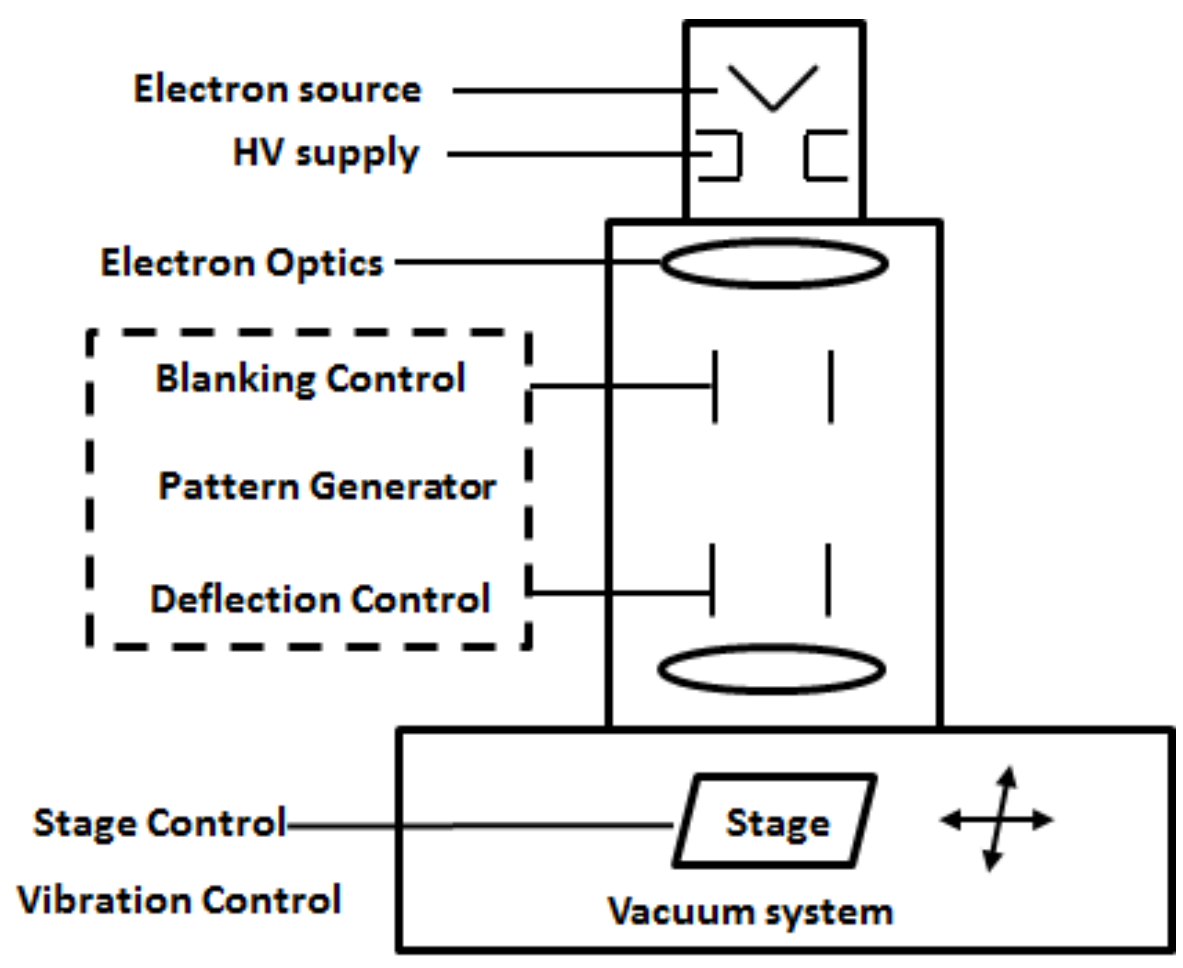

Figure 2-4 Schematic of electron beam lithography process. The electrons arriving from the source are collimated using electromagnetic lenses and passed through the pattern generator before exposing the sample mounted on the stage. Adapted from [9]

\subsubsection{Electron Beam Lithography}

Electron beam lithography (EBL) is a useful tool for creating patterns at nanoscale dimensions [10-12]. The process of lithography involves coating the substrate or the film with a photoresist which is sensitive to energetic electron beams. The substrate is then exposed to a beam of electrons emitted from a source and passed through a pattern generator. The photoresist is an organic compound which becomes either more soluble on exposure (positive resist) or less soluble (negative resist). The photoresist is then developed in a chemical which dissolves the soluble part. Due to low wavelength of electrons, the diffraction is limited and ultra-high resolution in the sub-nm regime can be achieved. 
The system employed for the study in this thesis is Raith e-Line system which is shown in Figure 2-3. The source of electrons is thermionic emission from lanthanum hexaboride. The electrons are accelerated to high voltages and are collimated using electromagnetic lenses. The above features are same as that of a scanning electron microscope (SEM) to which beam blanker and pattern generator are added in an EBL system to control the areas to be exposed. The sample to be exposed is placed on an ultra-high precision stage whose motion is monitored using a laser interferometer. Figure 2-4 shows a schematic of the column of an electron beam exposure system. The lithography is performed using two different techniques: the top-down approach using negative photoresist and the bottom-up approach using positive photoresist. The details of these processes are discussed in the following sections.

\subsubsection{Top-down lithography}

The top-down lithography is accomplished by using a negative photoresist and etching the unexposed regions by dry etching method. The process adopted for top-down lithography is as follows. First the magnetic material to be patterned is deposited on a substrate using the technique of magnetron sputtering. The film is then spin coated with a negative resist such as ma-N 2403 [13], at 6000 revolutions per minute for $60 \mathrm{~s}$. The thickness of the resist obtained is $180-200 \mathrm{~nm}$. The substrate with the coated photoresist is then baked on a hot plate at $100^{\circ} \mathrm{C}$ for 1 minute. The film with the resist is then exposed with electron beams using EBL. The dosage used is $130 \mu \mathrm{C} / \mathrm{cm}^{2}$, with the beam current as $200 \mathrm{pC}$. The aperture and beam voltage are kept as $30 \mu \mathrm{m}$ and $10 \mathrm{keV}$ respectively. The exposed regions become less soluble due to cross-linking of the photoresist. The un-exposed regions are dissolved away by using a developer solution ma-D 525 [14]. The exposed substrate is rinsed in the developer for $90 \mathrm{~s}$ and then in de-ionized water for 2 minutes to wash away the resist. To etch the un-exposed metallic 


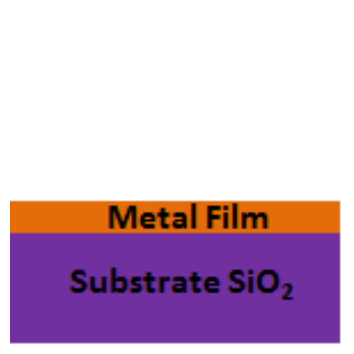

(a)

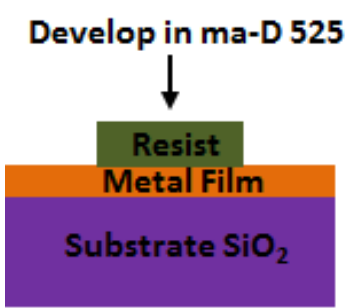

(d)

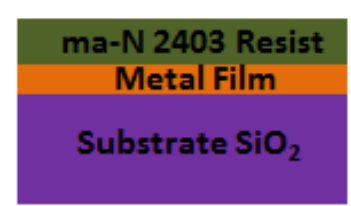

(b)

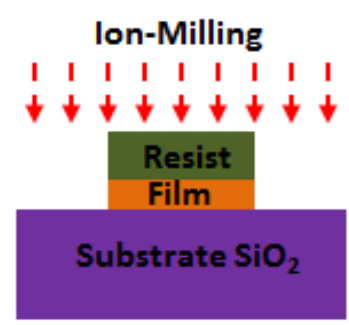

(e)
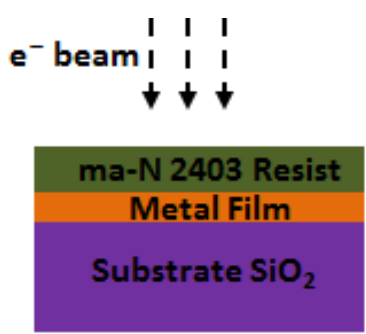

(c)

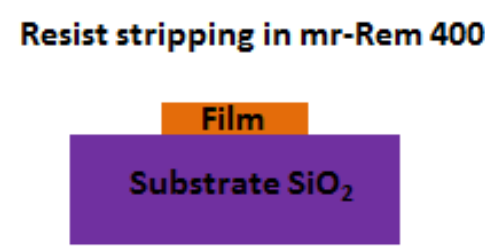

(f)

Figure 2-5 Schematic of top-down lithography process (a) Thin film deposition (b) Negative resist spin-coating (c) Electron beam exposure (d) Develop in chemical to remove un-exposed resist (e) Ion-milling to etch the exposed metal film (f) Resist stripping in chemical solvent.

film and transfer the pattern, the substrate with the film and resist is subjected to ion beam milling which is explained in detail in section 2.2.3. The ion milling which uses Ar atoms to bombard the surface of the sample, etches away the film. Since the resist is much thicker than the metallic film, the resist acts as a hard mask and protects the pattern underneath. Thus the pattern is obtained with resist on top. The resist is then stripped away in a chemical solution such as mr-Rem 400 [15]. The chemical bath with the resist and solution is heated at $85^{\circ} \mathrm{C}$ for 1 hour and then ultrasonicated in the bath for 2 hours. Finally the patterned sample is washed in IPA to remove the chemical. The lithography process schematic is illustrated in Figure 2-5. 


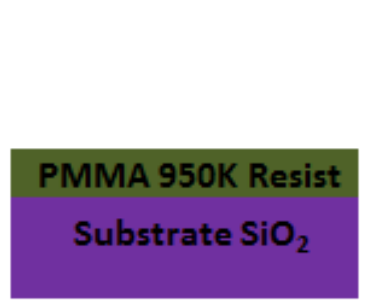

(a)

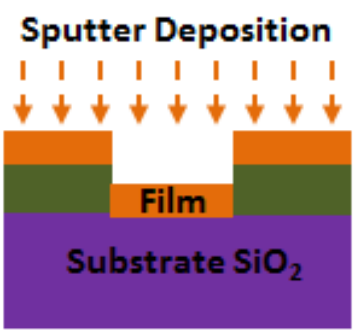

(d)

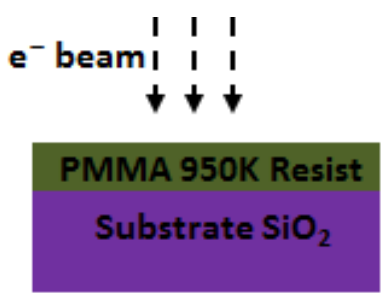

(b)

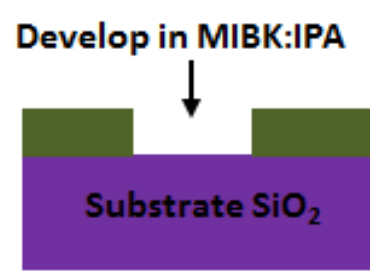

(c)

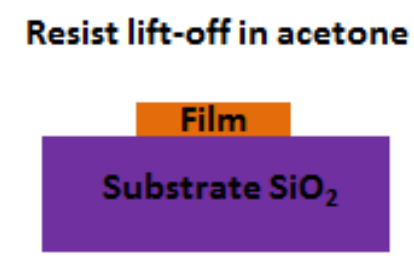

(e)

Figure 2-6 Schematic of bottom-up lithography process (a) Positive resist spin-coating (b) Electron beam exposure (c) Develop in chemical to remove exposed resist (d) Thin film deposition in the exposed patterns (e) Resist lift-off in acetone.

\subsubsection{Bottom-up lithography}

The bottom-up lithography is accomplished by using a positive photoresist and then a lift-off technique after deposition of the film in the patterned structures. This technique was employed for contact formation in the second lithography step. First, the sample to be patterned is spin coated with PMMA 950K [16] positive photoresist at 4000 revolutions per minute for $45 \mathrm{~s}$. The thickness of the resist obtained is $200 \mathrm{~nm}$. Then the sample with the photoresist is baked on a hot plate at $180^{\circ} \mathrm{C}$ for 5 minutes. Now, the sample is exposed to the electron beam using EBL to write the desired pattern. The dosages and EBL settings are similar to that of the topdown approach discussed in the previous section. The exposed regions become more soluble. After exposure, the sample is developed in a 1:3 solution of methyl isobutyl ketone (MIBK) diluted with IPA. The sample is rinsed with MIBK:IPA for 30s and then with IPA alone for another 30s. The exposed regions are dissolved away in the solution. The patterned structure 
is deposited with the required thin film. For contact formation the thin film stack is $\mathrm{Ta}$ (5 $\mathrm{nm}) / \mathrm{Cu}(100 \mathrm{~nm}) / \mathrm{Au}(3 \mathrm{~nm})$. The gold capping is used to prevent oxidation of the copper contacts. Before the deposition, a reverse sputtering using RF bias is employed for 60s at 30 W RF power to remove any oxides or impurities. After the deposition the resist is lift-off by acetone ultrasonication followed by IPA rinse. The lithography process steps are illustrated in Figure 2-6.

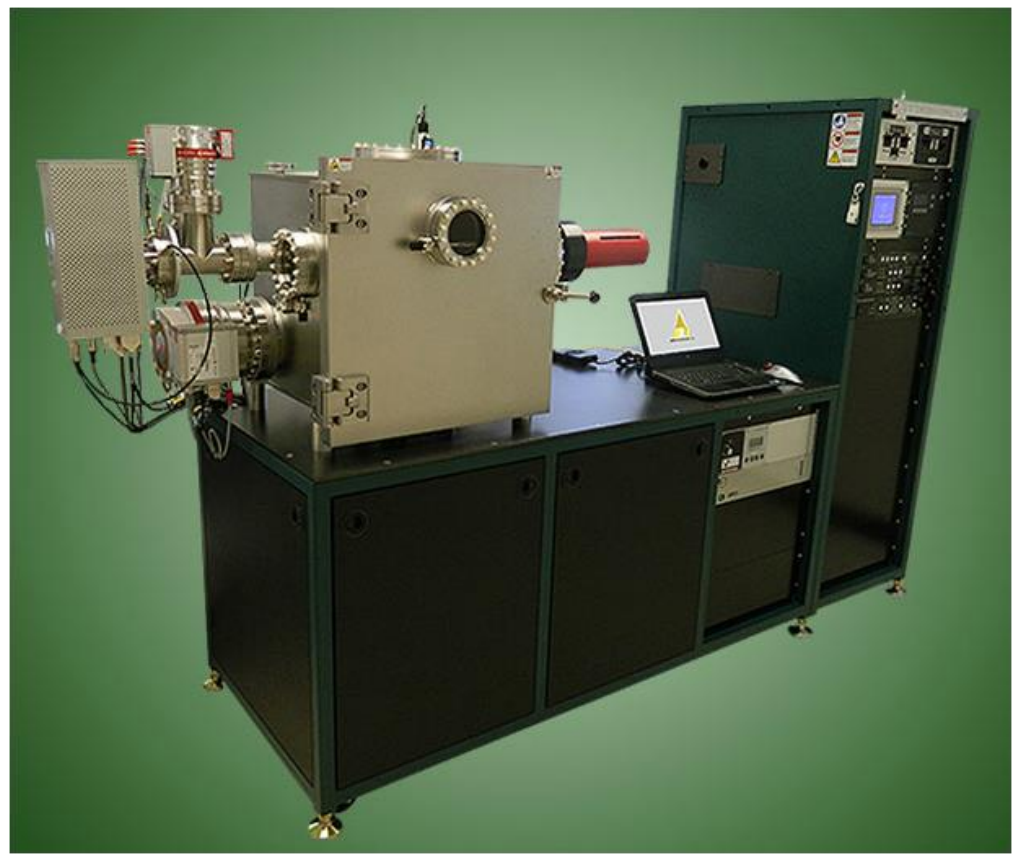

Figure 2-7 Ion-milling system from AJA [17].

\subsubsection{Ion-Milling}

Ion-milling is a dry etching technique where inert gas atoms are bombarded at the surface of the substrate which is placed at a certain angle to the incoming ions $[18,19]$. The ion-milling system used in this thesis is AJA ATC-2020-IM [17] shown in Figure 2-7. The system has a vacuum chamber with a rotatable stage. A turbomolecular pump can reach pressures as low as $10^{-8}$ Torr. The inert gas used is Ar, and a high voltage source is used to generate Ar plasma. 
The Ar ions are accelerated by an optically aligned grid to form highly collimated beam. A neutralizer, which is a tungsten rod for electron emission, is attached just after the source of ions to neutralize the ions and prevent charging of the substrate. The angle of the stage can be adjusted manually and is kept $5^{\circ}$ to the normal i.e. $85^{\circ}$ from the horizontal line of sight of the incoming ions which ensures that re-deposition is minimum. The stage is rotated at 10 revolutions per minute to ensure uniform etching. An Ar flow rate of $14 \mathrm{sccm}$ is maintained and power of etching is kept at $85 \mathrm{~W}$. The ion-milling system is equipped with secondary-ion mass detector (SIMS) which is used to detect elemental, isotopic or molecular composition of the sample surface. The ion-milling technique is primarily employed to etch the metal film during pattern transfer of the top-down lithography process.

\subsection{Magnetic Characterization Techniques}

\subsubsection{Magnetic Force Microscopy}

Magnetic force microscopy (MFM) technique is a variation of atomic force microscopy (AFM) where a tip coated with magnetic material scans a magnetic sample and the interactions of the tip with the stray field of the sample are used to reconstruct the magnetic structure or domains in the sample [20-23]. The MFM signal is often masked by the topography obtained using AFM, hence MFM is performed in the lift-mode where tip is at a certain height above the sample $(10-200 \mathrm{~nm})$. Since magnetostatic forces are long range forces, as compared to atomic forces which are short range, they allow the MFM tip to work at a larger gap between tip and the sample. For a distance $\mathrm{Z}$ of separation between the tip and the sample surfaces, which is less than the tip radius, $R$, the van der Waals forces can be approximated as, $F=-A R / 6 Z^{2}$, where $\mathrm{A}$ is a constant of the order of $5 \times 10^{-20} \mathrm{~J}$ [24]. For sub-micron tips, the van der Waals forces can be neglected compared to the magnetic forces. The MFM imaging in this thesis was performed using Dimension V from Bruker. The principle of operation is as follows: 
A cantilever vibrating at a certain frequency is scanned across the sample surface and a photodetector monitors the rms value of the amplitude of a laser beam reflected by the cantilever. Modifications to the force gradient along the vibration direction (z-direction) shifts the resonance frequency which causes the amplitude to slide up or down the slope of the resonance curve [25]. In the lift mode, the first scan in the close proximity to the surface gives topographical information, while the feedback loop is closed maintaining the amplitude at a certain value. For the second scan, the loop is opened and the scanner repeats the z-movement of the first scan with an offset in the height added. This eliminates the topography. Figure 2-8 shows a schematic of the imaging process. The relative amplitude change at the steepest portion of the resonance curve is given by [26]:

$$
\frac{\Delta \boldsymbol{A}}{A_{0}}=\frac{2 Q}{3 \sqrt{k}} \frac{d \boldsymbol{F}_{n}}{d n} .
$$

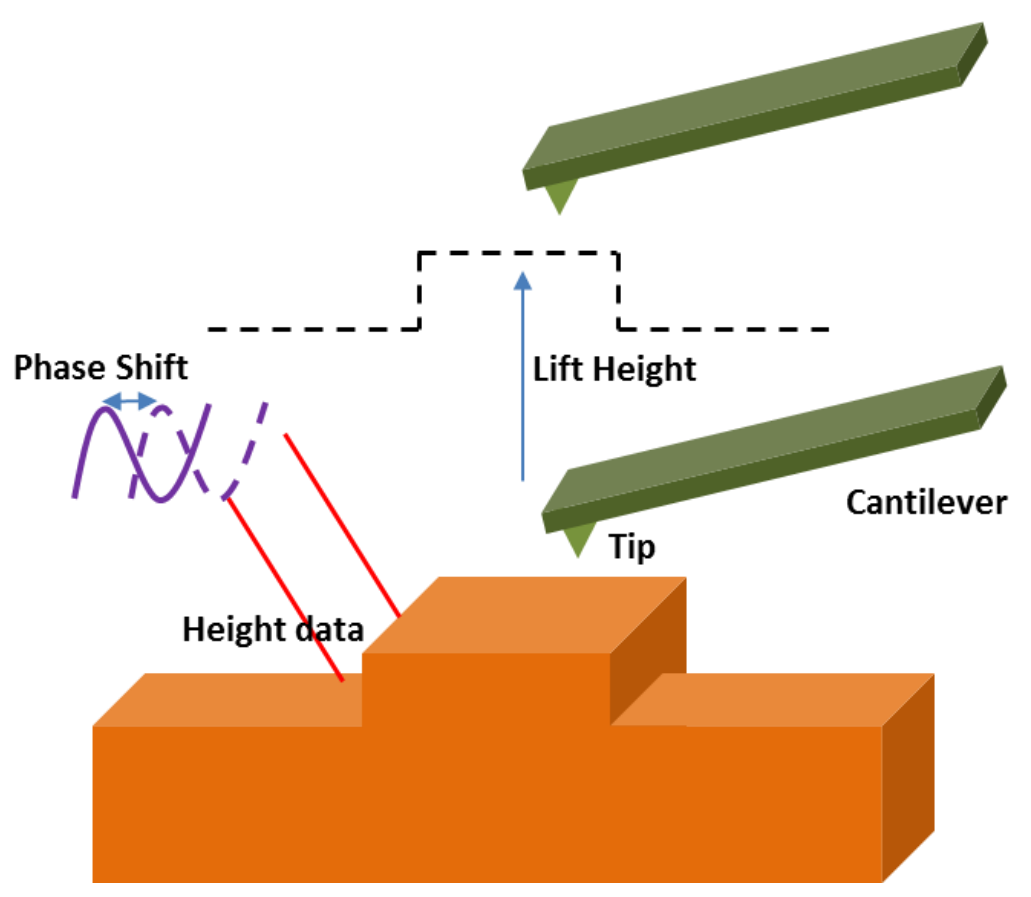

Figure 2-8 Schematic of magnetic force microscopy imaging technique. The lift mode eliminates the topography information. The phase shift induced on account of magnetic force gradient gives information about the domains and magnetic structures. 
where, $A_{0}$ is the amplitude at the resonance frequency, $k$ is the spring constant of the cantilever, $d \boldsymbol{F}_{n} / d n$ is the derivative of the force normal to the cantilever and Q is the quality factor of the resonance. The force gradient acting on the tip can be expressed as:

$$
\frac{d \boldsymbol{F}_{n}}{d n}=\int\left(\boldsymbol{m}_{\boldsymbol{x}} \frac{\partial^{2} H_{x}}{\partial z^{2}}+\boldsymbol{m}_{\boldsymbol{y}} \frac{\partial^{2} H_{y}}{\partial z^{2}}+\boldsymbol{m}_{z} \frac{\partial^{2} H_{z}}{\partial z^{2}}\right) d V
$$

where, $\boldsymbol{m}_{x}, \boldsymbol{m}_{\boldsymbol{y}}, \boldsymbol{m}_{z}, H_{x}, H_{y}, H_{z}$ are the magnetization and field components at location $\mathrm{r}$ within the tip, respectively. The force gradient which causes a shift in the frequency is manifested as a phase difference which is measured in the MFM technique to obtain the magnetic information of the sample.

The limitation with MFM technique is that it cannot directly probe the magnetization, rather the dipole fields generated by magnetization inhomogeneity. For materials with in-plane magnetic anisotropy, these dipole fields are related to the gradient of the magnetization. For perpendicular magnetic anisotropy (PMA) media, the tip sample interaction can be strong enough to change the magnetization of the sample. This is particularly disadvantageous when studying domain wall (DW) dynamics, since the tip can drag the DW as the depinning field of DW is only about 80-100 Oe. Thus in PMA nanowires, technique of magneto-optical Kerr imaging has been utilized to observe DW motion.

\subsubsection{Alternating Gradient Force Magnetometry}

Alternating gradient force magnetometry (AGFM) technique is a quick and useful method for measuring magnetic properties with very high sensitivity down to $10 \mathrm{nemu}$ [27]. The principle lies in sensing the force due to a magnetic field gradient. The sample is mounted on the end of a cantilevered rod incorporated with a piezoelectric element. The sample is first magnetized by a DC magnetic field and is simultaneously subjected to a varying AC field. This alternating field gradient exerts an alternating force on the sample which is proportional to the magnitude 
of the field gradient and the magnetic moment of the sample. This results in the vibration of the sample which is detected by the deflection of the cantilever rod. The output signal can be greatly amplified if operated near the resonance frequency of the cantilever. The operating frequency is tuned between $10-100 \mathrm{~Hz}$ with quality factor, Q, in range of $25-250$. The force due to a magnetic field gradient, $\mathrm{F}_{\mathrm{Z}}$, can be expressed as follows:

$$
\boldsymbol{F}_{Z}=\boldsymbol{m}_{Z}\left(B_{Z}\right) \frac{\partial b_{Z}}{\partial Z}
$$

where, $\boldsymbol{m}$ is the total magnetic moment, B is the magnetizing field and $\mathrm{b}$ is the gradient field. The AGFM used for the current study is PMC MicroMag 2900. Figure 2-9 shows a schematic of the AGFM set-up. Samples are diced to sizes $3 \mathrm{~mm} \times 3 \mathrm{~mm}$ with a known thickness of the magnetic layer. The magnetic moment obtained is normalized with the volume to obtain the magnetization in the units of emu/cc. There are separate probes for perpendicular and in-plane magnetic field directions. The system is calibrated by mounting a calibration sample of known magnetic moment. The magnetic moment of the silicon dioxide wafer which is the substrate for our samples is measured to incorporate diamagnetic correction in the M-H loops.

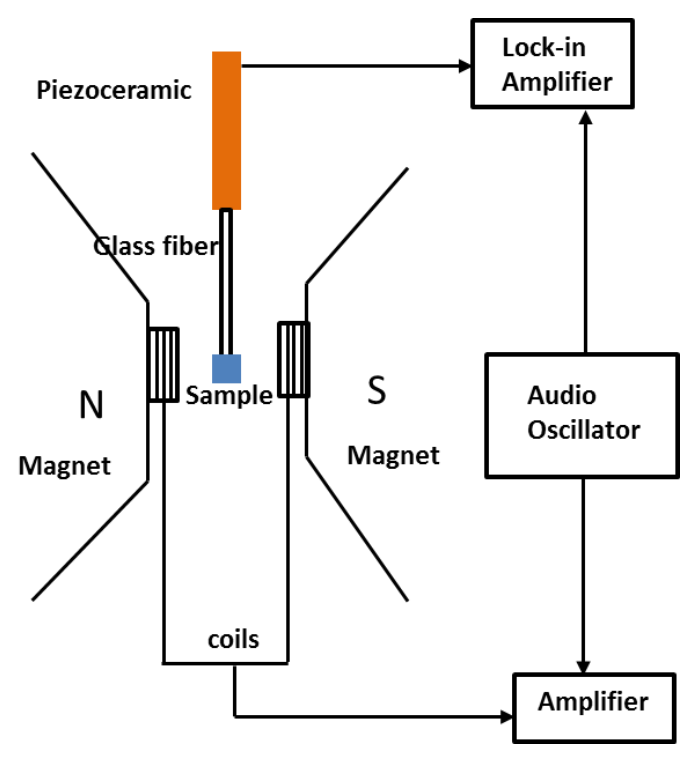

Figure 2-9 Schematic of alternate gradient force magnetometry. The magnetic force gradient due to interaction of magnetic moment of the sample with the applied alternating field deflects the sample and the piezoceramic holder. The deflection gives information of the magnetic moment of the sample. 


\subsubsection{Magneto-Optical Kerr Effect}

Magneto-optical Kerr effect (MOKE) polarimetry provides an alternate method for obtaining magnetic hysteresis curves, even though they do not provide absolute values of the magnetic moments. The Kerr microscopy technique based on the Kerr effect $[28,29]$ is an effective tool for direct observation of DW and magnetization in the devices [30-33]. A linearly polarized laser light illuminates the test sample. The magnetization of the sample interacts with the polarized light beam and the light reflected from the sample is rotated in polarization with respect to the incident light. This light is passed through an analyser which is nearly crosspolarized with the incident polarizer. The photodetector measures the incident intensity and a lock-in amplifier reads the corresponding voltage signal proportional to the incident intensity which is in- turn a measure of the magnetization of the sample. The reason behind the change in polarization of the plane polarized light is briefly discussed as follows.

Light incident on the sample is referred to as p-polarized if the electric field is polarized in the plane of incidence and is s-polarized if the electric field is polarized perpendicular to the plane of incidence. When p-polarized light is reflected off a magnetic surface, the reflected light along with the p-component also has some s-component. In general the second electric field component is out-of-phase with the reflected p-component which makes the reflected light elliptically polarized. The major axis also gets rotated from the initial polarized plane. Similar is the case when an s-polarized light is reflected off a magnetic surface. This is referred to as Kerr ellipticity and Kerr rotation. The plane polarized light can also be considered as composed of two circularly polarized components $\mathrm{L}$ and $\mathrm{R}$. These two components travel with different velocities in the magnetized medium since they experience different refractive indices. Upon reflection the two modes recombine to give Kerr ellipticity and rotation. A change in polarization of the in-phase component of the reflected light gives rise to rotation and a change in polarization of the out-of-phase component gives rise to ellipticity. 

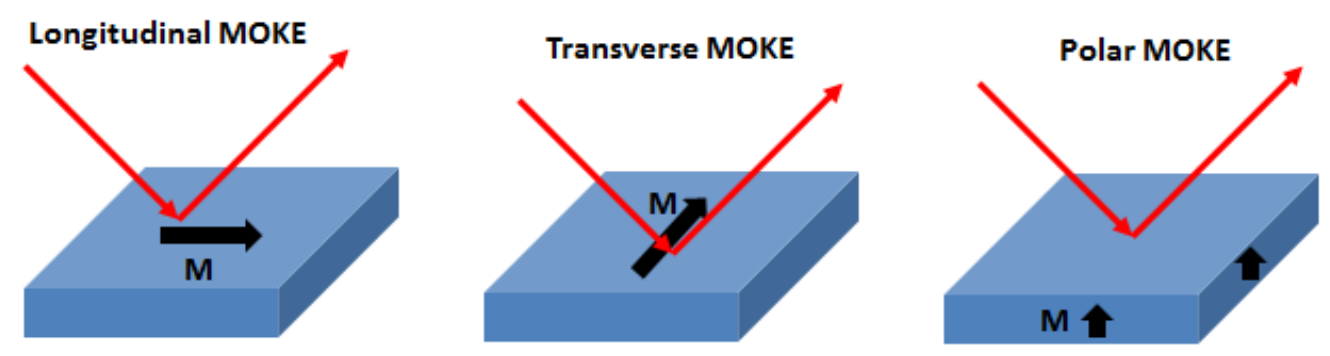

Figure 2-10 Geometries of magneto-optical Kerr effect measurement. The longitudinal and polar MOKE modify the polarization of the incident light whereas the transverse MOKE affects the intensity.

In simplified terms, three types of Kerr effect exist as shown in Figure 2-10. In the longitudinal Kerr effect, the magnetization is in the plane of the sample and is parallel to the plane of incidence of the light. In the transverse Kerr effect also the magnetization is in the plane of the sample, however, it is perpendicular to the plane of light incidence. In the polar Kerr effect, magnetization is perpendicular to the sample plane and is parallel to the plane of incidence of light. The longitudinal and polar effects generally alter the polarization of light from plane to elliptical and cause Kerr rotation. But, the transverse effect does not change the plane of polarization. This is due to the fact, that transverse effect does not have Lorentz force component (s-polarized) or the induced component (p-polarized) has the same polarization as the incident polarization.

Kerr microscopy is a technique based on the Kerr effect and gives a visual evidence of magnetization behaviour of the test device. For the PMA samples under study, polar Kerr has been employed to detect the presence of DW. Figure 2-11 shows the schematic of the Kerr microscopy technique. Halogen lamp is used as the light source. The light is plane polarized by passing it through a polarizer. This polarized light falls on the magnetized sample which has already been injected with a DW. The sample induces Kerr rotation and this reflected light is passed through another polarizer which acts as an analyzer. The reflected light is captured using 
a camera and the image is processed to obtain the final Kerr image. The Kerr setup employed in this study uses a microscope provided with two polarizers for polarizing the incident light and capturing the reflected light respectively.

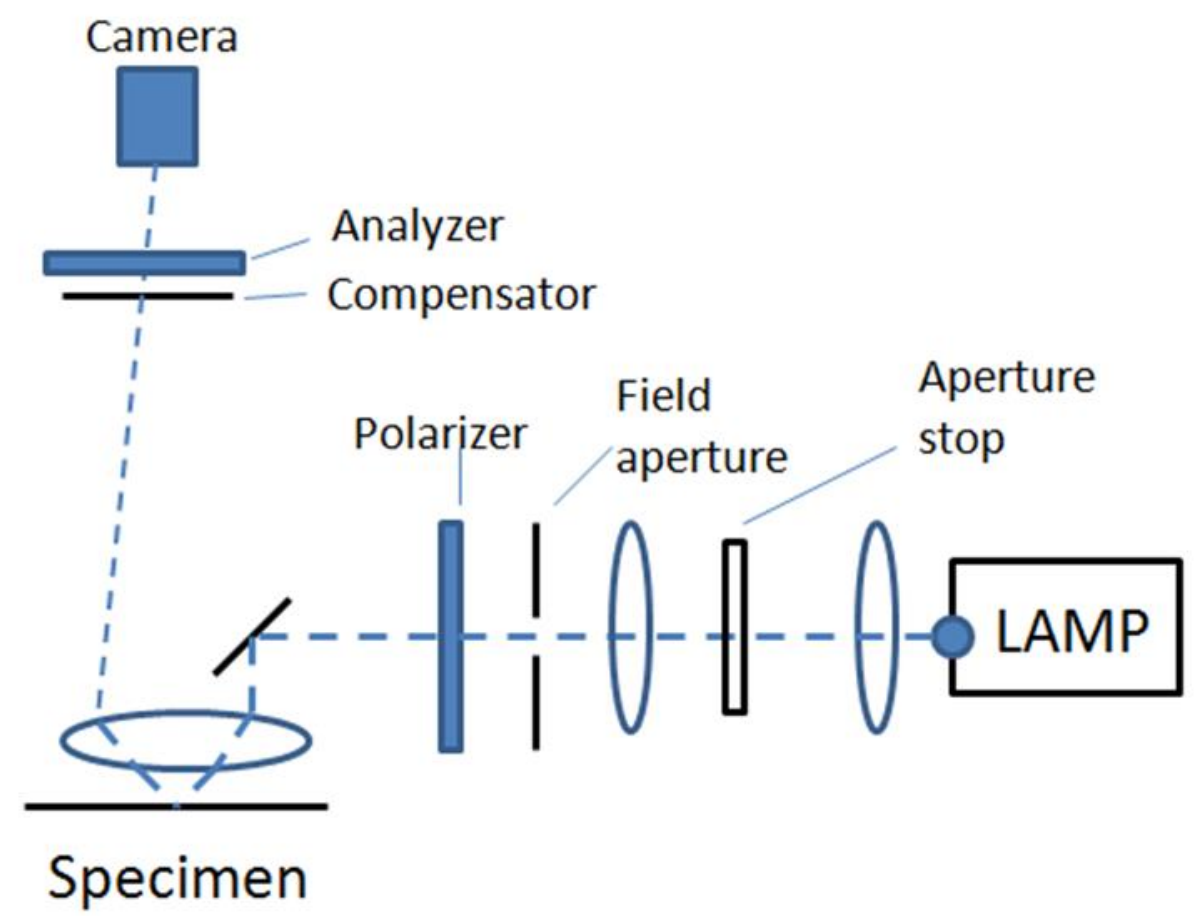

Figure 2-11 Schematic of Kerr microscopy set-up.

\subsection{Electrical Characterization Techniques}

The electrical characterizations for the work presented in this theses were carried out using Cascade Microtech RF probe station, shown in Figure 2-12 (a), equipped with pico-second pulse generator from Pulse Labs (Picosecond 10300B), shown in Figure 2-12 (b). The probe station is equipped with 6 DC probes and 2 ground-source-ground (GSG) probes for handling high frequency signals. The AC and DC current sources and voltmeter were sourced from Keithley 2400 series. A $6 \mathrm{GHz}$ oscilloscope from Teledyne Lecroy WaveRunner series was employed for signal detection. The out-of-plane and in-plane magnetic fields were applied 
using in-house electromagnets built to provide maximum fields of $4 \mathrm{kOe}$ and $600 \mathrm{Oe}$ respectively.

(a)

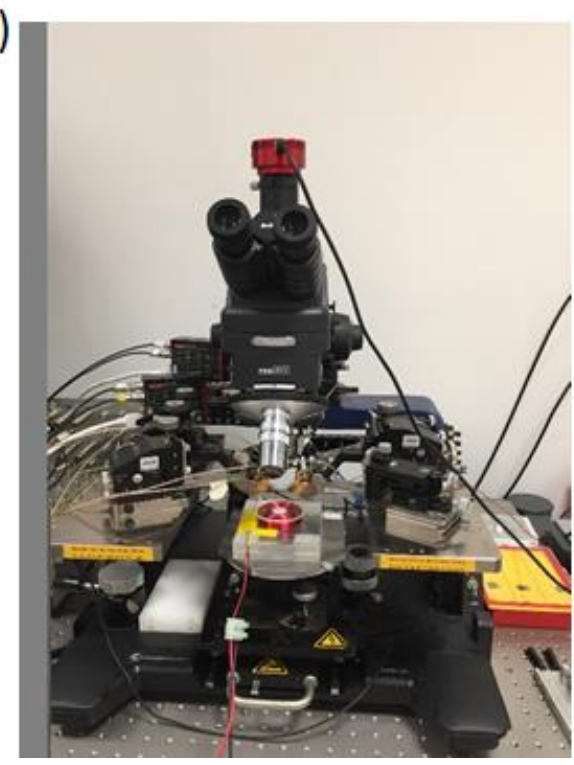

(b)

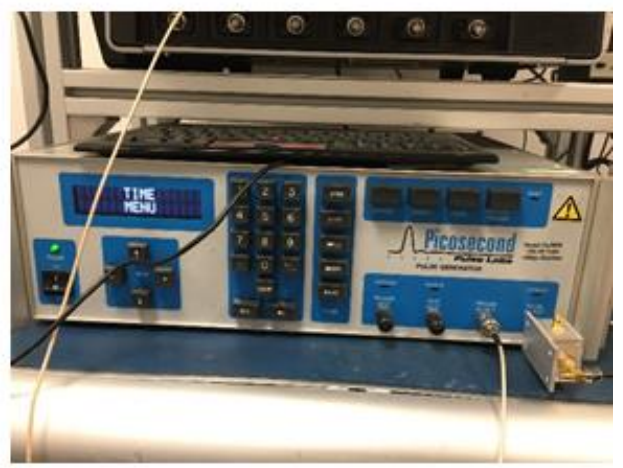

Figure 2-12 (a) Cascade Microtech RF probe station for electrically probing the devices (b) Pico-second pulse generator set-up.

\subsubsection{Domain wall nucleation, detection and driving procedure}

Figure 2-13 shows the scanning electron microscopy (SEM) image of a $300 \mathrm{~nm}$ wide PMA Hall cross structure along with the schematic of the measurement set-up. The PMA nanowire is initially saturated with an out-of-plane magnetic field along the $+z$-direction. The strip-line at electrode $\mathrm{A}$ formed of $\mathrm{Ta} / \mathrm{Cu}$ is used for nucleation of the $\mathrm{DW}$. To nucleate a DW, switch $\mathrm{S} 1$ is closed and switch S2 is open. An electric pulse of voltage 6V (100 mA current) and pulse width $50 \mathrm{~ns}$ is applied to the strip-line at electrode A. The AC flows through the top part of the bias-tee through the strip-line and sinks into the ground via oscilloscope. The current generates a localized Oersted field and a DW is nucleated in the vicinity of the strip-line. 


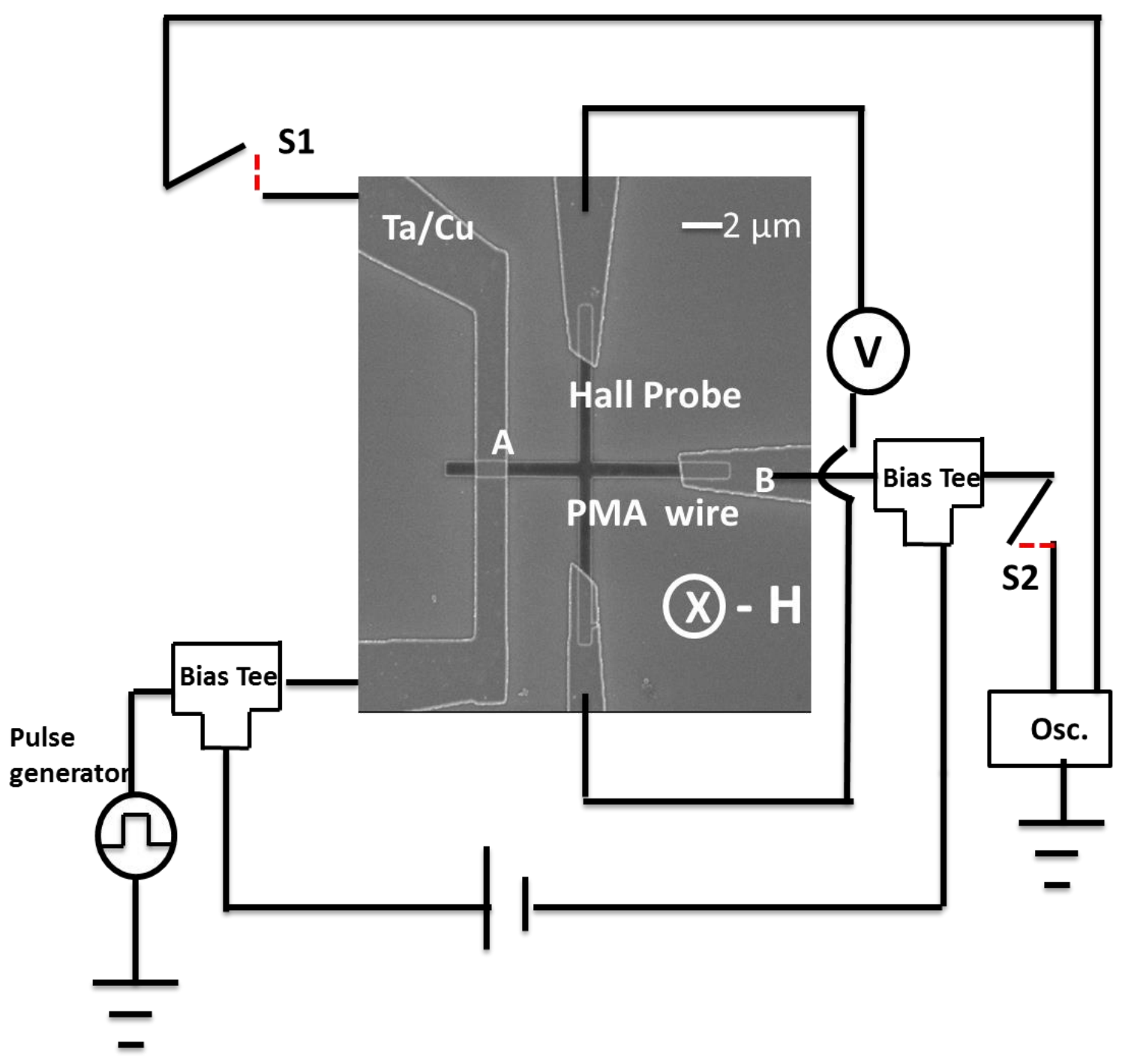

Figure 2-13 Measurement set-up to electrically nucleate, detect and drive the domain wall. For nucleation switch $\mathrm{S} 1$ is closed while $\mathrm{S} 2$ is open. For domain wall driving switch $\mathrm{S} 2$ is closed while $\mathrm{S} 1$ is open.

To detect the presence of DW, anisotropic magneto-resistance (AMR) technique can be used [34-36]. The creation of a DW would drop the resistance of the nanowire. However, since the change in resistance is very small $(\sim 0.1 \Omega)$, it can be masked by the electrical noise in the setup. An alternative method for detecting the presence of a DW is based on the principle of anomalous Hall effect $[37,38]$. The change in magnetization of the nanowire from up to down would change the resistance at the Hall probes since the Hall resistance is proportional to the perpendicular component of the magnetization. It is to be noted that contribution due to planar 
Hall effect is small and neglected in this case. To detect the presence of the DW a $100 \mu \mathrm{A}$ DC read current is passed through the nanowire. The magnetic field is swept in the $-z$-direction. This drives the DW towards the Hall probe. A drop in the resistance at the Hall probe signals the presence and propagation of DW towards the Hall probe.

To drive the DW using electric current and the phenomena of spin-transfer torque (STT) [39, 40], switch $\mathrm{S} 2$ is closed while $\mathrm{S} 1$ is open. The negative driving pulse is applied using the pulse generator, the $\mathrm{AC}$ flows from $\mathrm{B}$ to $\mathrm{A}$ in the nanowire and the electron motion is from $\mathrm{A}$ to $\mathrm{B}$ which flows to the ground through the oscilloscope. The DW would propagate from A to B against the direction of the current. After every pulse, a read current of $100 \mu \mathrm{A}$ DC is passed through the nanowire and the Hall voltage is measured. The drop in Hall voltage would signal the propagation of the DW towards the Hall probe. By noting the distance between the stripline and the Hall probe, the DW velocity can be estimated from the known pulse width. This is a time-of-flight method for estimating the DW velocity. 


\section{Bibliography}

[1] Available: http://www.ajaint.com/atc-orion-series-sputtering-systems.html

[2] D. E. Harrison Jr, "Theory of the sputtering process," Physical Review, vol. 102, p. 1473, 1956.

[3] P. Sigmund, "Theory of sputtering. I. Sputtering yield of amorphous and polycrystalline targets," Physical review, vol. 184, p. 383, 1969.

[4] H. Oechsner, "Sputtering - a review of some recent experimental and theoretical aspects," Applied Physics A: Materials Science \& Processing, vol. 8, pp. 185-198, 1975.

[5] R. Behrisch and K. Wittmaack, Sputtering by particle bombardment vol. 1: Springer Berlin, 1981.

[6] P. Kelly and R. Arnell, "Magnetron sputtering: a review of recent developments and applications," Vacuum, vol. 56, pp. 159-172, 2000.

[7] S. S. Parkin, C. Kaiser, A. Panchula, P. M. Rice, B. Hughes, M. Samant, et al., "Giant tunnelling magnetoresistance at room temperature with $\mathrm{MgO}$ (100) tunnel barriers," Nature materials, vol. 3, pp. 862-867, 2004.

[8] Y. Xiang, W. Chengbiao, L. Yang, Y. Deyang, and X. Tingyan, "Recent developments in magnetron sputtering," Plasma Science and Technology, vol. 8, p. 337, 2006.

[9] M. A. Mohammad, M. Muhammad, S. K. Dew, and M. Stepanova, "Fundamentals of Electron Beam Exposure and Development," in Nanofabrication, M. Stepanova and S. K. Dew, Eds., ed New York: Springer, 2011, pp. 11-41.

[10] L. F. Thompson, "An introduction to lithography," ed: ACS Publications, 1983.

[11] A. Broers, A. Hoole, and J. Ryan, "Electron beam lithography-Resolution limits," Microelectronic Engineering, vol. 32, pp. 131-142, 1996.

[12] A. A. Tseng, K. Chen, C. D. Chen, and K. J. Ma, "Electron beam lithography in nanoscale fabrication: recent development," IEEE Transactions on Electronics Packaging Manufacturing, vol. 26, pp. 141-149, 2003.

[13] micro resist technology. Available: http://www.microchem.com/PDFs_MRT/maN\%202400\%20overview.pdf

[14] micro resist technology. [Online]. Available: http://kni.caltech.edu/facilities/msds/liquid/developer-for-photoresist-ma-D-525-MicroResist-Technology-05272008.pdf

[15] micro resist technology. Available: http://www.nanophys.kth.se/nanophys/facilities/nfl/resists/Removers-mr-400-500-2013.pdf

[16] Micro Chem. Available: http://microchem.com/pdf/PMMA Data Sheet.pdf

[17] [Online]. Available: http://www.ajaint.com/ion-milling-systems.html

[18] R. E. Lee, "Microfabrication by ion-beam etching," Journal of Vacuum Science and Technology, vol. 16, pp. 164-170, 1979.

[19] P. G. Glöersen, "Ion- beam etching," Journal of Vacuum Science and Technology, vol. 12, pp. 28-35, 1975.

[20] U. Hartmann, "Magnetic force microscopy," Annual Review of Materials Science, vol. 29, pp. 53-87, 1999.

[21] J. Garcia, A. Thiaville, and J. Miltat, "MFM imaging of nanowires and elongated patterned elements," Journal of magnetism and magnetic materials, vol. 249, pp. 163-169, 2002.

[22] J. Lohau, S. Kirsch, A. Carl, G. Dumpich, and E. Wassermann, "Quantitative determination of effective dipole and monopole moments of magnetic force microscopy tips," Journal of applied physics, vol. 86, pp. 3410-3417, 1999.

[23] A. Hubert, W. Rave, and S. Tomlinson, "Imaging magnetic charges with magnetic force microscopy," Physica Status Solidi B Basic Research, vol. 204, pp. 817-828, 1997.

[24] J. J. Saenz, N. Garcia, and J. C. Slonczewski, "Theory of Magnetic Imaging by Force Microscopy," Applied Physics Letters, vol. 53, pp. 1449-1451, Oct 101988. 
[25] W. Rave, L. Belliard, M. Labrune, A. Thiaville, and J. Miltat, "Magnetic Force Microscopy Analysis of Soft Thin-Film Elements," Ieee Transactions on Magnetics, vol. 30, pp. 4473-4478, Nov 1994.

[26] Y. Martin, C. C. Williams, and H. K. Wickramasinghe, "Atomic Force Microscope Force Mapping and Profiling on a Sub 100-a Scale," Journal of Applied Physics, vol. 61, pp. 47234729, May 151987.

[27] P. J. Flanders, "An Alternating-Gradient Magnetometer," Journal of Applied Physics, vol. 63, pp. 3940-3945, Apr 151988.

[28] P. He, W. A. McGahan, J. A. Woollam, F. Sequeda, T. McDaniel, and H. Do, "Magneto-optical Kerr effect and perpendicular magnetic anisotropy of evaporated and sputtered $\mathrm{Co} / \mathrm{Pt}$ multilayer structures," Journal of applied physics, vol. 69, pp. 4021-4028, 1991.

[29] K. H. J. Buschow, Handbook of magnetic materials vol. 15: Elsevier, 2003.

[30] S. H. Yang, K. S. Ryu, and S. Parkin, "Domain-wall velocities of up to $750 \mathrm{~m} \mathrm{~s}(-1)$ driven by exchange-coupling torque in synthetic antiferromagnets," Nature Nanotechnology, vol. 10, pp. 221-6, Mar 2015.

[31] R. Lavrijsen, D. M. F. Hartmann, A. Van Den Brink, Y. Yin, B. Barcones, R. A. Duine, et al., "Asymmetric magnetic bubble expansion under in-plane field in Pt/Co/Pt: Effect of interface engineering," Physical Review B - Condensed Matter and Materials Physics, vol. 91, 2015.

[32] K. S. Ryu, L. Thomas, S. H. Yang, and S. Parkin, "Chiral spin torque at magnetic domain walls," Nature Nanotechnology, vol. 8, pp. 527-533, Jul 2013.

[33] I. M. Miron, T. Moore, H. Szambolics, L. D. Buda-Prejbeanu, S. Auffret, B. Rodmacq, et al., "Fast current-induced domain-wall motion controlled by the Rashba effect," Nat Mater, vol. 10, pp. 419-23, Jun 2011.

[34] L. Bogart and D. Atkinson, "Domain wall anisotropic magnetoresistance in planar nanowires," Applied physics Letters, vol. 94, p. 042511, 2009.

[35] M. Kläui, C. Vaz, J. Rothman, J. Bland, W. Wernsdorfer, G. Faini, et al., "Domain wall pinning in narrow ferromagnetic ring structures probed by magnetoresistance measurements," Physical review letters, vol. 90, p. 097202, 2003.

[36] M. Hayashi, L. Thomas, Y. B. Bazaliy, C. Rettner, R. Moriya, X. Jiang, et al., "Influence of current on field-driven domain wall motion in permalloy nanowires from time resolved measurements of anisotropic magnetoresistance," Physical review letters, vol. 96, p. 197207, 2006.

[37] M. Yamanouchi, D. Chiba, F. Matsukura, and H. Ohno, "Current-induced domain-wall switching in a ferromagnetic semiconductor structure," Nature, vol. 428, pp. 539-42, Apr 01 2004.

[38] D. Ravelosona, D. Lacour, J. A. Katine, B. D. Terris, and C. Chappert, "Nanometer scale observation of high efficiency thermally assisted current-driven domain wall depinning," Physical Review Letters, vol. 95, Sep 92005.

[39] L. Berger, "Emission of spin waves by a magnetic multilayer traversed by a current," Physical Review B, vol. 54, pp. 9353-9358, Oct 11996.

[40] J. C. Slonczewski, "Current-driven excitation of magnetic multilayers," Journal of Magnetism and Magnetic Materials, vol. 159, pp. L1-L7, Jun 1996. 


\section{Chapter 3}

\section{Controlled Motion of Domain-Wall in Network Nanostructures with In-Plane Magnetization Anisotropy}

Ferromagnetic network structures are an important system to study both from a technological and fundamental standpoint. The ability to control the domain wall (DW) trajectory in such structures would potentially have applications in magnetic logic $[1,2]$; with presence or absence of DWs being equivalent to logic bits and number of branches equivalent to the number of output states. Fundamentally, network structures provide a system to investigate and observe the existence of magnetic monopoles at room temperature in artificial spin-ice lattices [3-7]. In these network structures, the magnetization dynamics is initiated via absorption and emission of DWs at the vertex. The topological charge or defect at the vertex plays a key role in the magnetization reversal process $[8,9]$. The vertex charge can govern the annihilation and emission of a new DW. The DW itself can be characterized by its topological charge which determines its chirality. A vortex DW is a combination of two $-1 / 2$ edge defects (vertices) and +1 bulk defect (core). A transverse DW is composed of $\pm 1 / 2$ edge defects. The total topological charge of the DW is zero and is conserved during DW transformations [8].

Recently, Pushp et al. [9] reported that a vortex DW would follow a trajectory in Y-branch structures which is strongly dependent on its chirality. Ziessler et al. [10] reported non-random path for transverse DWs in an artificial spin-ice lattice, which was attributed to the DW following a chirality dependent path through the network. Thus, deterministic trajectory of DWs through the network structures depends on the robustness and stability of the chirality. At low magnetic field strengths, the chirality of the DW is preserved. However, above a certain field strength, termed as the Walker field, the DW chirality alternates between Bloch and Neel 
as is propagates. This is termed as Walker breakdown [11]. The DW is able to maintain its chirality only till it propagates a characteristic length called the fidelity length. For DWs propagating at larger fields and beyond their fidelity lengths, it is not possible to control their trajectories in a network structure. In such structures, the DW trajectory is expected to be stochastic. Burn et al. [12] showed that the trajectory of a DW propagating in a branch structure alternates between upper and lower branch at larger magnetic field strengths.

This chapter focusses on achieving deterministic trajectory of DWs in network structures and to understand the DW transformations at the bifurcation with the help of topological defects. The first half of the chapter emphasizes on the chirality dependent selective trajectory of transverse DW in a Y-branch structure. The motivation for selecting Y-branch structure is kagome artificial spin-ice lattice which is a hexagonal structure of frustrated magnetic nanobars $[5,13,14]$, and Y-branch offers a simple unit cell and a system to study this structure. The artificial spin-ice are important system for fundamental study as they allow the observation of magnetic monopoles at room temperature $[4,6]$. The ferromagnetic material studied is permalloy, $\mathrm{Ni}_{80} \mathrm{Fe}_{20}$. The micromagnetic simulations show that the $\mathrm{DW}$ undergo complex transformation with re-allocation of edge defect at the bifurcation. In the second half of the chapter, we show that by adding a geometrical asymmetry in the branch structure a constrained and deterministic motion of DW is possible independent of its chirality. The DW is able to propagate a deterministic trajectory in a branch structure over distances longer than its fidelity length. These concepts are applied to design and demonstrate a programmable logic device which is discussed in the next chapter. 


\subsection{Topological defects in ferromagnetic structures}

Ferromagnetic structures can be regarded as ordered systems possessing discrete symmetry and can be theoretically described using the Ising model [15]. Ferromagnetic systems can be described by a function that assigns to every point in the region an order parameter $[15,16]$. The order parameter in a two dimensional film plane can be described by a unit vector of magnetization, such that it is constrained to lie in the plane. Thus, the order parameter can be taken as the circumference of a circle and also termed as $\theta$-space, where $\theta$ is the angle made by the magnetization with respect to the $x$-axis. Figure 3-1 (a) shows the order parameter space. The order parameter is not defined at a singularity in the medium which is termed as a topological defect. In Figure 3-1 (a), the region $\Omega$, encompasses the topological defect or singularity at the origin (shown by a red circle). The topological defect is associated with a winding number, which is the angle, $\theta$, traversed while completing a closed circuit around the singularity or the defect when the magnetization vector is being mapped. Since the order parameter space is a circle, the winding number, $n$, is the number of times the order space is traversed an angle $2 \pi$ while completing a loop around the defect.

Tchernyshyov et al. proposed the concept of utilizing topological defects $n= \pm 1$ and $n=$ $\pm 1 / 2$ for describing the winding numbers or topological defects for DWs [8]. They proposed in analogy to the XY model [15], that the integer winding numbers in the bulk are obtained from solutions of the Laplace equation. The fractional winding numbers, $n= \pm 1 / 2$ can be obtained from method of images in the XY model for electrostatic charges. Consider the case of transverse DWs. A tail-to-tail up (TT-U) DW is shown in Figure 3-1b (i). As DW propagates along the $x$-direction, the spins would rotate in clockwise direction across the DW as shown by the schematic on the right. As $\theta$ is rotated along the anti-clockwise direction the order space is traversed in the clockwise direction by an amount $\pi$, thus the winding number for tip of the triangular region of the DW is $-1 / 2$. Since total winding number is 0 as we complete the full 
(a)

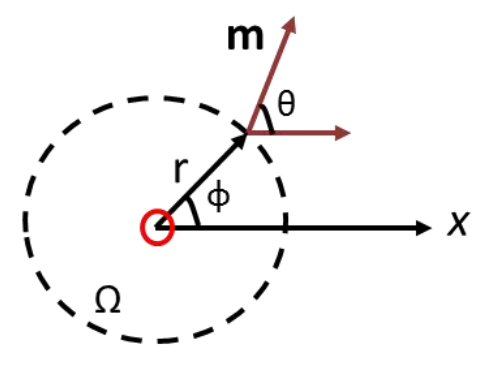

(b)

(i)
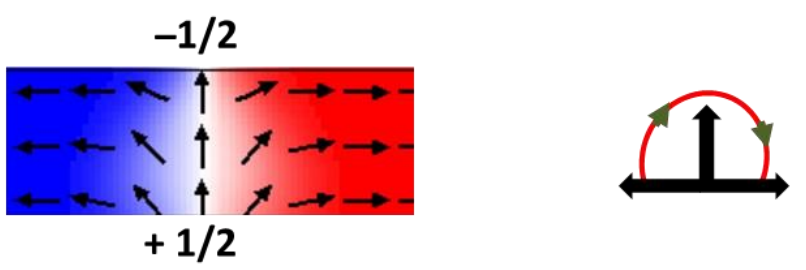

(ii)
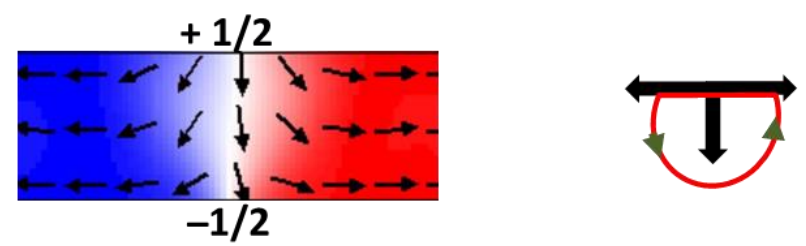

(iii)
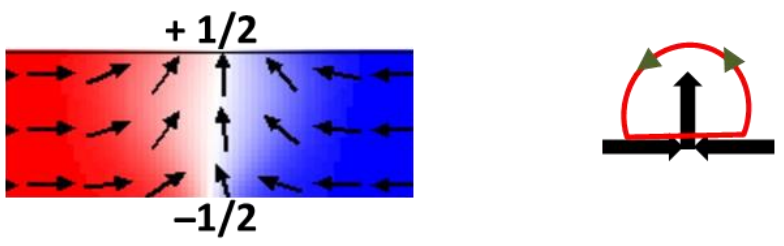

(iv)

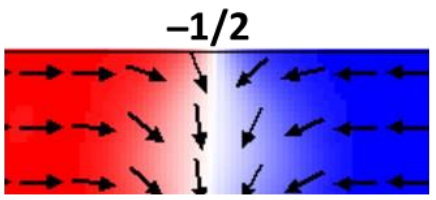

$+1 / 2$
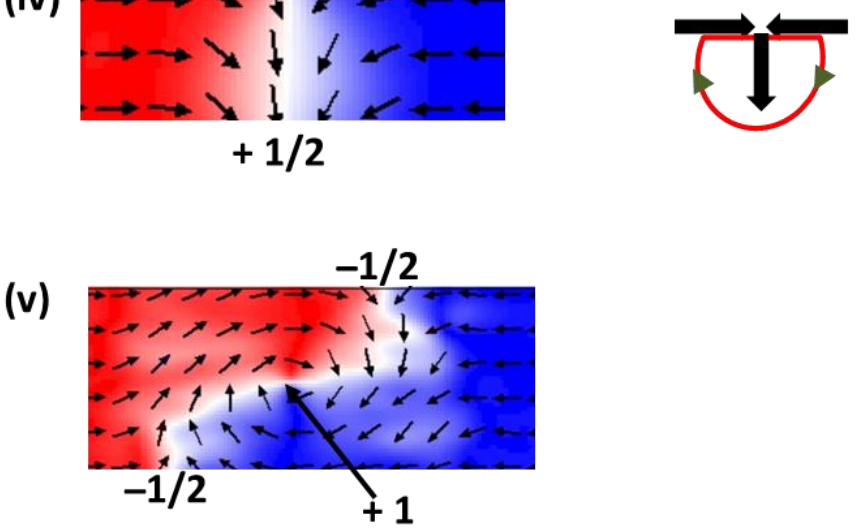

Figure 3-1 (a) The order parameter space around a topological defect in two dimensional plane represented by a circle (b) Topological defects in domain walls and their chirality (i) Tail-totail 'up' (TT-U), spins rotate clockwise; (ii) Tail-to-tail 'down' (TT-D), spins rotate anticlockwise; (iii) Head-to-head 'up' (HH-U), spins rotate anti-clockwise; (iv) Head-to-head 'down' (HH-D), spins rotate clockwise; (v) Vortex domain wall with clockwise chirality having bulk defect $=+1$ and two edge defects $=-1 / 2$. 
loop, the winding number at the base of the triangle is $+1 / 2$. This is equivalent to stating that the winding number or topological edge defect is $-1 / 2$, when two spins diverge and one converge at the point; the edge defect is $+1 / 2$ when all spins diverge or converge at the point. Similarly the topological defects are shown for other configurations of the transverse DW in Figure 3-1b (ii-iv). Figure 3-1b (ii) shows the defects for tail-to-tail down (TT-D) configuration where spins rotate in anti-clockwise direction. Figure 3-1b (iii) shows the head-to-head up (HH-U) configuration where the spins rotate in the anti-clockwise direction. Finally Figure 3$1 \mathrm{~b}$ (iv) shows the head-to-head down (HH-D) configuration where spins rotate in clockwise direction. For the case of wider nanowires where magnetostatic energy dominates, vortex DWs are stabilized [17-19]. The vortex DW has a defect in the bulk with winding number +1 (even though the spins rotate clockwise in this figure) and two defects at the edges with winding numbers $-1 / 2$, as shown in Figure 3-1 b(v). It is worth noting that the total winding number of topological defects in a DW add up to zero and the total topological charges or winding numbers are conserved during DW transformations [8].

Tchernyshyov et al. [8] also pointed out that the fractional winding numbers only exist at the edges and not inside the bulk, since that would cause the order parameter, $\theta$, to take multiple values. The multivaluedness would mean that the magnetization would be discontinuous across a line defect extending from the core of the half vortex to the film boundary, which would provide a potential confining the half vortex to the edge. From the electrostatic analogy, the defects $+1 / 2$ and $-1 / 2$ would attract each other, which would hold the composite DW together. The extent of the DW is set by the width of the strip, which is also reported in simulations [17]. For wider strips, the magnetostatic energy would break the symmetry between positive and negative winding numbers. The $+1 / 2$ winding number would have larger magnetostatic energy than the $-1 / 2$ defect [17]. The magnetostatic energy is minimized if the density of magnetic charges in the bulk vanishes i.e. $\nabla \cdot \widehat{m}=0$, and on the surface $\widehat{m}$ is parallel to the boundary. 
Since $+1 / 2$ defects are energetically unfavourable, in such cases it is possible that $+1 / 2$ defect would decay into a vortex $(n=+1)$ and an edge defect $(n=-1 / 2)$. In the exchange limit, such as for narrow width of nanowires, the $+1 / 2$ defect is a stable configuration. An antivortex is also a possible scenario which consists of bulk defect, $n=-1$ and edge defects $=+1 / 2$. However, this configuration is energetically unstable due to presence of two $+1 / 2$ defects. The annihilation of DWs is possible if edge defects can also annihilate following the principle of conservation of topological charge. To explain this situation consider a ring shaped nanowire magnetized such that the inner surface of the ring has two $-1 / 2$ charges. The DWs cannot annihilate since two $-1 / 2$ charges cannot cancel. This is exactly what happens in the case of narrow width rings. However, if the width of the ring is large there is a possibility of $+1 / 2$ charge to transform to a $-1 / 2$ charge by emitting a vortex with +1 charge. The emitted vortex propagates to the inner edge where it can recombine with the $-1 / 2$ defect to form $a+1 / 2$ defect. Now the defects can directly annihilate. In thin rings, the annihilation is less probable since the vortex is not energetically stable.

\subsection{Evolution of domain-wall at the bifurcation - a micromagnetic study}

The DW dynamics in a Y-branch structure was first investigated using object oriented micromagnetic framework (OOMMF) [20]. The branches were selected to have an angle of $70^{\circ}$ with respect to the horizontal $x$-axis. This value was selected based on simulations which showed that increasing the angle beyond $70^{\circ}$ pinned the DW at the bifurcation and further increasing the field did not depin it. On the other hand, if the angle is lower, the two branches themselves switch before the DW reaching the bifurcation. Hence, to improve the field resolution for DW chirality detection, $70^{\circ}$ was found to be optimum. The widths of the nanowires in the branch structure were chosen as $100 \mathrm{~nm}$ to stabilize a transverse DW [17-19]. The horizontal length of the nanowire where a DW is initialized is kept less than $200 \mathrm{~nm}$ to 
prevent Walker breakdown [21]. The simulation parameters for the permalloy were selected as following: exchange constant $A=1.3 \times 10^{-11} \mathrm{~J} / \mathrm{m}$, saturation magnetization $\mathrm{M}_{\mathrm{s}}=860 \times 10^{3}$ $\mathrm{A} / \mathrm{m}$ and anisotropy constant $\mathrm{K}=0 \mathrm{~J} / \mathrm{m}^{3}[18,22]$. The Gilbert damping coefficient $\alpha$ was selected as 0.01 . The simulation cell size was set as $5 \times 5 \times 5 \mathrm{~nm}^{3}$ along the three axes.
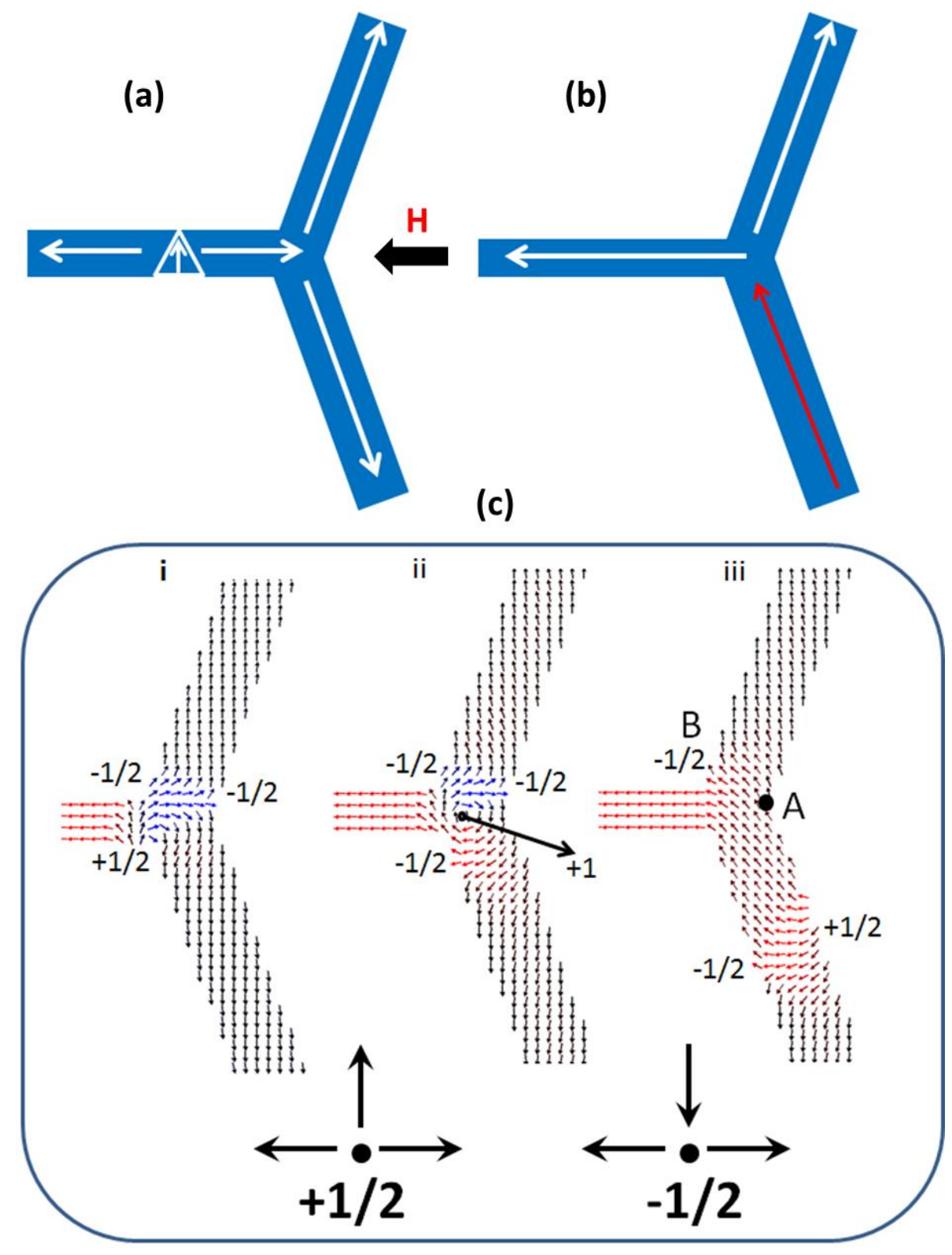

Figure 3-2 Schematics of the magnetization orientations in the network structure when a tailto-tail transverse DW with an "UP" chirality (TT-U) is injected: (a) before and (b) after the TT-U is propagated through the bifurcation. (c i-iii) Snap shots of the simulated magnetization configurations during the reversal process. Images are labelled with corresponding winding 
numbers of the edge defects. Point " $\mathrm{A}$ " indicates the position of the vertex at the bifurcation where the annihilation of vortex core occurs. Point " $B$ " represents the final position of the edge defect $n=-1 / 2$ at the bifurcation. Shown below are the schematic representations of elementary edge defects with winding numbers $+1 / 2$ and $-1 / 2$. Copyright (2014) The Japan Society of Applied Physics.

Figure 3-2 (a) shows the schematic of the magnetization configuration of the network or branch structure as a TT-U DW is initialized and driven in the nanowire. A field is applied to drive the DW, which then propagates selectively along the lower branch as shown in Figure 32 (b). The spin evolution at the bifurcation was obtained to understand the selective trajectory of the DW. The simulated magnetization configurations at the bifurcation under the application of gradually increasing field are shown in Figure 3-2 (c-(i-iii)). The figure also includes the winding numbers of the topological defects or charges of the representative spins. At the bifurcation, an edge defect of $-1 / 2$ is also observed, which is termed as the vertex. The DW upon reaching the bifurcation is pinned before it interacts with the vertex. A transverse DW has a clockwise orientation for 'up' chirality, hence when it approaches the bifurcation, the spins adopt a clockwise orientation. This pushes the vertex core towards the upper branch. Due to the transverse variation in DW width, the DW has a higher energy at the $+1 / 2$ defect than at the $-1 / 2$ defect. As the magnetic field strength increases, the bottom part of the DW depins and its interaction with the vertex leads to the formation of a vortex DW as shown in Figure 3-1 (cii). The vortex is stable state at the bifurcation due to dominant contribution of magnetostatic energy. The total winding number is conserved during the transformation, and the vortex is characterized by a +1 defect at the core and two $-1 / 2$ defects at the edges. The vortex core is formed after the transformation of the $+1 / 2$ defect from TT-U to +1 defect. Also, $a-1 / 2$ defect is nucleated along the same edge of the transformed $+1 / 2$ defect to conserve the total winding number. Gradual increase of magnetic field strength leads to the vortex DW to move towards point A, where the core eventually annihilates as shown in Figure 3-1(c-iii). As per topological charge conservation, the annihilation of the vortex core $(+1$ defect) leaves behind a $-1 / 2$ defect 
at the bifurcation at point B and a new transverse DW is nucleated in the lower branch as shown in Figure 3.1 (c-iii). The propagation of transverse DW along the lower branch switches the magnetization. In summary, the transformation process is completed by displacement of edge
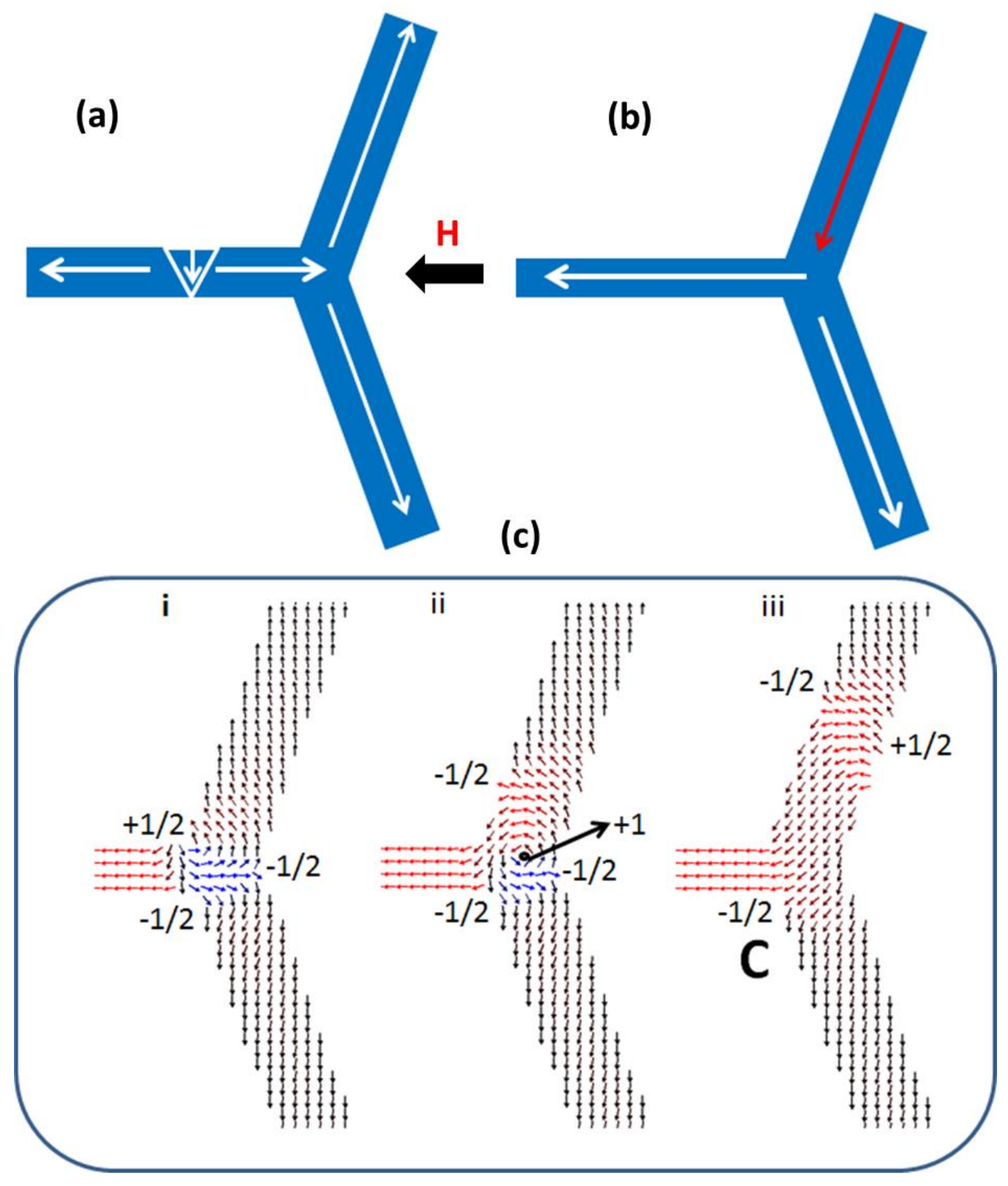

Figure 3-3 Schematics of the magnetization orientations in the network structure when a tailto-tail transverse DW with a "DOWN" chirality (TT-D) is injected: (a) before and (b) after the TT-D is propagated through the bifurcation. (c i-iii) Snap shots of the simulated magnetization configurations during the reversal process with winding numbers of the edge defects identified. Point " $C$ " represents the final position of the edge defect $n=-1 / 2$ at the bifurcation. Copyright (2014) The Japan Society of Applied Physics. 
defect from A to B.

The chirality of DW was now changed to tail-to-tail down (TT-D) and the above simulations were repeated. Figure 3-3 (a) shows the schematic of magnetization configuration when a TT-D DW is nucleated in the Y-branch structure. The application of in-plane magnetic field leads to similar dynamics as that presented for TT-U previously. However, on this occasion, the DW propagates along the upper branch as shown in the schematic of Figure 3-3 (b). In the case of TT-D DW, the upper edge has $+1 / 2$ defect and the bottom edge has $-1 / 2$ defect. The spins in the TT-D DW rotate in anti-clockwise direction. The vertex is displaced towards the lower branch as shown in Figure 3-3 (c-i). The depinning process of the transverse DW leads to the formation of an anti-clockwise vortex DW. This transformation takes place as a result of the annihilation of the $+1 / 2$ defect and generation of $a-1 / 2$ defect in the upper branch as shown in Figure 3-3 (c-ii). As the magnetic field increases, the vortex DW annihilates, leaving behind $-1 / 2$ defect at the position $\mathrm{C}$ and a transverse DW in the upper branch. Thus motion of DW in the upper branch switches its magnetization. The edge defect is displaced from position A to position C. From the above analysis we can infer that the DW always propagates in the branch where initial $+1 / 2$ defect is present. Thus the chirality of the DW dictates the switching behaviour and the trajectory of the DW.

\subsection{Direct observation of chirality dependent domain-wall trajectory}

Figure 3-4 (a) shows the scanning electron microscopy (SEM) image of the fabricated Ybranch structure. The structure is composed of thin film stack of Ta $(5 \mathrm{~nm}) / \mathrm{Ni}_{80} \mathrm{Fe}_{20}(10 \mathrm{~nm}) / \mathrm{Ta}$ $(5 \mathrm{~nm})$, deposited using magnetron sputtering at a base pressure of $2 \times 10^{-8}$ Torr. The film is patterned using electron beam lithography and Ar ion-milling techniques. In addition to the Ybranch nanowires the structure also has a diamond shaped pad for DW nucleation and injection along with a transverse nanowire for setting the initial chirality of the injected DW. It has been 
shown that the presence of a transverse nanowire also enhances the fidelity length of the DW to around $500 \mathrm{~nm}$ at an applied field strength of 50 Oe [21], since the transverse nanowire has a fixed magnetization owing to a strong shape anisotropy and it resets the chirality of the injected DW. We have confined the length of our nanowire to less than $400 \mathrm{~nm}$ to preserve the spin structure. The initial magnetization direction of the transverse nanowire selects the chirality of the injected DW. Thus the transverse nanowire acts as a chirality selector.

The experimental procedure for nucleating and driving a DW in the structure is described as follows. First, the transverse nanowire or the chirality selector is magnetized along $y$ direction using a large field of around 1-2 kOe. The field required to saturate the chirality selector is large since the width of the nanowire is less. The injected DW can either have up or down chirality depending on whether the chirality selector is magnetized along the $+y$ or $-y$ direction respectively. Next, the Y-branch is magnetized by applying 1 kOe field along the $+x$ direction. To inject a TT-DW, magnetic field is applied along the $-x$-direction. As the field is gradually increased, the DW is nucleated in the diamond pad and pushed inside the horizontal nanowire after passing through the chirality selector. Thus, the DW of a certain chirality is driven away from the pad and it propagates to one of the branches, thereby switching its magnetization. The procedure described above was visualized using magnetic force microscopy (MFM) imaging technique. Figure 3-4 (b-i) shows the MFM image of the structure when the chirality selector and the Y-branch nanowire structure are magnetized along the $+y$ and $+x$-directions respectively. As per our convention, the dark contrast indicates magnetic North Pole and the bright contrast indicates magnetic South Pole. Since, the spins inside a magnetic structure points from South to North, thus the arrows in Figure 3-4 (b-c) indicate the magnetization direction. When an in-plane magnetic field of strength 50 Oe is applied along the $-x$-direction, a TT-U DW is injected from the pad and reaches the bifurcation where it gets pinned due to low field strength. As the field strength is increased to $120 \mathrm{Oe}$, the DW overcome 

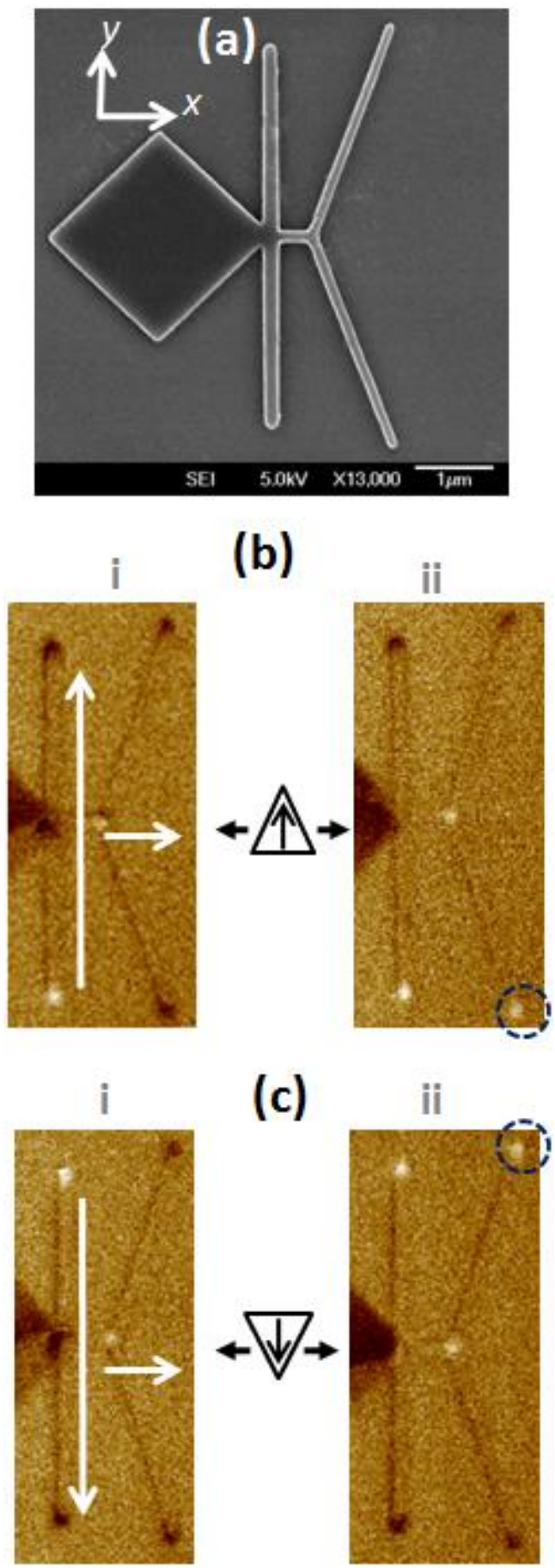

Figure 3-4 (a) Scanning electron microscopy image of the fabricated network structure. (b-i) Magnetic force microscopy (MFM) image of the initial magnetization configuration of the structure when the chirality selector is saturated with a $+y$ field, and (b-ii) the final magnetization configuration after a TT-U is injected and driven towards the bifurcation. (c-i) MFM image of the initial magnetization configuration of the structure when the chirality selector is saturated with a $-y$ field, and (c-ii) the final magnetization configuration after a TT$\mathrm{D}$ is injected and driven towards the bifurcation. Copyright (2014) The Japan Society of Applied Physics. 
the pinning potential and propagates into the lower branch as can be seen by the change in contrast of the lower branch from dark to bright. The contrast of the upper branch remains dark. Next, to inject and observe the trajectory of a TT-D DW, the magnetization of chirality selector was changed to point along $-y$-direction as shown in Figure 3-4 (c-i). Following the above procedure we observe from the MFM image shown in Figure 3-4 (c-ii) that the DW propagates along the upper branch. These findings indicate that the chirality of the transverse DW can govern the selective switching at either branch of the network. The MFM results correlate with the simulation results.

To obtain the magnetization configuration at the bifurcation during the transformation process, further detailed MFM scanning was performed with gradual increase in the field strength. Figure 3-5 (a) shows the MFM image of the device after initial magnetization but before DW injection. The triangular shape of the bright contrast indicates the presence of vertex at point A. The simulated magnetization configuration is shown below for comparison. After DW is injected and the field is gradually increased, it approaches the bifurcation where it is transformed into a vortex. This is shown in Figure 3-5 (b) as a circular contrast at the bifurcation. The low magnetic contrast can be attributed to the flux closure at the vortex [23]. With further increase in the magnetic field strength, the DW selectively propagates into one of the two branches, which is along the upper branch for this case since the injected DW is TTD. Figure 3-5 (c) shows the edge defect is now located at point C. This is a direct evidence of edge defect displacement and conservation of topological charge during DW transformation and propagation along the network structure. 


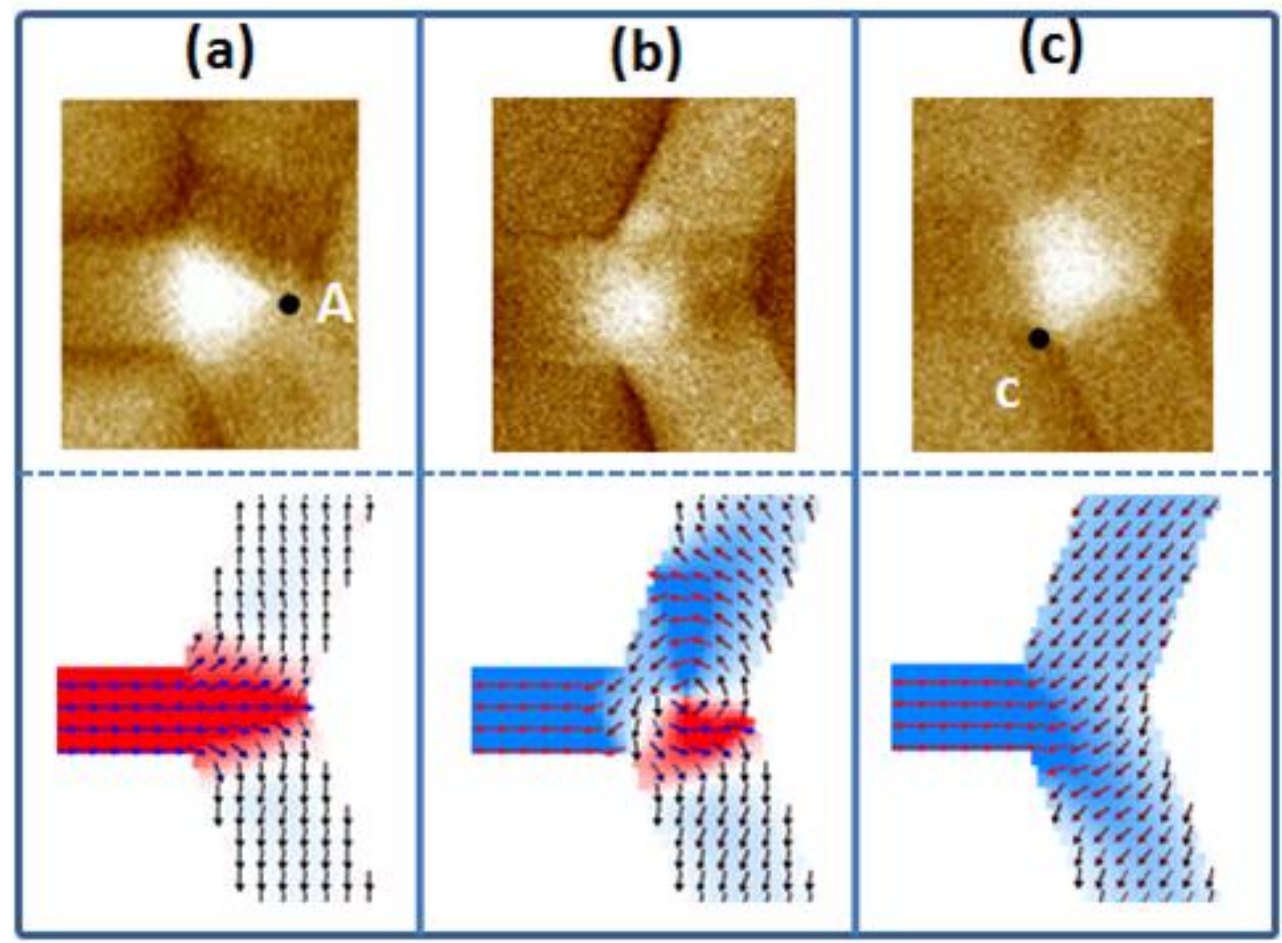

Figure 3-5 (a) MFM image of the network structure at the bifurcation prior to the DW injection. (b) MFM image after the DW reaches the bifurcation and (c) MFM image of the bifurcation after the DW motion through the network structure. Below are the snap shot images of the simulated magnetization configurations. Copyright (2014) The Japan Society of Applied Physics.

\subsection{Observation of Walker breakdown and stochastic trajectory of domain-}

\section{wall in network structures}

For logic devices based on DW trajectory in network structures, the output can be defined as the magnetization direction of the branches. In order to associate the magnetization along $x$ direction as the output, the branches need to be parallel to the $x$-axis. Hence, further study was devoted to a ' $U$ '-shaped branch structure instead of the ' $Y$ '-shaped structure. Simulations were performed to observe the DW trajectory in the 'U'-shaped branch structure. The length of the nanowire conduit carrying the DW was increased beyond the fidelity length to $1 \mu \mathrm{m}$ and the simulations were performed at two different field strengths. 

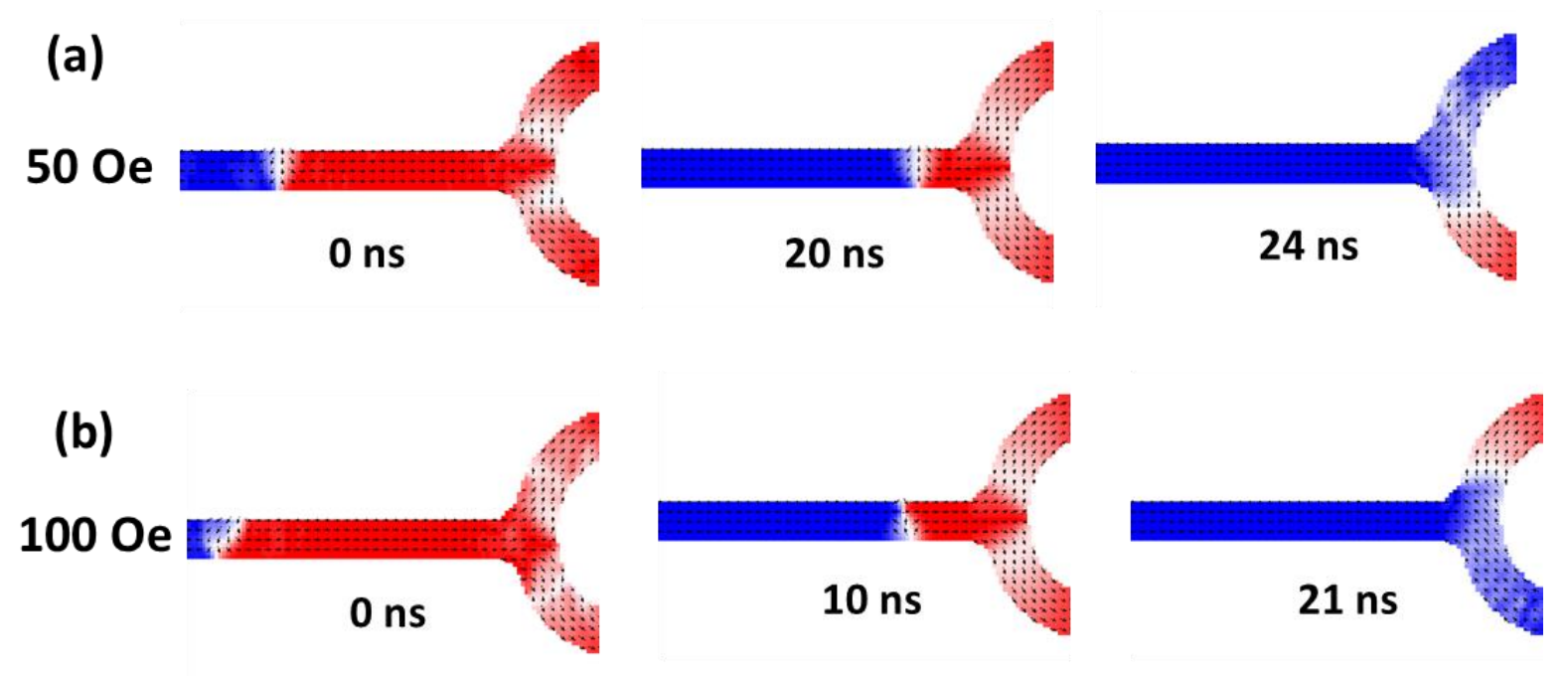

Figure 3-6 Simulated magnetization configuration when tail-to-tail 'down' (TT-D) DW is propagated in the branch structure at two different field strengths. (a) When field is 50 Oe, TTD propagates along the upper branch as expected. (b) When field strength is $100 \mathrm{Oe}$, Walker breakdown occurs as shown by anti-vortex configuration at $10 \mathrm{~ns}$, the DW propagates along the lower branch.

Figure 3-6 (a) shows the simulated magnetization configurations at different simulation times when the applied field is 50 Oe. When the DW approaches the bifurcation it pins there due to a potential barrier. To overcome this pinning strength the field was increased to $250 \mathrm{Oe}$ to enable further DW propagation. It is worth noting that the field required to overcome the pinning potential in simulations is much higher than that in experiments since simulations are performed at $0^{\circ} \mathrm{K}$ whereas in experiments, thermal effects reduce the pinning strength. This effect can be quantized by modifying the effective field in the LLG equation to include a thermal field term equivalent to $\boldsymbol{H}_{t h}=\boldsymbol{\eta} \sqrt{\frac{2 \alpha k_{B} T_{\mu M}}{\gamma \mu_{0} M_{S} \Delta x^{3} \Delta t}}$, where, $k_{B}$ is the Boltzmann constant, $\mathrm{T}_{\mu \mathrm{M}}$ represents the effective micromagnetic temperature, $\Delta x$ represents the cell size and $\Delta \mathrm{t}$ is the time step used to numerically integrate the Langevin equation and $\boldsymbol{\eta}$ is the vector representing stochasticity or fluctuation due to the temperature. Thermal fluctuations significantly reduce the minimum field, which promotes DW depinning as compared to the deterministic case at $\mathrm{T}=0{ }^{\circ} \mathrm{K}$. A TT-D DW is driven and propagates along the upper branch 
since it retains its chirality. Figure 3-6 (b) shows the simulated configurations when the applied field is increased to 100 Oe. At $10 \mathrm{~ns}$, it is observed that the TT-D DW breaks down to an antivortex configuration on account of Walker breakdown and obtains a TT-U chirality before entering the bifurcation (not showed here). Hence in the final configuration, after applying assist field of 250 Oe, the DW propagates along the lower branch instead of the upper branch. Thus we observe Walker breakdown leading to stochasticity in the DW trajectory.

Recent reports suggest vortex DW to be a more robust configuration than transverse DW [9]. Also, the vortex DW requires lesser field strength or depinning field to propagate through a bifurcation since the nanowire conduits are larger in widths and there is lesser shape anisotropy. We selected vortex DW as opposed to transverse DW in our quest to obtain deterministic DW trajectory and demonstrate a logic device. We performed a series of MFM measurements on an array of branch structures to get a statistical estimate of DW trajectory set with an initial chirality. The width of the nanowire was kept as $300 \mathrm{~nm}$ to stabilize a vortex DW. Figure 3-7 (a) shows MFM images depicting final magnetized configurations of 16 devices. We performed 30 such measurements and data is shown for 6 of those measurements in Figures 3-7 (a-f). The device is composed of "U"-shaped branch structure and a circular pad for DW nucleation. In the initial condition the device is magnetized along the $+y$ and $-x$ directions. When the field is gradually increased along the $+x$-direction a head-to-head $(\mathrm{HH})$ DW, with anti-clockwise chirality (ACW) is injected and driven in the nanowire conduit. The chirality of the injected vortex DW depends on the magnetization direction of the chirality selector as explained subsequently. The ACW DW is expected to move along the lower branch [9] and change the magnetization contrast from bright to dark. The initial magnetization contrast of both the branches is bright since HH-DW is initialized. However, in some of the cases the DW propagated along the upper branch (marked with ' $\mathrm{X}$ '). A statistical analysis was performed to get a measure of successes in the trials. Figure 3-8 shows the relative distribution 
of successes and failures in the experiments. We performed t-test to obtain a $95 \%$ confidence level. The range of successful trials was found to be $65 \%-75 \%$. Thus when a DW propagates along a branch structure it may change its chirality and lead to stochastic trajectory. We need to modify the structure in a manner that the trajectory becomes deterministic in order to perform successful logic operations. Before proposing structural modification we would look at how the chirality and the trajectory of the injected vortex DW depends on the magnetization of the chirality selector.

(a)

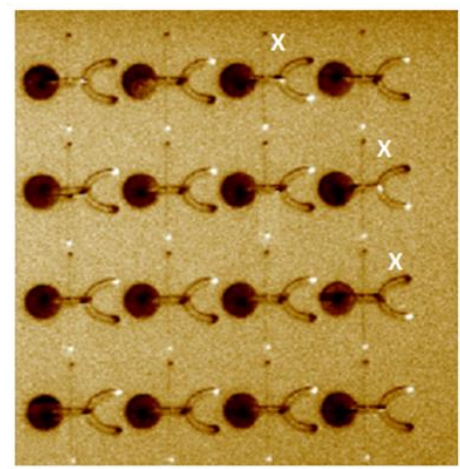

(c)

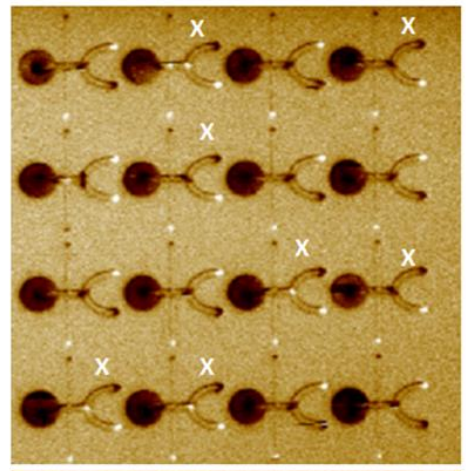

(e)

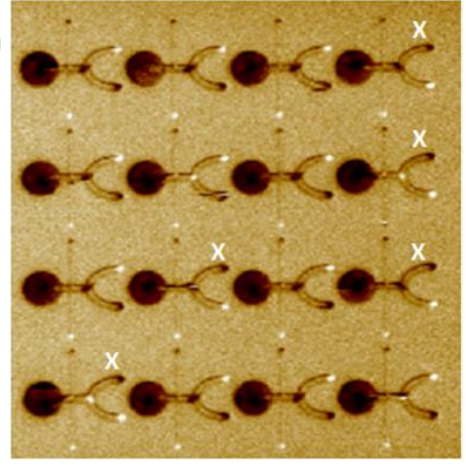

(b)

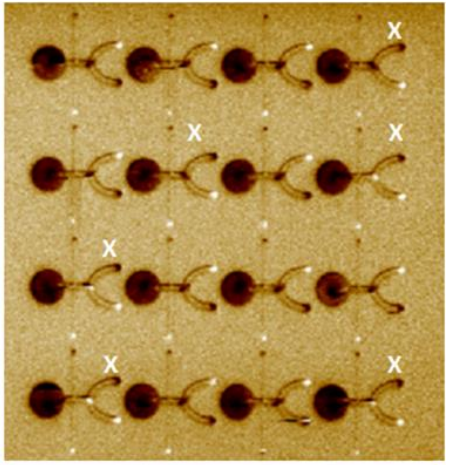

(d)

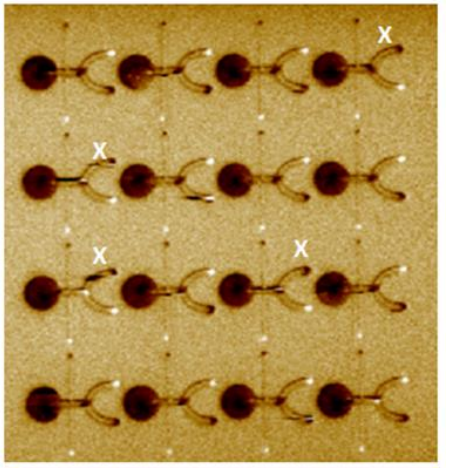

(f)

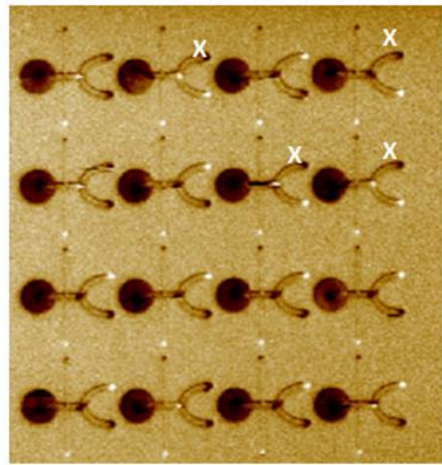

Figure 3-7 (a-f) MFM imaging to depict trajectory of anti-clockwise (ACW) vortex DW in a branch structure. The expected trajectory is along the lower branch where the contrast should be dark since initial contrast is bright for both branches. The ' $\mathrm{X}$ ' markings indicate propagation of DW along the upper branch indicating Walker breakdown leading to stochastic trajectory of 
the DW. The measurements were repeated 30 times. The six sets shown here are six of those measurements.

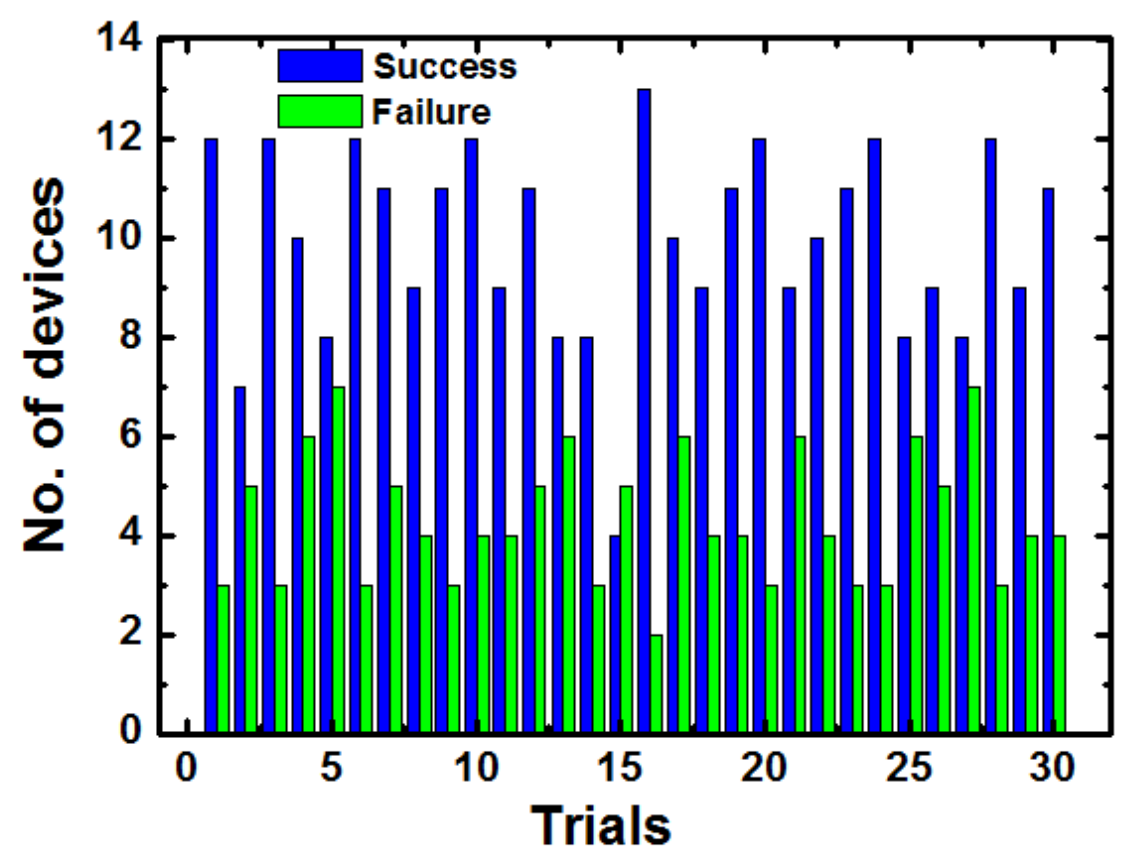

Figure 3-8 Relative distribution of successes and failures with success representing number of devices exhibiting chirality dependent selective trajectory of DWs.

\subsection{Chirality of vortex domain-wall in network structure}

The chirality of the injected vortex DW depends upon the magnetization direction of the chirality selector or the transverse nanowire which was demonstrated using simulations. Figure 3-9 (a) shows the initial magnetization configuration when the chirality selector is magnetized in the $+y$-direction. The horizontal nanowire is magnetized along the $-x$-direction. As field is applied along the $+x$-direction, HH DW is injected and takes an ACW chirality as shown in the simulated configuration of Figure 3-9 (b). Similarly Figure 3-9 (c) shows the initial magnetization configuration when the chirality selector is magnetized along the $-y$-direction. On this occasion the injected DW takes up a clockwise (CW) chirality as shown in Figure 3-9 (d). 
(a)
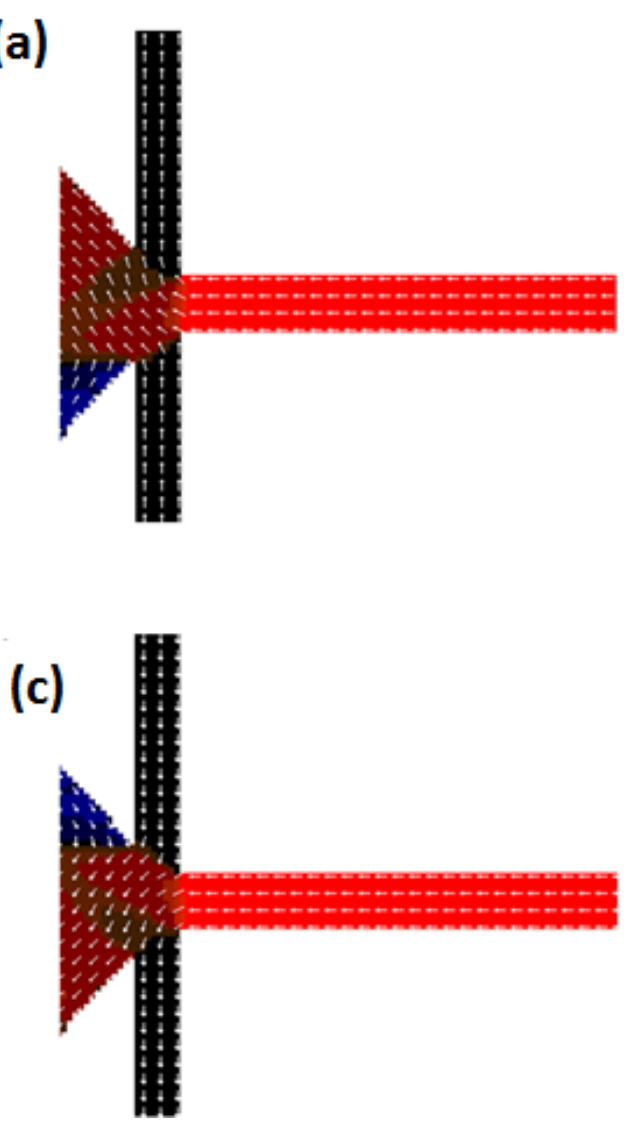

(b)

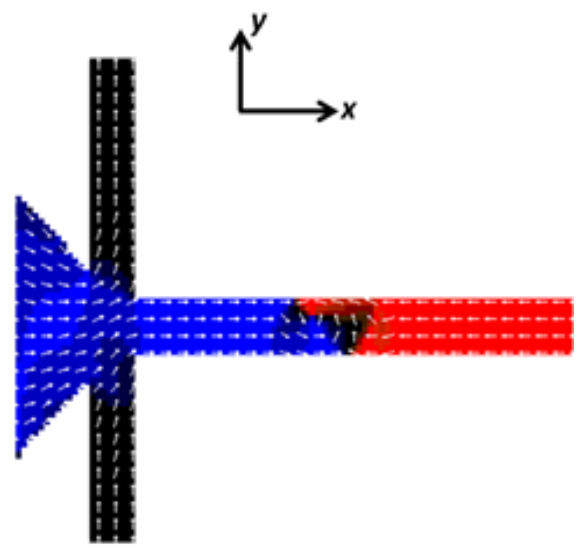

(d)

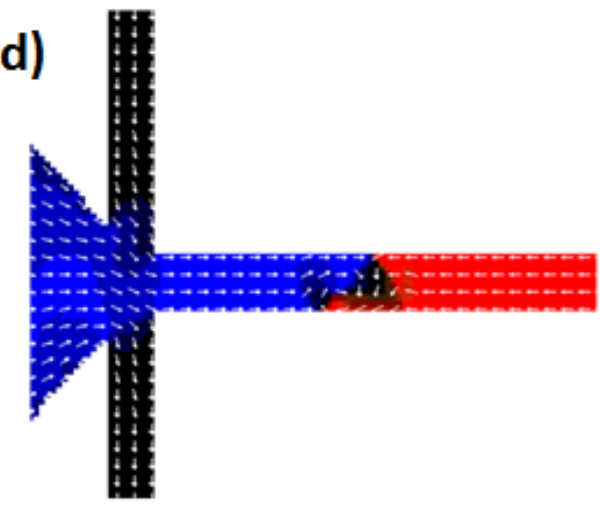

Figure 3-9 Chirality of injected vortex DW as a function of initial magnetization direction of the transverse nanowire or the chirality selector. (a) Chirality selector magnetized along $+y$ direction (b) Injected DW is clockwise. (c) Chirality selector magnetized along $-y$-direction (d) Injected DW is anti-clockwise.

\subsection{Geometrical asymmetry to control trajectory of domain-wall in network}

\section{structure}

To control the trajectory of DW in network structure, we propose a modification to the device design by adding a geometrical asymmetry. This asymmetry provides a potential barrier to the propagation of the DW and constrains it to move along a particular branch only. The advantage of this approach is that the propagation can be extended beyond the fidelity length and the motion is independent of the DW chirality and hence immune to Walker breakdown. 


\subsubsection{Constrained motion of domain-wall along the upper branch}

Figure 3-10 (a) shows the schematic of the structure when the output branch is displaced along the $-y$-direction by an amount equal to $\mathrm{D}_{\text {offset. }}$ This geometrically modified structure is termed as 'pull-down' (PD). Figure 3-10b (i-iv) represents the simulated magnetization configurations
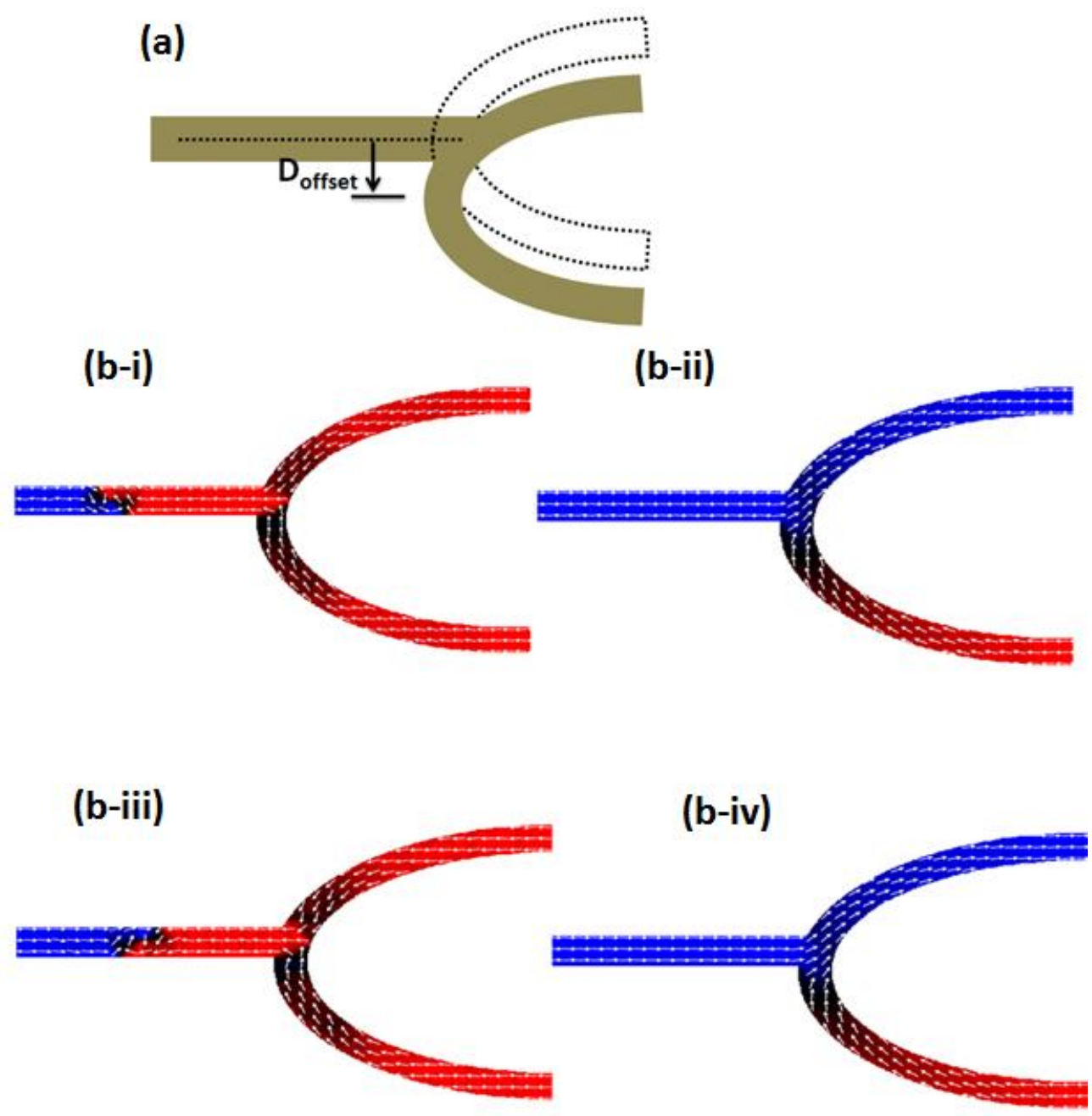

Figure 3-10 Trajectory of a domain wall in asymmetric branch structures. (a) Schematic of 'pull-down' (PD) structure, where output branch is displaced in the $-y$ direction and the offset is labelled as $D_{\text {offset. }}$ (b-i) Simulated initial magnetization configuration of the PD structure when ACW is injected in the nanowire. (b-ii) Simulated final magnetization configuration of the PD structure depicting DW moving to the upper branch. (b-iii) Simulated initial magnetization configuration of the PD structure when CW is injected in the nanowire. (b-iv) Simulated final magnetization configuration of the PD structure depicting DW moving to the upper branch. 
of the PD device for $\mathrm{D}_{\text {offset }}=200 \mathrm{~nm}$, when ACW and CW vortex DWs are propagated in the structure. Figure 3-10b (i) shows the initial magnetization of the device before driving an ACW vortex DW. The ACW vortex DW propagates along the upper branch switching the magnetization direction of the upper branch from $-x$ to $+x$-direction as shown in Figure 3-10b (ii). Figure 3-10b (iii) shows the initial magnetization configuration of the PD structure before driving a CW vortex DW. The CW vortex DW also propagates along the upper branch as shown in Figure 3-10b (iv). Thus, the DW propagates along the upper branch in the PD structure irrespective of its chirality.

Experiments were carried out using MFM imaging technique to support the simulation results. Figure 3-11 (a) shows the SEM image of the fabricated device. The device fabrication process and thin film employed is similar to the one described in section 3.3. The width of the nanowire and the output branch structure were kept as $300 \mathrm{~nm}$, however, the width of the transverse nanowire was maintained at $100 \mathrm{~nm}$ to increase the shape anisotropy. The distance between the transverse nanowire, also known as the chirality selector, and the output branch was kept as $1 \mu \mathrm{m}$. The $\mathrm{D}_{\text {offset }}$ was kept as $200 \mathrm{~nm}$. Figure 3-11b (i) shows initial MFM image of the device when the chirality selector and the branch structure are magnetized along the $+y$ and $-x$-directions, respectively. On gradually increasing the magnetic field in the $+x$-direction, HH DW with ACW chirality was injected and driven to the upper branch at a field strength of 65 Oe. Figure 3-11b (ii) shows the final magnetization configuration when the magnetic contrast of the upper branch switches from bright to dark. Figure 3-11b (iii) shows the final MFM image of an array of such PD structures. Out of 16 structures, 15 showed a successful trial where the DW propagated along the upper branch. However, repeated measurements on the failed device showed that the device does not fail every time and hence fabrication related defect can be ruled out. The failure could be due to thermal instabilities. Figure 3-11c (i) shows the initial MFM image when the chirality selector and the branch structure are magnetized 


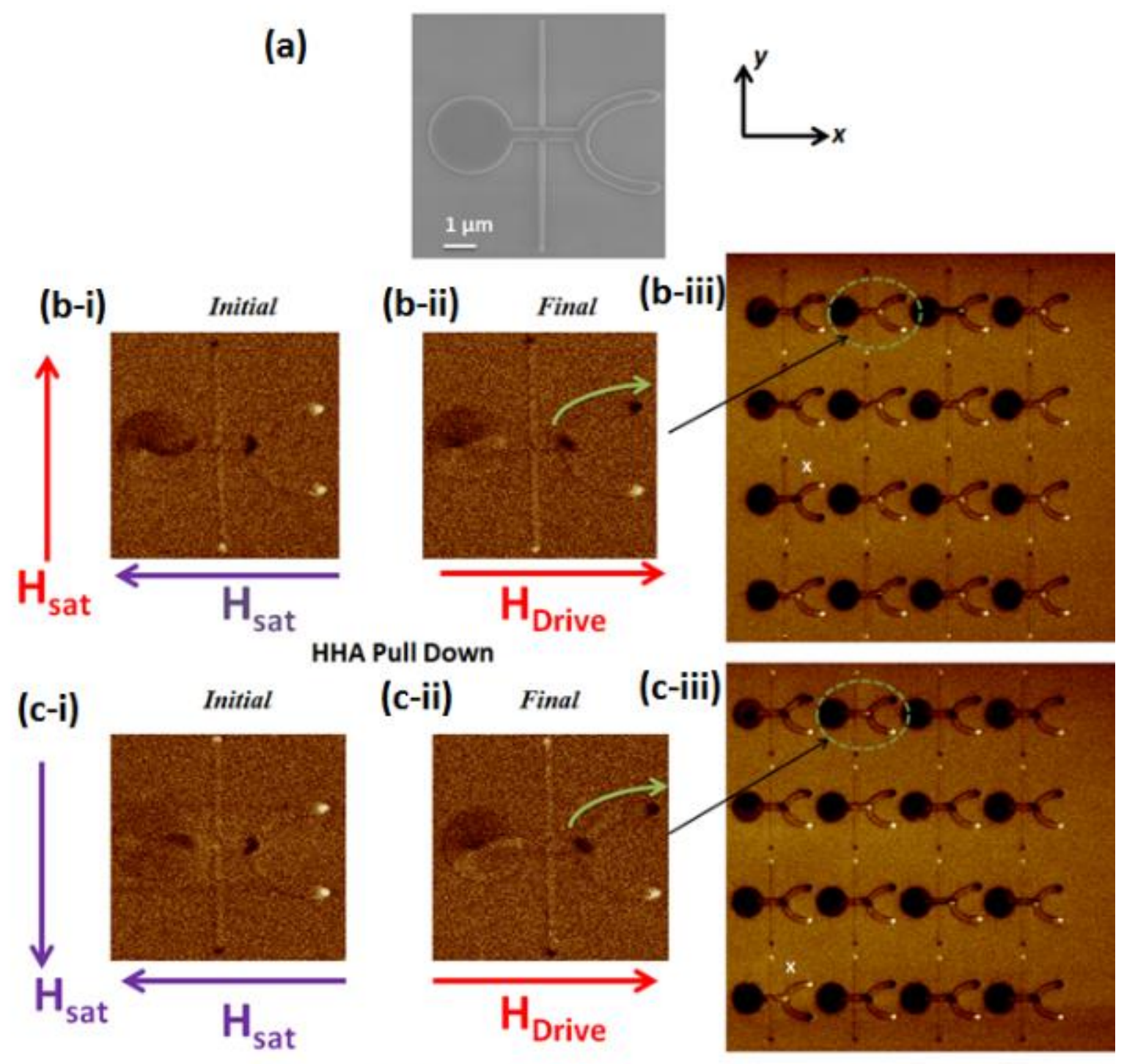

HHC Pull Down

Figure 3-11 Direct observation of vortex domain wall trajectory in the PD structure. (a) SEM image shows the PD structure, the output branch is displaced in the $-y$ direction by $200 \mathrm{~nm}$. (b-i) MFM image of initial magnetization configuration when transverse nanowire is saturated in the $+y$ direction and the output branch is saturated in the $-x$ direction. (b-ii) MFM image of final magnetization configuration when ACW is driven to the upper branch. (b-iii) MFM image of an array of PD structures. (c-i) MFM image of initial magnetization configuration when transverse nanowire is saturated in the $-y$ direction and the output branch is saturated in the $-x$ direction. (c-ii) MFM image of final magnetization configuration when $\mathrm{CW}$ is driven to the upper branch. (c-iii) MFM image of an array of PD structure. 
along the $-y$ and $-x$-directions respectively. On increasing the field in the $+x$-direction, a $\mathrm{HH}$ DW with CW chirality is injected and driven along the upper branch. The final MFM image is shown in Figure 3-11c (ii), where the magnetic contrast of the upper branch changes from bright to dark. Figure 3-11c (iii) shows the final MFM image of an array of such PD structures, here also similar to the previous case all except one device exhibit successful trial. The above measurements were repeated 20 times on a single PD device as well for both ACW and CW initial chirality configurations. It was observed that the DW always propagated along the upper branch. Although setting an initial chirality may be irrelevant since the final chirality at the bifurcation may be different from the initial one, this allows us to compare results for two different initial conditions. Thus by displacing output branch along the $-y$-direction we can constrain the DW motion along the upper branch.

\subsubsection{Constrained motion of the domain-wall along the lower branch}

Figure 3-12 (a) shows the schematic of the structure when the output branch is displaced along the $+y$-direction. This structure is termed as 'pull-up' (PU) structure. This is analogous to the PD structure with the DW now propagating along the lower branch due to the asymmetry and potential barrier along the upper branch. Simulations and experiments that were performed for the PD structure shown in the previous section were also repeated for the PU structure. Figure 3-12b (i-iv) shows the simulation results for ACW and CW vortex domain walls propagating along the lower branch. Figure 3-13 (a) shows the SEM image of the fabricated PU structure. Figure 3-13b (i-iv) show the MFM results of the PU structure when ACW and CW vortex DW propagate along the lower branch. Thus by displacing the output branch along the $+y$-direction, the DW can be constrained to propagate along the lower branch. 

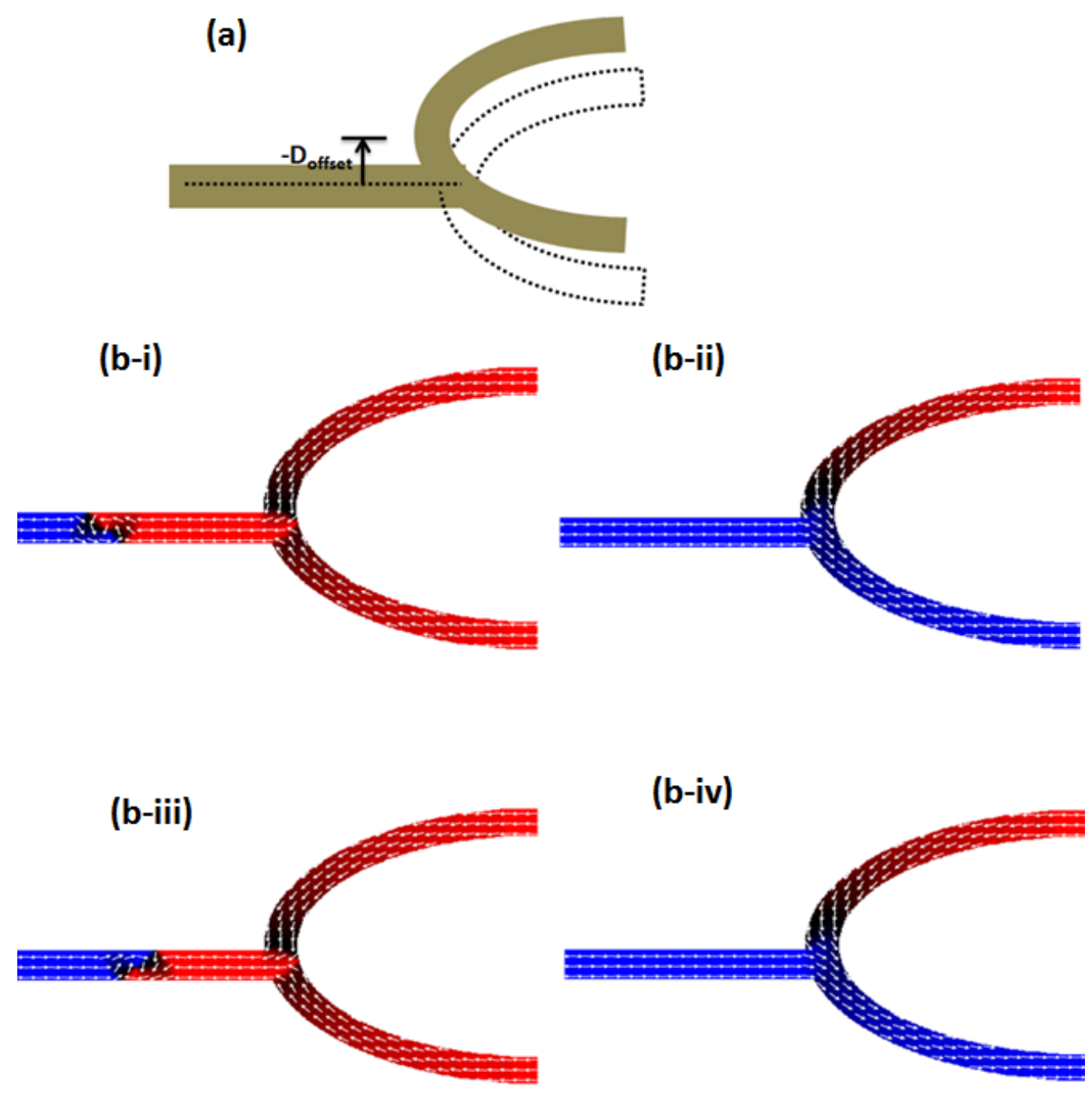

Figure 3-12 (a) Schematic of 'pull-up' (PU) structure, where output branch is displaced in the $+y$ direction and the offset is labelled as $D_{\text {offset. }}$ (b-i) Simulated initial magnetization configuration of the PU structure when ACW is injected in the nanowire. (b-ii) Simulated final magnetization configuration of the PU structure depicting DW moving to the lower branch. (biii) Simulated initial magnetization configuration of the PU structure when $\mathrm{CW}$ is injected in the nanowire. (b-iv) Simulated final magnetization configuration of the PU structure depicting DW moving to the lower branch. 
(a)
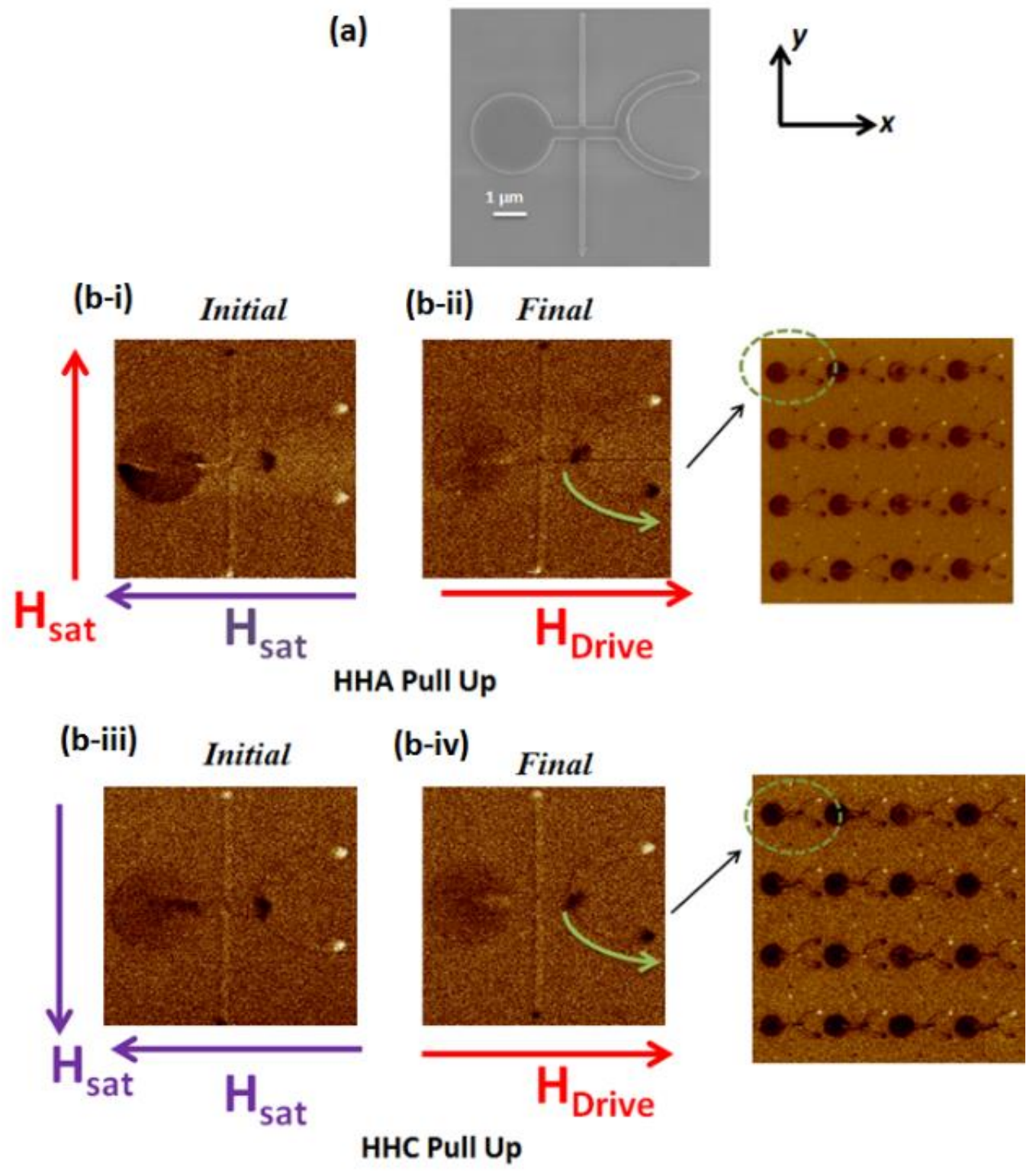

Figure 3-13 (a) SEM image shows the PU structure, the output branch is displaced in the $+y$ direction by $200 \mathrm{~nm}$. (b-i) MFM image of initial magnetization configuration when transverse nanowire is saturated in the $+y$ direction and the output branch is saturated in the $-x$ direction. (b-ii) MFM image of final magnetization configuration when ACW is driven to the lower branch. (b-iii) MFM image of initial magnetization configuration when transverse nanowire is saturated in the $-y$ direction and the output branch is saturated in the $-x$ direction. (b-iv) MFM image of final magnetization configuration when $\mathrm{CW}$ is driven to the lower branch. 


\subsubsection{Evolution of vortex domain-wall at the bifurcation of asymmetric network structure}

The evolution of vortex DW at the bifurcation and its subsequent trajectory were analysed with the aid of topological charges. As presented in section 3.1, a vortex DW is composed of a bulk defect of a winding number +1 at the core and two edge defects of winding number $-1 / 2$. Figure 3-14 (a) shows the simulated magnetization configuration at the bifurcation for symmetric and PD structure when an ACW vortex DW is propagated in the structures. The combination of PD structure with ACW vortex DW is selected to demonstrate DW trajectory against the chirality determined path. Since in a symmetric structure the ACW DW would propagate along the lower branch but in PD structure, the trajectory of DW would be along the upper branch. The other combinations are generalization of this particular combination. An edge defect of $-1 / 2$ exists at the bifurcation in both the structures denoted by $V_{S}$ and $V_{A}$ for symmetric and asymmetric structures respectively in Figure 3-14 (a). The position of this edge defect is at the centre $\left(V_{S}\right)$ with the respect to the horizontal nanowire for the case of symmetric structure. In the asymmetric structure, the position of the edge defect is intrinsically pushed towards the lower branch $\left(\mathrm{V}_{\mathrm{A}}\right)$.

Consider the DW evolution at the bifurcation for the symmetric structure as magnetic field is gradually increased. The interaction between the vortex DW at the bifurcation and the intrinsic magnetic texture present at the vertex, displaces the $-1 / 2$ defect towards the lower branch from $\mathrm{V}_{\mathrm{S}}$ to $\mathrm{V}_{\mathrm{S}^{\prime}}$ when the field strength is 58 Oe. When the field strength reaches 124 Oe, the vortex core enters the upper branch and rearrangement of topological charges take place. The vortex DW carries the $-1 / 2$ defect from $V_{s^{\prime}}$ and leaves behind $-1 / 2$ defect at point A. When the magnetic field is increased to $172 \mathrm{Oe}$, the vortex DW annihilates leaving behind $1 / 2$ defect at point $\mathrm{C}$. This process leads to the depinning of the $-1 / 2$ defect at point $\mathrm{A}$ and subsequent propagation of a new DW in the lower branch. The DW nucleation in the lower 


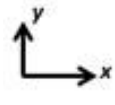

(a)
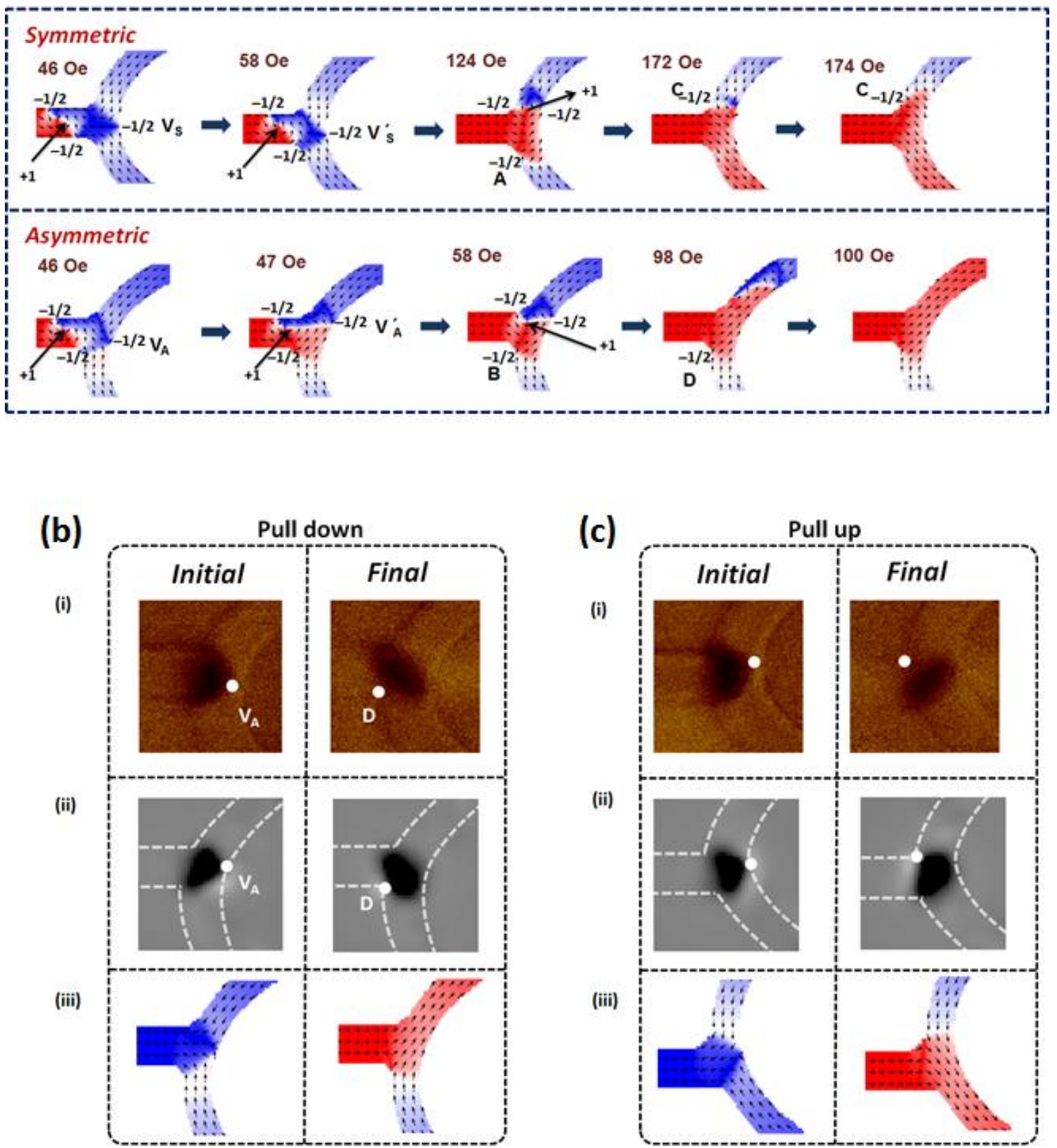

Figure 3-14 (a) Simulated magnetization configurations showing comparison of DW evolution at the bifurcation between symmetric and asymmetric structures (PD). The corresponding winding numbers are labelled and snapshots are taken at different field strengths. (b) Initial and final configurations at the bifurcation for PD structure (i) MFM image showing the vertex charge; (ii) Simulated MFM configurations at the bifurcation depicting the position of the vertex; (iii) Simulated spin configurations at the bifurcation. (c) Initial and final configurations at the bifurcation for PU structure (i) MFM image showing the vertex charge; (ii) Simulated MFM configurations at the bifurcation depicting the position of the vertex; (iii) Simulated spin configurations at the bifurcation. 
branch requires higher field. The net result of the DW motion through the branch structure is the displacement of $-1 / 2$ defect from $\mathrm{V}_{\mathrm{S}}$ to point $\mathrm{C}$.

Next consider the DW evolution at the bifurcation for the asymmetric PD structure as magnetic field is gradually increased. Geometrical asymmetry reduces the energy barrier towards the upper branch since it is less steep. The interaction of DW at the bifurcation with the magnetic texture at the vertex displaces the $-1 / 2$ defect towards the upper branch $\left(\mathrm{V}_{\mathrm{A}^{\prime}}\right)$. When the field strength is increased to 58 Oe, the vortex core enters the upper branch and rearrangement of topological charges take place. The vortex DW carries the $-1 / 2$ defect from point $\mathrm{V}_{\mathrm{A}^{\prime}}$ and leaves behind $-1 / 2$ defect at point $\mathrm{B}$. As the applied field reaches 98 Oe the vortex DW gets depinned and propagates along the upper branch, leaving behind $-1 / 2$ defect at point D. The net result of the DW motion through the branch structure is the displacement of $-1 / 2$ defect from $V_{A}$ to $D$.

Comparison between asymmetric and symmetric structures reveals that initially the vortex DW propagates along the upper branch for both the structures as shown in the third image comparison of Figure 3-14 (a). However, the depinning field of the DW in the symmetric structure is much larger than the asymmetric structure. The difference arises on account of the position of the $-1 / 2$ defect at the bifurcation after the DW approaches it. In symmetric structure$1 / 2$ defect is displaced towards the lower branch, whereas asymmetry displaces the $-1 / 2$ defect along the upper branch in the asymmetric structure. The vortex DW carries the $-1 / 2$ defect from the upper branch for the asymmetric structure as opposed to symmetric structure where the DW carries the defect from the lower branch, hence lesser field is required for the asymmetric structure. The difference is observed in the fourth image of Figure 3-14 (a), where in the case of symmetric structure, the vortex DW annihilates and a new DW is nucleated which propagates along the lower branch. For the case of asymmetric structure, the vortex DW itself 
depins and propagates along the upper branch. The asymmetry changes the potential landscape and plays an important role in guiding the DW to the respective branch. The total topological charge before and after the transformation is conserved and remains $-1 / 2$. The DW propagates in the branch opposite to the $-1 / 2$ defect left by the DW transformation. Similar dynamics approach explains the DW trajectory in the case of PU structure.

MFM imaging was carried out on the asymmetric structures to observe the spin configuration at the bifurcation. Figure 3-14b (i-iii) show the MFM images and the corresponding simulated magnetization configurations at the bifurcation for initial and final magnetization states in the PD structure. The MFM contrast in Figure 3-14b (ii) was simulated using MuMax tool $[24,25]$. Point A denotes the position of the intrinsic $-1 / 2$ defect pushed towards the lower branch. Point $\mathrm{D}$ denotes the position of the $-1 / 2$ edge defect left behind at the lower corner of the bifurcation as the DW propagates along the upper branch. The MFM images for the PU structure are shown in Figure 3-14c (i-iii). In comparison to the PD case, the intrinsic defect is pushed towards the upper branch. Also in the final magnetized state, the edge defect is positioned at the upper corner of the bifurcation as the DW propagates along the lower branch.

\subsubsection{Potential energy barrier at the bifurcation as a function of the geometrical asymmetry}

The influence of $\mathrm{D}_{\text {offset }}$ on the potential energy required to overcome the barrier due to asymmetry was quantified by performing micromagnetic simulations for different values of $\mathrm{D}_{\text {offset. }}$ The Zeeman energy which is a measure of the pinning strength against the propagation of DW into one of the branches was determined. Figure 3-15 shows the plot of Zeeman energy versus $D_{\text {offset }}$ when an ACW vortex DW is propagated in the PD structure. When $D_{\text {offset }}=0$, the 


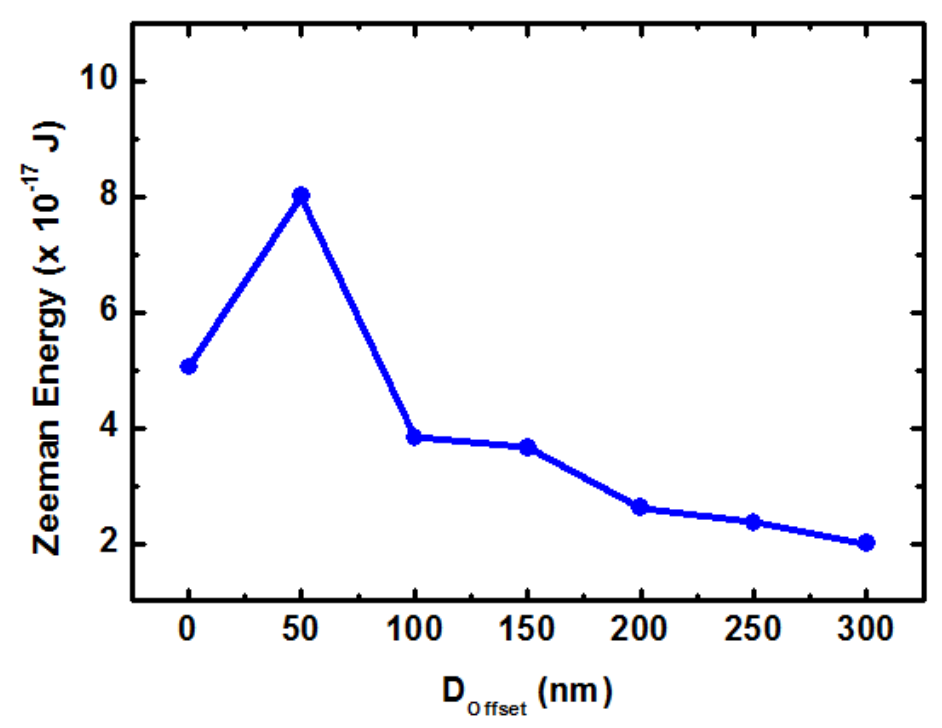

Figure 3-15 Plot of Zeeman energy required to move the DW in one of the two branches as a function of $\mathrm{D}_{\text {offset }}$. There is a competition between two potential energy barriers: one imposed due to selective trajectory and the other imposed due to geometrical asymmetry. Above 100 $\mathrm{nm}$ the potential barrier due to geometrical asymmetry in the PD structure is lower for the DW to move in the upper branch.

structure is symmetric. The DW propagates to the bifurcation as magnetic field is gradually increased and gets pinned at the bifurcation. The DW encounters two potential barriers along the upper and lower branch respectively. When the field increases, the DW propagates along the lower branch according to the chirality dependent selective trajectory [9]. As $\mathrm{D}_{\text {offset }}$ is increased along the downward direction, the potential barriers also change along the two directions. The DW experiences lesser potential barrier along the upper branch but the potential barrier increases along the lower branch due to geometrical asymmetry. However at low $\mathrm{D}_{\text {offset, }}$ the DW still choses to propagate along the lower branch as long as it can overcome the barrier due to asymmetry. From the simulations, it was observed that for $\mathrm{D}_{\text {offset }}<100 \mathrm{~nm}$, the DW choses to propagate along the lower branch i.e. it follows the same path as if the structure is symmetric. However, for $\mathrm{D}_{\text {offset }}>100 \mathrm{~nm}$, the DW cannot overcome the barrier experienced along 
the lower branch and it sees a weaker potential along the upper branch and hence propagates along the upper branch. The plot in Figure 3-14 also shows that the Zeeman energy increases when $\mathrm{D}_{\text {offset }}=50 \mathrm{~nm}$ after which it reduces. A possible explanation for this behaviour could be that below $100 \mathrm{~nm}$ the $\mathrm{D}_{\text {offset }}$ acts as a barrier and increases the pinning against the DW propagation along the lower branch which enhances the Zeeman energy. When $\mathrm{D}_{\text {offset }}$ is increased beyond $100 \mathrm{~nm}$, the barrier is higher towards the lower branch and less towards the upper branch. This reduces the energy for DW propagation along the upper branch. Thus there is a critical $\mathrm{D}_{\text {offset }}$ beyond which the asymmetry starts to dominate and influence the trajectory of the DW.

\subsubsection{Effect of initial vortex core polarity on the domain-wall trajectory}

As mentioned previously the vortex DW is characterized by bulk topological charge and two edge defect charges. The bulk topological charge also defines the vortex core polarity which can be either +1 or -1 . We also examined the effect of initial vortex core polarity on the DW trajectory in asymmetric structure using simulations. Figure 3-16 shows the comparison between the DW dynamics when ACW vortex DW propagates with an initial core polarity as positive and negative respectively in a PD structure. The evolution of DW at the bifurcation is similar to the one explained in section 3.6.3. The positive core maintained its polarity as the DW propagates along the upper branch. However, the negative core switches its polarity to positive while the vortex core enters the bifurcation. The vortex core with polarity $=-1$ is unstable as explained in section 3.1 Subsequently the DW also propagates along the upper branch. 


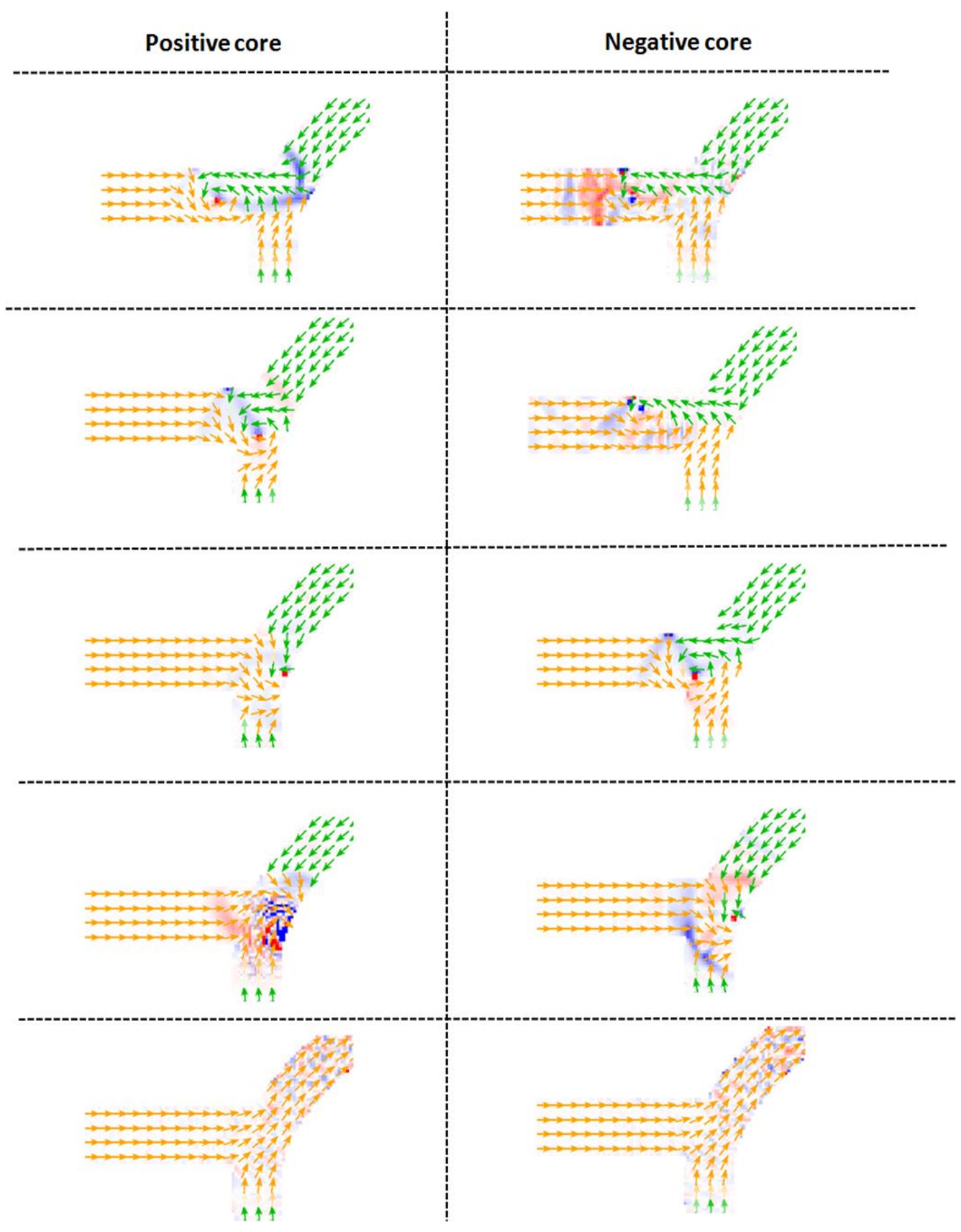

Figure 3-16 Comparison of DW trajectory in the PD structure between initial polarity of the core as positive and negative respectively. The trajectory is independent of the core polarity. The negative polarity of the vortex DW being unstable switches to positive polarity before propagating into the upper branch. 


\subsection{Summary}

Field induced DW dynamics were studied in network structures with in-plane magnetization anisotropy. The evolution of the DWs were examined at the bifurcation of network structures with the aid of topological defects or charges. The trajectory of transverse DW was found to be chirality dependent in a Y-branch structure. The transverse DW transforms to a vortex configuration as the DW enters the bifurcation. The annihilation of the vortex DW at high field subsequently nucleates a transverse DW in one of the two branches. The motion of DW through the network displaces the edge defect at the bifurcation. At larger applied field strengths and DW propagation over distances longer than the fidelity length, the DW chirality does not remain fixed and was found to vary on account of Walker breakdown. This led to stochasticity in the trajectory of DW across the network structure. Vortex DWs which are considered more robust than transverse DW and depin at lower field strengths were adopted as the preferred DW configuration. However, the vortex DWs were also found to undergo Walker breakdown. To achieve deterministic trajectory of the DWs in the network structure for logic applications a geometrical asymmetry was proposed to provide a potential barrier to the DW motion along one of the branches. Two geometrical modifications were proposed in a U-shaped network structure. In one of the geometries the output branch was displaced along the $-y$-direction to constrain DW motion along the upper branch; in the second modification the output branch was displaced along the $+y$-direction to constrain the motion of DW along the lower branch. The evolution of DW was studied at the bifurcation with the aid of topological defects. The DW trajectory became deterministic independent of vortex core polarity or chirality and Walker breakdown. These principles are applied to propose programmable logic device discussed in the next chapter. 


\section{Bibliography}

[1] D. A. Allwood, G. Xiong, C. C. Faulkner, D. Atkinson, D. Petit, and R. P. Cowburn, "Magnetic domain-wall logic," Science, vol. 309, pp. 1688-1692, Sep 92005.

[2] C. Murapaka, P. Sethi, S. Goolaup, and W. S. Lew, "Reconfigurable logic via gate controlled domain wall trajectory in magnetic network structure," Sci Rep, vol. 6, p. 20130, Feb 032016.

[3] R. F. Wang, C. Nisoli, R. S. Freitas, J. Li, W. McConville, B. J. Cooley, et al., "Artificial 'spin ice' in a geometrically frustrated lattice of nanoscale ferromagnetic islands," Nature, vol. 439, pp. 303-306, Jan 192006.

[4] S. Ladak, D. E. Read, G. K. Perkins, L. F. Cohen, and W. R. Branford, "Direct observation of magnetic monopole defects in an artificial spin-ice system," Nature Physics, vol. 6, pp. 359363, May 2010.

[5] P. Mellado, O. Petrova, Y. C. Shen, and O. Tchernyshyov, "Dynamics of Magnetic Charges in Artificial Spin Ice," Physical Review Letters, vol. 105, Oct 272010.

[6] C. Phatak, A. K. Petford-Long, O. Heinonen, M. Tanase, and M. De Graef, "Nanoscale structure of the magnetic induction at monopole defects in artificial spin-ice lattices," Physical Review B, vol. 83, May 182011.

[7] S. Zhang, I. Gilbert, C. Nisoli, G. W. Chern, M. J. Erickson, L. O'Brien, et al., "Crystallites of magnetic charges in artificial spin ice," Nature, vol. 500, pp. 553-557, Aug 292013.

[8] O. Tchernyshyov and G. W. Chern, "Fractional vortices and composite domain walls in flat nanomagnets," Physical Review Letters, vol. 95, Nov 42005.

[9] A. Pushp, T. Phung, C. Rettner, B. P. Hughes, S. H. Yang, L. Thomas, et al., "Domain wall trajectory determined by its fractional topological edge defects," Nature Physics, vol. 9, pp. 505-511, Aug 2013.

[10] K. Zeissler, S. K. Walton, S. Ladak, D. E. Read, T. Tyliszczak, L. F. Cohen, et al., "The nonrandom walk of chiral magnetic charge carriers in artificial spin ice," Scientific Reports, vol. 3, Feb 132013.

[11] N. L. Schryer and L. R. Walker, "Motion of 180 Degrees Domain-Walls in Uniform Dc Magnetic-Fields," Journal of Applied Physics, vol. 45, pp. 5406-5421, 1974.

[12] D. M. Burn, M. Chadha, S. K. Walton, and W. R. Branford, "Dynamic interaction between domain walls and nanowire vertices," Physical Review B, vol. 90, Oct 132014.

[13] I. Syozi, "Statistics of Kagome Lattice," Progress of Theoretical Physics, vol. 6, pp. 306-308, 1951.

[14] Y. C. Shen, O. Petrova, P. Mellado, S. Daunheimer, J. Cumings, and O. Tchernyshyov, "Dynamics of artificial spin ice: a continuous honeycomb network," New Journal of Physics, vol. 14, Mar 302012.

[15] P. M. Chaikin and T. C. Lubensky, Principle of Condensed Matter Physics: Cambridge University Press, 2000.

[16] N. D. Mermin, "Topological Theory of Defects in Ordered Media," Reviews of Modern Physics, vol. 51, pp. 591-648, 1979.

[17] R. D. McMichael and M. J. Donahue, "Head to head domain wall structures in thin magnetic strips," Ieee Transactions on Magnetics, vol. 33, pp. 4167-4169, Sep 1997.

[18] Y. Nakatani, A. Thiaville, and J. Miltat, "Head-to-head domain walls in soft nano-strips: a refined phase diagram," Journal of Magnetism and Magnetic Materials, vol. 290, pp. 750-753, Apr 2005.

[19] G. S. D. Beach, M. Tsoi, and J. L. Erskine, "Current-induced domain wall motion," Journal of Magnetism and Magnetic Materials, vol. 320, pp. 1272-1281, Apr 2008.

[20] M. J. Donahue and D. G. Porter, "OOMMF User's Guide, Version 1.0 Interagency Report NISTIR 6376 (National Institute of Standards and Technology, Gaithersburg, MD)," 1999.

[21] E. R. Lewis, D. Petit, A. V. Jausovec, L. O'Brien, D. E. Read, H. T. Zeng, et al., "Measuring Domain Wall Fidelity Lengths Using a Chirality Filter," Physical Review Letters, vol. 102, Feb 62009. 
[22] S. Moretti, V. Raposo, and E. Martinez, "Influence of Joule heating on current-induced domain wall depinning," Journal of Applied Physics, vol. 119, p. 213902, 2016.

[23] T. Shinjo, T. Okuno, R. Hassdorf, K. Shigeto, and T. Ono, "Magnetic vortex core observation in circular dots of permalloy," Science, vol. 289, pp. 930-932, Aug 112000.

[24] A. Vansteenkiste and B. Van de Wiele, "MUMAX: A new high-performance micromagnetic simulation tool," Journal of Magnetism and Magnetic Materials, vol. 323, pp. 2585-2591, Nov 2011.

[25] A. Vansteenkiste, J. Leliaert, M. Dvornik, M. Helsen, F. Garcia-Sanchez, and B. Van Waeyenberge, "The design and verification of MuMax3," Aip Advances, vol. 4, Oct 2014. 


\section{Chapter 4}

\section{Programmable Logic via Gate Controlled Motion of Domain Wall in Magnetic Network Structure}

Spintronics which utilizes the spin as well as charge of an electron for data storage applications offers a non-volatile alternative to the conventional semiconductor based technologies. The binary state of an electron spin can be efficiently employed as logic bits and provide a device which has high endurance, is low-power and has high operating speed. Many proposals exist for spin-based or magnetic logic devices [1-10]. Magnetic logic based on the network called magnetic cellular automata was proposed by Imre et al. [6]. The researchers showed that the ferromagnetic islands which are magnetostatically coupled, perform the logic operation by adding up the stray fields at the nodal dots that have different threshold switching fields. However, the device is prone to fabrication related defects since the magnetostatic coupling is weaker compared to the demagnetizing field. In another study, programmable magnetic tunnel junctions $[4,11]$ based logic devices were proposed, but the current density required is very high. Allwood et al. [5] proposed domain wall (DW) based logic operation in patterned thin films using device design and rotating magnetic field. They employed four different structures for four different operations. However, a logic which is programmable at run-time would simplify the device architecture by removing the interconnects and potentially increase the computation speed. Semiconductor based logic schemes exist demonstrating the programmability by combining magnetism and semiconductor based technologies [12-14]. The logic operation in these devices is achieved via magnetic field controlled motion of charge carriers in $\mathrm{p}-\mathrm{n}$ junction diodes. 
In this chapter, we present a prototype of an all magnetic programmable logic structure capable of performing all basic logic operations in the same structure. The device functions by exploiting the deterministic trajectory of DW in asymmetric branch structure which was discussed in chapter 3 . The programmability of the device is achieved by using a currentcontrolled magnetic gate, which generates a local Oersted field. The transverse charge distribution of DW with certain chirality allows it's trajectory to be manipulated by the local Oersted field generated using current carrying conductor. The DW transformation from vortex to transverse configuration close to the output branch plays a pivotal role in governing the DW chirality and hence the final output. Two universal logic operations NAND and NOR can be obtained by simply switching the current direction in the magnetic gate. At an instant, both the output and its compliment are obtained. The logic functionalities have been demonstrated using magnetic force microscopy (MFM) imaging and magnetoresistance measurements.

\subsection{Device design}

Figure 4-1 (a) shows the schematic of the programmable logic device. The device primarily comprises of two orthogonal nanowires and a half-ring or "U-shaped" branch. The half-ring is displaced by an amount $\mathrm{d}=300 \mathrm{~nm}$ along the $+y$-direction. This makes the structure asymmetric or "pull-up" (PU) which was discussed in chapter 3 section 3.6. The magnetization directions of the two input nanowires correspond to two input bits: the magnetization of the transverse nanowire is input 1 and the magnetization direction of the horizontal nanowire is input 2. The final magnetization direction of the individual branches of the half-ring correspond to the output state. The directions of the magnetization and their corresponding bits are shown in the inset of Figure 4-1 (a). The clocking in the device is achieved via global in-plane magnetic field. The device is clocked by an alternating magnetic field. For each clock cycle, the initial output is pre-set to the logical state of input 2. Logic " 1 " is defined as magnetization 
(a)
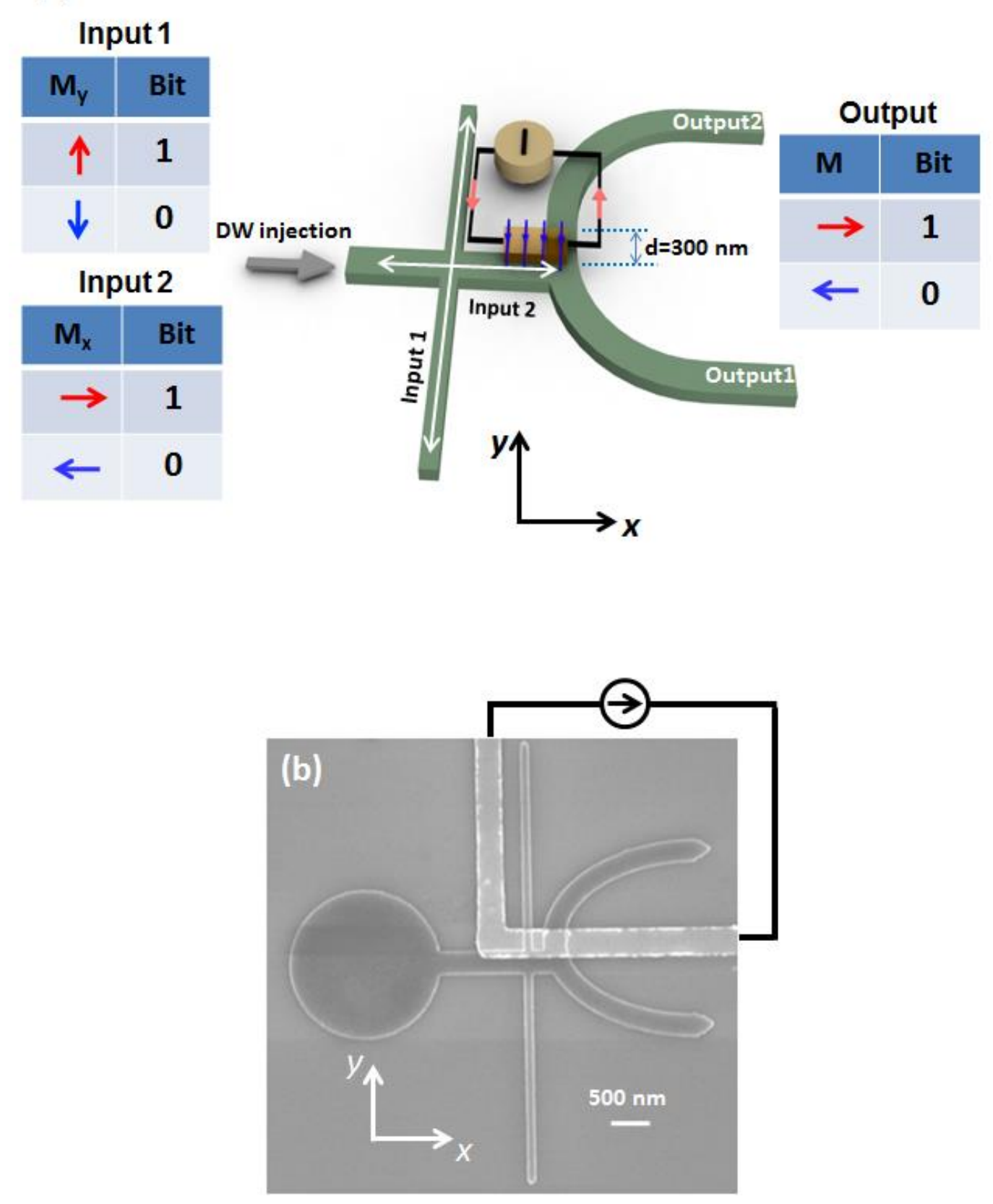

Figure 4-1 (a) Schematic depiction of an all-magnetic programmable domain wall logic. The magnetization orientations along the vertical and horizontal nanowires are considered as input 1 and input 2, respectively. The output is read at the magnetization direction of the upper halfring and lower half-ring. The half-ring is asymmetric with respect to the horizontal nanowire as it is displaced towards $+y$-direction. A non-magnetic metallic strip line is placed at the bifurcation which generates Oersted field when current is flow through it. The strip line acts as a magnetic gate to select between different logic operations. Also shown are the binary representations of the input and output according to the magnetisation direction of the ferromagnetic elements. (b) Scanning electron microscopy (SEM) image of the fabricated device with electrical connection for the metallic stripline. 
pointing in the positive direction along the coordinate axis $(+x$ or $+y)$ and logic " 0 " is defined as the magnetization pointing in the negative direction along the coordinate axis $(-x$ or $-y)$. The output can be programmed by flowing current through the metallic stripline which is patterned on top of the structure at the intersection between the horizontal nanowire and the half-ring. The current flowing through the metallic stripline generates a localized Oersted field and this acts as a magnetic gate controlling the trajectory of the DW. By modulating the polarity of the magnetic gate, multiple logic operations are possible i.e. NAND, NOR, AND, OR, NOT and COPY.

Figure 4-1 (b) shows the scanning electron microscopy (SEM) image of the fabricated device. The thin film is composed of $\mathrm{Ta}(5 \mathrm{~nm}) / \mathrm{Ni}_{80} \mathrm{Fe}_{20}(10 \mathrm{~nm}) / \mathrm{Ta}(5 \mathrm{~nm})$ and deposited using magnetron sputtering technique. The device is patterned using electron beam lithography and Ar-ion milling techniques. The width of the nanowire is kept as $300 \mathrm{~nm}$ to stabilize a vortex DW $[15,16]$. A circular pad is used for injection of DW into the horizontal nanowire. The vertical nanowire, $120 \mathrm{~nm}$ wide and $6 \mu \mathrm{m}$ long, is placed $100 \mathrm{~nm}$ away from the bifurcation. The purpose of the vertical nanowire is to transform the vortex DW to a transverse DW, which is explained in more detail later. The metallic stripline of $\operatorname{Cr}(3 \mathrm{~nm}) / \mathrm{Au}(30 \mathrm{~nm})$ is patterned in the second lithography step to overlap with the upper half of the horizontal nanowire. The extent of overlap and its significance is explained in detail later.

\subsection{Device operation with gate open}

We first discuss the device operation when the current flowing through the metallic stripline is zero. The operation would be similar to the PU structure as discussed in chapter 3, section 3.6. Figure 4-2 (a) shows the MFM images of the device under operation. In case I, the horizontal and vertical nanowires are magnetized along the $+x$ and $+y$ directions respectively. Thus initially the contrast of the two output branches is dark and as per our convention the magnetic 


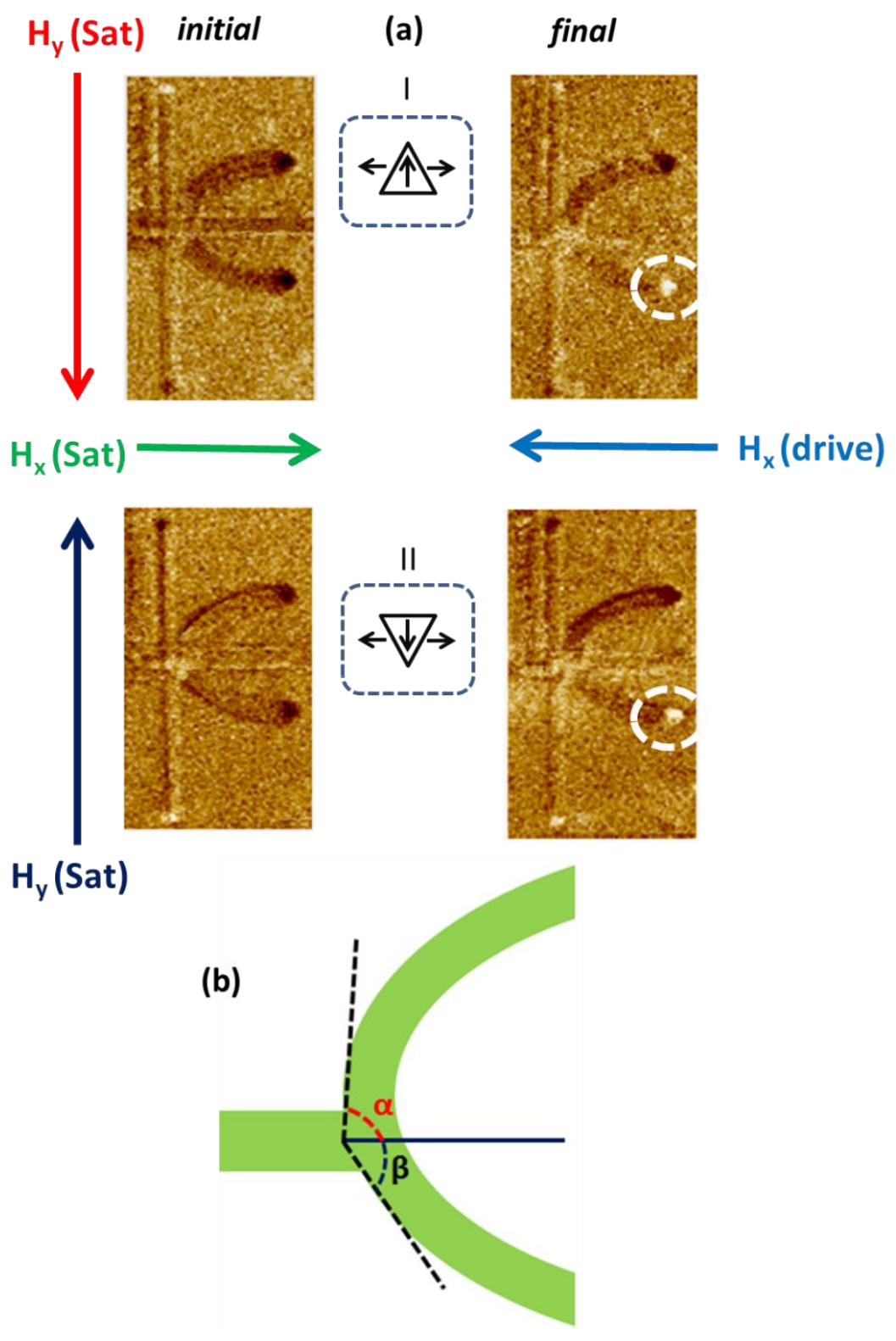

Figure 4-2 (a-I) Magnetic force microscopy (MFM) images of the initial and final configurations of the device when a tail-to-tail DW with "Up" chirality (TT-U) is injected and driven. The TT-U propagates towards the lower half-ring and switches the magnetization direction (a-II) MFM images of the initial and final configurations of the device when a tailto-tail DW with "Down" chirality (TT-D) is injected and driven. TT-D also propagates along the lower half-ring and switches its magnetization direction. (b) Schematic to show the relative angles of the branches along the upper and lower half-rings deviated from the horizontal nanowire when the half-ring is displaced towards $+y$-direction. 
field direction aligns from bright to dark contrast. When the magnetic field is gradually increased in the $-x$-direction, a tail-to-tail (TT) DW is injected with up chirality. In fact, the injected vortex DW is transformed to a transverse DW after propagating across the vertical nanowire. At a field strength of 100 Oe, the DW propagates along the lower branch as shown by the contrast change from dark to bright of the lower branch. In case II, the vertical nanowire is magnetized along the $-y$-direction. Repeating the above exercise, a TT DW with down chirality is injected and driven to the lower branch. Thus in the PU structure the DW always propagates along the lower branch irrespective of its initial chirality.

The deterministic trajectory of the DW in the lower half ring (LHR) can be understood in terms of the potential barrier experienced by the DW at the bifurcation. When there is no offset i.e. the structure is symmetric, the DW experiences similar potential barriers at the upper and lower branch. The trajectory is then governed by the DW chirality as discussed in chapter 3 . When an offset is introduced at the bifurcation, the DW experiences different potential barriers at the upper and lower branches. Figure 4-2 (b) shows a schematic depicting larger angle, $\alpha$, along the upper half ring (UHR) and smaller angle, $\beta$, along the LHR. This indicates that the DW experiences relatively higher potential barrier along the UHR. The steep slope constrains the DW motion along the LHR irrespective of the chirality.

\subsection{Transformation of vortex to transverse domain wall and extent of overlap of gate with the nanowire}

The DW is constrained to propagate along the lower branch due to asymmetry, hence additional control is required to perform the logic operation. A transverse DW has a magnetic charge distribution that can be exploited by an external field and this provides us with additional control. The vertical nanowire placed close to the bifurcation assists in DW transformation from vortex to transverse configuration. Figure 4-3 (a) shows the micromagnetic simulation 


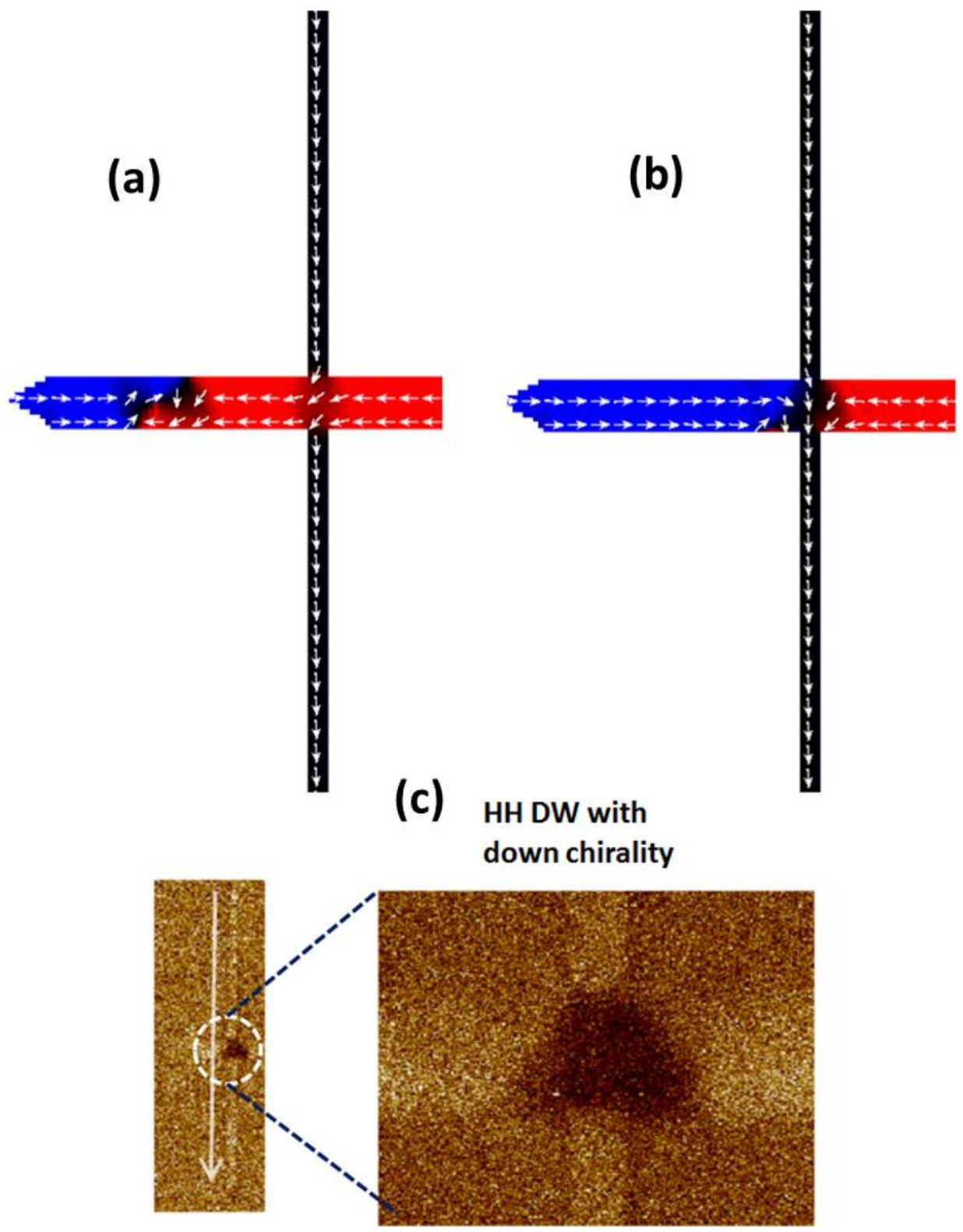

Figure 4-3 (a) Magnetization configuration of a vortex DW relaxed in the nanostructure (b) Magnetization configuration of the transverse DW transformed from the vortex configuration which is pinned under the vertical nanowire when driven by magnetic field (c) MFM image of the head-to-head DW with down chirality that is pinned under the vertical nanowire along with the zoom in image to show the triangular shape of the transverse DW configuration.

results when a clockwise vortex DW approaches a vertical nanowire with magnetization pointing along the $-y$-direction. The vortex transforms to a transverse DW with down chirality as shown in Figure 4-3 (b). Figure 4-3 (c) show the corresponding MFM images. The transverse DW has a characteristic triangular shape and a transversely varying magnetic charge [17]. The 

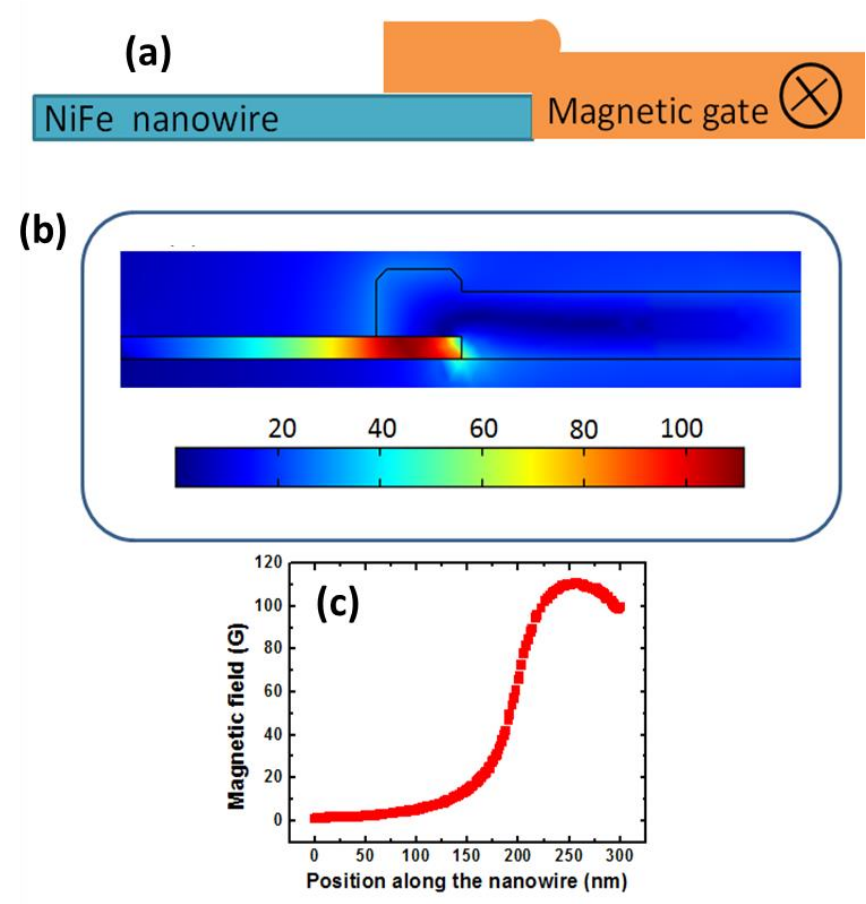

Figure 4-4 (a) Schematic to show the cross-sectional view of the metallic strip line overlapped with the NiFe nanowire. The current flows perpendicular to the plane of paper. (b) Simulated Oersted magnetic field distribution along cross-section of the NiFe due to the current flowing along the gate. (c) Magnetic field strength as a function of position along the NiFe nanowire. The magnetic field is higher beneath the magnetic gate and it drops as we move away from the magnetic gate.

base of the triangle has a higher magnetic charge than the tip. A head-to-head DW (HH-DW) has a positive charge and a TT-DW has a negative charge [17].

The metallic stripline patterned at the upper edge of the horizontal nanowire generates a local Oersted field and this influences the trajectory of DW into the half-ring depending on the polarity and charge distribution of transverse DW. The Oersted field distribution around the magnetic gate is estimated using COMSOL simulations [18]. Figure 4-4 (a) shows the schematic of the magnetic gate overlapping with $25 \%$ of the nanowire. This structure was modelled in COMSOL as shown in Figure 4-4 (b). The color chart is the transverse magnetic field distribution underneath the gate inside the nanowire. This chart indicates that the nanowire directly underneath the Au pad experiences much larger field than the portion which is outside. Figure 4-4 (c) plots the magnetic field inside the nanowire as a function of position. The field 
is estimated to be $110 \mathrm{G}$ beneath the Au pad and around $10 \mathrm{G}$ at the bottom edge. This field interacts with the magnetic charge of the transverse DW having higher charge concentration along the upper edge. The dual control of selective motion of transverse DW allows the device to perform logic operations within a single structure. By changing the polarity of the current in the magnetic gate, two universal logic operations can be realized.

\subsection{Logic operations - NAND and AND}

Here we discuss in detail the experimental verification of the NAND and AND logic operations. A current of $6 \mathrm{~mA}$ is passed from B to A through the stripline as shown in Figure 4-5 (a). The Oersted field, which curls around the metallic line on top of the horizontal nanowire would attract the negative magnetic charge and repel positive magnetic charge.

Inputs " 0 " and "0". The MFM image in Figure 4-5 (b)-I shows the initial magnetization configuration when the horizontal and vertical nanowires are magnetized along the $-x$ and $-y$ directions respectively. This represents that logic level of " 0 " on both the inputs. When a magnetic field of 100 Oe is applied along the $+x$-direction, a HH-DW is injected into the horizontal nanowire. When it crosses the vertical nanowire magnetized along the $-y$-direction, the HH-DW adopts a down chirality. The positive charge is concentrated at the base which is away from the influence of metallic stripline. The DW is primarily influenced by the symmetry of the structure and propagates along the LHR. This is observed by a change in contrast along the lower branch from bright to dark. Now the magnetization in the lower branch points along the $+\mathrm{x}$-direction while that in the upper branch is still in the $-x$-direction. Thus the output of the NAND gate when input is " 0 ", " 0 " is " 1 " along the lower branch. The complimentary output which is " 0 " of the AND gate is present at the upper branch. 
Inputs "0" and "1". Under this input combination, input 2 i.e. the horizontal nanowire is magnetized along the $+x$-direction, while the vertical nanowire remains magnetized in the $-y$ direction. Figure 4-5 (b)-II shows the initial MFM image. The input 2 resets the output branches to show a dark contrast. Application of linear magnetic field along the $-x$-direction, nucleates and drives a TT-DW with down chirality along the upper branch as shown by the switching of the contrast of the UHR from dark to bright, while the LHR remains unchanged. The TT-D DW has negative magnetic charge concentrated at the base facing the stripline, thus it is strongly influenced by the gate magnetic field. The gate attracts the DW towards the UHR against the force of asymmetry. Without the presence of the gate current the DW would propagate along the LHR as dictated by the asymmetry. Thus the input combination " 0 ", "1" still results in UHR having output "0" which is the output of the AND gate and the LHR having output " 1 " which is the output of a NAND gate. The minimum current required by the gate to influence the DW trajectory and overcome the potential barrier due to asymmetry was estimated as $5 \times 10^{11} \mathrm{~A} / \mathrm{m}^{2}$.

Inputs "1" and " 0 ". In this input combination, the vertical nanowire is magnetized along the $+y$-direction while the horizontal nanowire is magnetized along the $-x$-direction. As the linear magnetic field is increased along the $+x$-direction, a HH-DW with up chirality is injected and driven along the LHR as shown by the final MFM image in Figure 4-5 (b)-III. The magnetization in the LHR points along the $+x$-direction while that of the UHR remains unchanged along the $-x$-direction. The HH-U DW has positive charge concentrated along the base facing the stripline and is strongly influenced by the gate Oersted field, which repels it to the LHR. In this case both the asymmetry and gate field assist to push the DW to the LHR. Thus for input combination " 1 " and " 0 " also the UHR has output " 0 " corresponding to the AND gate while the LHR has output "1" corresponding to the NAND gate. 



Figure 4-5 (a) Schematic of the device with orientation of the Oersted field and the current. The current is flown from B to A through the metallic strip. The red arrow represents the current direction and the blue lines represent the direction of current induced Oersted field. The Oersted field generated at the magnetic gate attracts the TT DW and repels the HH DW in this configuration (b) MFM images of the initial and final configurations of the structure for four different combinations of the magnetisation directions of chirality selector $(y)$ and the nanowire $(\boldsymbol{x})$. For ("0" \& "1") combination, a TT-D is injected which is attracted towards the upper halfring as the Oersted field can overcome the potential barrier generated by the asymmetry at the 
bifurcation. In the all other three cases, the DWs are driven towards the lower half-ring as the asymmetry dominates over the Oersted field at the bifurcation.

Inputs "1" and "1". For this input combination, both the vertical and horizontal nanowires are magnetized along the positive directions ( $+y$ and $+x$-directions). As magnetic field is increased along the $-x$-direction, a TT-DW with up chirality is injected and driven along the LHR as shown by the final MFM image in Figure 4-5 (b)-IV. The contrast of the LHR changes from dark to bright and the final magnetization of the LHR points along the $-x$-direction while that of the UHR remains along the $+x$-direction. The TT-U DW has negative charge concentrated along the base and is away from the influence of the gate Oersted field. It is weakly attracted by the gate which is not able to overcome the potential barrier created by the asymmetry. Hence, the DW propagates along the LHR under the influence of asymmetry. Thus for the input combination " 1 ", " 1 " the output of the LHR is " 0 " which corresponds to the NAND gate output, while the output of the UHR is " 1 " which corresponds to the AND gate output.

The logical output at the UHR and LHR are summarized in the truth table in Table 1 . The logical outputs for UHR and LHR are complimentary to each other. For current flowing in the stripline from B to A, the LHR corresponds to the NAND logic gate and UHR corresponds to the AND logic gate. Thus in single clock cycle two complimentary logic operations can be realized simultaneously.

\subsection{Logic operations NOT and COPY}

The input 1 was fixed to logic bit " 1 " by magnetizing the vertical nanowire along the $+y$ direction and current was flown in the gate from B to A. When the structure is saturated along the $-x$-direction, which corresponds to bit " 0 " for input 2 , the DW propagation leads to the switching of the LHR resulting in logical output "1" as shown in the final MFM image of Figure 4-5 (b)-III. When the structure is saturated along the $+x$-direction which corresponds to 
bit " 1 " for input 2, the final output of LHR reveals output " 0 " as shown in Figure 4-5 (b)-IV. Thus the output bit UHR always follows the input bit while LHR is always complimentary of the input bit. The truth table formed in Table 2 shows the structure operates as logical NOT gate when the output is read at the LHR while a COPY operation is obtained at the UHR.

Table 1

\begin{tabular}{|c|c|c|c|c|c|c|}
\hline & & & \multicolumn{2}{|c|}{ AND } & \multicolumn{2}{|c|}{ NAND } \\
\hline $\begin{array}{l}\text { My } \\
\text { (Input 1) }\end{array}$ & & $\begin{array}{c}\text { Mx } \\
\text { (Input 2) }\end{array}$ & $\begin{array}{l}\mathrm{M} \text { (UHR) } \\
\text { (Output 1 }\end{array}$ & & $\begin{array}{c}\text { M(LHR) } \\
\text { (Output 2) }\end{array}$ & \\
\hline$\downarrow$ & 0 & $\leftarrow 0$ & $\leftarrow$ & 0 & $\rightarrow$ & 1 \\
\hline$\downarrow$ & 0 & $\rightarrow \quad 1$ & $\leftarrow$ & 0 & $\rightarrow$ & 1 \\
\hline$\uparrow$ & 1 & $\leftarrow 0$ & $\leftarrow$ & 0 & $\rightarrow$ & 1 \\
\hline$\uparrow$ & 1 & $\rightarrow 1$ & $\rightarrow$ & 1 & $\leftarrow$ & 0 \\
\hline
\end{tabular}

Table 4-1 NAND and AND logic operations Truth table formed based on the input and output magnetization orientations of the device which shows AND and NAND functionalities at upper half-ring and lower half-ring, respectively.

Table 2

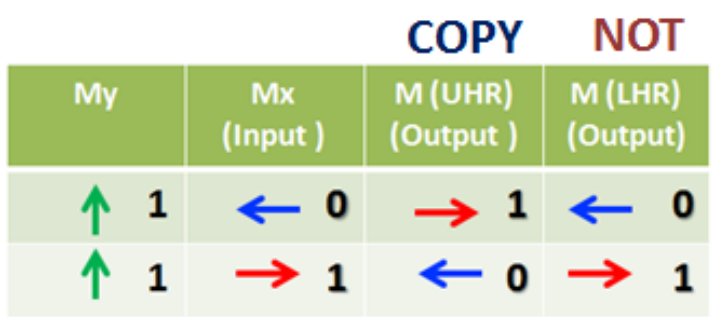

Table 4-2 NOT and COPY logic operations Truth table formed based on the input and output magnetization orientations of the device for single bit logic operation by keeping the chirality selector fixed along $+y$ direction. The results show COPY and NOT functionalities at upper half-ring and lower half-ring, respectively.

\subsection{Logic operations NOR and OR}

When the current flow direction is reversed in the stripline i.e. from A to $\mathrm{B}$, the polarity of the local Oersted field can be switched, as shown in Figure 4-6 (a). This local field would now 
attract the positive magnetic charge but repel the negative magnetic charge. Following the previous discussion of the NAND gate, the magnetic gate has no effect on the DW when the two input bits are same i.e. input 1 and input 2 are " 0 "(" 1 ") and " 0 "(" 1 ") respectively as the charge is concentrated at the lower edge of the nanowire. Thus the output for these input combinations remain same irrespective of the gate polarity as shown in Figure 4-6 (b) I \& IV. The other two input combinations are discussed here. Figure 4-6 (b) II depicts the case when input 1 is logic bit " 0 " and input 2 is logic bit " 1 ". As magnetic field is increased along the $-x$ direction, TT-DW with down chirality is injected. Since the magnetic charge is negative and concentrated near the gate the DW is repelled by the Oersted field. The asymmetry also propels the DW to the LHR. Thus the magnetic contrast of the LHR changes from dark to bright and the magnetization points along the $-x$-direction. The magnetization of the UHR remains along the $+x$-direction. Thus the input combination " 0 ", " 1 " leads to LHR having output " 0 " corresponding to the NOR gate output and the UHR having the output " 1 " corresponding to the OR gate output.

When input 1 is logic bit "1" and input 2 is logic bit "0", a HH-DW with up chirality is injected. Since the positive charge is concentrated at the upper part which is near the metallic stripline, the positive charge or the DW is attracted by the stripline in the UHR. In this case the applied Oerseted field is able to overcome the potential barrier created by the asymmetry. The magnetic contrast in the UHR switches from bright to dark as shown in the final MFM image of Figure 4-6 (b)-III. Thus the input combination "1", "0" leads to the LHR having output " 0 " corresponding to the NOR gate output and the UHR having output "1" corresponding to the OR gate output.

The results for logic operation, where the magnetic gate is programmed with current flowing from A to B are summarized in the truth table, Table 3. The logic output at the LHR corresponds to the NOR gate operation and at the UHR corresponds to the OR gate operation. The 
experimental results show that the device can be programmed to perform both universal logic operations (NAND and NOR) by changing the direction of current flow through the metallic stripline.
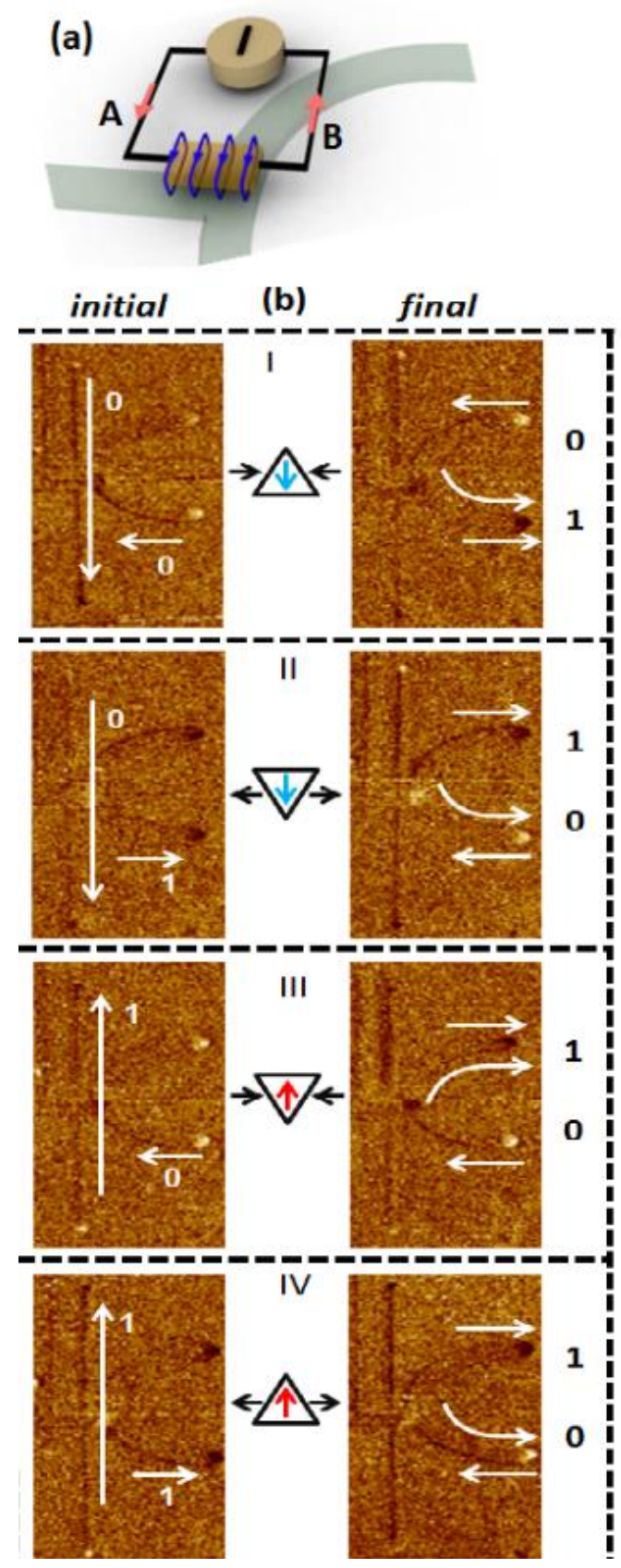

Figure 4-6 (a) Schematic of the device with orientation of the Oersted field when the current is flowing from A to B through the metallic strip. The Oersted field attracts the HH DW and repels the TT DW in this configuration (b) MFM images of the initial and final configurations of the structure for four different combinations of the magnetisation directions of chirality selector $(\boldsymbol{y})$ and the nanowire $(\boldsymbol{x})$. Here, for ("1" \& "0") HH-U is attracted towards the upper 
half-ring as Oersted field overcomes the potential barrier. In the rest of the input combinations, the DW is driven towards the lower half-ring as dictated by asymmetry at the bifurcation.

\section{Table 3}

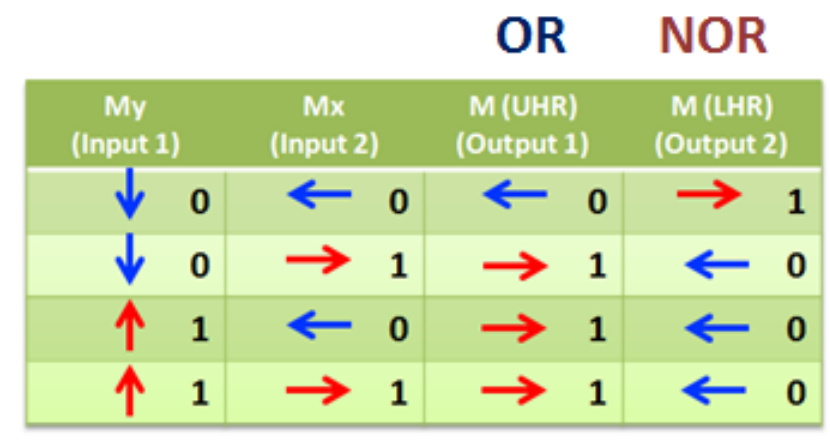

Table 4-3 NOR and OR logic operations Truth table formed based on the input and output magnetization orientations of the device which shows OR and NOR functionalities at upper half-ring and lower half-ring, respectively.

\subsection{Verification of logic functionality using magnetoresistance measurements}

The experimental verification of multiple logic operations in a single structure is also demonstrated using magnetoresistance measurements. As a proof of concept anisotropic magnetoresistance (AMR) detection measurements [19-21] were performed on our sample. As the switching of the individual branches is mediated via DW motion, we have directly probed the presence of DW within the branches. In-situ AMR measurements were performed on the device to test the logic operations by electrical means. The presence or absence of DW within a branch was detected to confirm successful logic operation. Figure 4-7 (a) shows the SEM image of the device with electrical contacts for AMR measurements. A notch is added in each of the branches to pin the DW. The potential difference across each branch (A1 to A2 and B1to B2) was directly measured by placing the DC probes in a four-point probe arrangement. A 


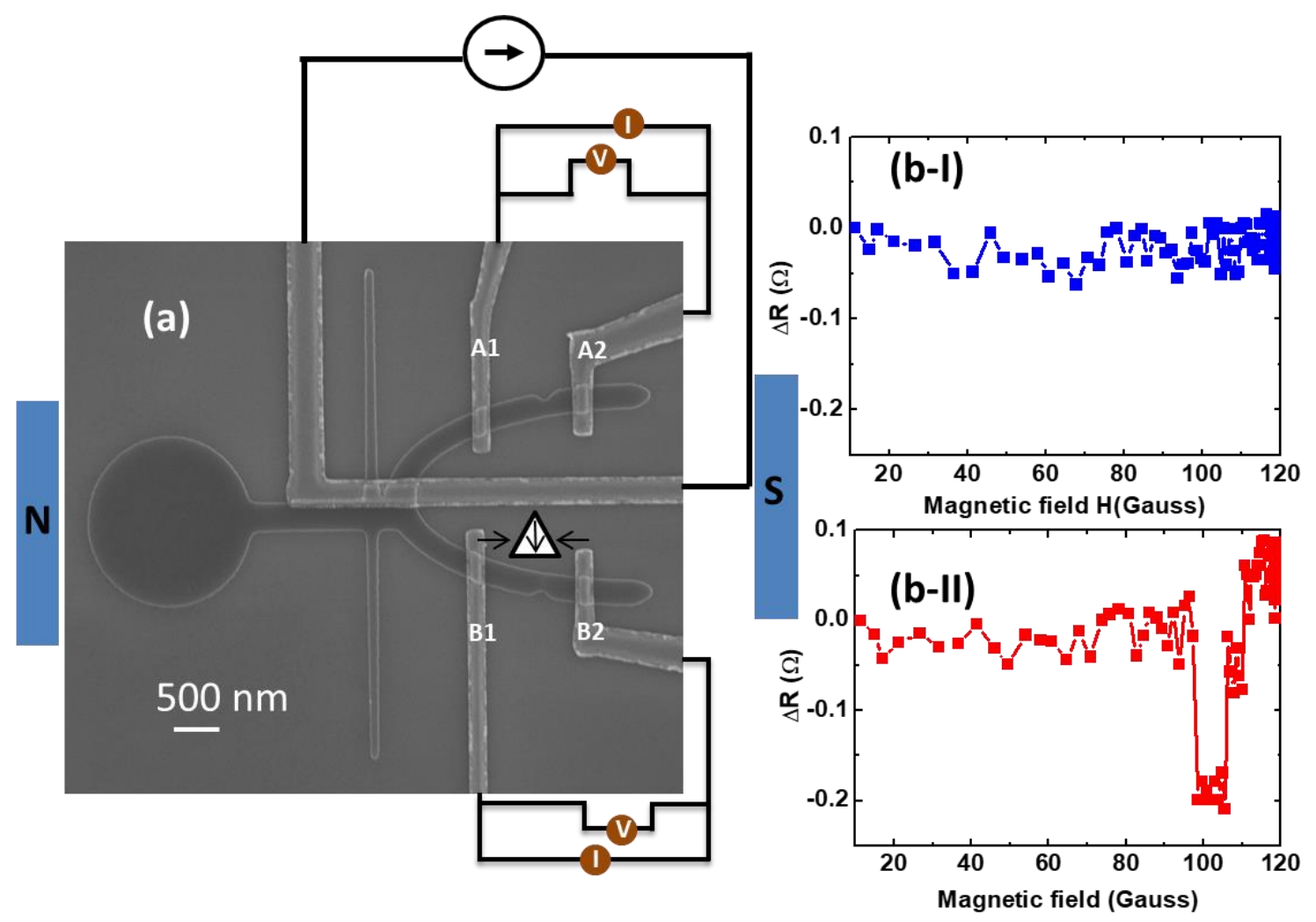

Figure 4-7 (a) SEM image of the device with electrical connections for anisotropic magnetoresistance (AMR) measurements. (b-I\&II) AMR signal of the upper and lower branches during the DW motion through the structure, respectively. A HH-D is injected and driven towards the bifurcation. The DW motion along the lower half-ring can be seen by the change in the magnetoresistance along the lower half-ring. The dip in magnetoresistance shows the pinning and depinning of the DW at the notch structure in the lower half-ring.

constant DC bias of $50 \mu \mathrm{A}$ was applied across the current probes while measuring the voltage drop. The current in the stripline is flown from B to A to obtain NAND gate output in the LHR.

Figure 4-7 (b) shows the in-situ AMR plots for the two output branches as external magnetic field is increased. $\Delta \mathrm{R}=\mathrm{R}(0)-\mathrm{R}(\mathrm{H})$ is the change in magnetoresistance of the individual branches. The initial configuration of the device was with input 1 i.e. the vertical nanowire set to logic bit " 0 " or magnetized along the $-y$-direction, while the input 2 i.e. the horizontal nanowire set to logic bit " 0 " or magnetized along the $-x$-direction. Application of magnetic field along the $+x$-direction injects HH-DW with down chirality. Figure 4-7 (b)-I shows the change in resistance of the UHR which remains constant as magnetic field is gradually 
increased. In the LHR, a drop in resistance appears at a field strength of 95 Oe which signifies the DW reaching the LHR and pinning at the notch. When field is further increased to $110 \mathrm{Oe}$, the resistance reverts back to the original value signifying depinning of the DW from the notch. The change in the magnetoresistance is found to be $0.2 \Omega$ consistent with the DW resistance. This result verifies the NAND gate operation described in section 4.4 for input combination of “0”, “0”.

Figure 4-8 shows the AMR measurements for NOR gate operation i.e. when the current in the stripline flows from A to B. The AMR output in the upper and lower branches are shown for different input combinations indicated on the left of the plots. The branch which receives the DW shows a drop in resistance with field and subsequent DW depinning leads to rise in the resistance. The branch which does not receive the DW shows no change in the resistance. These observations correlate with the MFM results shown in Figure 4-6. There are slight changes in the depinning field for some input combinations, for instance input combination " 0 ", " 1 " show a larger depinning field (close to $120 \mathrm{Oe}$ ) as compared to other input combinations. This could be due to slight stochasticity inherent on account of thermal effects. The AMR measurements presented are electrical validation of the logic operations.

Logic gates realized thus far can be incorporated in actual circuits also. The magnetic signal can be electrically read using magnetic tunnel junctions (MTJ) integrated with output branches. The clocking field can be locally generated by an on-chip inductor in the form of a meander. The change in resistance can also be read as a voltage signal given the current flowing in the device. This voltage signal will be an analog signal which can be further amplified and digitized by passing through a comparator circuit.

To integrate onto a chip, it is necessary to eliminate the need of a magnetic field since it is difficult to scale it to lower dimensions. Another current carrying stripline can be used to generate an Oersted field. The need of a pulsed field can be eliminated by using spin-transfer 
torque (STT). The current driven DW is unidirectional irrespective of the type of the DW. The device is compatible with STT-MRAM and can be solely current controlled.

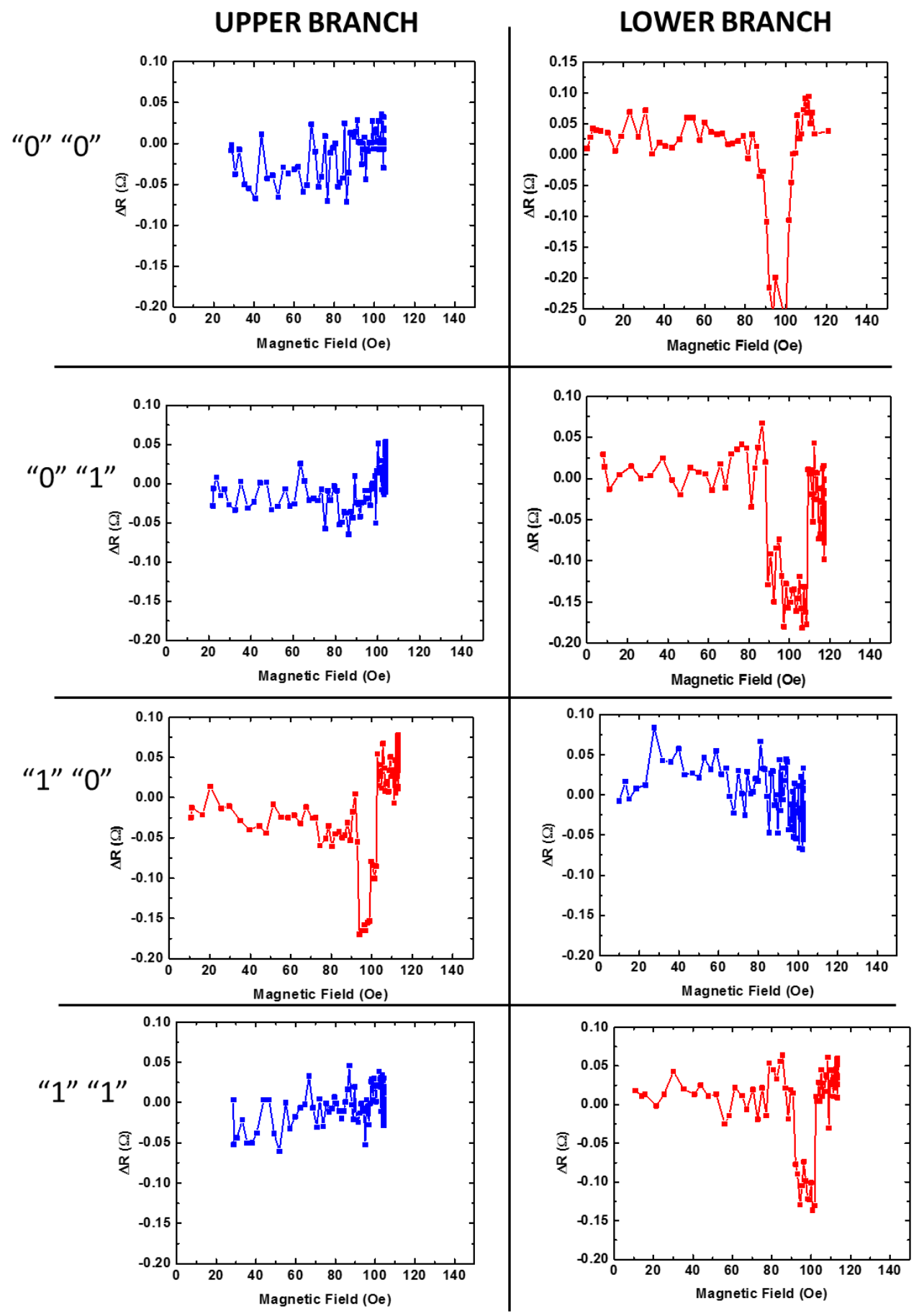

Figure 4-8 AMR measurement results for NOR gate configuration when the current in the stripline flows from A to B. The results are plotted for lower and upper branches respectively for different input combinations indicated on the left. The dip in the magnetoresistance indicates presence of DW pinning and depinning in the respective branch. 


\subsection{Summary}

A prototype of DW based programmable logic device on single structure has been demonstrated. The device is composed of a half-ring or "U"-shaped branch structure which form the output branches; a horizontal nanowire and a vertical nanowire, the magnetization of which forms the input combinations, and a circular pad for nucleation of DW. The information is relayed by DW propagation in the branch structure. The branch is displaced in the vertical direction to create a geometrical asymmetry in the structure which constrains the DW to propagate in the lower branch. The vertical nanowire which serves as one of the input has additional functionality of transforming the vortex to a transverse DW. The transverse magnetic charge distribution in the DW provides additional control over the DW and allows it to be influenced by an external magnetic field. The external magnetic field to control the DW propagation is provided by a current carrying stripline fabricated over the horizontal nanowire such that it only overlaps with $25 \%$ of the nanowire. The partial overlap allows magnetic field control of the DW only when its base is facing the stripline. This is because the base has maximum concentration of the magnetic charge. Thus different chirality can be made to propagate in different branches by optimizing the asymmetry barrier and the gate current. The significant property of the device is that both the output and its complement can be obtained in single operation and two universal logic operations NAND and NOR can be obtained by just reversing the gate current polarity. The logic operations were validated using MFM imaging and electrical magnetoresistance measurements. 


\section{Bibliography}

[1] I. Amlani, A. O. Orlov, G. Toth, G. H. Bernstein, C. S. Lent, and G. L. Snider, "Digital logic gate using quantum-dot cellular automata," Science, vol. 284, pp. 289-291, Apr 91999.

[2] R. P. Cowburn and M. E. Welland, "Room temperature magnetic quantum cellular automata," Science, vol. 287, pp. 1466-1468, Feb 252000.

[3] D. A. Allwood, G. Xiong, M. D. Cooke, C. C. Faulkner, D. Atkinson, N. Vernier, et al., "Submicrometer ferromagnetic NOT gate and shift register," Science, vol. 296, pp. 2003-2006, Jun 142002.

[4] G. Reiss, H. Bruckl, A. Hutten, H. Koop, D. Meyners, A. Thomas, et al., "New materials and applications for magnetic tunnel junctions," Physica Status Solidi a-Applied Research, vol. 201, pp. 1628-1634, Jun 2004.

[5] D. A. Allwood, G. Xiong, C. C. Faulkner, D. Atkinson, D. Petit, and R. P. Cowburn, "Magnetic domain-wall logic," Science, vol. 309, pp. 1688-92, Sep 092005.

[6] A. Imre, G. Csaba, L. Ji, A. Orlov, G. H. Bernstein, and W. Porod, "Majority logic gate for magnetic quantum-dot cellular automata," Science, vol. 311, pp. 205-208, Jan 132006.

[7] P. Xu, K. Xia, C. Z. Gu, L. Tang, H. F. Yang, and J. J. Li, "An all-metallic logic gate based on current-driven domain wall motion," Nature Nanotechnology, vol. 3, pp. 97-100, Feb 2008.

[8] B. Behin-Aein, D. Datta, S. Salahuddin, and S. Datta, "Proposal for an all-spin logic device with built-in memory," Nature Nanotechnology, vol. 5, pp. 266-270, Apr 2010.

[9] A. Lyle, J. Harms, S. Patil, X. F. Yao, D. J. Lilja, and J. P. Wang, "Direct communication between magnetic tunnel junctions for nonvolatile logic fan-out architecture," Applied Physics Letters, vol. 97, Oct 112010.

[10] S. Goolaup, M. Ramu, C. Murapaka, and W. S. Lew, "Transverse domain wall profile for spin logic applications," Sci Rep, vol. 5, p. 9603, Apr 162015.

[11] A. Ney, C. Pampuch, R. Koch, and K. H. Ploog, "Programmable computing with a single magnetoresistive element," Nature, vol. 425, pp. 485-487, Oct 22003.

[12] H. Dery, P. Dalal, L. Cywinski, and L. J. Sham, "Spin-based logic in semiconductors for reconfigurable large-scale circuits," Nature, vol. 447, pp. 573-576, May 312007.

[13] S. Joo, T. Kim, S. H. Shin, J. Y. Lim, J. Hong, J. D. Song, et al., "Magnetic-field-controlled reconfigurable semiconductor logic," Nature, vol. 494, pp. 72-76, Feb 72013.

[14] Z. C. Luo, X. Z. Zhang, C. Y. Xiong, and J. J. Chen, "Silicon-Based Current-Controlled Reconfigurable Magnetoresistance Logic Combined with Non-Volatile Memory," Advanced Functional Materials, vol. 25, pp. 158-166, Jan 72015.

[15] R. D. McMichael and M. J. Donahue, "Head to head domain wall structures in thin magnetic strips," Ieee Transactions on Magnetics, vol. 33, pp. 4167-4169, Sep 1997.

[16] Y. Nakatani, A. Thiaville, and J. Miltat, "Head-to-head domain walls in soft nano-strips: a refined phase diagram," Journal of Magnetism and Magnetic Materials, vol. 290, pp. 750-753, Apr 2005.

[17] H. T. Zeng, D. Petit, L. O'Brien, D. Read, E. R. Lewis, and R. P. Cowburn, "The influence of wire width on the charge distribution of transverse domain walls and their stray field interactions," Journal of Magnetism and Magnetic Materials, vol. 322, pp. 2010-2014, Jul 2010.

[18] "COMSOL ver 4.3 User Guide," ed.

[19] M. Hayashi, L. Thomas, C. Rettner, R. Moriya, and S. S. P. Parkin, "Direct observation of the coherent precession of magnetic domain walls propagating along permalloy nanowires," Nature Physics, vol. 3, pp. 21-25, Jan 2007.

[20] M. Munoz and J. L. Prieto, "Suppression of the intrinsic stochastic pinning of domain walls in magnetic nanostripes," Nature Communications, vol. 2, Nov 2011.

[21] C. Guite, I. S. Kerk, M. C. Sekhar, M. Ramu, S. Goolaup, and W. S. Lew, "All-electrical deterministic single domain wall generation for on-chip applications," Scientific Reports, vol. 4, Dec 122014. 


\section{Chapter 5}

\section{In-plane Current Induced Stochastic Domain Wall Nucleation in Perpendicular Magnetic Anisotropy Nanowires}

Thin films and nanowires exhibiting strong magnetic anisotropy in out-of-plane direction are promising candidates for storage media applications. In the case of conventional in-plane magnetized anisotropy (IMA) nanowires spin-transfer torque (STT) can be used to propagate domain walls (DWs). However, the current density required to drive the DWs is of the order

of $10^{12} \mathrm{~A} / \mathrm{m}^{2}$ or higher $[1,2]$. This may lead to unwanted Joule heating effects. For the case of perpendicular magnetic anisotropy (PMA) nanowires, the threshold current required to manipulate DWs is much lower due to small width of DWs $(\sim 10 \mathrm{~nm})[3,4]$. This is also useful for scaling as it provides larger device density. Hence, understanding DW dynamics in PMA nanowires is crucial for realizing device applications.

$\mathrm{Co} / \mathrm{Ni}$ multilayers have proven to be an attractive system to study domain wall dynamics due to their large perpendicular anisotropy and large spin polarization which can be tuned by varying layer thicknesses $[5,6]$. Also, large DW velocity of the order of $750 \mathrm{~m} / \mathrm{s}$ has been reported in $\mathrm{Co} / \mathrm{Ni}$ multilayers coupled with synthetic antiferromagnets [7]. Conventional techniques of DW injection in such nanowires involves generation of local Oersted field by passing a current through a metallic stripline $[8,9]$. Recently, there have been reports of alternate method of DW nucleation in trilayer stacks by passing an in-plane current. One report suggests spin-orbit torque induced perpendicular switching and chirality dependent DW motion in $\mathrm{Pt} / \mathrm{Co} / \mathrm{AlO}_{\mathrm{x}}$ stack [10], another report suggests Joule heating assisted DW nucleation in $\mathrm{Pt} / \mathrm{CoFeB} / \mathrm{MgO}$ stack [11]. Magnetization reversal in $\mathrm{Co} / \mathrm{Ni}$ multilayer through spin-orbit torque induced by spin Hall effect in heavy metal underlayer has also been reported [12]. This 
method employs an in-plane magnetic field along with an in-plane current to induce magnetization switching. Phung et al. [13] have reported an efficient method of deterministic DW nucleation in $\mathrm{Co} / \mathrm{Ni}$ multilayers using an in-plane current by artificially inducing anisotropy gradient at the injection site.

In this chapter, we explore field induced as well as current induced DW motion in $\mathrm{Co} / \mathrm{Ni}$ PMA nanowires. Hall cross nanostructures were fabricated and probed via anomalous Hall effect (AHE) [14, 15]. Our investigations revealed multiple DW nucleations in lower anisotropy Hall cross junctions by application of in-plane pulsed current without the need of a localized Oersted field. The nucleation process was found to be stochastic with respect to current density and pulse widths. The DW nucleations were attributed to a combination of higher demagnetization energy at the Hall cross junction, STT and Joule heating effect. Further, we have applied this concept of stochastic DW generation and randomly varying Hall voltages to demonstrate a random number generation device.

\subsection{Magnetic Properties of Co/Ni Multilayers}

The existence of strong PMA in Co/Ni multilayers has been attributed to Néel surface anisotropy due to lowered symmetry at the interface [5]. Another reason previously proposed suggests that there is an appropriate number of valence electrons for the Fermi level to be near specific states. These specific states have spin-orbit interaction causing PMA [16]. A positive interface anisotropy contribution should overcome the negative volume anisotropy to sustain PMA in a system.

The objective of the study was to grow Co/Ni multilayers possessing PMA but with rather low anisotropy. Thin film stack comprised of Ta (3)/Pt (3)/Co (x)/[Ni (y)/Co (x) $]_{2} / \mathrm{Pt}(3) / \mathrm{Ta}$ (3), where thicknesses are in $\mathrm{nm}$. The stack repetition was kept as two since single repetition did not yield PMA. The structure was made symmetric to minimize contributions from 

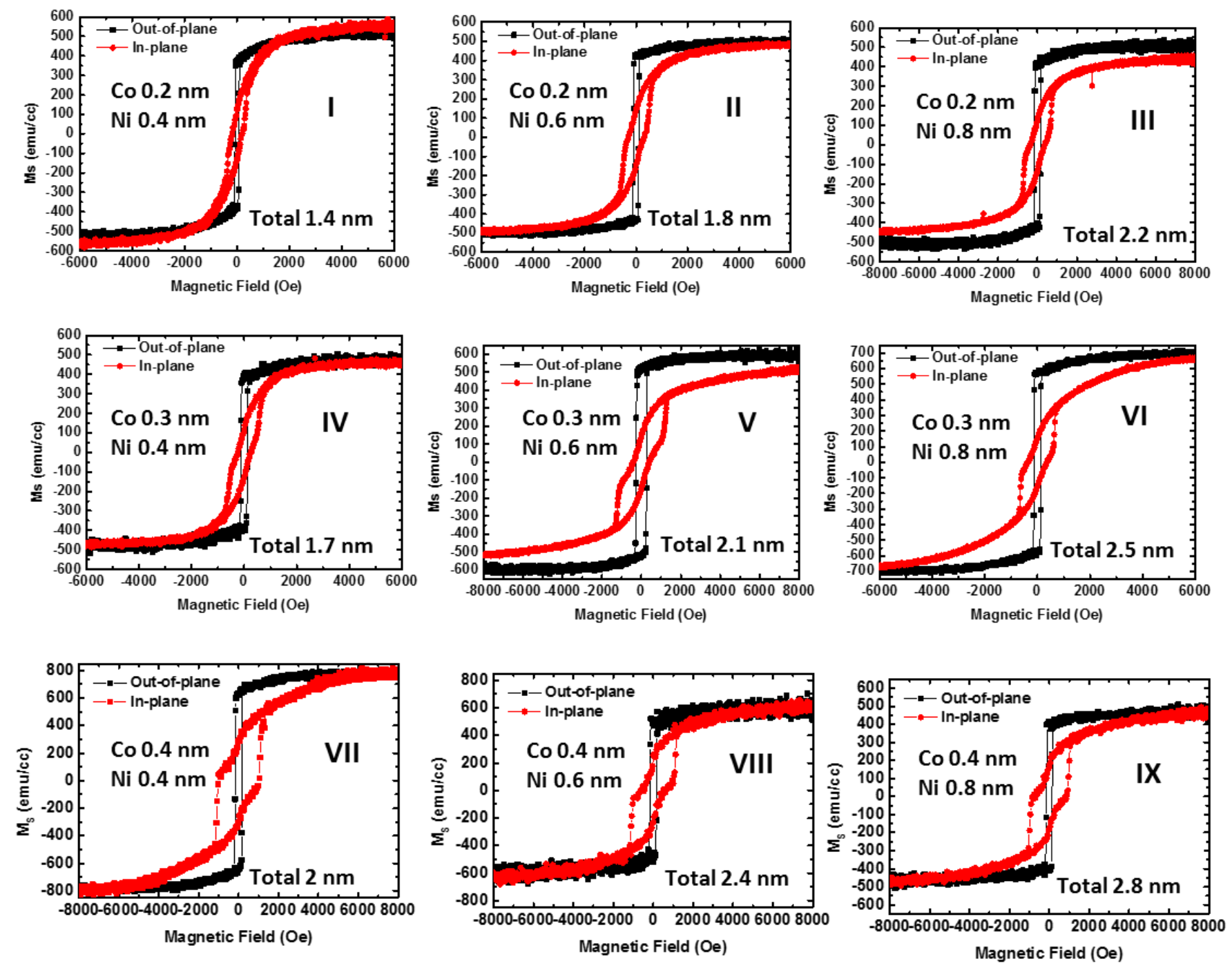

Figure 5-1 M-H loops for $\mathrm{Co} / \mathrm{Ni}$ multilayers in out-of-plane and in-plane field sweep directions. Co thickness varies from $0.2 \mathrm{~nm}$ to $0.4 \mathrm{~nm}$ and Ni thickness varies from $0.4 \mathrm{~nm}$ to $0.8 \mathrm{~nm}$ across different samples

interfacial effects for instance Rashba and spin-orbit torque as discussed subsequently in chapter 6 . Ta was used as the seed layer to provide a smooth interface between the substrate and the magnetic films. Pt was used as the buffer layer to provide strong spin-orbit coupling between the heavy metal and ferromagnetic layer Co, it also provides fcc (111) orientation to favour PMA growth [17]. Co is preferred as the ferromagnetic layer in contact with the heavy metal instead of $\mathrm{Ni}$, since exchange coupling stiffness and Curie temperature of $\mathrm{Ni}$ is 
Table 5-1. Magnetic properties of $\mathrm{Co} / \mathrm{Ni}$ multilayer with respect to magnetic layer thicknesses.

\begin{tabular}{|c|c|c|c|c|c|c|c|}
\hline S. No. & $\begin{array}{c}\text { Co } \\
\mathrm{t}_{\mathrm{CO}} \\
(\mathrm{nm})\end{array}$ & $\begin{array}{c}\mathrm{Ni} \\
\mathrm{t}_{\mathrm{Ni}} \\
(\mathrm{nm})\end{array}$ & $\begin{array}{c}\text { Total } \\
\text { Film T } \\
(\mathrm{nm})\end{array}$ & $\begin{array}{c}\mathrm{M}_{\mathrm{S}} \\
(\mathrm{emu} / \\
\mathrm{cc})\end{array}$ & $\begin{array}{c}\mathrm{M}_{\mathrm{s}} \times \mathrm{T} \\
(\mu \mathrm{emu} \\
\left./ \mathrm{cm}^{2}\right)\end{array}$ & $\mathrm{H}_{\mathrm{K}}(\mathrm{Oe})$ & $\begin{array}{c}\mathrm{K}_{\text {eff }}\left(\times 10^{6}\right. \\
\text { ergs/cc) }\end{array}$ \\
\hline I & 0.2 & 0.4 & 1.4 & 521 & 72.9 & 4000 & 2.2 \\
\hline IV & 0.3 & 0.4 & 1.7 & 475 & 80.7 & 4000 & 1.68 \\
\hline II & 0.2 & 0.6 & 1.8 & 500 & 90 & 4000 & 1.7 \\
\hline VII & 0.4 & 0.4 & 2.0 & 780 & 156 & 6000 & 3.7 \\
\hline V & 0.3 & 0.6 & 2.1 & 550 & 115.5 & 6000 & 2.54 \\
\hline III & 0.2 & 0.8 & 2.2 & 505 & 111.1 & 4000 & 1.52 \\
\hline VIII & 0.4 & 0.6 & 2.4 & 650 & 156 & 5500 & 2.58 \\
\hline VI & 0.3 & 0.8 & 2.5 & 690 & 172.5 & 5000 & 2.45 \\
\hline IX & 0.4 & 0.8 & 2.8 & 460 & 129 & 6000 & 1.88 \\
\hline
\end{tabular}

much lower than Co, leading to disordered spins at the Ni/Pt interface [17]. The thicknesses of Co and Ni layers were varied from $0.2 \mathrm{~nm}$ to $0.4 \mathrm{~nm}$ and $0.4 \mathrm{~nm}$ to $0.8 \mathrm{~nm}$, respectively. Beyond this range the stack was no longer PMA. The thin film stack was deposited using DC magnetron sputtering at a base pressure of $1 \times 10^{-7}$ Torr. The working pressure during deposition was kept as 2 mTorr with $20 \mathrm{sccm}$ Ar flow rate and sputter power of $50 \mathrm{~W}$. The sputter rate calibrated using $\mathrm{X}$-ray reflectivity measurements for $\mathrm{Co}, \mathrm{Ni}$, Ta and $\mathrm{Pt}$ were found to be $0.03,0.04,0.05$ and $0.07 \mathrm{~nm} / \mathrm{s}$ respectively. The magnetic hysteresis curves for the thin films were obtained using alternating gradient force magnetometry (AGFM) technique. Figure 5-1 shows the M-H loops when field is swept in out-of-plane and in-plane directions. The figure shows the plot for 9 samples with different thicknesses of Co and $\mathrm{Ni}$ listed in the plots. The square loop on sweeping the out-of-plane field indicates the existence of PMA in the samples. The anisotropy field, $H_{K}$ is the field at which the film saturates in 


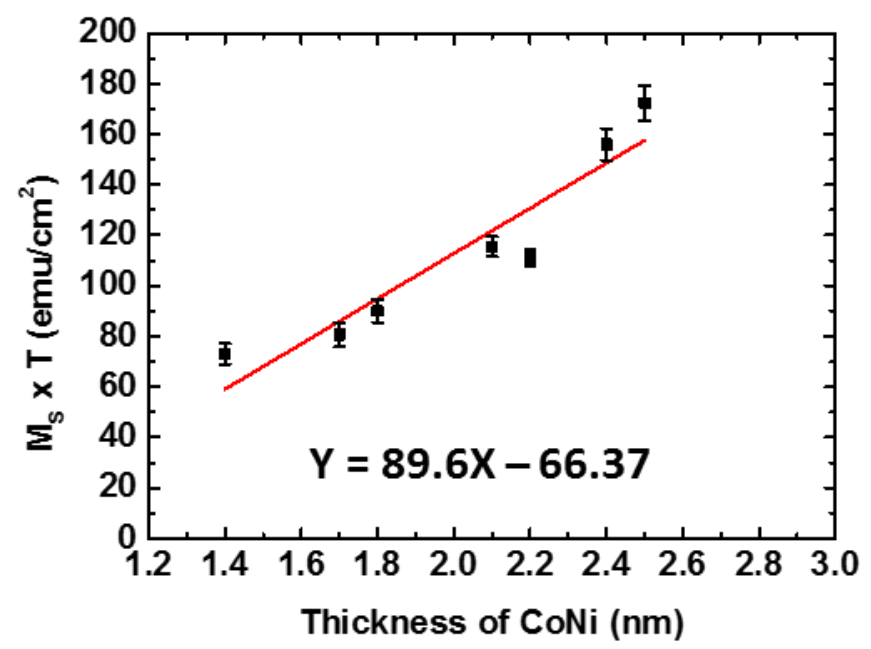

Figure 5-2 Plot of areal magnetization vs thickness of $\mathrm{Co} / \mathrm{Ni}$ multilayers. $\mathrm{X}$-intercept gives dead layer $=0.74 \mathrm{~nm}$.

the in-plane direction and the area under the two curves represent the effective anisotropy, $K_{\text {eff }}=M_{S} H_{K} / 2$. Table 1 shows all the parameters listed for the thin film, the table lists theaverage values. Figure 5-2 shows the plot of $\mathrm{M} \times \mathrm{T}$ vs total thickness of the thin films. The $x$-intercept yields the thickness of magnetic dead layer as $0.74 \mathrm{~nm}$. Slope of the graph gives average magnetization as $896 \mathrm{emu} / \mathrm{cc}$. In this plot the outliers corresponding to samples 7 and 9 have been ignored.

The anisotropy has contributions from surface or interface anisotropy, $\mathrm{K}_{\mathrm{S}}$, and volume anisotropy, $\mathrm{K}_{\mathrm{V}}$. The relation which expresses the anisotropy is as follows:

$$
K_{\text {eff }} \times T=K_{V} \times T+2 K_{S}
$$

where, $\mathrm{T}$ is the total thickness of the magnetic film. For multilayers such as $\mathrm{Co} / \mathrm{Ni}$, the contribution from individual layers need to be considered and the relation is modified as following [16]:

$$
K_{\text {eff }} \times T=K_{V}^{C o} \times t_{C o}+K_{V}^{N i} \times t_{N i}+2 K_{S}
$$


Table 5-2. Total effective anisotropy per unit area with different Co thicknesses keeping Ni thickness fixed at three different values.

\begin{tabular}{|c|c|c|c|}
\hline \multirow{2}{*}{$\begin{array}{c}\text { Co thickness } \\
t_{\text {Co }}(\mathrm{nm})\end{array}$} & \multicolumn{3}{|c|}{$\mathrm{K}_{\text {eff }} \times \mathrm{T}\left(\mathrm{ergs} / \mathrm{cm}^{2}\right)$} \\
\cline { 2 - 4 } & $\mathrm{Ni}=0.4 \mathrm{~nm}$ & $\mathrm{Ni}=0.6 \mathrm{~nm}$ & $\mathrm{Ni}=0.8 \mathrm{~nm}$ \\
\hline 0.2 & 0.145 & 0.18 & 0.22 \\
\hline 0.3 & 0.16 & 0.34 & 0.43 \\
\hline 0.4 & 0.468 & 0.43 & 0.38 \\
\hline
\end{tabular}

Table 5-3. Total effective anisotropy per unit area with different Ni thicknesses keeping Co thickness fixed at three different values.

\begin{tabular}{|c|c|c|c|}
\hline \multirow{2}{*}{$\begin{array}{c}\text { Ni thickness } \\
t_{\mathrm{c}_{0}}(\mathrm{~nm})\end{array}$} & \multicolumn{3}{|c|}{$\mathrm{K}_{\text {eff }} \times \mathrm{T}\left(\mathrm{ergs} / \mathrm{cm}^{2}\right)$} \\
\cline { 2 - 4 } & $\mathrm{Co}_{0}=0.2 \mathrm{~nm}$ & $\mathrm{Co}=0.3 \mathrm{~nm}$ & $\mathrm{Co}_{0}=0.4 \mathrm{~nm}$ \\
\hline 0.4 & 0.145 & 0.16 & 0.468 \\
\hline 0.6 & 0.18 & 0.34 & 0.432 \\
\hline 0.8 & 0.22 & 0.43 & 0.38 \\
\hline
\end{tabular}

where, $\mathrm{K}_{\mathrm{V}}$ is now composed of two contributions from Co and Ni layers and $\mathrm{K}_{\mathrm{S}}$ is the net surface anisotropy contribution by the two layers. To compute the individual anisotropy, one metallic layer thickness is fixed and the other is varied. We have tabulated the contributions in tables 2 and 3 by fixing first Ni thickness and then Co thickness respectively, the table indicates average values. It is expected that $\mathrm{K}_{\mathrm{eff}} \times \mathrm{T}$ should decrease with the thickness of magnetic layer. However, from the results we find that the product is infact increasing in most of the cases except when Co is fixed at $0.4 \mathrm{~nm}$ or $\mathrm{Ni}$ is fixed at $0.8 \mathrm{~nm}$ (in which case the product first increases, then decreases). For other values of thicknesses, the trend is opposite. This is expected for very low thicknesses, if we observe the result in reference [16] where till Co thickness of $0.4 \mathrm{~nm}$, the product infact increases and then decreases. This could be due to requirement of certain minimum Co thickness to initiate crystalline anisotropy. For our case when we increased Co beyond $0.4 \mathrm{~nm}$ to $0.5 \mathrm{~nm}$ the film lost PMA. Figure 5-3 (a) is the plot 
(a)

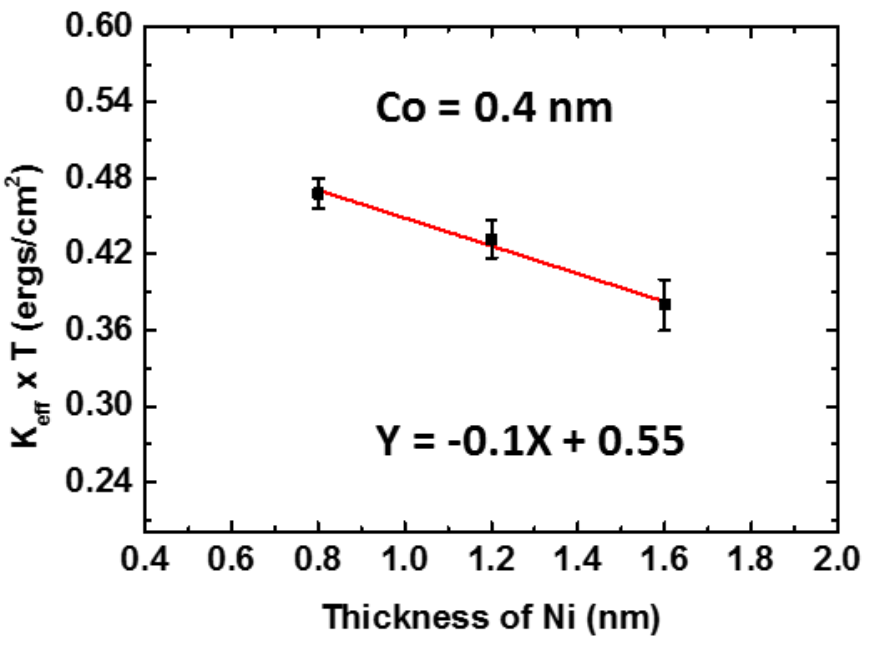

(b)

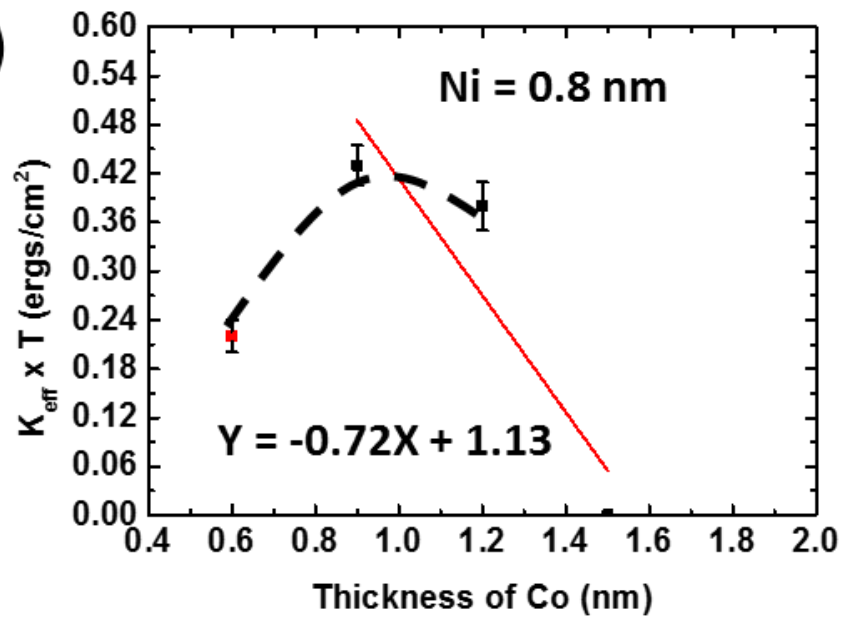

Figure 5-3 (a) Plot of total effective anisotropy energy per unit area vs thickness of Ni with Co thickness fixed at $0.4 \mathrm{~nm}$. (b) Plot of total effective anisotropy energy per unit area vs thickness of Co with Ni thickness fixed at $0.8 \mathrm{~nm}$.

of $\mathrm{K}_{\mathrm{eff}} \times \mathrm{T}$ vs thickness of Ni when Co thickness is $0.4 \mathrm{~nm}$. The linear fit has a slope of -0.1 and intercept of 0.55 . Substituting this value in (2) yields the following results:

$$
2 K_{S}+1.2 \times 10^{-7} K_{V}^{C o}=0.55
$$

where, thickness of Co is taken as $1.2 \mathrm{~nm}$ since there are three Co layers in the stack. Also,

$$
K_{V}^{N i}=-1 \times 10^{6} \mathrm{ergs}^{\mathrm{N}} \mathrm{cc}
$$

Figure 5-3 (b) shows the plot of $\mathrm{K}_{\mathrm{eff}} \times \mathrm{T}$ vs thickness of Co when Ni thickness is fixed at 0.8 $\mathrm{nm}$. It was assumed that $\mathrm{K}_{\text {eff }}$ would be equal to zero at Co thickness equal to $0.5 \mathrm{~nm}$. The linear 
fitting would give the minimum value of volume anisotropy due to Co and surface anisotropy. The slope obtained is -0.72 and intercept is 1.13 . Substituting in (2), the following is obtained:

$$
\begin{gathered}
2 K_{S}+1.6 \times 10^{-7} K_{V}^{N i}=1.13 \\
K_{V}^{C o}=-7.2 \times 10^{6} \mathrm{ergs} / \mathrm{cc}
\end{gathered}
$$

Substituting from (6) in (3) yields $\mathrm{K}_{\mathrm{S}}=0.7 \pm 0.12 \mathrm{ergs} / \mathrm{cm}^{2}$ or $\mathrm{mJ} / \mathrm{m}^{2}$. Substituting from (4) in (5) yields $\mathrm{K}_{\mathrm{S}}$ as $0.645 \pm 0.15 \mathrm{~mJ} / \mathrm{m}^{2}$. Clearly both the values correlate. This value is also comparable to the theoretical values provided in reference [5] for $\mathrm{Co} / \mathrm{Ni}$ multilayers as $0.65 \pm 0.1$ $\mathrm{mJ} / \mathrm{m}^{2}$. Thus the positive value of $\mathrm{K}_{\mathrm{S}}$ compensates the negative contribution from $\mathrm{K}_{\mathrm{V}}$ to sustain PMA. The largest thicknesses of $\mathrm{Co}(0.4 \mathrm{~nm})$ and $\mathrm{Ni}(0.8 \mathrm{~nm})$ were selected for nanowire fabrication since they yielded the least $\mathrm{K}_{\mathrm{eff}} \times \mathrm{T}$. Thus the thin film stack employed for further study was Ta (3)/Pt (3)/Co (0.4)/[Ni (0.8)/Co (0.4)]2/Pt (3)/Ta (3).

\subsection{Field Induced Domain Wall Motion}

To study DW dynamics in the deposited stack, Hall cross nanostructures were fabricated. Figure 5-4 (a) depicts the scanning electron microscopy (SEM) image of the fabricated device along with schematic of the circuit (detailed circuit is shown in Chapter 2 Figure 2-14). A 300nm wide nanowire was patterned using electron beam lithography and Ar ion-milling techniques. Two Ta $(5 \mathrm{~nm}) / \mathrm{Cu}(100 \mathrm{~nm})$ electrodes, labelled A \& B, were formed at both ends of the nanowire to generate local Oersted field and flow current through the nanowire. The contacts were capped with $5 \mathrm{~nm}$ of gold to prevent oxidation. Pulse generator was connected at electrodes A \& B. Two RF probes configured in ground-source-ground formation were used to probe the two electrodes. Hall probe was used to detect the change in magnetization in the nanowire. The Hall resistance $\left(\mathrm{R}_{\text {Hall }}\right)$ which is proportional to the perpendicular magnetization of the Hall cross junction was measured by placing the voltage probes of an external Keithley 
voltmeter on the Hall probes while flowing a constant DC bias of $50 \mu \mathrm{A}$ between electrodes $\mathrm{A}$ \& B.

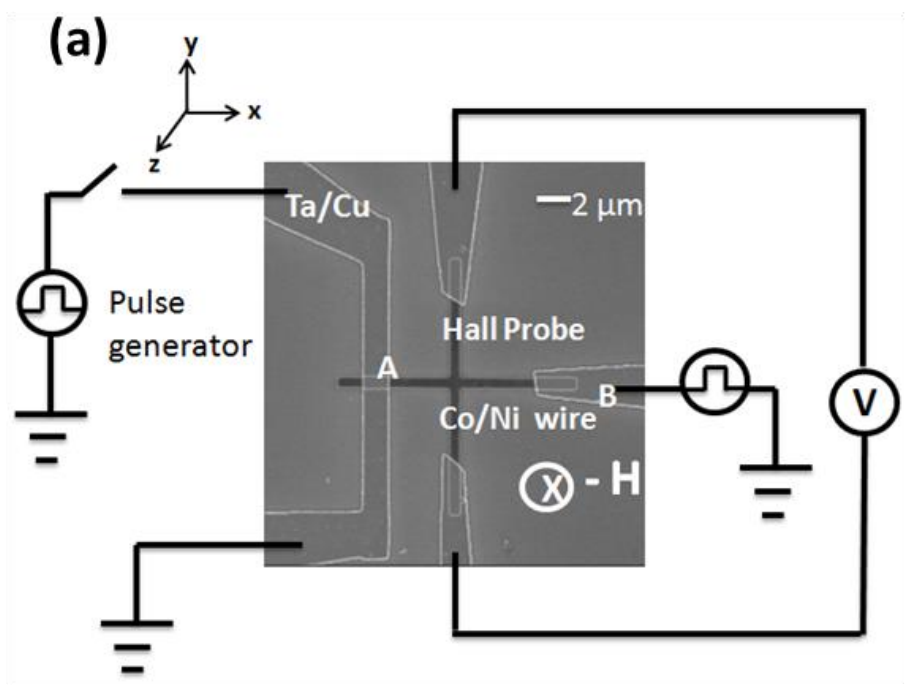

(b)

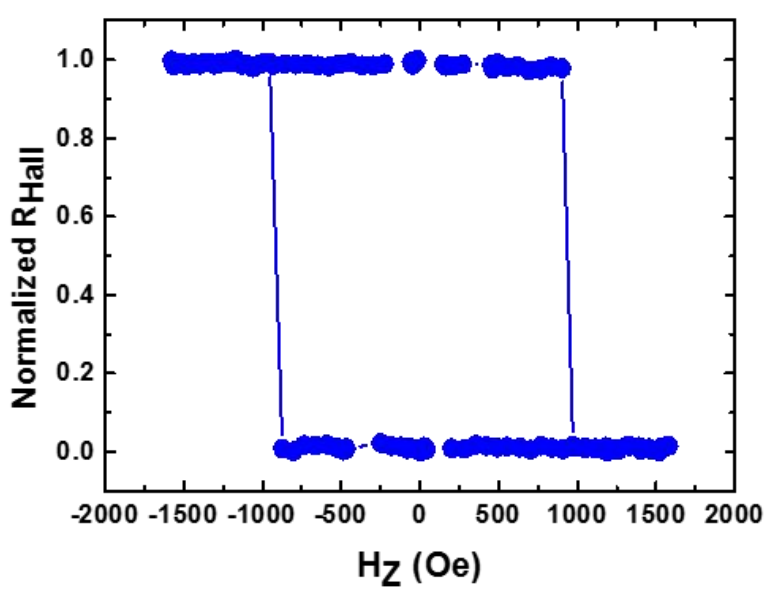

(c)

(d)
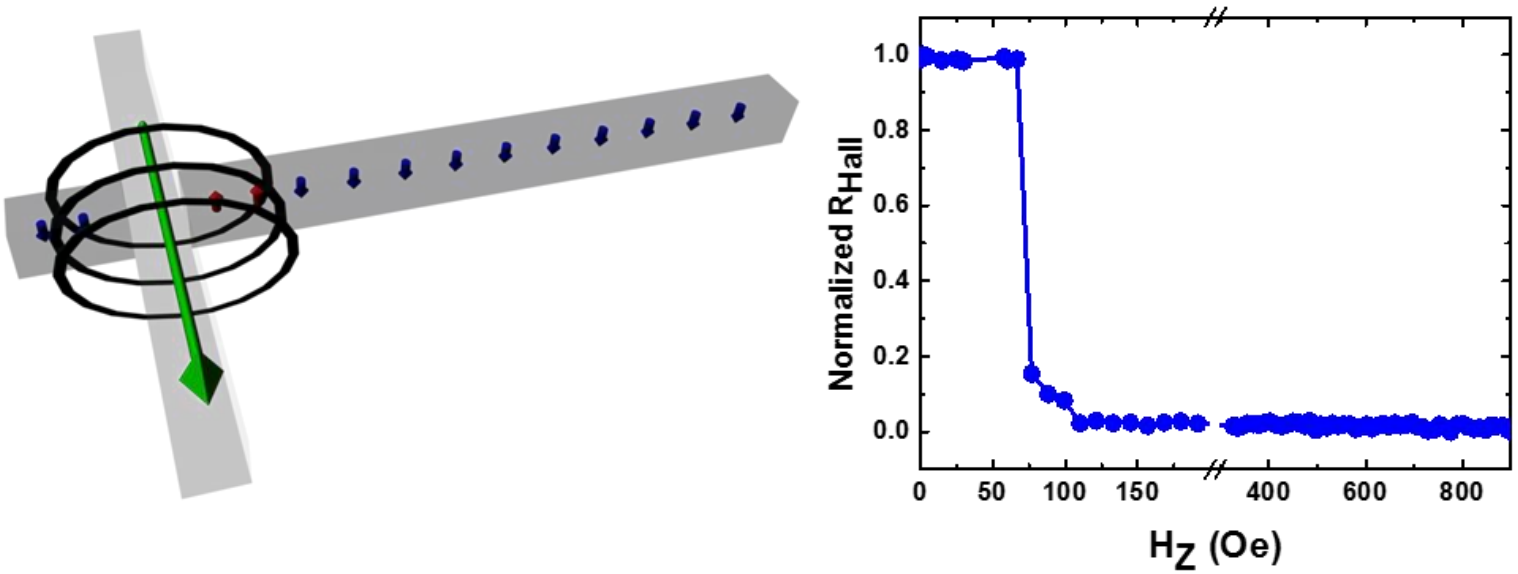

Figure 5-4 (a) Scanning electron microscopy of the fabricated device with external circuit connections. (b) Normalized Hall resistance variation of the nanowire when out-of-plane external field is swept (c) Domain wall nucleation by passing current through the strip line to generate Oersted field. (d) Field induced domain wall driving. The Hall resistance drops at the de-pinning field. 
Figure 5-4(b) shows the normalized $\mathrm{R}_{\text {Hall }}$ measured by sweeping an external magnetic field along the out-of-plane direction. As per our convention the +1 normalized $\mathrm{R}_{\text {Hall }}$ corresponded to field saturation in the $-z$-direction and $0 \mathrm{R}_{\text {Hall }}$ in the $+\mathrm{z}$-direction. A square loop obtained indicated the existence of PMA in the nanowire. The coercivity was found to be $1 \mathrm{kOe}$. DW was injected in the nanowire by first saturating it with a large out-of-plane magnetic field of magnitude $3 \mathrm{kOe}$ along the-z-direction. This was followed by an application of a current pulse ( $85 \mathrm{~mA}, 50 \mathrm{~ns})$ to electrode A. Localized Oersted field is generated near the electrode by the application of current pulse which causes magnetization reversal hence nucleating a DW. The reversal process is depicted by means of a schematic in Figure 5-4(c). This DW was then driven by an external magnetic field. Figure 5-4 (d) shows the plot of normalized $\mathrm{R}_{\text {Hall }}$ obtained by sweeping an external magnetic field along the $+\mathrm{z}$-direction. Two steps were observed in $\mathrm{R}_{\text {Hall }}$ as the field was gradually ramped. The first step at 60 Oe corresponds to DW propagation and pinning at the junction between the Hall probe and the nanowire. The second step at $100 \mathrm{Oe}$ corresponds to DW depinning [18] at the Hall probe and propagation through the nanowire and the Hall probe.

\subsection{Current Induced Domain Wall Motion}

The spin polarized current flowing into the nanowire can transfer its momentum to the localized magnetic moments of a DW leading to its motion. This process is termed spin transfer torque (STT) $[19,20]$. To demonstrate STT process, a DW was nucleated in the nanowire by the process described in the previous section. Next, $10 \mathrm{~ns}$ current pulses were applied from electrode B to A. R Rall was measured and recorded after each pulse. Figure 5-5 shows the variation of normalized $\mathrm{R}_{\text {Hall }}$ with pulse width duration. While the current density was below a certain threshold value no change in the value of $\mathrm{R}_{\text {Hall }}$ was observed. A drop in $\mathrm{R}_{\text {Hall }}$ was observed between pulse width $45 \mathrm{~ns}$ and $50 \mathrm{~ns}$ when the current density reached $7.6 \times 10^{11}$ 
$\mathrm{A} / \mathrm{m}^{2}$. This value corresponds to the intrinsic threshold current density reported previously by Koyama et al. [8].

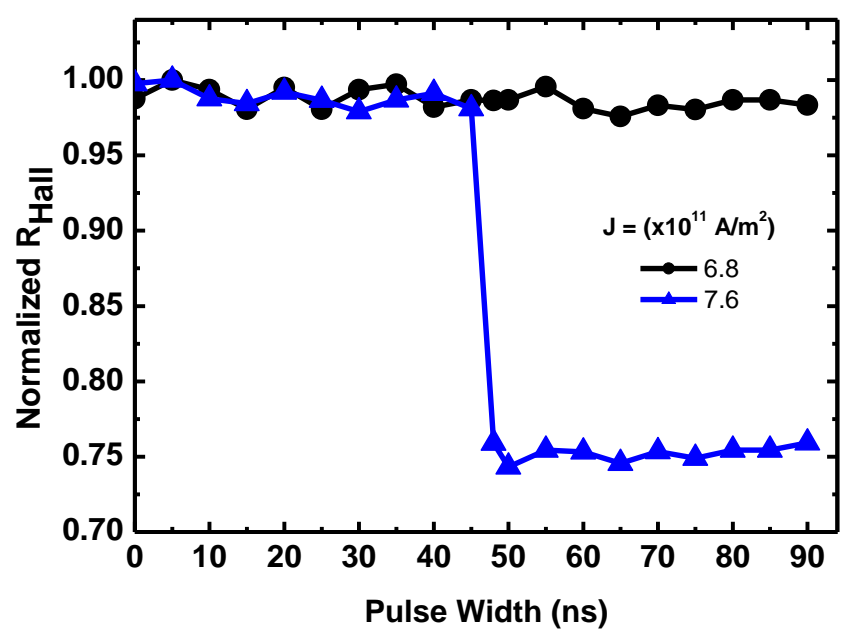

Figure 5-5 Spin transfer torque induced domain wall driving. At threshold current density of $7.6 \times 10^{11} \mathrm{~A} / \mathrm{m}^{2}$ the $\mathrm{R}_{\text {Hall }}$ drops after integrated pulse duration of $50 \mathrm{~ns}$. This indicates domain wall reaching the Hall probe.

\subsection{In-plane Current Induced Domain Wall Nucleation}

A pulsed current was applied between electrodes A \& B to study the effect of in-plane current on the domain wall nucleation process. The nanowire was initially saturated in the $-\mathrm{z}$ direction and pulse current density was varied from $6.8 \times 10^{11} \mathrm{~A} / \mathrm{m}^{2}$ to $1.52 \times 10^{12} \mathrm{~A} / \mathrm{m}^{2}$ while keeping the pulse width constant at $50 \mathrm{~ns}$. After the application of pulse, external magnetic field was gradually increased in the $+\mathrm{z}$-direction and change in the $\mathrm{R}_{\text {Hall }}$ was noted as shown in Figure 56 (a). At low current densities, there was no observable change in the magnitude of $\mathrm{R}_{\text {Hall }}$ indicating no change in the magnetization of the nanowire. However, as the current density increased to $8.7 \times 10^{11} \mathrm{~A} / \mathrm{m}^{2}$, drop in $\mathrm{R}_{\text {Hall }}$ was observed at external field strength of 150 Oe. This indicates magnetization reversal at the Hall cross junction. At the Hall cross junction, the demagnetization energy is higher and the spins at the edge experience lesser exchange 
interaction thereby increasing the probability of DW nucleation in this region. The higher demagnetization energy generates a gradient in anisotropy much like the artificial generation of anisotropy gradient by Phung et al. [13]. As the current density increases, the drop in $\mathrm{R}_{\text {Hall }}$ occurs at lower field strength. At a critical current density corresponding to $1.08 \times 10^{12} \mathrm{~A} / \mathrm{m}^{2}$, the application of current pulse itself reduces the $\mathrm{R}_{\text {Hall }}$ without the need of an external field strength after the application of current pulse.

(a)

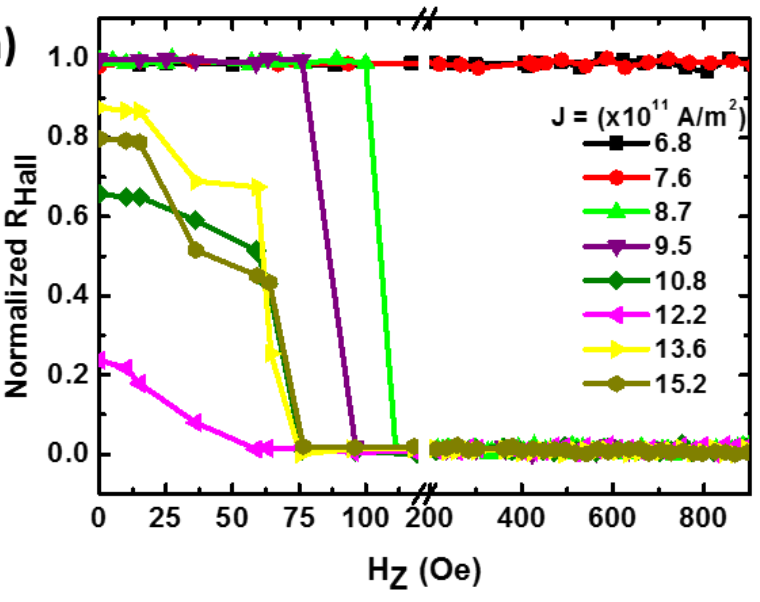

(b)

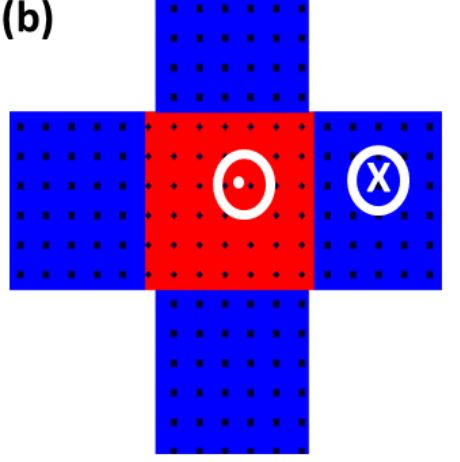

(c)

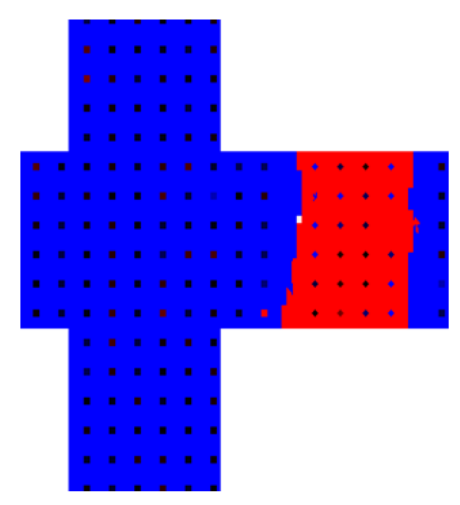

(d)

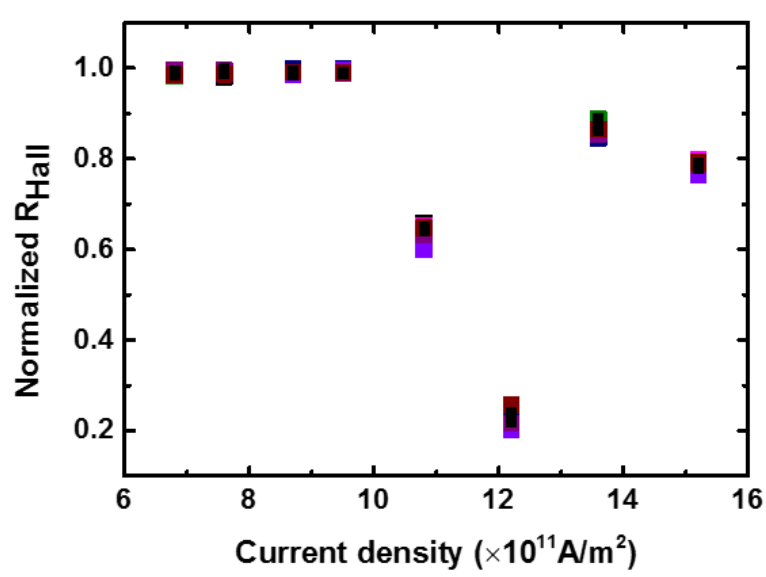

Figure 5-6 Anomalous Hall effect (AHE) measurements on device with Co thickness $=0.4 \mathrm{~nm}$, Ni thickness $=0.8 \mathrm{~nm}$ (sample IX). (a) Normalized R Rall variation with external magnetic field as a function of current density. (b) Simulated magnetization state of the system when DWs are nucleated and the entire magnetic volume at the Hall probe is switched. (c) Simulated magnetization state of the system, when the DWs are driven away from the Hall probe upon application of pulsed current. (d) Statistical distribution of $\mathrm{R}_{\text {Hall }}$ as a function of current density. 
Figure 5-6 (b) depicts the simulated magnetization configuration of the Hall cross junction when four DWs are nucleated at the junction. At lower current density, the area marked 'red' in Figure 5-6 (b), which indicates the reversed magnetized region is less in volume. As magnetic field is increased in the out-of-plane direction, the DWs would move away from each other and the magnetization of the Hall probe would also switch, which would be detected as a drop in the $\mathrm{R}_{\text {Hall. }}$. The external field required to switch the magnetization of the Hall probe would be same for all the current densities, when the magnetization at the junction of nanowire and Hall cross is completely switched. The Hall bar would be switched by two DWs moving in opposite directions along it. The magnetic field corresponding to the DW depinning in the Hall bar has a value of 75 Oe which is same for all the subsequent current densities as shown in Figure 5-6 (a). At the current density of $1.22 \times 10^{11} \mathrm{~A} / \mathrm{m}^{2}$, the $\mathrm{R}_{\text {Hall }}$ reached a minimum value indicating a large switched volume at the Hall cross. When the current density is increased beyond this value, the drop in $\mathrm{R}_{\text {Hall }}$ reduces, indicating the DW displacement away from the junction. The above observation may be described by examining the pinning and de-pinning strength of the DWs at the Hall probe. Previous reports indicate that the DW would experience repulsion at the Hall probe [18] and would precess until the field is strong enough to de-pin the DW from the Hall probe. At low enough current density, the DW is pinned near Hall probe since it is unable to overcome the potential barrier. At this current density $\left(12.2 \times 10^{11} \mathrm{~A} / \mathrm{m}^{2}\right)$, the $\mathrm{R}_{\text {Hall }}$ drops to its minimum value. Upon increasing the current density further, the DWs are de-pinned from the Hall probe and can propagate further into the nanowire as shown by the simulated configuration in Figure 5-6 (c). The DWs are nucleated and driven via STT at this point. Phung et al. [13] report a similar process of DW nucleation which they attribute to the DW extraction from the in-plane magnetized region to the out-of-plane magnetized region by STT. The anisotropy gradient in their structure is provided by ion-irradiation, whereas in our structure there is a naturally generated anisotropy gradient at the four edges of the Hall junction. 
The spins at the edges are canted due to fringing field and are uncompensated, which are driven by STT and responsible for DW nucleation and propagation. The spin configuration at the Hall probe reverts to the original magnetization state and the $\mathrm{R}_{\text {Hall }}$ recovers to the original value after the DWs are de-pinned from it. The plot of normalized $\mathrm{R}_{\text {Hall }}$ with respect to the applied current density is shown in Figure 5-6 (d), these measurements were repeated 20 times. The spread of $\mathrm{R}_{\text {Hall }}$ indicates non-uniformity in reversed magnetization volume, which indicates the presence of stochasticity in the nucleation process. There is a possibility of multiple DW generation contributing to the stochasticity. The Kerr images depicting direct observation of DWs at the Hall cross and nucleation of multiple DWs are shown in the following section.

\subsubsection{Detection of domain wall at the Hall cross by using Kerr imaging}

Kerr microscopy technique is based on the principle of magneto-optical Faraday or Kerr effect. This is discussed in more detail in Chapter 2 section 2.3.3. Figure 5-7 (a) shows the Kerr image of the device magnetized along the -z-direction, Figure 5-7 (b) shows the image after an inplane current is injected. The dark contrast at the Hall junction represents the switched magnetic domains. Further set of trials lead to the nucleation of multiple domains on passing the current as shown in Figure 5-7 (c). The images emphasize the event of DW depinning and propagation away from the Hall junction after nucleation. The Kerr images were taken on 2 $\mu \mathrm{m}$ width wires to get better resolution. Figure 5-7 (d-e) shows the images on $1.5 \mu \mathrm{m}$ width wires. It is worth noting that although the electrical measurements are performed on $300 \mathrm{~nm}$ wide nanowires, the width of the nanowire and shape anisotropy would not play much role in PMA wires hence the nucleation process is similar in larger width nanowires. The thickness of the magnetic layers used for larger width nanowires, used in Kerr imaging, is the same as that of lower width nanowires. Even though the shape anisotropy, which is the ratio of thickness/width of the nanowire, is lower for the larger width nanowires, the interfacial 
anisotropy is strong enough to retain perpendicular anisotropy.The electrical measurements with pulse width modulation for larger width nanowires are shown in Figure 5-9 (d) which is explained later.

(a)

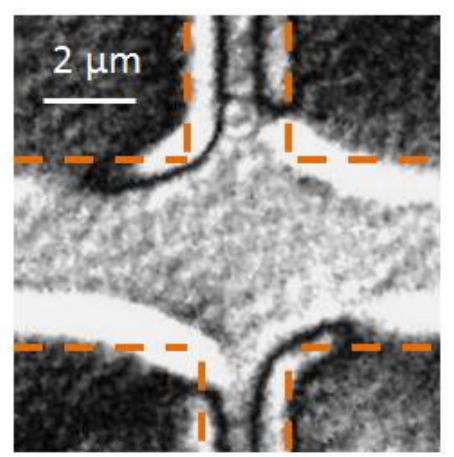

(b)

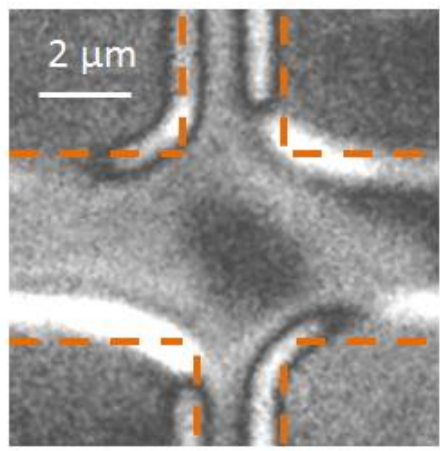

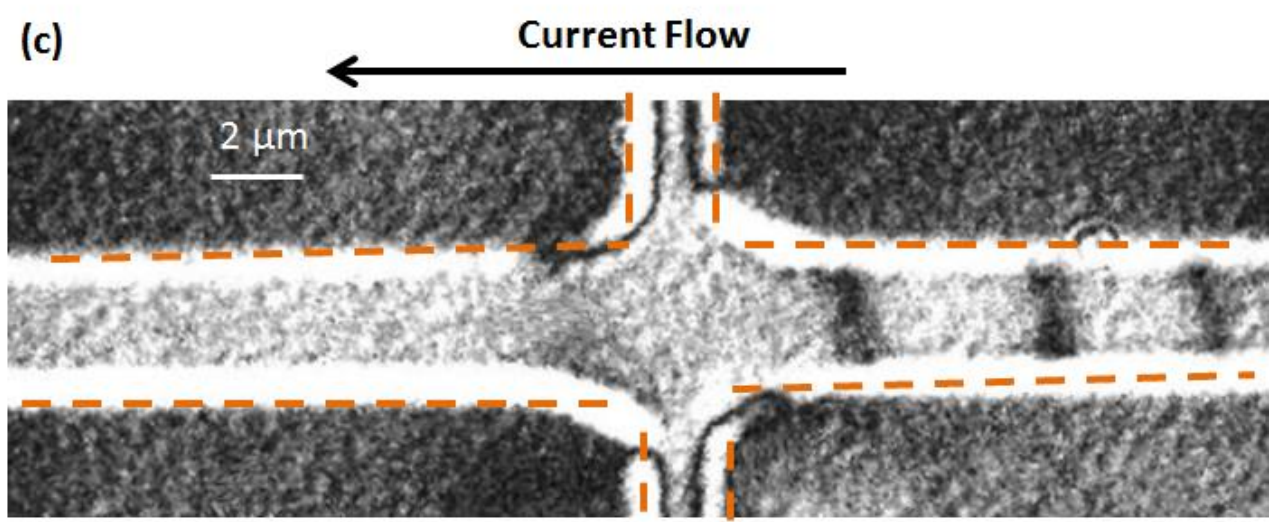

(d)

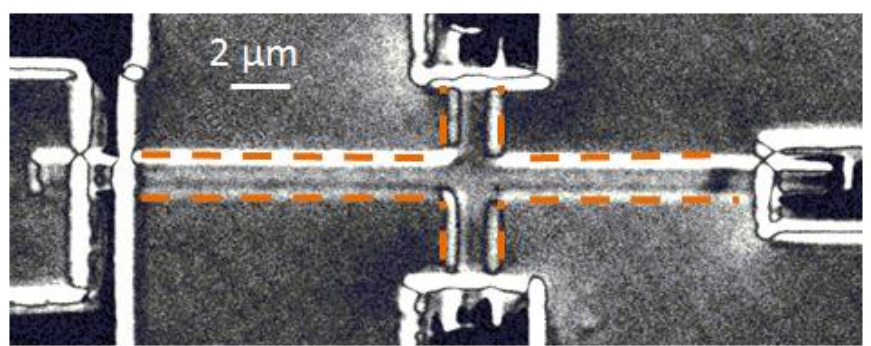

(e)

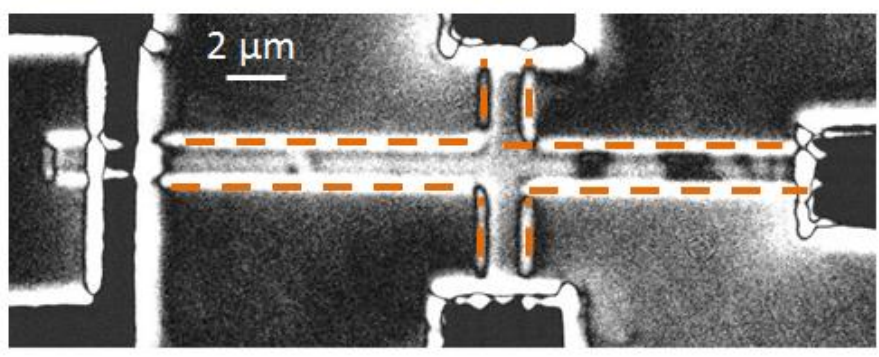

Figure 5-7 (a) Kerr image of device saturated in -z-direction. (b) Injection of in-plane current reverses magnetization in the Hall cross junction and contrast changes to dark. (c) Nucleation and de-pinning of multiple domain walls (DWs) in $2 \mu \mathrm{m}$ nanowire. (d) In-plane current pulse 
application to $1.5 \mu \mathrm{m}$ nanowire nucleates and drives a single DW. (f) Similar magnitude of current pulse nucleates and de-pins multiple DWs in $1.5 \mu \mathrm{m}$ nanowire.

\subsubsection{Back and forth motion of domain walls by spin-transfer torque nucleated at the Hall cross using in-plane current}

To validate the observation of rise in $\mathrm{R}_{\text {Hall }}$ due to depinning of DWs at higher current density, the experiment was extended by preparing the device in the minimum $\mathrm{R}_{\text {Hall }}$ state as that shown in Figure 5-6 (b). The nanowire is initially magnetized in the $-z$-direction, followed by application of current pulses. When the magnetization at the Hall cross changes its direction,

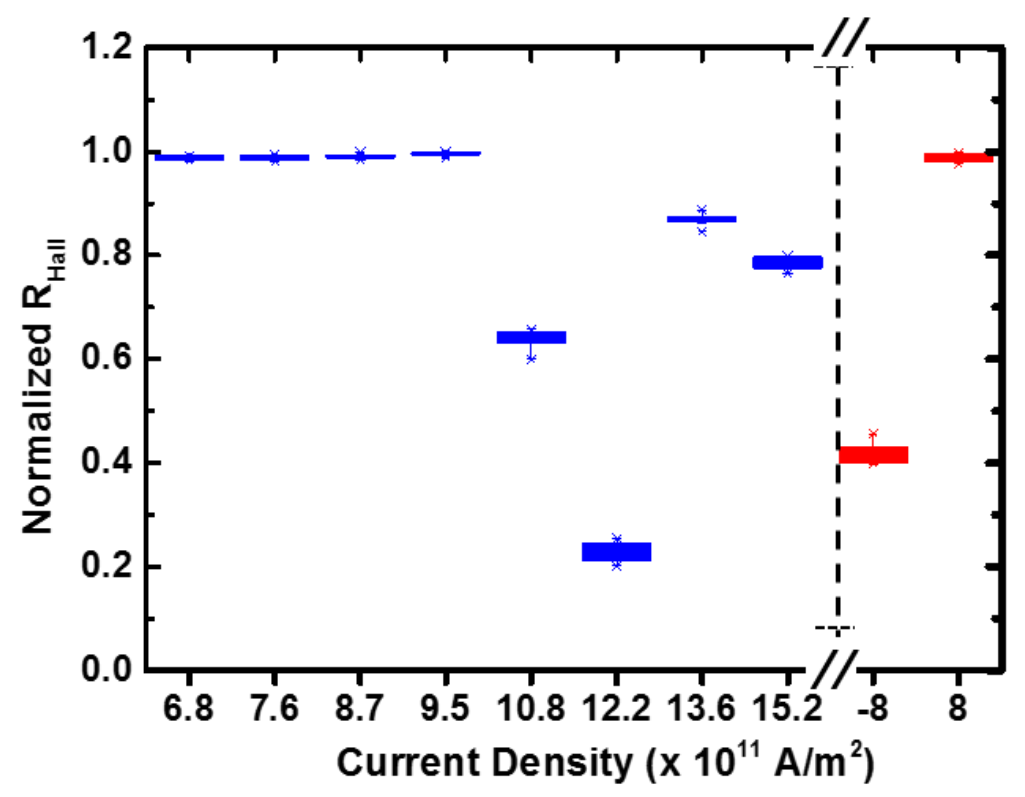

Figure 5-8 Statistical distribution of $\mathrm{R}_{\text {Hall }}$ as a function of current density. X-axis is not scaled to represent DW motion in both directions and compare with previous results.

the $\mathrm{R}_{\text {Hall }}$ reaches a minimum and the magnetization at the Hall cross now points along the $+z-$ direction. On increasing the current density, $\mathrm{R}_{\text {Hall }}$ increases due to displacement of DWs away from Hall cross which is now again magnetized along the $-z$-direction. These observations are shown in Figure 5-8 which is a re-plot of Figure 5-6 (d) with the addition of two more data 
points for which the explanation follows. At this instant, a negative current pulse of magnitude $8 \times 10^{11} \mathrm{~A} / \mathrm{m}^{2}$ was applied to the nanowire. It was observed that $\mathrm{R}_{\text {Hall }}$ approached its minimum value when 20 ns width current pulses were applied, changing the magnetization of the Hall cross back to $+z$-direction. This was followed by the application of current density equal to + $8 \times 10^{11} \mathrm{~A} / \mathrm{m}^{2}$. This pushed the DWs away from the Hall cross and $\mathrm{R}_{\text {Hall }}$ recovered its maximum value when the pulse width reached $25 \mathrm{~ns}$, thereby switching the magnetization of the Hall cross along the $-z$-direction. The motion of the DWs is because of STT.

\subsubsection{Effect of pulse width modulation on domain wall nucleation process}

The effect due to pulse width modulation on the nucleation process was studied keeping the current density constant. Figure 5-9 (a) shows the variation of $\mathrm{R}_{\mathrm{Hall}}$ with respect to the magnetic field which is swept after application of current pulses with different pulse widths. The current density is kept constant at $12.2 \times 10^{11} \mathrm{~A} / \mathrm{m}^{2}$. At low pulse width $=10 \mathrm{~ns}$ the drop in $\mathrm{R}_{\text {Hall }}$ occurs at larger magnetic field strength. This indicates that at lower operating power, current alone is insufficient to cause magnetization reversal and requires assistance from the field. Once DWs are nucleated, the de-pinning of DWs from the Hall junction occurs at around 75 Oe as shown in Figure 5-9 (a). Figure 5-9 (b) shows the plot of normalized $\mathrm{R}_{\text {Hall }}$ after the application of current pulse without applying any magnetic field. The measurements were repeated 20 times to get the distribution. When the pulse width is less than $15 \mathrm{~ns}$, there is no drop in $\mathrm{R}_{\text {Hall }}$ indicating no reversal at the junction. When the pulse width lies in the range of $15 \mathrm{~ns}$ to $50 \mathrm{~ns}$, the $\mathrm{R}_{\text {Hall }}$ varies between 0.8 and 1 indicating no or small reversal at the corners of the junction. Maximum reversal at the Hall junction is observed for pulse width equal to $55 \mathrm{~ns}$ when the $\mathrm{R}_{\text {Hall }}$ falls to its minimum value. The $\mathrm{R}_{\text {Hall }}$ recovers to its maximum value upon increasing the pulse width to $60 \mathrm{~ns}$ indicating depinning of the DWs from the Hall probe like the one observed in Figure 5-6 (c). This also indicates a possibility of multiple DW generation as shown in Figure 
5-9 (c). In addition, measurements were performed for two different dimensions of nanowire width corresponding to $2 \mu \mathrm{m}$ and $1.5 \mu \mathrm{m}$ and the results are shown in Figure 5-9 (d) (i-ii).

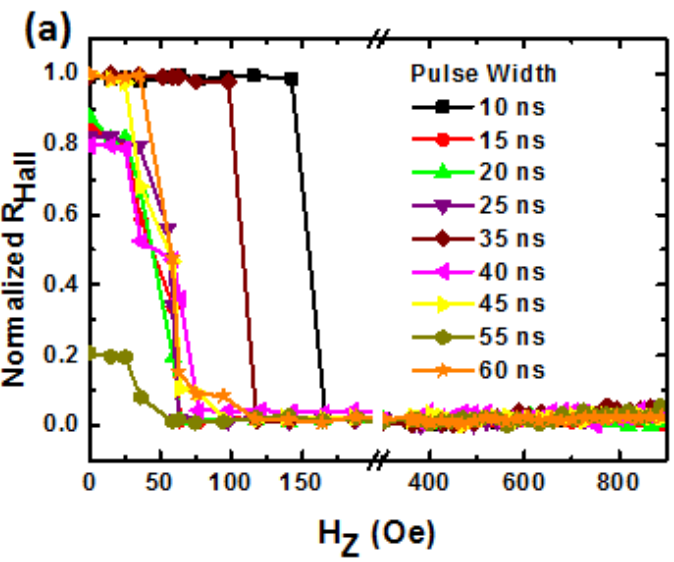

(b)

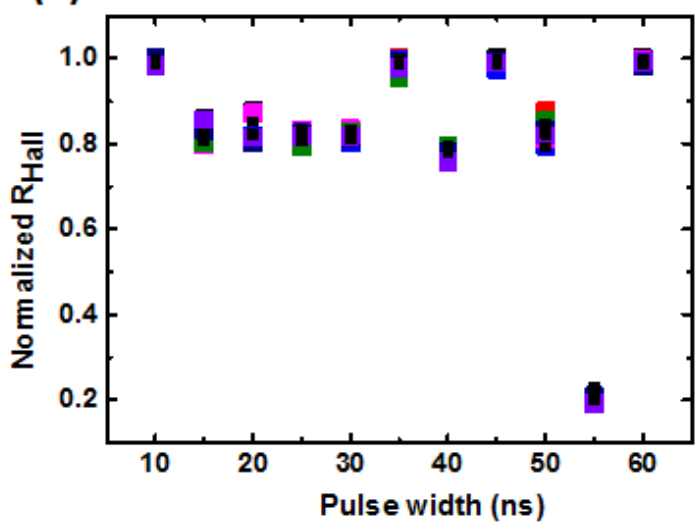

(c)

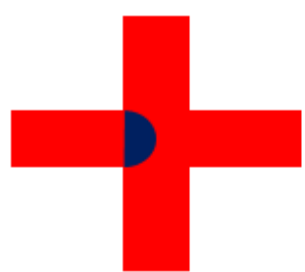

(i)

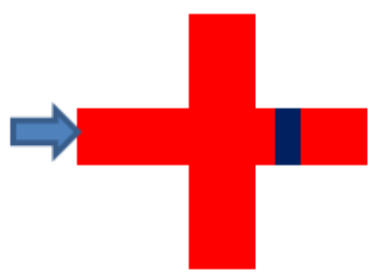

(ii)

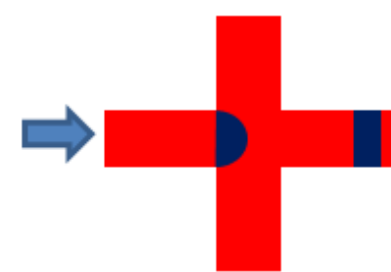

(iii)

\section{Current Flow}

(d)

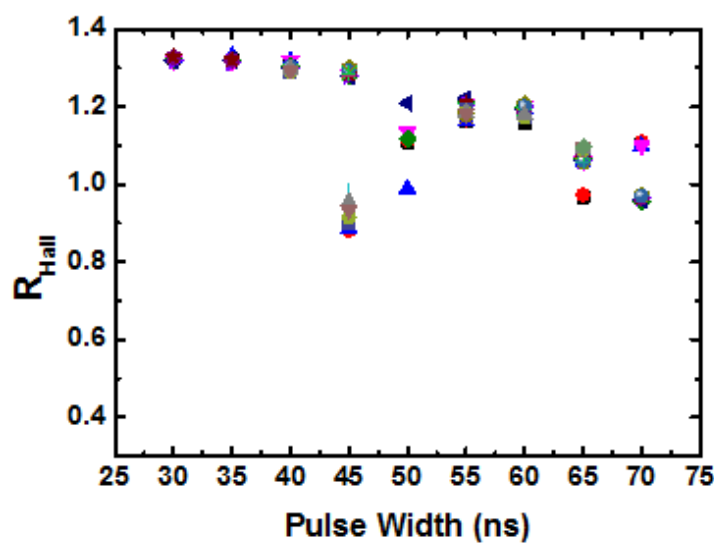

(ii)

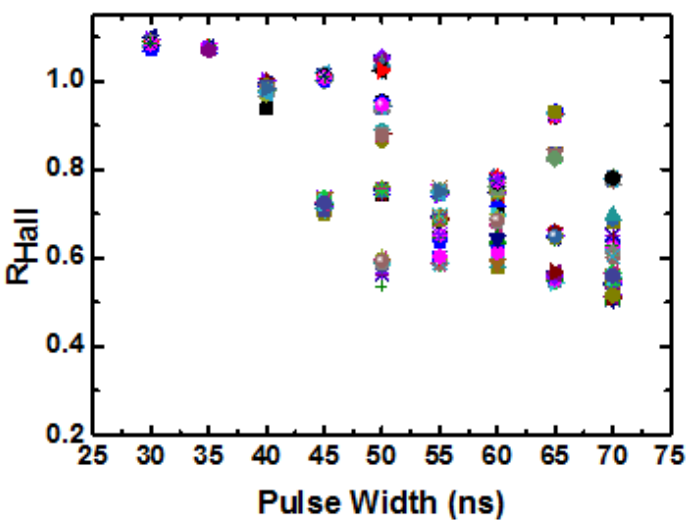

Figure 5-9 AHE measurements on device with Co thickness $=0.4 \mathrm{~nm}$, Ni thickness $=0.8 \mathrm{~nm}$ (sample IX). (a) Normalized $\mathrm{R}_{\text {Hall }}$ variation with external magnetic field as a function of current density. (b) Statistical distribution of $\mathrm{R}_{\text {Hall }}$ as a function of pulse width. Each measurement was repeated 20 times. (c) Schematic depicting a possible scenario showing multiple DW nucleation and propagation across the Hall junction (i) DW nucleation via expansion of reversed magnetic domains at the Hall junction (ii) Depinning and propagation of DW away from the Hall junction (iii) Nucleation and expansion of second DW at the Hall junction. (d) 
Statistical distribution of $\mathrm{R}_{\text {Hall }}$ as function of pulse width for larger nanowire dimensions (i) 2 $\mu \mathrm{m}$ (ii) $1.5 \mu \mathrm{m}$

Although no clear trend is apparent, the measurements are skewed indicating larger drop at larger pulse widths. The magnitude of drop is different for different set of measurements as indicated by the spread in resistance. The large spread indicates variation in the reversed volume near the Hall junction. This indicates the possibility of stochasticity in the DW nucleation process by application of in-plane current.

\subsubsection{In-plane current induced domain wall nucleation process in device with relatively larger anisotropy}

We investigated the effect of pulse current on $\mathrm{R}_{\text {Hall }}$ for devices with larger anisotropy. Sample VII as discussed in section 5.1 with Co thickness $0.4 \mathrm{~nm}$ and Ni thickness $0.4 \mathrm{~nm}$ was chosen. The thin film was patterned into Hall cross structures similar to the one shown in Figure 5-4 (a). The $\mathrm{R}_{\text {Hall }}$ was measured by sweeping the out-of-plane magnetic field, a square hysteresis loop was obtained indicating perpendicular easy axis of magnetization (not shown here). The coercivity of the nanowire obtained was 650 Oe. Figure 5-10 (a) shows the variation of normalized $\mathrm{R}_{\text {Hall }}$ as magnetic field is swept after the application of current pulse in the nanowire. The current density was varied from $6.6 \times 10^{11} \mathrm{~A} / \mathrm{m}^{2}$ to $1.4 \times 10^{12} \mathrm{~A} / \mathrm{m}^{2}$. There was no change in the $\mathrm{R}_{\text {Hall }}$ till the current density was kept below $7.8 \times 10^{11} \mathrm{~A} / \mathrm{m}^{2}$. When the applied current density was $8.9 \times 10^{11} \mathrm{~A} / \mathrm{m}^{2}$, there was a drop in $\mathrm{R}_{\text {Hall }}$ at field orientation but it is not high enough for magnetization reversal at the Hall probe, which occurs at relatively high field strength. As the current density was increased to $1 \times 10^{12} \mathrm{~A} / \mathrm{m}^{2}$, the drop in $\mathrm{R}_{\text {Hall }}$ occurred at much lower field strength (100 Oe). 
(a)

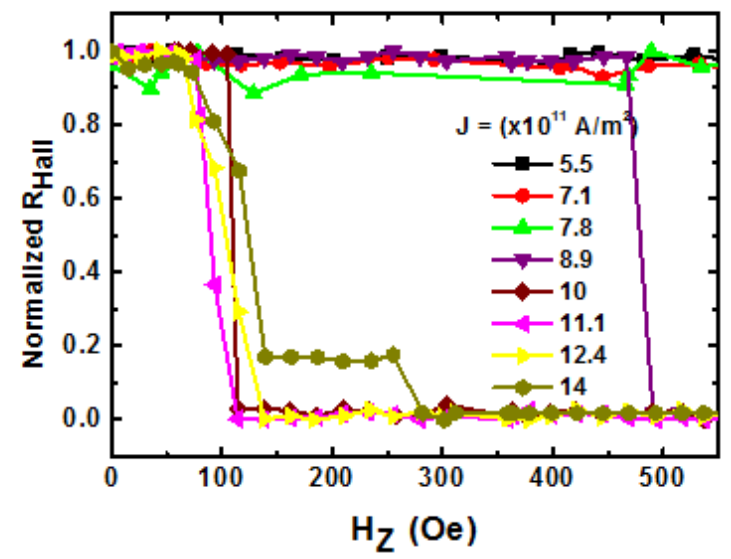

(b)

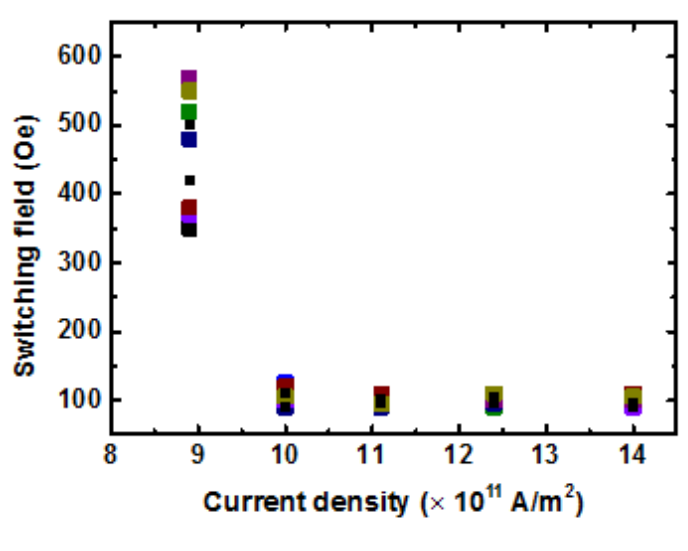

Figure 5-10 AHE measurement on device with Co thickness $=0.4 \mathrm{~nm}$, Ni thickness $=0.4 \mathrm{~nm}$ (sample VII). (a) Normalized $\mathrm{R}_{\text {Hall }}$ variation with external magnetic field as a function of current density. (b) Statistical distribution of switching field with applied current density.

Thus, there is a sudden transition in the magnetization reversal process. The trend in $\mathrm{R}_{\text {Hall }}$ was like the one observed previously as the current density was increased. In all the above results, it is to be noted that there is no change in the magnitude of $\mathrm{R}_{\text {Hall }}$ without field sweep after the pulse injection, unlike in the previous case of lower anisotropy devices, where the $\mathrm{R}_{\text {Hall }}$ was reduced by the application of current itself. This observation points to the fact that magnetization reversal can occur more readily for devices with lower anisotropy strength. Figure 5-10 (b) shows the plot of switching field distribution of the $\mathrm{R}_{\text {Hall }}$ at different applied current density values. Each of these measurements was repeated 20 times to get the distribution of the field at which $\mathrm{R}_{\text {Hall }}$ switches. At current density of $8.9 \times 10^{11} \mathrm{~A} / \mathrm{m}^{2}$, the switching field appeared to have a larger spread. As the current density was increased there was a drop in the switching field and the spread also became narrower. The spread in $\mathrm{R}_{\text {Hall }}$ would depend on the sample geometry and surface irregularities. The nucleation of DWs would be preferably triggered at geometrical in-homogeneities or at the junction of Hall probe and the nanowire. 
(a)

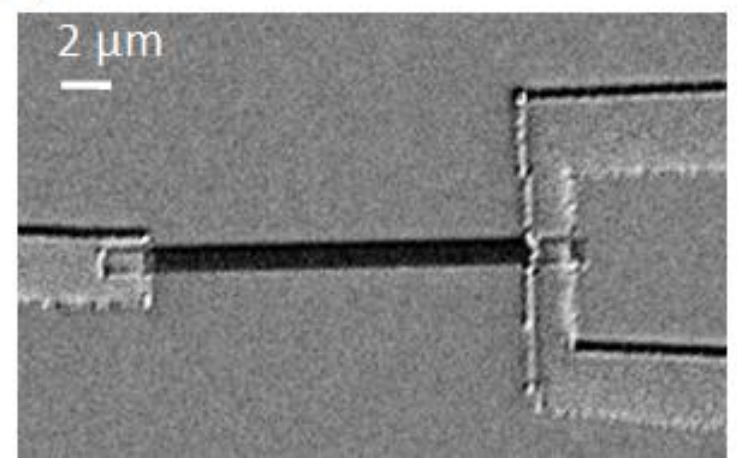

(b)

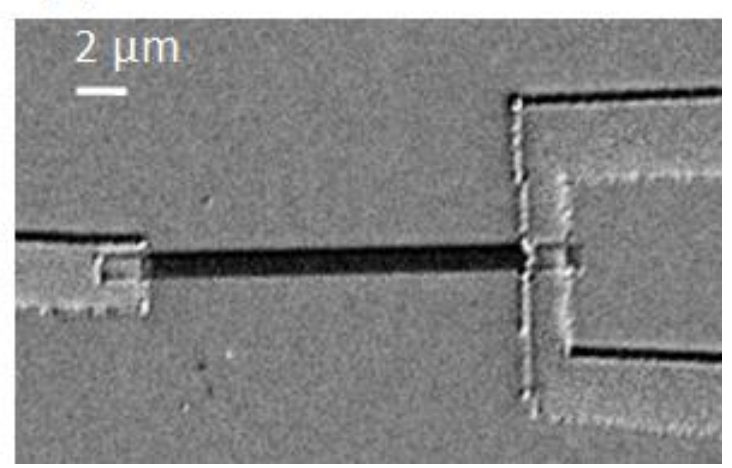

Figure 5-11 (a) Kerr image of nanowire without Hall cross, magnetized along $+z$-direction. (b) Kerr image of nanowire without Hall cross after injection of in-plane current.

\subsubsection{In-plane current injection in nanowire without Hall cross}

We investigated the effect of current pulse injection on nanowire without the Hall cross geometry. Figure 5-11 (a) shows the Kerr image of $1.5 \mu \mathrm{m}$ wide nanowire magnetized along $+z$-direction before the injection of any current pulse. Figure 5-11 (b) shows the Kerr image of the nanowire after injection of current pulses as high as $5 \times 10^{12} \mathrm{~A} / \mathrm{m}^{2}$ and pulse width $100 \mathrm{~ns}$. We did not observe any DW nucleation in this case. This implies that Hall cross plays a crucial role in the nucleation process. Other factors, for instance Joule heating may assist the nucleation process but are not enough to cause the nucleation process on their own.

The thermal activation would be more dominant if $\mathrm{K}_{\mathrm{U}} \mathrm{V} \leq 60 \mathrm{k}_{\mathrm{B}} \mathrm{T}$ [21], where $\mathrm{K}_{\mathrm{U}} \mathrm{V}$ is the product of perpendicular anisotropy constant and the magnetic volume. For our device, $\mathrm{K}_{\mathrm{U}}=$ $1.88 \times 10^{5} \mathrm{~J} / \mathrm{m}^{3}, \mathrm{~V}=1.5 \times 10^{-6}$ (width) $\times 2.8 \times 10^{-9}$ (thickness) $\times 8 \times 10^{-9}(\mathrm{DW}$ width $)$, where DW width is estimated using $\Delta=\sqrt{A / K_{U}}$ and $\mathrm{A}=1 \times 10^{-11} \mathrm{~J} / \mathrm{m}$ [22]. This gives the product $\mathrm{K}_{\mathrm{U}} \mathrm{V}=$ $40 \mathrm{eV}$, which is $1400 \mathrm{k}_{\mathrm{B}} \mathrm{T}$. Thus temperature alone cannot account for compensating the anisotropy. 


\subsection{Magnetic random number generator}

The stochastic nature of DW generation process at the Hall cross and the randomness of the Hall voltage can be utilized to construct a random number generator (RNG) device. Random number generators find applications in online banking, cryptography, simulations and gaming. Since the Hall cross device uses a physical phenomenon as an entropy source, it would function as a true random number generator [23-27], as opposed to a pseudo random number generator which utilizes an algorithm to generate the randomness. Existing CMOS based true RNGs require extensive post-processing to ensure reliable output, which leads to significant power, performance and area overhead [28]. To overcome these issues, magnetic tunnel junction based RNGs have been proposed $[29,30]$, however, they require complex processing steps. The Hall cross based device in this study has simplified processing steps and is compatible with CMOS technology.

Figure 5-12 (a) shows the Hall voltage variation for 200 runs. Each run corresponds to applying a current pulse of density $2 \times 10^{12} \mathrm{~A} / \mathrm{m}^{2}$ and pulse width $50 \mathrm{~ns}$. The random analog output voltage can be converted to a digital signal using voltage amplifier and comparator. The proposed circuit for digital output generation is shown in Figure 5-12 (b). The band-pass amplifier and comparator are packaged in a chip. The Hall sensor would generate an output voltage in the range of $10-50 \mu \mathrm{V}$. Conventional differential amplifiers cannot be used since they suffer limitations from input-offset and noise. To overcome this issue a practical solution is proposed. The sense current for reading the Hall voltage is modulated with the help of a clock, which modulates the output voltage as well. This puts the signal in a higher frequency band compared to the input-offset of the low noise amplifier [31]. The first block in the detection of sense voltage is a differential low-noise amplifier followed by a band-pass filter. The low noise amplifier and filter would amplify the input signal sufficiently so that the 
(a)
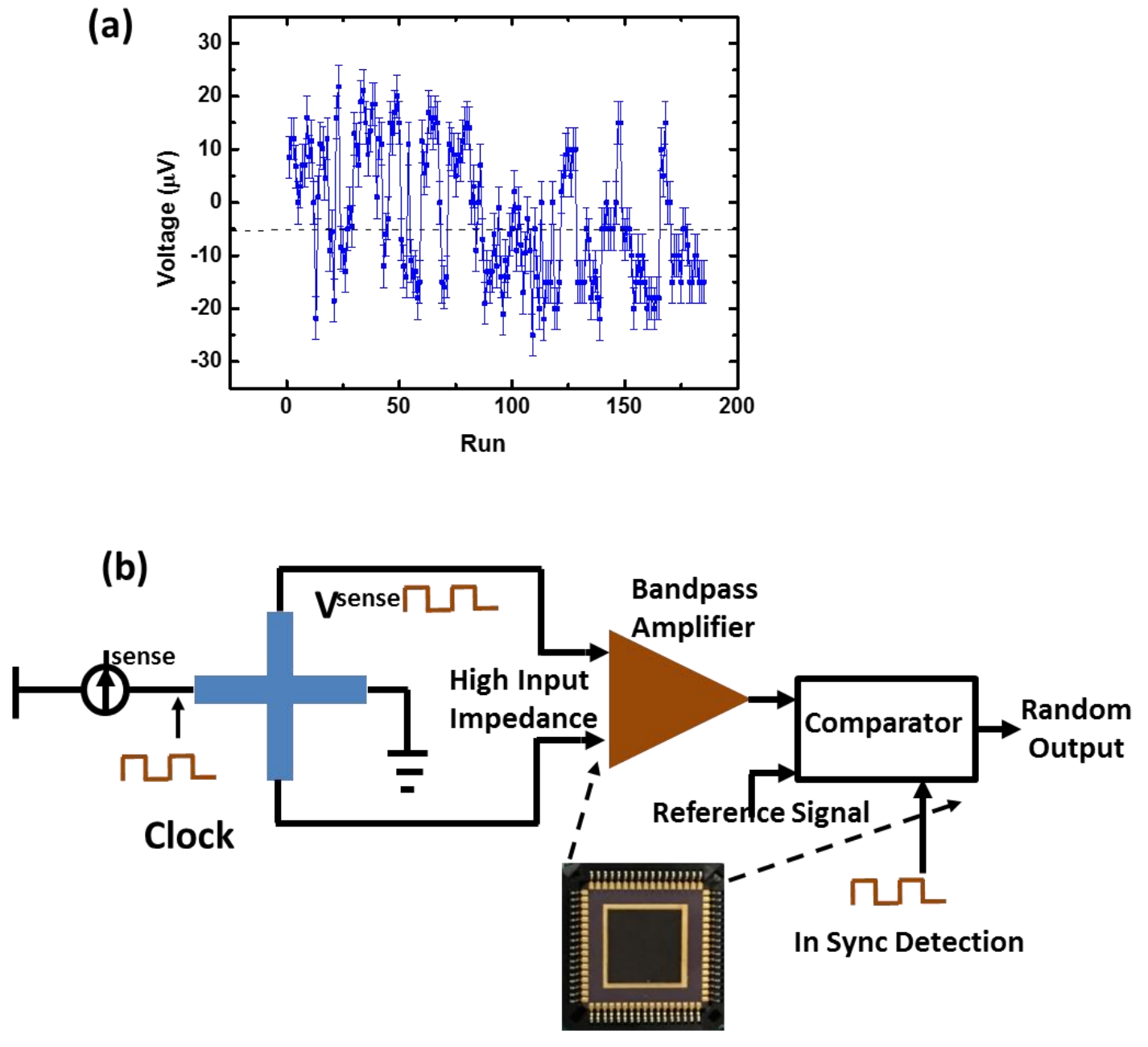

(c)

(i)

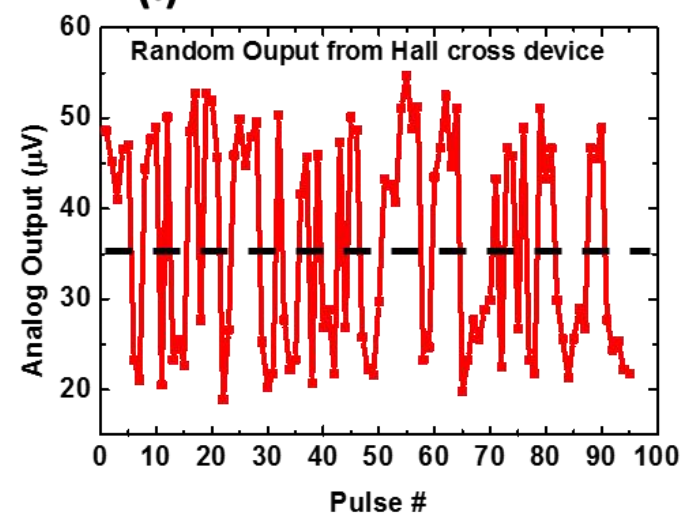

(ii)

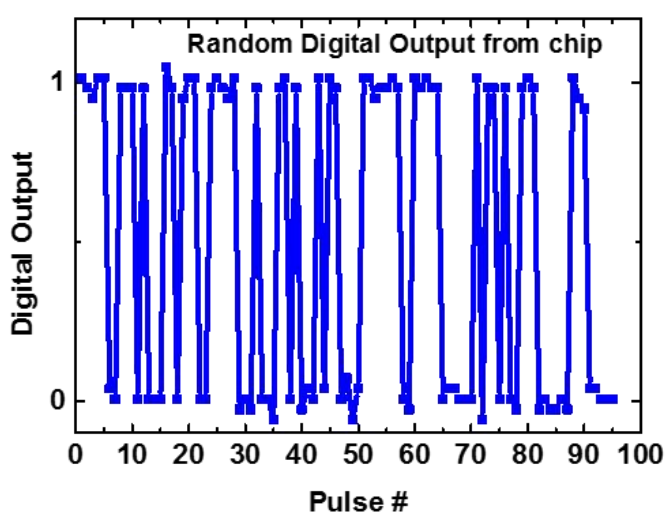

Figure 5-12 (a) Random analog output voltage at the Hall probe across different runs, each run corresponding to $2 \times 10^{12} \mathrm{~A} / \mathrm{m}^{2}, 50 \mathrm{~ns}$ pulse injection. (b) Schematic of proposed read-out circuitry and generation of random digital output. The sense voltage is modulated by a clocked sense current. Schematic of chip is also shown (Circuit in collaboration with Assoc. Prof. Arindam Basu, EEE department, NTU). (c) Test device random output (i) analog voltage at Hall sensor; (ii) digital voltage at chip output. 
effect of the comparator offsets are negligible compared to amplified sense voltage. The bandwidth of the filter is optimized to obtain maximum signal to noise ratio. Figure 5-12 (c-i) shows the analog output obtained from a test Hall cross device. The voltage ranges from 20-50 $\mu \mathrm{V}$. Each pulse would either toggle the output or would remain the same, thus providing a random stream of output. This output is fed to the chip and comparator level is set to a value such that it roughly corresponds to the average of the amplified analog signal. The amplification of the band pass filter is 10000 and average analog voltage is $35 \mu \mathrm{V}$. Thus the comparator level is set to $350 \mathrm{mV}$. In practical devices the comparator level would be set by a micro-controller with an algorithm to detect the average level. Thus when the analog output is above $350 \mathrm{mV}$, the comparator would set the output to digital value 1 and if the analog output is below $350 \mathrm{mV}$, it would set is to digital value 0. The final output is shown in Figure 5-12 (cii).

Figure 5-13 shows a system level schematic integrating the power management unit along with the USB controller. Since the current densities required for output generation are of the order of $10^{12} \mathrm{~A} / \mathrm{m}^{2}$, the power generation circuit can be fabricated by using a special high voltage CMOS process. This circuit would exploit methods to precisely control pulse width and amplitude of programming pulses. The random bits from the Hall probe are streamed to any commercially available serial communication module. Latest peripheral controllers which are equipped with USB 3.0 can support up to $8 \mathrm{GHz}$ throughput. The power management unit would boost the $5 \mathrm{~V}$ available from the host to provide $10-11 \mathrm{~V}$ power to the programming circuit. Thus, following above steps, we can realize a USB plug and play random number generator device. 


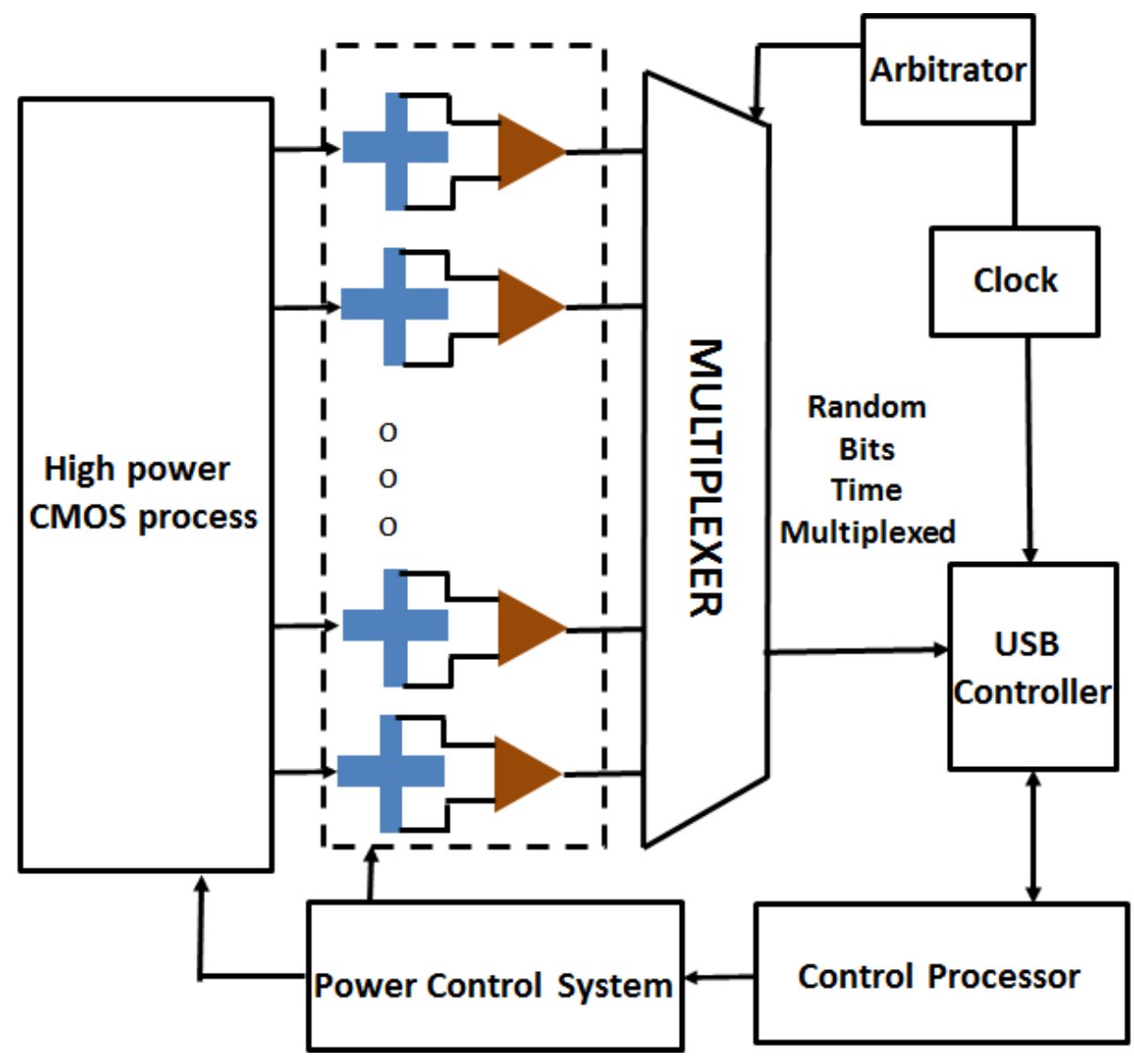

Figure 5-13 System level integration of the random number generator output. Outputs generated from each Hall probe is fed to a peripheral USB controller through a multiplexer. (Assoc. Prof. Arindam Basu, EEE deparment, NTU).

\subsection{Summary}

Domain wall (DW) dynamics are investigated in perpendicular magnetized anisotropy $\mathrm{Co} / \mathrm{Ni}$ nanowires. The magnetic properties of the multilayers were first ascertained at different thicknesses and samples with low anisotropy were selected. Hall cross nanostructures were fabricated and anomalous Hall effect was used to probe the DW dynamics. Field induced DW driving by sweeping an out-of-plane magnetic field and current induced DW driving using spin transfer torque were demonstrated. Without applying local Oersted field to nucleate DWs, application of an in-plane pulse current of current density $\sim 1 \times 10^{12} \mathrm{~A} / \mathrm{m}^{2}$ and pulse width of 50 $\mathrm{ns}$, and then subsequent out-of-plane field sweep causes drop in Hall resistance $\left(\mathrm{R}_{\text {Hall }}\right)$. This is 
attributed to DW nucleation triggered by the anisotropy gradient and higher demagnetization energy at the Hall cross junction. At higher current density, field assistance was not required to nucleate the DWs. Using Kerr imaging multiple DW nucleations were observed near the Hall cross. The spread in $R_{\text {Hall }}$ with the applied current density and pulse width and the presence of multiple DWs indicate the stochastic nature of nucleation process. The device finds a possible application in random number generation with each Hall probe serving as the output signal and in-plane pulse current as the input trigger. 


\section{Bibliography}

[1] A. Yamaguchi, T. Ono, S. Nasu, K. Miyake, K. Mibu, and T. Shinjo, "Real-space observation of current-driven domain wall motion in submicron magnetic wires," Physical Review Letters, vol. 92, Feb 202004.

[2] M. Hayashi, L. Thomas, C. Rettner, R. Moriya, Y. B. Bazaliy, and S. S. P. Parkin, "Current driven domain wall velocities exceeding the spin angular momentum transfer rate in permalloy nanowires," Physical Review Letters, vol. 98, Jan 192007.

[3] D. Ravelosona, D. Lacour, J. A. Katine, B. D. Terris, and C. Chappert, "Nanometer scale observation of high efficiency thermally assisted current-driven domain wall depinning," Physical Review Letters, vol. 95, Sep 92005.

[4] S. W. Jung, W. Kim, T. D. Lee, K. J. Lee, and H. W. Lee, "Current-induced domain wall motion in a nanowire with perpendicular magnetic anisotropy," Applied Physics Letters, vol. 92, May 192008.

[5] G. H. O. Daalderop, P. J. Kelly, and F. J. A. Denbroeder, "Prediction and Confirmation of Perpendicular Magnetic-Anisotropy in Co/Ni Multilayers," Physical Review Letters, vol. 68, pp. 682-685, Feb 31992.

[6] S. Fukami, Y. Nakatani, T. Suzuki, K. Nagahara, N. Ohshima, and N. Ishiwata, "Relation between critical current of domain wall motion and wire dimension in perpendicularly magnetized Co/Ni nanowires," Applied Physics Letters, vol. 95, Dec 72009.

[7] S. H. Yang, K. S. Ryu, and S. Parkin, "Domain-wall velocities of up to $750 \mathrm{~m} \mathrm{~s}(-1)$ driven by exchange-coupling torque in synthetic antiferromagnets," Nature Nanotechnology, vol. 10, pp. 221-6, Mar 2015.

[8] T. Koyama, D. Chiba, K. Ueda, K. Kondou, H. Tanigawa, S. Fukami, et al., "Observation of the intrinsic pinning of a magnetic domain wall in a ferromagnetic nanowire," Nature Materials, vol. 10, pp. 194-197, Mar 2011.

[9] Y. Yoshimura, T. Koyama, D. Chiba, Y. Nakatani, S. Fukami, M. Yamanouchi, et al., "CurrentInduced Domain Wall Motion in Perpendicularly Magnetized Co/Ni Nanowire under In-Plane Magnetic Fields," Applied Physics Express, vol. 5, Jun 2012.

[10] C. Bi and M. Liu, "Reversal-mechanism of perpendicular switching induced by an in-plane current," Journal of Magnetism and Magnetic Materials, vol. 381, pp. 258-262, May 12015.

[11] K. Narayanapillai, X. P. Qiu, J. Rhensius, and H. Yang, "Thermally assisted domain wall nucleation in perpendicular anisotropy trilayer nanowires," Journal of Physics D-Applied Physics, vol. 47, Mar 122014.

[12] L. Q. Liu, O. J. Lee, T. J. Gudmundsen, D. C. Ralph, and R. A. Buhrman, "Current-Induced Switching of Perpendicularly Magnetized Magnetic Layers Using Spin Torque from the Spin Hall Effect," Physical Review Letters, vol. 109, Aug 292012.

[13] T. Phung, A. Pushp, L. Thomas, C. Rettner, S. H. Yang, K. S. Ryu, et al., "Highly Efficient InLine Magnetic Domain Wall Injector," Nano Letters, vol. 15, pp. 835-841, Feb 2015.

[14] M. Yamanouchi, D. Chiba, F. Matsukura, and H. Ohno, "Current-induced domain-wall switching in a ferromagnetic semiconductor structure," Nature, vol. 428, pp. 539-42, Apr 01 2004.

[15] D. Ravelosona, S. Mangin, J. A. Katine, E. E. Fullerton, and B. D. Terris, "Threshold currents to move domain walls in films with perpendicular anisotropy," Applied Physics Letters, vol. 90, Feb 122007.

[16] M. T. Johnson, J. J. Devries, N. W. E. Mcgee, J. A. Destegge, and F. J. A. Denbroeder, "Orientational Dependence of the Interface Magnetic-Anisotropy in Epitaxial Ni/Co/Ni Sandwiches," Physical Review Letters, vol. 69, pp. 3575-3578, Dec 141992.

[17] M. Tang, W. Li, Y. Ren, Z. Zhang, S. Lou, and Q. Y. Jin, "Magnetic damping and perpendicular magnetic anisotropy in Pd-buffered $[\mathrm{Co} / \mathrm{Ni}]_{5}$ and $[\mathrm{Ni} / \mathrm{Co}]_{5}$ multilayers," Rsc Advances, vol. 7, pp. 5315-5321, 2017. 
[18] R. Hiramatsu, K. J. Kim, T. Taniguchi, T. Tono, T. Moriyama, S. Fukami, et al., "Localized precessional mode of domain wall controlled by magnetic field and dc current," Applied Physics Express, vol. 8, Feb 2015.

[19] L. Berger, "Exchange Interaction between Ferromagnetic Domain-Wall and Electric-Current in Very Thin Metallic-Films," Journal of Applied Physics, vol. 55, pp. 1954-1956, 1984.

[20] J. C. Slonczewski, "Current-driven excitation of magnetic multilayers," Journal of Magnetism and Magnetic Materials, vol. 159, pp. L1-L7, Jun 1996.

[21] T. W. McDaniel, "Ultimate limits to thermally assisted magnetic recording," Journal of Physics: Condensed Matter, vol. 17, p. R315, 2005.

[22] S. Fukami, T. Suzuki, N. Ohshima, K. Nagahara, and N. Ishiwata, "Micromagnetic analysis of current driven domain wall motion in nanostrips with perpendicular magnetic anisotropy," Journal of Applied Physics, vol. 103, p. 07E718, 2008.

[23] T. Jennewein, U. Achleitner, G. Weihs, H. Weinfurter, and A. Zeilinger, "A fast and compact quantum random number generator," Review of Scientific Instruments, vol. 71, pp. 1675-1680, Apr 2000.

[24] M. Stipcevic, "Fast nondeterministic random bit generator based on weakly correlated physical events," Review of Scientific Instruments, vol. 75, pp. 4442-4449, Nov 2004.

[25] M. Stipcevic and B. M. Rogina, "Quantum random number generator based on photonic emission in semiconductors," Review of Scientific Instruments, vol. 78, Apr 2007.

[26] M. Furst, H. Weier, S. Nauerth, D. G. Marangon, C. Kurtsiefer, and H. Weinfurter, "High speed optical quantum random number generation," Optics Express, vol. 18, pp. 13029-13037, Jun 7 2010.

[27] S. Robson, B. Leung, and G. Gong, "Truly Random Number Generator Based on a Ring Oscillator Utilizing Last Passage Time," Ieee Transactions on Circuits and Systems Ii-Express Briefs, vol. 61, pp. 937-941, Dec 2014.

[28] S. Yasuda, H. Satake, T. Tanamoto, R. Ohba, K. Uchida, and S. Fujita, "Physical random number generator based on MOS structure after soft breakdown," Ieee Journal of Solid-State Circuits, vol. 39, pp. 1375-1377, Aug 2004.

[29] S. Chun, S. B. Lee, M. Hara, W. Park, and S. J. Kim, "High-Density Physical Random Number Generator Using Spin Signals in Multidomain Ferromagnetic Layer," Advances in Condensed Matter Physics, 2015.

[30] S. Ghosh, "Spintronics and Security: Prospects, Vulnerabilities, Attack Models, and Preventions," Proceedings of the Ieee, vol. 104, pp. 1864-1893, Oct 2016.

[31] M. Tavakoli and R. Sarpeshkar, "An offset-canceling low-noise lock-in architecture for capacitive sensing," Ieee Journal of Solid-State Circuits, vol. 38, pp. 244-253, Feb 2003. 


\section{Chapter 6}

\section{Spin-Orbit Torque Induced Domain-Wall Dynamics in Perpendicular Magnetic Anisotropy Structures}

Efficient magnetization switching is the key to realizing state of the art, high speed and reliable non-volatile memory and logic devices [1-4]. Conventional methods that rely on spin-transfer torque (STT) for magnetization switching $[5,6]$ have limitations as they require materials to spin polarize the current or ferromagnets (FM) with specific spin textures. Recently, a more efficient method of torque transfer has become prominent in systems with FM and heavy metal (HM) interface, namely spin-orbit torque (SOT), for magnetization switching [7-10] and domain wall (DW) driving [11-14]. SOT switching, which does not require materials to spin polarize the current, can arise in systems with strong spin-orbit coupling and inversion asymmetry through the Rashba effect $[7,11]$ and spin Hall effect (SHE) [8, 9, 15]. Although the contribution of Rashba effect and SHE are still under debate, recent studies point towards SHE as the more dominant contributor towards SOT. In SOT, the current flowing through the HM, which is in contact with the FM layer, can switch the magnetization of the FM layer. The commercial application of STT which is magnetic random access memory (STT-MRAM) suffers from tunnel oxide degradation due to high write currents. SOT based MRAM separates the read and write paths since current can be made to flow through just the HM layer and hence increases the device endurance [16]. Current induced effective fields, which are manifestations of the SHE and Rashba effect, are a direct measure of the SOT strength [17-19]. Spin Hall angle (SHA) which is the ratio of spin current to charge current is used to quantify the effect due to SOT. Pt and Ta have been extensively studied as capping and underlayer heavy metals on account of their large SHAs 0.07 and -0.12 respectively [9]. Recent studies have focussed 
on exploring alternate heavy metals possessing larger SHA, for instance, beta phase of W with record SHA of 0.31 [10]. Numerous studies have been carried out to tune the SHA by varying thickness of the heavy metal layer [18-21]. Most of the studies have been confined to single ferromagnetic layer, however at the cost of poor thermal stability which is a major obstacle for scaling down to a few nano-meters.

Néel DWs can be efficiently driven by SOT, even along the current flow direction depending on the sign of SHA and chirality of the DW. An anti-symmetric exchange interaction, termed as Dzyaloshinskii-Moriya interaction (DMI) can stabilize Néel DW of either left-handed or right-handed chirality depending upon the sign of the DMI coefficient [22-24]. Very high DW velocity, e.g. $750 \mathrm{~m} / \mathrm{s}$ was reported in magnetic layers with perpendicular magnetic anisotropy (PMA) coupled with synthetic anti-ferromagnets [25]. DW speed of $400 \mathrm{~m} / \mathrm{s}$ was reported in $\mathrm{Pt} / \mathrm{Co} / \mathrm{AlO}_{\mathrm{x}}$ PMA nanowires on account of Rashba effect [11]. However, the current density required in the above studies was in excess of $3 \times 10^{12} \mathrm{~A} / \mathrm{m}^{2}$. Low current alternative was proposed by Metaxas et al., [26] where vertical current injection was used in a magnetic tunnel junction to drive the DW in the free layer. However, this method requires more complex device processing and the distance propagated by $\mathrm{DW}$ is confined to nanometres.

There is a need of exploring DW dynamics in other multi-layers possessing large SOT strength. One such system that has not been explored extensively for studying DW dynamics is $\mathrm{Pt} / \mathrm{Co} / \mathrm{Ta}$ where the heavy-metal layers provides the SOT and DMI strength. $\mathrm{Pt} / \mathrm{Co} / \mathrm{Ta}$ stack has been reported to exhibit enhanced SOT on account of opposite signs of SHAs of Ta and Pt acting on top and bottom interfaces. However, it suffers from diffusion of Ta into Co which may lead to degradation of PMA [27]. We propose a multilayer stack with four interfaces namely $\mathrm{Pt} / \mathrm{Co} / \mathrm{Pt}$ and $\mathrm{Pt} / \mathrm{Co} / \mathrm{Ta}$, and label it as $\mathrm{Co} / \mathrm{Pt}$ double stack, which has higher thermal stability and stronger PMA than $\mathrm{Pt} / \mathrm{Co} / \mathrm{Ta}$ or $\mathrm{Co} / \mathrm{Pt}$ single stack. We estimated the SHA using 
AC Hall measurements. It was found that increasing the Pt spacer thickness two times reduced the SHA by nearly 1.4 times due to partial cancellation with the underlayer Pt; increasing the Ta capping layer thickness by three times increased the SHA by nearly three times. The current induced magnetization switching increased on increasing the SHA. DW dynamics were investigated in the stack by fabricating Hall cross structures. Hard-axis field was applied to prevent Walker breakdown and also to compensate or support the inherent DMI field. An enhancement in DW velocity was found in the current flow direction on increasing the Ta capping thickness and reducing the Pt spacer thickness.

\subsection{Experimental techniques and comparison of thermal stability for single}

\section{and double Co/Pt stacks}

Thin films of single and double stack $\mathrm{Co} / \mathrm{Pt}$ were deposited on silicon dioxide coated silicon wafers. Sample A, $\mathrm{SiO}_{2} / \mathrm{Ta}(3) / \mathrm{Pt}$ (3)/Co (0.7)/Ta (1) \& sample B, $\mathrm{SiO}_{2} / \mathrm{Ta}$ (3)/Pt (3)/Co $(0.7) / \mathrm{Pt}(0.5) / \mathrm{Co}(0.7) / \mathrm{Ta}(1)$, thicknesses are in $\mathrm{nm}$, were deposited using magnetron sputtering at base pressure of $2 \times 10^{-8}$ Torr. A Pt dusting layer of thickness $1 \mathrm{~nm}$ was deposited on top to prevent oxidation of the capping Ta layer. Since the Pt dusting layer is not in direct contact with the ferromagnetic layer, Co, it would not affect the SOT strength and DW dynamics. The M-H curves were measured for the two as-deposited samples using magneto optical Kerr effect (MOKE) technique. To compare the thermal stabilities of the two stacks, the films were annealed in vacuum at $250^{\circ} \mathrm{C}$ and $350^{\circ} \mathrm{C}$ for 1 -hour and the $\mathrm{M}-\mathrm{H}$ curves were again measured post-annealing. The maximum annealing temperature was chosen as $350^{\circ} \mathrm{C}$ to check for $\mathrm{CMOS}$ process compatibility. Figure 6-1 (a) shows the M-H curves for sample A before and after annealing. The as-deposited sample exhibits square hysteresis with a coercivity of 100 Oe on sweeping the out-of-plane magnetic field. As the sample is annealed to $250^{\circ} \mathrm{C}$, it loses perpendicular anisotropy as indicated by the $\mathrm{M}-\mathrm{H}$ curve, further annealing to $350^{\circ} \mathrm{C}$ causes 
(a)

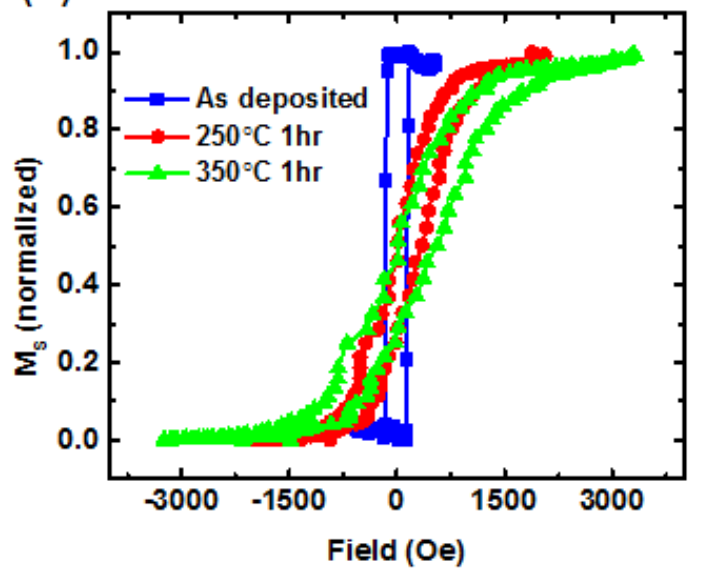

(b)

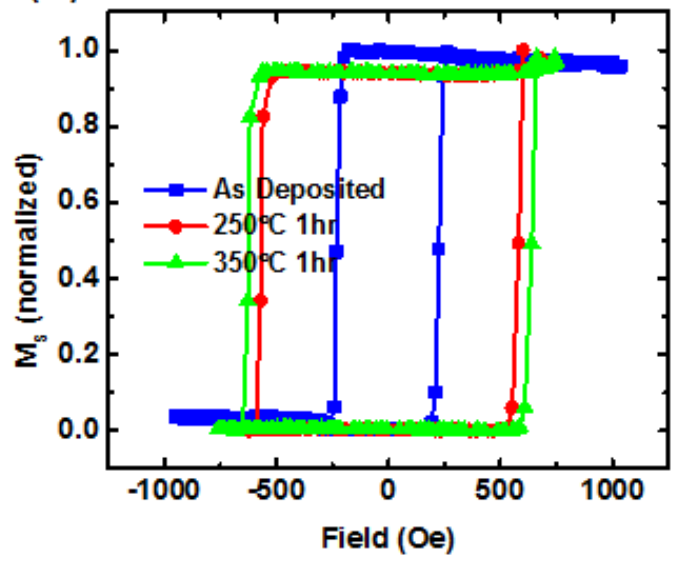

Figure 6-1 (a) M-H loops of thin film stacks, $\mathrm{Pt} / \mathrm{Co} / \mathrm{Ta}$ measured before and after annealing at $250^{\circ} \mathrm{C}$ and $350^{\circ} \mathrm{C}$ for 1 hour. (b) $\mathrm{M}-\mathrm{H}$ loops of thin film stack $\mathrm{Ta} / \mathrm{Pt} / \mathrm{Co} / \mathrm{Pt} / \mathrm{Co} / \mathrm{Ta}$ measured before and after annealing at $250^{\circ} \mathrm{C}$ and $350^{\circ} \mathrm{C}$ for 1 hour.

little change in the $\mathrm{M}-\mathrm{H}$ curve. Figure 1(b) shows the $\mathrm{M}-\mathrm{H}$ curves for sample B before and after annealing. Sample B has a coercivity of 250 Oe before annealing which increases to 500 Oe upon annealing to $250{ }^{\circ} \mathrm{C}$ and the squareness of the hysteresis is maintained, further annealing to $350{ }^{\circ} \mathrm{C}$ leads to very little change in the magnetization. The above results indicate that single stack $\mathrm{Co} / \mathrm{Pt}$ is not thermally stable and has lesser PMA strength than the double stack Co/Pt. The degradation of PMA could be due to inter-diffusion of Ta into Co as was proposed previously by Woo et al. [27] through their x-ray photoelectron spectroscopy (XPS) data. It is worth noting that the stack proposed by Woo et al, consisted of $\mathrm{TaO}_{\mathrm{x}}$ capping to break the inversion symmetry as opposed to our stack. There is lack of evidence to suggest that the presence of $\mathrm{TaO}_{\mathrm{x}}$ would enhance the SOT strength since it is not in direct contact with the ferromagnetic layer, unless the oxygen diffuses to the ferromagnetic layer [28]. The XPS results in fact, indicated a reduction in oxygen intermixing with Co with increase in Ta layer thickness and increase in the Ta layer thickness exhibited enhanced SOT in the stack proposed 
Woo et al. Even though the Co and Ta intermixing would be limited in our stack, we cannot ignore the possibility of Co and Pt intermixing in the stack. However, recent report suggests no significant change in the magnetic quality or PMA degradation due to Co and Pt intermixing [29].We have also performed the XPS measurements for our stack, where we observe no trace of TaOx or Co oxidation and very little intermixing between Ta and the middle Co layer. The results are provided in Appendix A1.

\subsection{Magnetization and spin distribution of Co/Pt double stack device}

We first look at a qualitative picture of the spin current distribution in the heavy-metal layers of Co/Pt double stack on account of SHE. Figure 6-2 (a) shows a schematic depicting spin current distribution in the heavy metal layers of the stack under consideration when an in-plane current flows through it. The distribution takes into account the signs of SHAs of Pt and Ta which are positive and negative, respectively. The spin density is proportional to the heavy metal layer thickness with thicker heavy metals layers carrying more spin current. The bottom ferromagnetic layer, Co, experiences a net Slonczewski-like (SL) torque along the $-y$-direction since the bottom Pt layer is thicker as compared to the spacer Pt layer. The top Co layer would also experience net SL-torque in the $-y$-direction as the contribution from Ta and Pt layer would add up in this case. The combination of above two contributions would give rise to net SL torque along the $-y$-direction. It should be taken into consideration that the double $\mathrm{Co} / \mathrm{Pt}$ stack would have lesser enhancement in SOT as compared to single stack $\mathrm{Co} / \mathrm{Pt}$ due to partial cancellation of the torques from the Pt spacer layer.

In order to test the magnetization anisotropy and current induced SOT switching, Hall cross structures were fabricated using electron beam lithography and Ar-ion milling techniques. Figure 6-2 (b) shows the SEM image of the structures with wire widths of $1.5 \mu \mathrm{m}$. AHE measurements revealed a square hysteresis on sweeping an external out-of-plane magnetic field 
(a)

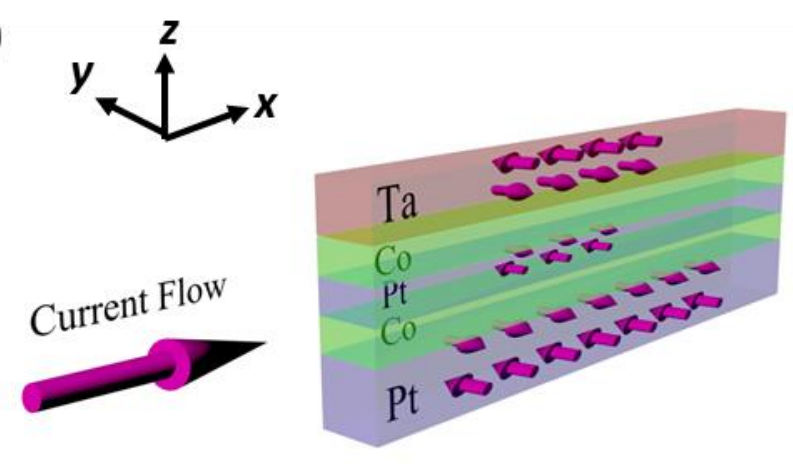

(c)

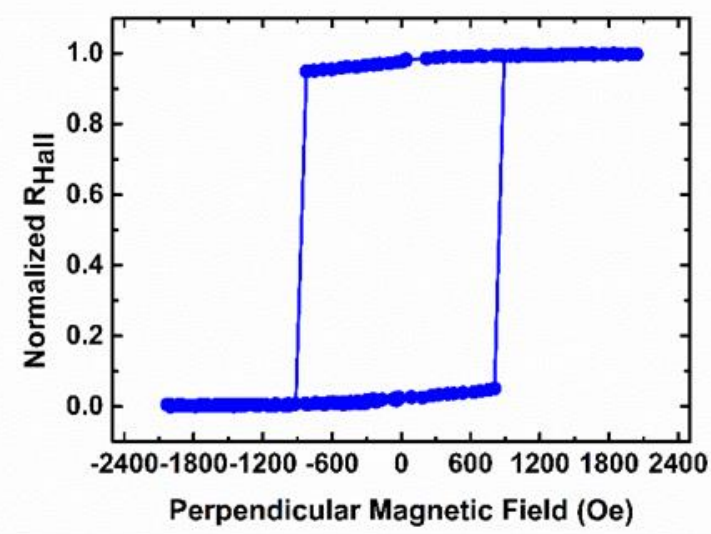

(b)

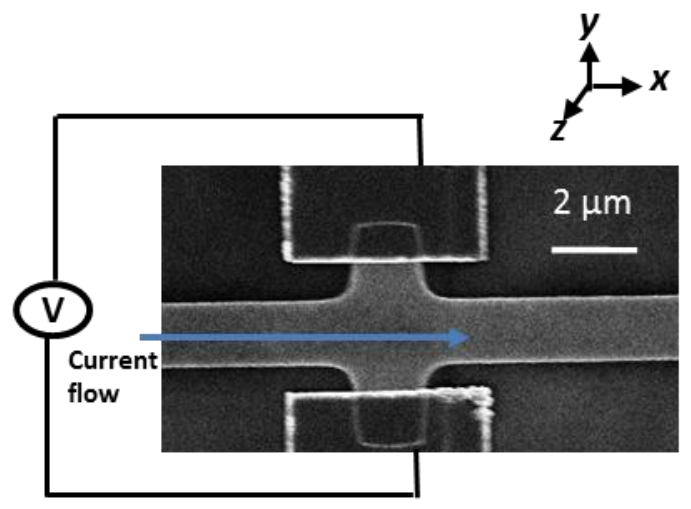

(d)

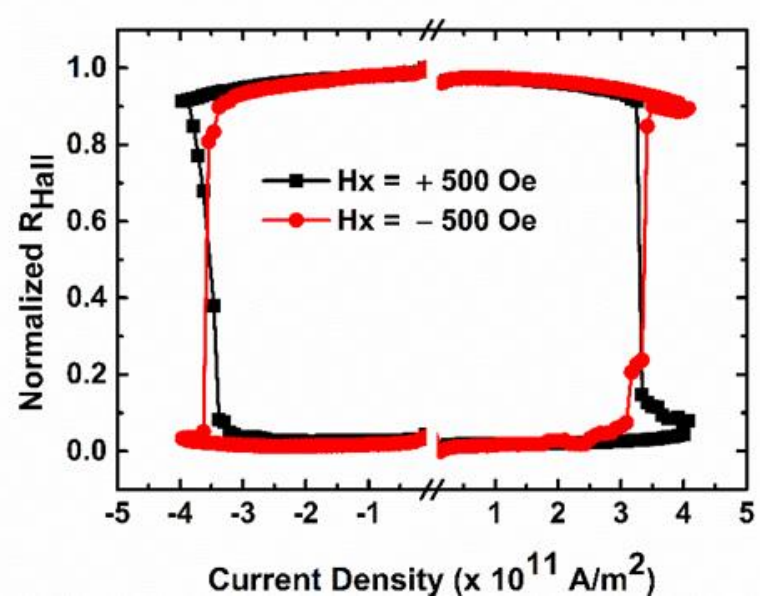

Figure 6-2 (a) Schematic depicting the Co/Pt double stack, in-plane current causes the respective spin current distribution in the heavy-metal layers; the sign of the spin depends on the spin Hall angle and the spin density depends on the thickness. (b) Scanning electron microscopy image of the fabricated Hall cross structure; the schematic depicts set-up for Hall measurements. (c) Dependence of normalized Hall resistance $\left(\mathrm{R}_{\text {Hall }}\right)$ on external out-of-plane magnetic field. (d) Dependence of normalized $\mathrm{R}_{\text {Hall }}$ on sweeping quasi-static in-plane current across the nanowire while applying a longitudinal field of $500 \mathrm{Oe}$.

as shown by the Hall resistance $\left(\mathrm{R}_{\text {Hall }}\right)$ variation in Figure 6-2 (c), indicating the existence of PMA. To validate the existence of SOT in our stack, an in-plane field of 500 Oe was applied and a quasi-static in-plane current was swept across the wire. A hysteretic magnetic switching between up and down magnetization states was observed with the positive in-plane field favouring down magnetization state at positive current, as shown in Figure 6-2 (d). On 
reversing the in-plane field direction, positive current now favoured up magnetization state [30].

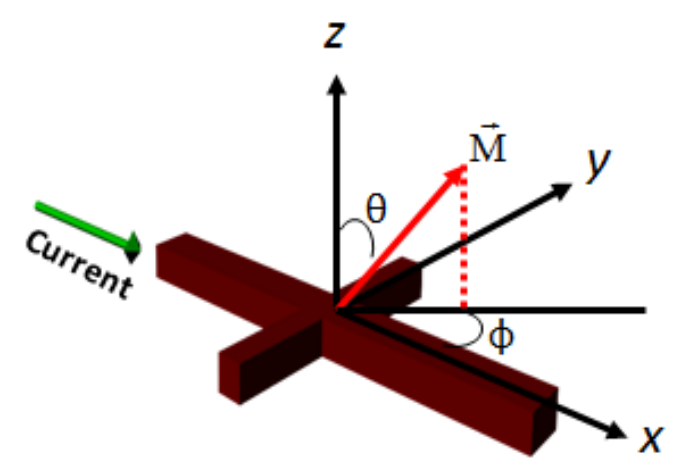

Figure 6-3 Schematic of AC harmonic measurement set-up. Definition of spherical coordinate system is illustrated together with direction of the magnetization, $\mathrm{M}$ and field, $\mathrm{H}$.

\subsection{Harmonic Hall measurements and estimation of spin Hall angle}

The strength of SOT or the SHA can be determined using the technique of harmonic Hall voltage measurements. In this technique, an alternating current is applied across the nanowire and the voltage response is recorded at the Hall bars while sweeping an external magnetic field either in the longitudinal or transverse direction to the nanowire. Using analytical methods, the effective SL field and field-like (FL) fields can be estimated from the voltage response curves. The Hall effect typically has contribution from anomalous Hall effect (AHE) and planar Hall effect (PHE) $[17,31]$. If $\Delta \mathrm{R}_{\mathrm{A}}$ and $\Delta \mathrm{R}_{\mathrm{P}}$ represent changes to the Hall resistance due to AHE and PHE respectively and a current flows in the nanowire along the $\mathrm{x}$-direction as shown in Figure 6-3, then the Hall resistance RXY can be expressed as $[18,31]$

$$
R_{X Y}=\frac{1}{2} \Delta R_{A} \cos \theta+\frac{1}{2} \Delta R_{P} \sin ^{2} \theta \sin 2 \varphi
$$

Application of current induced magnetic fields including the Oersted field can modulate the 
magnetization angle from equilibrium value by an amount $\Delta \theta$ and $\Delta \varphi$. If the magnetization direction in equilibrium has angles $\theta_{0}$ and $\varphi_{0}$ then, the angles in equation (1) can be expressed as $\theta=\theta_{0}+\Delta \theta$ and $\varphi=\varphi_{0}+\Delta \varphi$. The Hall voltage which is the product of Hall resistance and current can be expressed as

$$
V_{X Y}=R_{X Y} I
$$

When a sinusoidal current $(\mathrm{I}=\Delta \mathrm{I} \sin \omega \mathrm{t})$ is applied the current induced effective fields also oscillate in sync with the current. The angles are also modulated as $\Delta \theta \sin \omega t$ and $\Delta \varphi \sin \omega t$. Substituting the modified equation (1) into equation (2) yields the modulated Hall voltage which can also be expressed in terms of the applied signal frequency as [31]

$$
\begin{gathered}
V_{X Y}=V_{0}+V_{\omega} \sin \omega t+V_{2 \omega} \cos 2 \omega t, \\
V_{0}=\frac{1}{2}\left(B_{\theta}+B_{\varphi}\right) \Delta I, \\
V_{\omega}=A \Delta I \\
V_{2 \omega}=-\frac{1}{2}\left(B_{\theta}+B_{\varphi}\right) \Delta I
\end{gathered}
$$

where,

$$
\begin{gathered}
A=\frac{1}{2} \Delta R_{A} \cos \theta_{0}+\frac{1}{2} \Delta R_{P} \sin ^{2} \theta_{0} \sin 2 \varphi_{0}, \\
B_{\theta}=\frac{1}{2}\left(-\Delta R_{A} \sin \theta_{0}+\Delta R_{P} \sin 2 \theta_{0} \sin 2 \varphi_{0}\right) \Delta \theta \\
B_{\varphi}=\Delta R_{P} \sin ^{2} \theta_{0} \cos 2 \varphi_{0} \Delta \varphi .
\end{gathered}
$$

It is shown in reference [31] that the angular term variations $\Delta \theta$ and $\Delta \varphi$ depend on the current induced effective fields, $\Delta \mathrm{H}_{\mathrm{X}}$ along the longitudinal direction and $\Delta \mathrm{H}_{\mathrm{Y}}$ along the transverse direction. Thus second harmonic Hall voltage, $V_{2 \omega}$ contains information about the effective fields through $\Delta \theta$ and $\Delta \varphi$ terms. Following the derivation in reference [31], the respective curvature and slope of $\mathrm{V}_{\omega}$ and $\mathrm{V}_{2 \omega}$ versus the external field are calculated to obtain the ratios 
$\mathrm{B}_{\mathrm{X}}$ and $\mathrm{B}_{\mathrm{Y}}$, defined as $\mathrm{B}_{\mathrm{X}}=\left(\frac{\partial V_{2 \omega}}{\partial H_{X}} / \frac{\partial^{2} V_{\omega}}{\partial H_{X}^{2}}\right)$ and $\mathrm{B}_{\mathrm{Y}}=\left(\frac{\partial V_{2 \omega}}{\partial H_{Y}} / \frac{\partial^{2} V_{\omega}}{\partial H_{Y}^{2}}\right)$. By defining $\xi=\frac{\Delta R_{P}}{\Delta R_{A}}$, finally it can be shown that

$$
\begin{gathered}
\Delta H_{X}=-2 \frac{B_{X} \pm 2 \xi B_{Y}}{1-4 \xi^{2}}, \\
\Delta H_{Y}=-2 \frac{B_{Y} \pm 2 \xi B_{X}}{1-4 \xi^{2}} .
\end{gathered}
$$

The \pm sign corresponds to $\mathrm{M}$ pointing along the $\pm \mathrm{z}$. When PHE is negligible, $\xi=0$, then $\Delta \mathrm{Hx}_{\mathrm{X}}$ and $\Delta \mathrm{H}_{\mathrm{Y}}$ become $2 \mathrm{~B}_{\mathrm{X}}$ and $2 \mathrm{~B}_{\mathrm{Y}}$ respectively.

To estimate SHA, we look at the expression for SOT in terms of the magnetization direction. If $\tau_{1}$ and $\tau_{2}$ represent the SL and FL torque terms respectively, then

$$
\begin{gathered}
\overrightarrow{\tau_{1}}=-\gamma \tau_{1}(\hat{m} \times \hat{\sigma} \times \hat{m}) \\
\overrightarrow{\tau_{2}}=-\gamma \tau_{2}(\hat{m} \times \hat{\sigma})
\end{gathered}
$$

where, $\hat{\sigma}=\hat{\jmath} \times \hat{z}, \hat{\jmath}$ and $\hat{z}$ are unit vectors in the current and out-of-plane directions and $\gamma$ is the gyromagnetic ratio [32]. The spin current can be written as $\Phi_{\mathrm{H}} \mathrm{j}_{\mathrm{c}} / \mathrm{e}$, with $\Phi_{\mathrm{H}}$ representing the SHA. The resulting torque magnitude $\overrightarrow{\tau_{1}}$ can be expressed as [32]

$$
\tau_{1}^{S H E}=\frac{\hbar \Phi_{H} j_{c}}{2 e M_{S} t_{F}}
$$

where, $\mathrm{t}_{\mathrm{F}}$ is the thickness of the ferromagnetic layer, $\mathrm{M}_{\mathrm{S}}$ is the saturation magnetization and $\mathrm{j}_{\mathrm{c}}$ is the conduction electron current. In general, $\tau_{1}^{S H E}>>\tau_{2}^{S H E}$. The SL field $\mathrm{H}_{\mathrm{SLT}}$ and FL field $\mathrm{H}_{\text {FLT }}$ can be determined from equations (5) and (6) as

$$
\begin{gathered}
\overrightarrow{H_{S L T}}=\tau_{1}(\hat{m} \times \hat{\sigma}) \\
\overrightarrow{H_{F L T}}=\tau_{2} \hat{\sigma}
\end{gathered}
$$

Thus, after obtaining $\mathrm{H}_{\mathrm{SLT}}$ from the harmonic measurements as shown in equation (4), SHA can be obtained by substituting in (7) and (8) if other parameters are known. 
The harmonic measurements were performed on sample B after pattering it into a Hall cross.

The frequency was kept as $33 \mathrm{~Hz}$ and in-plane external magnetic field was swept from -600

Oe to +600 Oe. Figure 6-4 (a) shows $\mathrm{V}_{\omega}$ versus $\mathrm{H}_{\mathrm{X}}\left(\mathrm{H}_{\mathrm{L}}\right)$ at a current density of $1.17 \times 10^{11} \mathrm{~A} / \mathrm{m}^{2}$,

(a)

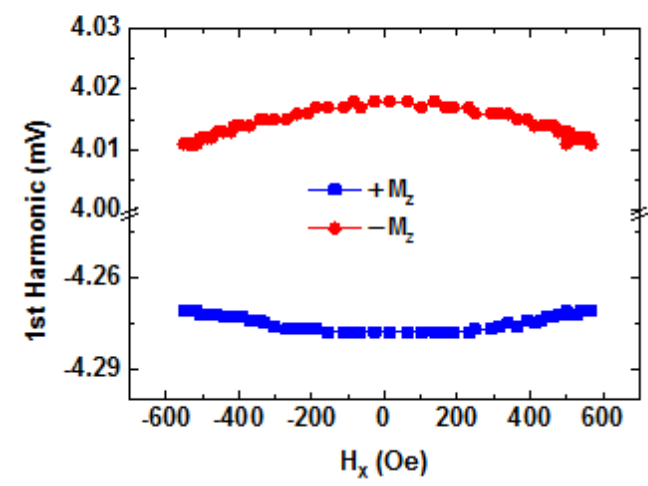

(c)

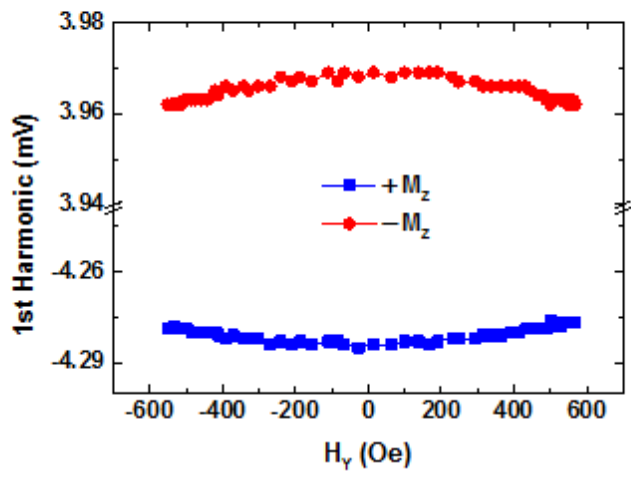

(e)

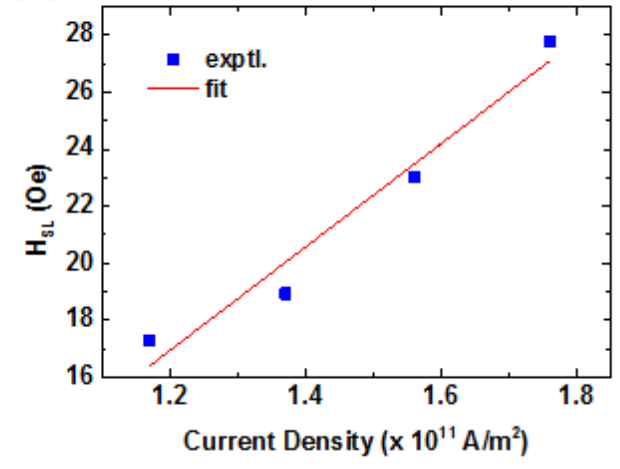

(b)

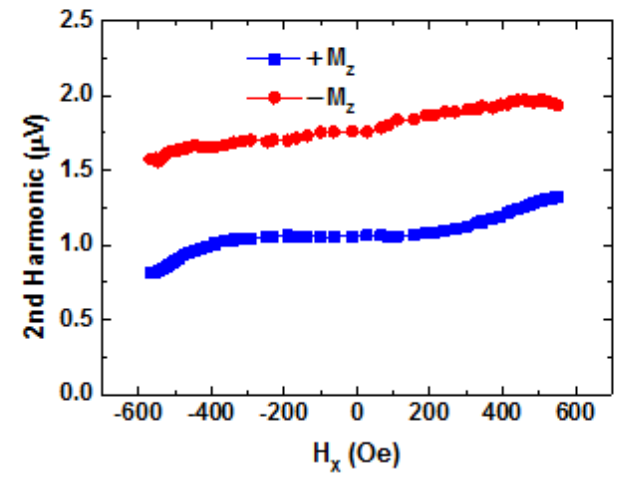

(d)

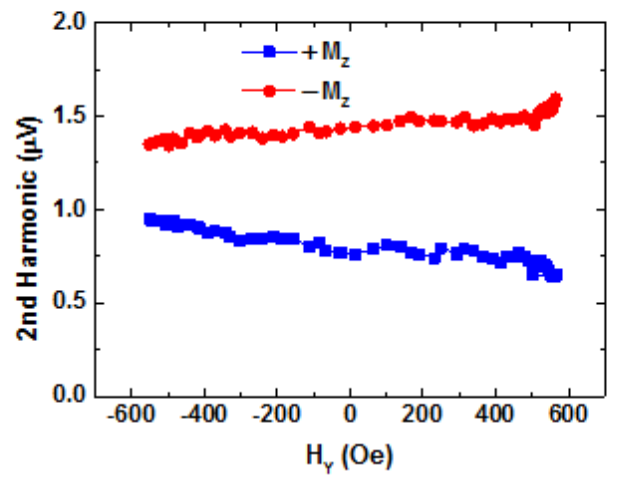

(f)

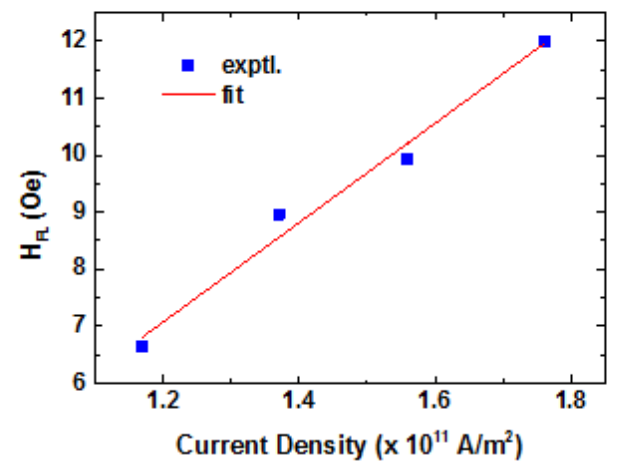

Figure 6-4 Harmonic measurements: Ta capping thickness $=1 \mathrm{~nm}$, Pt spacer thickness $=0.5$ $\mathrm{nm}$ (a) $1^{\text {st }}$ harmonic and (b) $2^{\text {nd }}$ harmonic on sweeping longitudinal field. (c) $1^{\text {st }}$ harmonic and (d) $2^{\text {nd }}$ harmonic on sweeping transverse field. Effective field variation with AC density are shown in (e) for $\mathrm{H}_{\mathrm{SL}}$ and (f) for $\mathrm{H}_{\mathrm{FL}}$. 
measured for up and down magnetized states of the micron-wire. The curves for up and down magnetized states are similar and symmetric with respect to the $x$-axis apart from their opposite signs. A polynomial fitting was performed on the parabolic curve for $+\mathrm{M}_{\mathrm{z}}$ to obtain an equation of the form:

$$
V_{\omega}=B_{1} H_{X}+B_{2} H_{X}^{2}
$$

where $B_{1}$ and $B_{2}$ are the polynomial coefficients of the fitted equation. Figure 6-4 (b) shows $\mathrm{V}_{2 \omega}$ versus $\mathrm{HX}_{\mathrm{X}}$ at a current density of $1.17 \times 10^{11} \mathrm{~A} / \mathrm{m}^{2}$, measured for up and down magnetized states of the micron-wire. The curves for up and down magnetized states have the same slope and are linearly increasing but with different magnitudes. A linear fitting was performed for $+\mathrm{M}_{\mathrm{Z}}$ to obtain an equation of the form:

$$
V_{2 \omega}=B_{0} H_{X}+C
$$

where $B_{0}$ is the slope and $C$ is the intercept of the curve. Substituting from (10) and (11) in (4) gives the following relation

$$
H_{S L}=-\frac{B_{0}}{B_{2}}
$$

A similar relation exists for $\mathrm{H}_{\mathrm{FL}}$, when $\mathrm{V}_{\omega}$ and $\mathrm{V}_{2 \omega}$ are measured versus $\mathrm{H}_{\mathrm{Y}}\left(\mathrm{H}_{\mathrm{T}}\right)$. Figure 6-4 (c) and 6-4 (d) show the respective plots. Variation of $\mathrm{V}_{\omega}$ with $\mathrm{H}_{\mathrm{Y}}$ has an identical trend as that of with respect to $\mathrm{H}_{\mathrm{X}}$. However, $\mathrm{V}_{2 \omega}$ versus $\mathrm{H}_{\mathrm{Y}}$ has opposite signs of slope for $+\mathrm{M}_{\mathrm{Z}}$ and $-\mathrm{M}_{\mathrm{Z}}$ initial magnetizations. The effective fields $\mathrm{H}_{\mathrm{SL}}$ and $\mathrm{H}_{\mathrm{FL}}$, estimated using equation (12) were plotted versus the applied alternating current density and found to vary linearly. Figures 6-4 (e) and 6-4 (f) show the linear relationship of $\mathrm{H}_{\mathrm{SL}}$ and $\mathrm{H}_{\mathrm{FL}}$ respectively, with the current density. $\mathrm{H}_{\mathrm{SL}}$ and $\mathrm{H}_{\mathrm{FL}}$ were evaluated as 18.13 Oe per $10^{11} \mathrm{~A} / \mathrm{m}^{2}$ and 8.7 Oe per $10^{11} \mathrm{~A} / \mathrm{m}^{2}$, respectively, from the slopes of the plots. The SHA $\left(\Phi_{H}\right)$ was estimated for the device by substituting the value of $\mathrm{H}_{\mathrm{SL}}$ in equations (7) and (8). The magnetization, $\mathrm{M}_{\mathrm{S}}$, was measured using alternating gradient force magnetometry (AGFM) and was found to be $800 \mathrm{emu} / \mathrm{cc}$ (shown in Appendix 
A2). We obtained $\Phi_{\mathrm{H}}=0.054$. It should be noted that planar Hall effect correction was negligible in our stack due to the absence of angular dependence of magnetic field on the harmonic voltages.

(a)

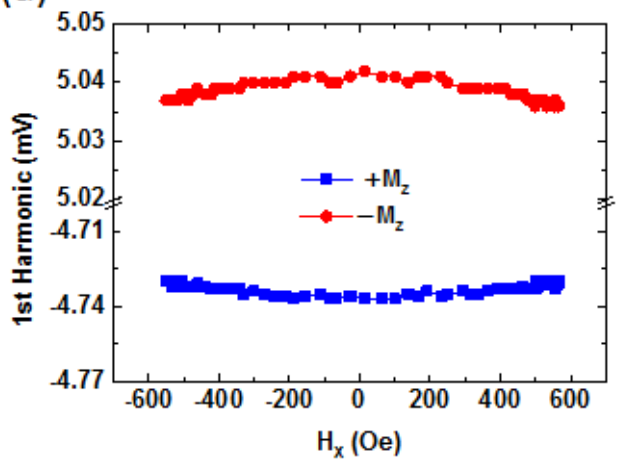

(c)

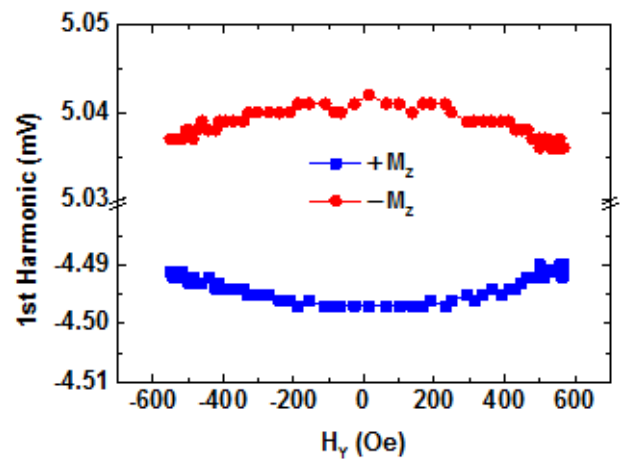

(b)

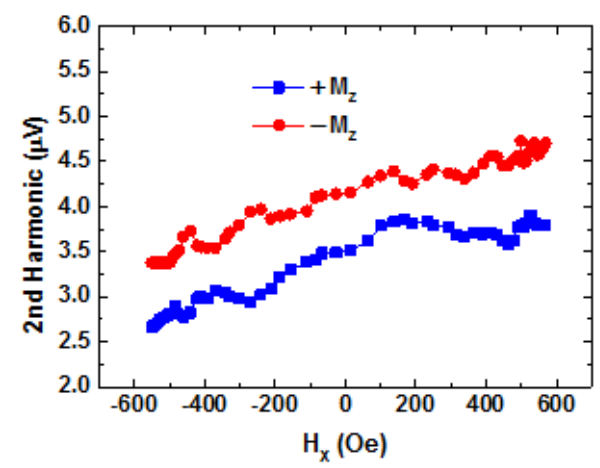

(d)

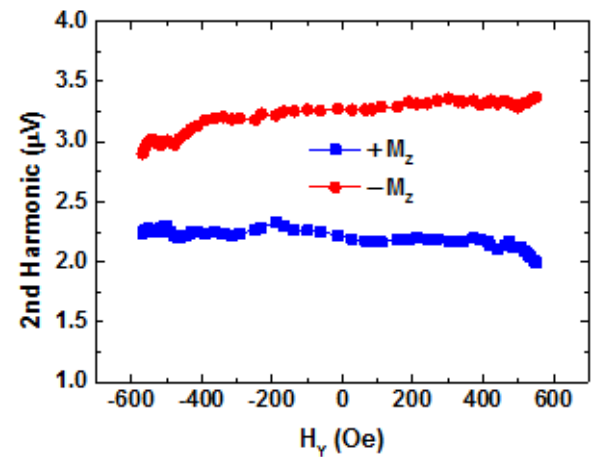

(e)

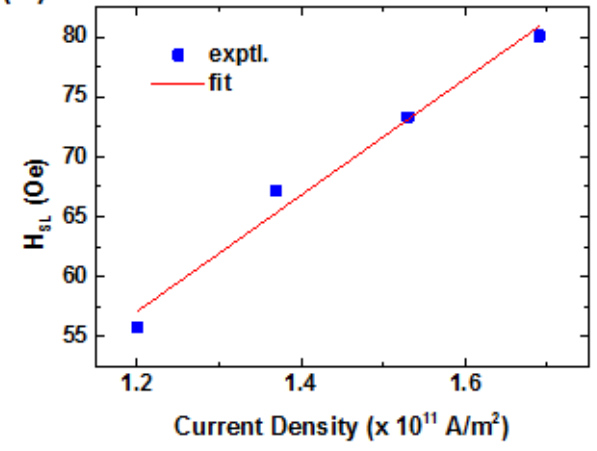

(f)

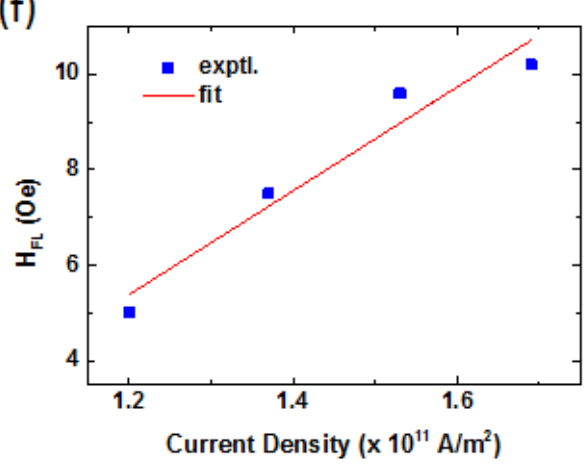

Figure 6-5 Harmonic measurements: Ta capping thickness $=3 \mathrm{~nm}$, Pt spacer thickness $=0.5$ $\mathrm{nm}$ (a) $1^{\text {st }}$ harmonic and (b) $2^{\text {nd }}$ harmonic on sweeping longitudinal field. (c) $1^{\text {st }}$ harmonic and (d) $2^{\text {nd }}$ harmonic on sweeping transverse field. Effective field variation with AC density are shown in (e) for $\mathrm{H}_{\mathrm{SL}}$ and (f) for $\mathrm{H}_{\mathrm{FL}}$. 
To study the effect of Ta capping thickness on the SOT strength, Ta thickness was increased to $3 \mathrm{~nm}$. AC harmonic measurements were performed as before at a current density of $1.53 \times 10^{11} \mathrm{~A} / \mathrm{m}^{2}$. Figures 6-5 (a)-(d) show the plots which have the same trend as Figures 6-4 (a)-(d). Figures 6-5 (e)-(f) show the linear relationship between $\mathrm{H}_{\mathrm{SL}}$ and $\mathrm{H}_{\mathrm{FL}}$ with the applied current density. $\mathrm{H}_{\mathrm{SL}}$ and $\mathrm{H}_{\mathrm{FL}}$ were evaluated as 49.1 Oe per $10^{11} \mathrm{~A} / \mathrm{m}^{2}$ and 10.9 Oe per $10^{11}$ $\mathrm{A} / \mathrm{m}^{2}$, respectively, from the slopes of the plots. The SHA was calculated as 0.15 , considering $\mathrm{M}_{\mathrm{S}}=900 \mathrm{emu} / \mathrm{cc}$ (shown in Appendix A2). Thus on increasing the thickness three times, the spin Hall angle also increased nearly three times. This is due to the enhancement in spin current injection from the heavy metal Ta layer and hence larger torque on account of higher thickness. The thickness of Ta was limited to $3 \mathrm{~nm}$ due to the saturation of spin current density on account of finite spin diffusion length [33, 34].

The effect of Pt spacer thickness was investigated by increasing the thickness to $1 \mathrm{~nm}$ while keeping the Ta capping thickness as $1 \mathrm{~nm}$. AC harmonics measurements were performed as before at a current density of $1.96 \times 10^{11} \mathrm{~A} / \mathrm{m}^{2}$. Figures 6-6 (a)-(d) show the plots, which have the same trend as Figures 6-4 (a)-(d) with the exception that $V_{2 \omega}$ intersect at lower fields for $+\mathrm{M}_{\mathrm{Z}}$ and $-\mathrm{M}_{\mathrm{Z}}$ magnetization directions when transverse field is varied. $\mathrm{H}_{\mathrm{SL}}$ and $\mathrm{H}_{\mathrm{FL}}$ were evaluated as 12.8 Oe per $10^{11} \mathrm{~A} / \mathrm{m}^{2}$ and 5.7 Oe per $10^{11} \mathrm{~A} / \mathrm{m}^{2}$, respectively, from the slopes of the plots. The SHA was calculated as 0.04 , considering $\mathrm{M}_{\mathrm{S}}=820 \mathrm{emu} / \mathrm{cc}$ ( shown in Appendix A2). Increasing the Pt spacer thickness would compensate the SOT from the bottom Pt layer and partially cancel the strength. However, the top ferromagnetic layer would experience some enhancement in the SOT strength. The SHA would depend on the net effect of these torques. 
(a)

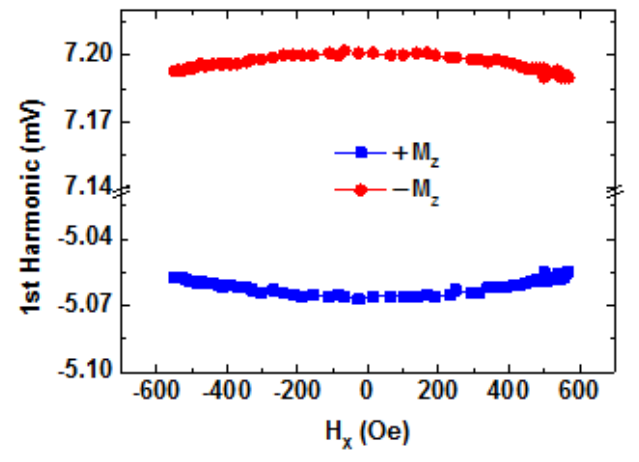

(c)

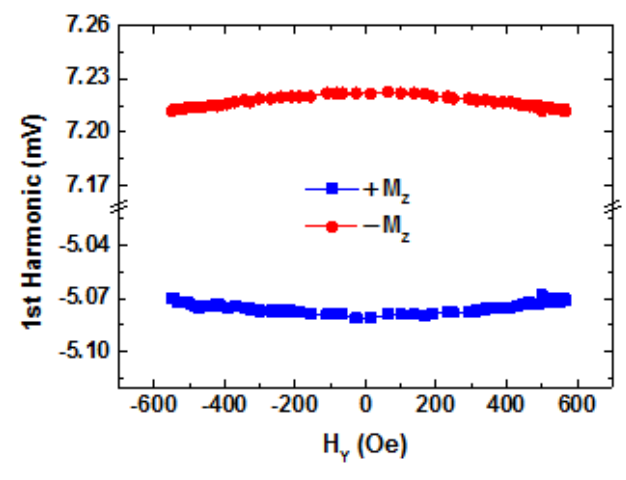

(e)

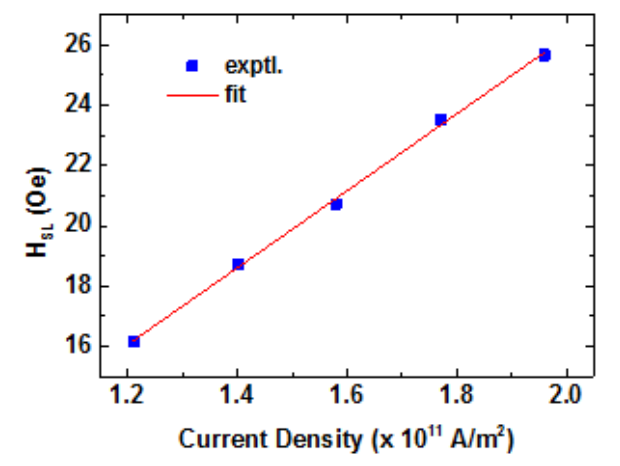

(b)

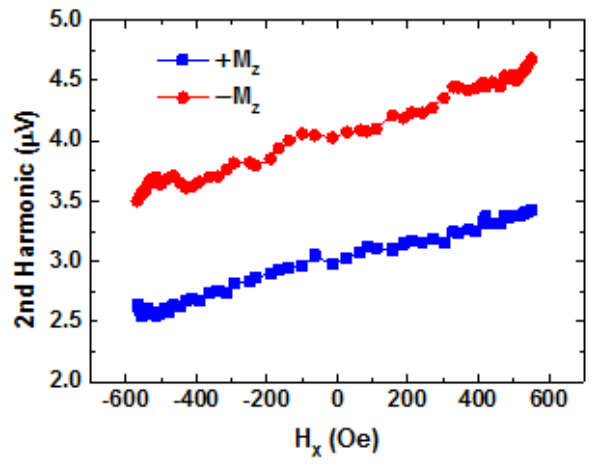

(d)

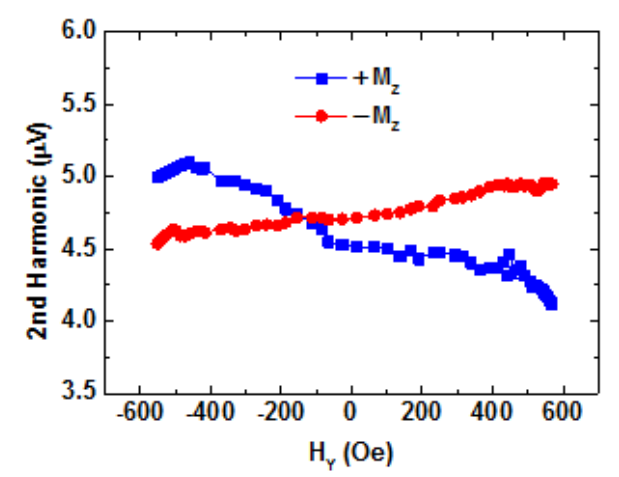

(f)

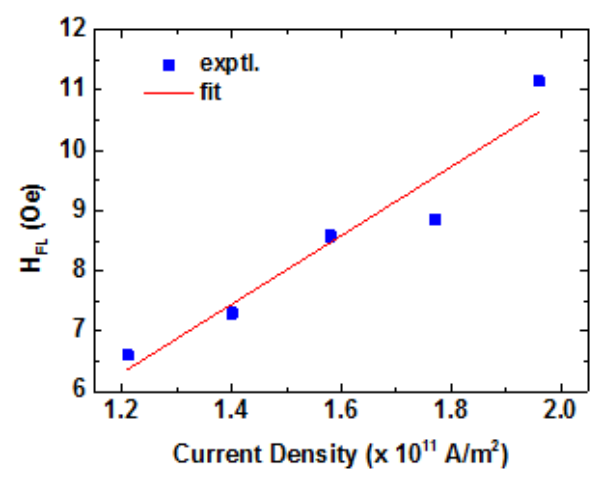

Figure 6-6 Harmonic measurements: Ta capping thickness $=1 \mathrm{~nm}$, Pt spacer thickness $=1 \mathrm{~nm}$ (a) $1^{\text {st }}$ harmonic and (b) $2^{\text {nd }}$ harmonic on sweeping longitudinal field. (c) $1^{\text {st }}$ harmonic and (d) $2^{\text {nd }}$ harmonic on sweeping transverse field. Effective field variation with AC density are shown in (e) for $\mathrm{H}_{\mathrm{SL}}$ and (f) for $\mathrm{H}_{\mathrm{FL}}$. 


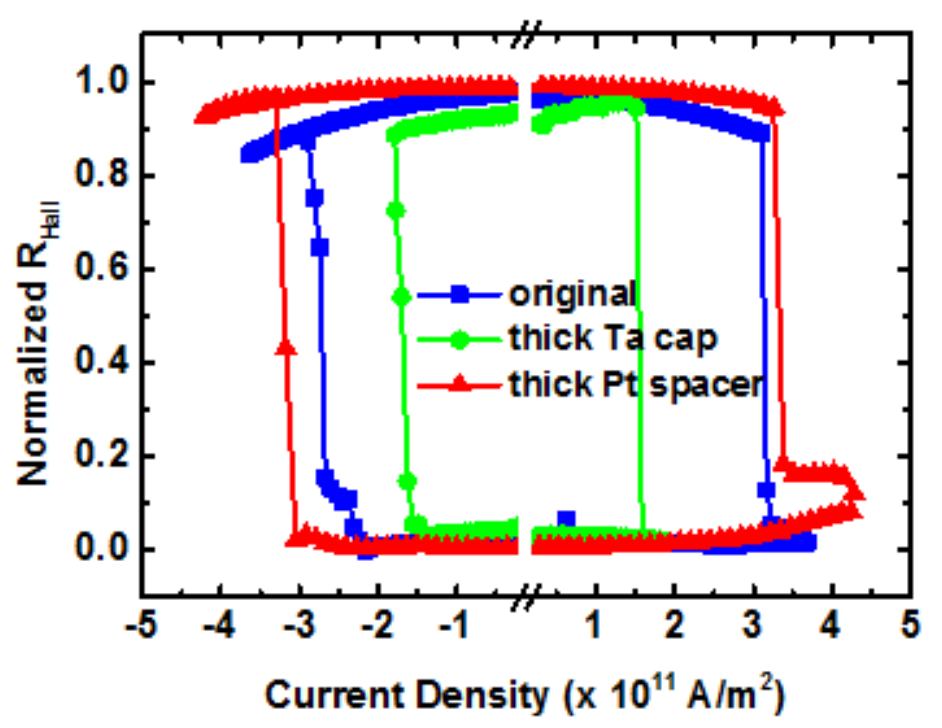

Figure 6-7 Normalized $\mathrm{R}_{\text {Hall }}$ versus in-plane current density depicting magnetization switching comparison between the devices with following stack composition (i) original: $\mathrm{Ta}(3) / \mathrm{Pt}(3) / \mathrm{Co}$ (0.7)/Pt (0.5)/Co (0.7)/Ta (1); (ii) thick Ta cap: Ta (3)/Pt (3)/Co (0.7)/Pt (0.5)/Co (0.7)/Ta (3) and (iii) thick Pt spacer: Ta (3)/Pt (3)/Co (0.7)/Pt (1)/Co (0.7)/Ta (1).

To compute the magnetization switching current density of the devices, an in-plane field of 500 Oe was applied and a quasi-static in-plane current was swept across the wire. A hysteretic magnetic switching between up and down magnetization states was observed with the positive in-plane field favouring down magnetization state at positive current, Figure 6-7 shows the comparison of switching current density for the three devices namely, original: Ta (3)/Pt (3)/Co (0.7)/Pt (0.5)/Co (0.7) Ta (1); thick Ta cap: Ta (3)/Pt (3)/Co (0.7)/Pt (0.5)/Co (0.7) Ta (3) and thick Pt spacer: Ta (3)/Pt (3)/Co (0.7)/Pt (1)/Co (0.7) Ta (1). The current density for switching the original device is $2.8 \times 10^{11} \mathrm{~A} / \mathrm{m}^{2}$, which reduces to $1.5 \times 10^{11} \mathrm{~A} / \mathrm{m}^{2}$ on increasing the Ta capping thickness and rises to $3.1 \times 10^{11} \mathrm{~A} / \mathrm{m}^{2}$ on increasing the Pt spacer thickness. Thus device exhibiting larger effective SOT field and SHA requires lesser current density for switching, making the switching process more efficient. 


\subsection{Spin-orbit torque driven domain wall dynamics in $\mathrm{Co} / \mathrm{Pt}$ multilayers}

DW dynamics were investigated electrically in Co/Pt double stack (sample B) after patterning the thin films into Hall cross structures. The nucleation of DW was performed by injecting pulse current through a stripline patterned orthogonal to the nanowire in a second lithography step. Figure 6-8 (a) shows the injection and driving set-up. A constant in-plane magnetic field was applied along the $-x$-direction to create a left-handed Néel wall. The DW was driven by an application of current pulse and detected by a drop in $\mathrm{R}_{\text {Hall }}$ at the Hall probe due to AHE $[35,36]$. Figure 6-8 (b) shows the SEM image of the fabricated device. The wire width and length were kept as $1.5 \mu \mathrm{m}$ and $30 \mu \mathrm{m}$ respectively. A Ta Hall bar of width $200 \mathrm{~nm}$ was also patterned to detect the position of the DW. The Hall probe was made non-magnetic to prevent DW pinning. Figure 6-8 (b) shows two Hall probes, however in our measurements first Hall probe was used for detection. The distance between the stripline and the first Hall probe was kept as $8 \mu \mathrm{m}$. The DW velocity was determined by noting the pulse width for the DW to reach the Hall probe. This was validated by Kerr microscopy imaging.

Figure 6-8 (c) shows the plot of DW velocity with change in the applied current density under the application of an in-plane field of magnitude 500 Oe. When the field is along the $-x$ direction, the up-down DW propagates along the current flow direction. This is consistent with previous reports which suggest that left-handed Néel wall propagates along the current flow direction, provided the underlayer has positive SHA [12]. The minimum current density for propagation was found to be $3.5 \times 10^{11} \mathrm{~A} / \mathrm{m}^{2}$. The DW velocity increases linearly with the applied current density and is found to reach a value of $440 \mathrm{~m} / \mathrm{s}$ at an applied current density of $1 \times 10^{12} \mathrm{~A} / \mathrm{m}^{2}$. The current density was limited to this value to prevent random domain nucleations and joule heating effect. The DWs were found to propagate along the electron flow direction when the direction of the magnetic field was reversed. Also the minimum current density for finite DW velocity increased to $5 \times 10^{11} \mathrm{~A} / \mathrm{m}^{2}$. The field direction changed 


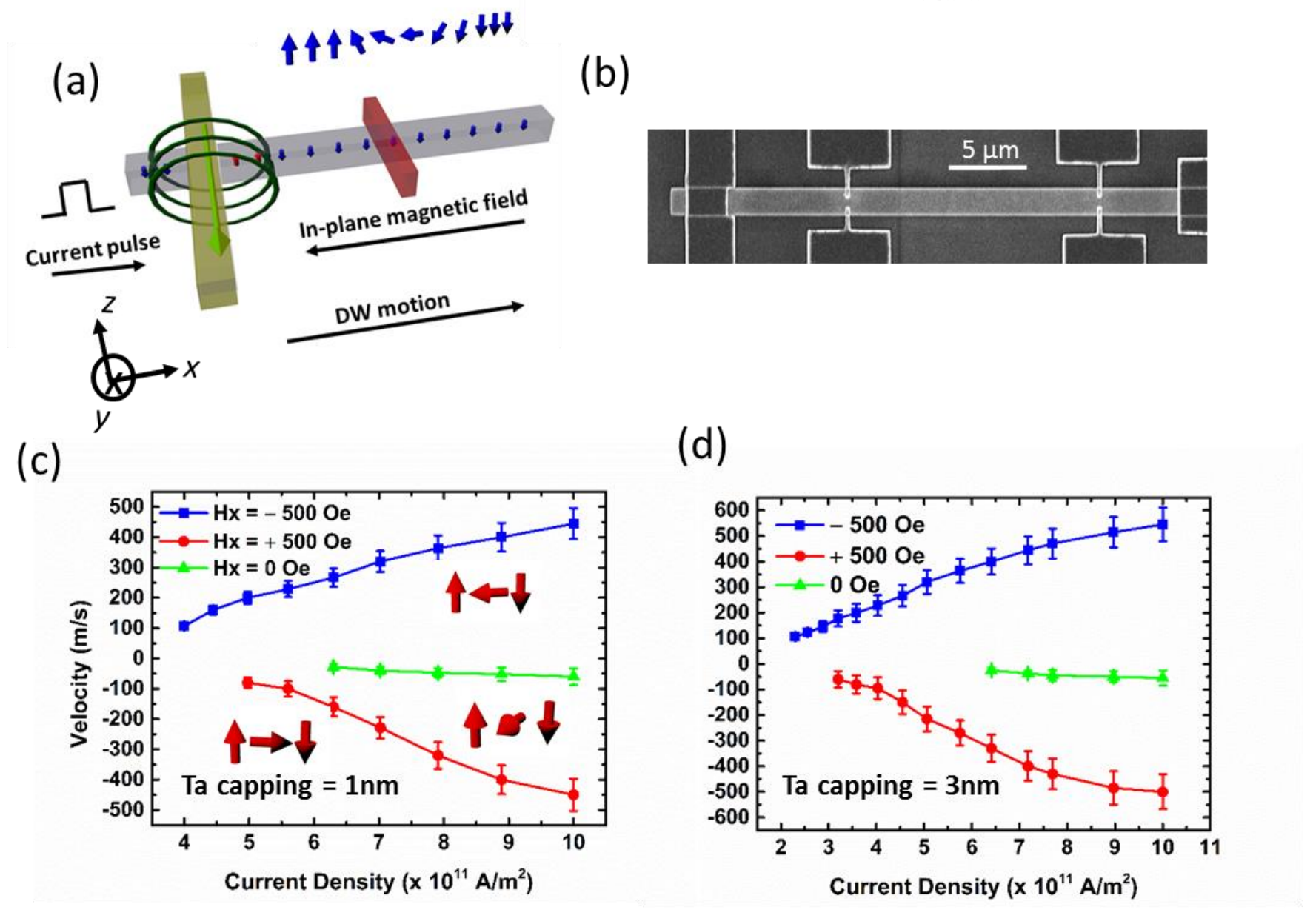

(e)

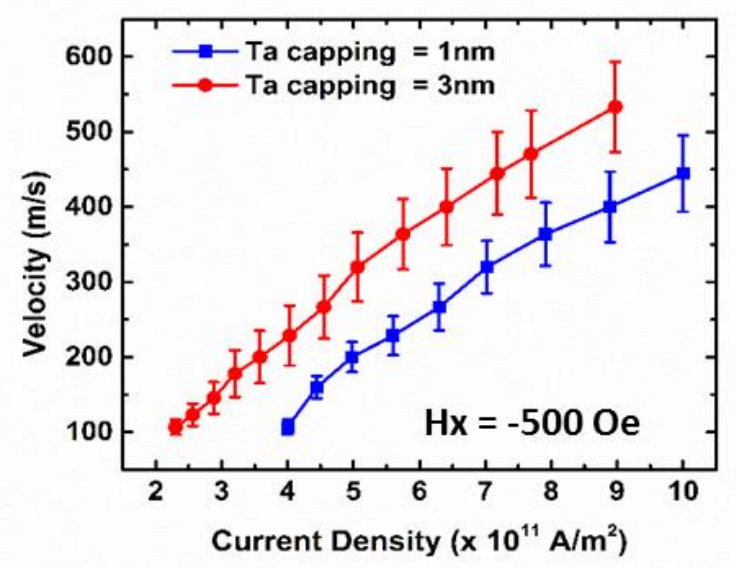

(f)

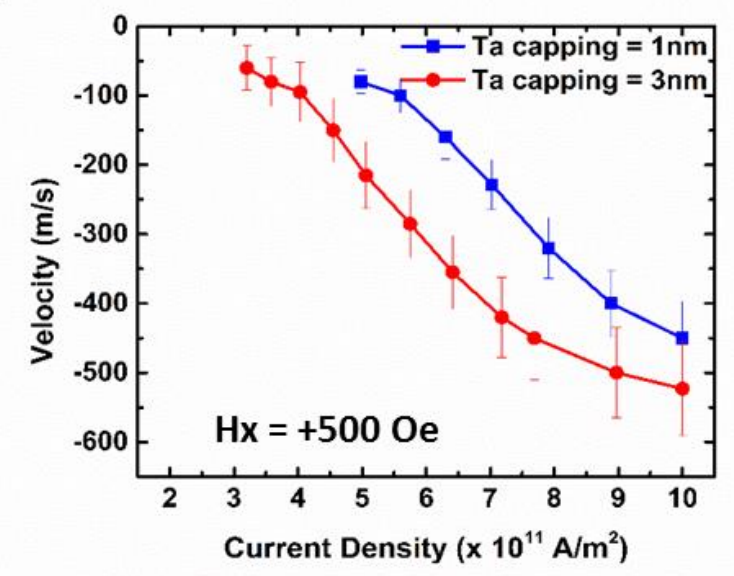

Figure 6-8 (a) Schematic depicting the setup for domain wall (DW) injection and driving measurement. (b) SEM image of the nanowire with non-magnetic Hall probes for DW detection. DW velocity versus current density under a constant in-plane magnetic field along $+x$ and $-x$-directions for Ta capping with thickness (c) $1 \mathrm{~nm}$, and (d) $3 \mathrm{~nm}$. Comparison of DW velocity for Ta capping thicknesses $1 \mathrm{~nm}$ and $3 \mathrm{~nm}$; magnetic field was applied along the (e) $x$-direction, and (f) $+x$-direction. 
the DW chirality to right handed, hence the direction of motion of DW also reversed [12]. At lower current densities, the DW velocity along the current flow direction was observed to be higher than along the electron flow direction. This indicates the existence of a small DMI that favours the left handed Néel chirality and opposes the DMI field. The DMI field, $\mathrm{H}_{\text {DMI }}$ was estimated using DW creep method $[37,38]$ and was found to be 420 Oe. The details are shown in section 6.5. The direction of the DW chirality changed since the applied field is larger than $\mathrm{H}_{\text {DMI. }}$ Against the current flow direction, STT is also expected to contribute to the DW velocity, but its contribution is negligible at lower current densities. The measurement results reveal that the SOT is able to compensate the STT effect when the DW propagates along the current flow direction. When the external field was removed, the DW was found to propagate in the electron flow direction, with minimum current density needed for propagation in excess of $6 \times 10^{11} \mathrm{~A} / \mathrm{m}^{2}$. This indicates the existence of Bloch DW being driven primarily by STT [32]. The magnitude of velocity was found to be much lower than that in the presence of field with maximum velocity around $60 \mathrm{~m} / \mathrm{s}$ at a current density of $1 \times 10^{12}$ $\mathrm{A} / \mathrm{m}^{2}$. We also performed micromagnetic simulations to test the direction of motion of DWs and their structure in the presence of an in-plane field which assists and opposes the motion along the current flow direction. The results are shown in Appendix A3.

\subsubsection{Effect of Ta capping thickness on domain wall velocity}

To investigate the effect of Ta capping thickness, the Ta thickness was increased to $3 \mathrm{~nm}$, keeping the other layer thicknesses fixed including the Pt dusting layer of $1 \mathrm{~nm}$ and the DW velocity was estimated. Figure 6-8 (d) shows the linear relationship of the velocity with the applied current density and has the same trend as that shown in Figure 6-8 (c). Figure 6-8 (e) shows the comparison between DW velocities with $1 \mathrm{~nm}$ and $3 \mathrm{~nm}$ Ta thicknesses respectively, when the applied in-plane field is in the $-x$-direction. The plots indicate that the velocity 
increases nearly 1.5 times when the thickness is raised by 3 times. Increasing the Ta thickness enhances the current flow in the heavy metal layer thereby generating more spin current leading to larger SOT on the ferromagnetic layer. However, the efficiency of the heavy metal to separate the spin components would tend to reduce when the thickness is increased beyond a certain limit on account of finite spin diffusion length. Thus, the thickness of Ta was limited to $3 \mathrm{~nm}$, in our system. Figure 6-8 (f) shows the comparison when the field direction is reversed. In this case also the velocity enhances with increase in the Ta capping thickness. The velocity obtained by increasing the Ta layer thickness is as high as $530 \mathrm{~m} / \mathrm{s}$ which is comparatively higher than reported previously at the applied current density of $1 \times 10^{12} \mathrm{~A} / \mathrm{m}^{2}$. The above electrical measurements were repeated for down-up DW and the results are discussed in Appendix A4.

The electrical measurements were supported by direct imaging of DW motion through magneto-optical Kerr microscopy imaging technique. Figure 6-9 (a) shows the Kerr image of the device with dark contrast indicating magnetization along -z-direction. After DW injection, a current pulse of density $1 \times 10^{12} \mathrm{~A} / \mathrm{m}^{2}$ and pulse width $15 \mathrm{~ns}$ was applied to drive the $\mathrm{DW}$. Kerr image in Figure 6-9 (b) shows that the DW reaches the first Hall probe propagating a distance of $8 \mu \mathrm{m}$ in $15 \mathrm{~ns}$. This gives a velocity of $530 \mathrm{~m} / \mathrm{s}$. Similar measurement was repeated with a pulse width of $18 \mathrm{~ns}$, and the Kerr image in Figure 6-9 (c) shows the DW propagating past the first Hall probe and travelling a distance of $10 \mu \mathrm{m}$. When a $50 \mathrm{~ns}$ pulse was applied the DW moved past the second Hall probe propagating a distance of $25 \mu \mathrm{m}$, as shown in Figure 6-9(d). 
(a)
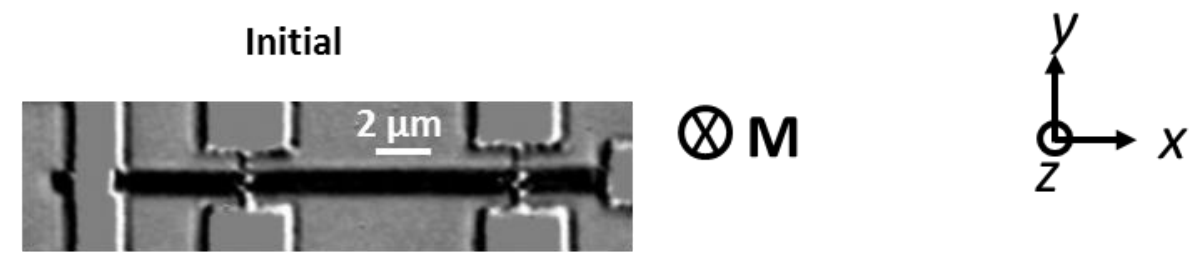

$15 \mathrm{~ns}$

(b)

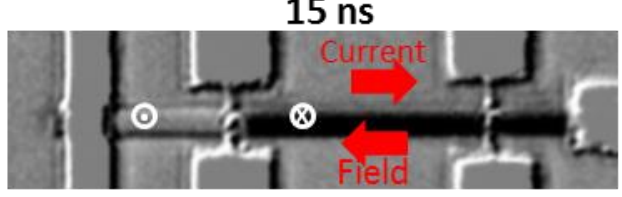

$18 \mathrm{~ns}$

(c)

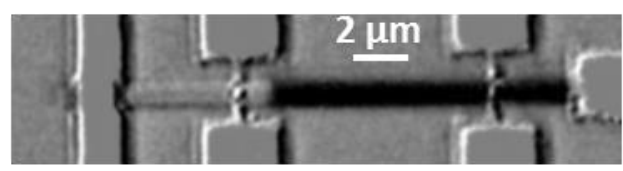

$50 \mathrm{~ns}$

(d)

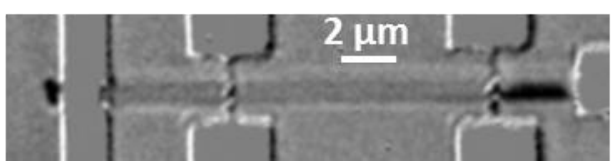

Figure 6-9 Direct observation of SOT-driven DW motion in $\mathrm{Ta} / \mathrm{Pt} / \mathrm{Co} / \mathrm{Pt} / \mathrm{Co} / \mathrm{Ta}$ structure using Kerr imaging technique. (a) Initial magnetization state in $-z$-direction. (b) DW motion after the application of a $15 \mathrm{~ns}$ pulse, the DW propagates $8 \mu \mathrm{m}$ distance and reaches the first Hall probe (c) application of $18 \mathrm{~ns}$ pulse, DW depins from the Hall probe and propagates a distance of 10 $\mu \mathrm{m}$ and (d) application of $50 \mathrm{~ns}$ pulse, DW reaches second Hall probe propagating a distance of $25 \mu \mathrm{m}$.

\subsubsection{Effect of Pt spacer thickness on domain wall velocity}

To examine the effect of Pt spacer thickness on DW velocity, Pt thickness was increased to 1 $\mathrm{nm}$ while keeping Ta capping thickness fixed at $1 \mathrm{~nm}$. Figure 6-10 (a) summarizes the results at different applied current densities. The plots appear symmetric with respect to the $x$-axis. This indicates that the magnitude of the velocity along and against the current flow direction is same when the applied field direction is reversed. Thus this stack has lesser DMI strength than the one with lesser Pt spacer thickness. This comparison is quantified and described in section 
(a)

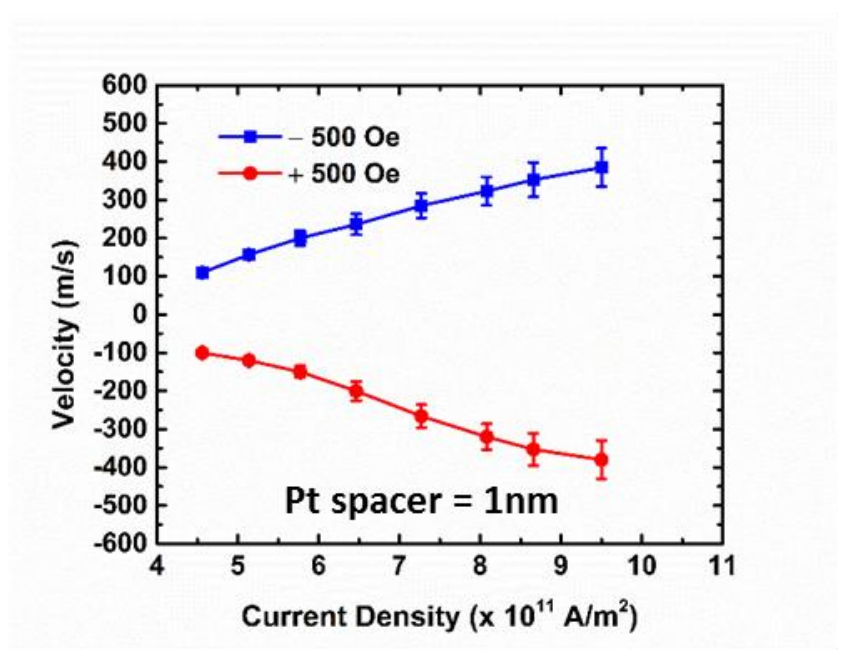

(b)

(c)
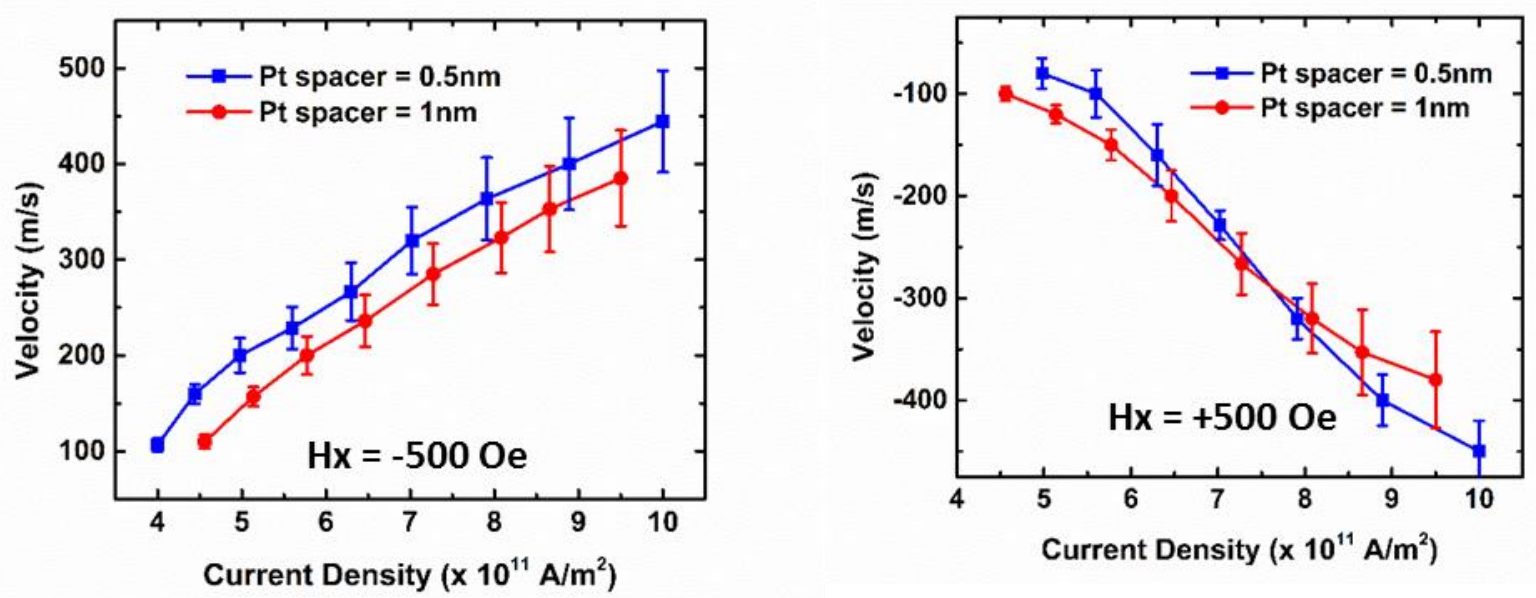

Figure 6-10 (a) DW velocity versus current density in the presence of a constant in-plane magnetic field in $+x$ and $-x$-directions for 1 -nm-thick Pt spacer; plot symmetry across $\mathrm{x}$-axis reveals low DMI strength. DW velocity for Pt spacer with thicknesses $0.5 \mathrm{~nm}$ and $1 \mathrm{~nm}$ when a magnetic field was applied along $(\mathrm{b})-x$-direction, and (c) $+x$-direction. Comparison indicates increasing Pt spacer thickness reduces velocity.

6.5. It also implies that any increment in the velocity by STT is being compensated by the lack of DMI against the current flow direction. This is expected as the DMI from underlayer and spacer Pt would tend to compensate each other if the thicknesses are closer [39]. Figure 6-10 (b) shows the comparison of the velocities between devices with Pt spacer thickness $0.5 \mathrm{~nm}$ and $1 \mathrm{~nm}$ respectively, when the applied field is in the $-x$-direction. Increasing the Pt spacer 
thickness would have two effects: firstly, it would lower the DMI strength; secondly, the SOT would also reduce from the bottom half of the stack, reason being that $\mathrm{Pt} / \mathrm{Co}$ and $\mathrm{Co} / \mathrm{Pt}$ interfaces are competing with each other with regard to the SOT. However, the SOT from the top half of the stack, which is due to the sum of $\mathrm{Pt} / \mathrm{Co}$ and $\mathrm{Co} / \mathrm{Ta}$ interfaces, would increase on increasing the Pt thickness as more spin current would induce torque on the upper Co layer. The net influence of the above contributions would affect the final DW velocity. The reduction in velocity is not very significant, the reason for this is not clear at present, it could be attributed to the net effect of various factors described above.

Figure 6-10 (c) shows the comparison of the velocities when the direction of the field is reversed. When the current density is low, the device with Pt spacer thickness as $1 \mathrm{~nm}$ has larger velocity than the device with $\mathrm{Pt}$ spacer thickness as $0.5 \mathrm{~nm}$. This result is counterintuitive, however, we note that the device with larger Pt spacer thickness has little effect from DMI, hence, when the field is in the $+x$-direction, there is little opposition to the applied field. Whereas, the device with less Pt spacer thickness would experience more resistance to the applied field on account of larger DMI strength. As the current density increases there is a cross-over and beyond current density of $8 \times 10^{11} \mathrm{~A} / \mathrm{m}^{2}$, the DW velocity for the device with Pt spacer thickness $0.5 \mathrm{~nm}$ is larger than the device with spacer thickness $1 \mathrm{~nm}$. This indicates that at larger current density the effect of SOT dominates and it appears that the bottom $\mathrm{Pt} / \mathrm{Co} / \mathrm{Pt}$ stack contribution increases the DW velocity for device with lesser Pt spacer thickness, even though the SOT contribution from the top $\mathrm{Pt} / \mathrm{Co} / \mathrm{Ta}$ stack would be lower. The above electrical measurements were repeated for down-up DW and the results are shown in Appendix A4. 


\subsection{Estimation of Dzyaloshinskii-Moriya interaction strength using domain wall creep method}

Techniques that rely on current induced spin torques to probe the DW characteristics would need to include collective effects of different components (adiabatic, non-adiabatic, spin-Halllike, Rashba-like). The analysis is not straightforward and remains unclear till now. Field induced dynamics, on the other hand, are more simplified to analyse and probe. Field-driven DW in the creep regime have velocities that are governed by a power law, and the dominant term arises from domain wall energy due to DMI. To estimate the field due to DMI, $\mathrm{H}_{\mathrm{DMI}}$, a domain wall is nucleated and then allowed to expand or propagate under a pulsed out-of-plane magnetic field. An in-plane field is applied and the velocity or displacement of the DW is estimated from the Kerr imaging under the combined effect of the magnetic fields. The $\mathrm{H}_{\mathrm{DMI}}$ would oppose the in-plane field if it applied opposite to the chirality or handedness of the DW. HDMI would equal to the in-plane field when the velocity or the displacement is minimum.

Thin film samples of compostion $\mathrm{SiO}_{2} / \mathrm{Ta}(3) / \mathrm{Pt}(3) / \mathrm{Co}(0.7) / \mathrm{Pt}(0.5) / \mathrm{Co}(0.7) / \mathrm{Ta}(1)$, where thicknesses are in $\mathrm{nm}$, were fabricated into $5 \mu \mathrm{m}$ wide micron strips. An injection line was patterned in a second lithography step towards the middle of the wires. The width of the wires was chosen large to get a good contrast and image in the Kerr microscopy measurements. Figure 6-11 shows some of the Kerr images of the device when after injecting two domain walls in the middle, out-of-plane field of pulse width $150 \mathrm{~ms}$ is applied to drive DWs in opposite directions. An in-plane field is also applied along the $-x$-direction to speed up or slow down the DWs. The sequence of Kerr imaging is as follows: first the current is injected in the stripline to nucleate the DWs, which are allowed to expand slightly in the horizontal direction with small out-of-plane field. Then a reference image is taken. After this, the out-of-plane field is applied along with an in-plane field to drive the DWs in opposite directions. The image is again taken and subtracted from the reference. This the reason that in the images 
(a)
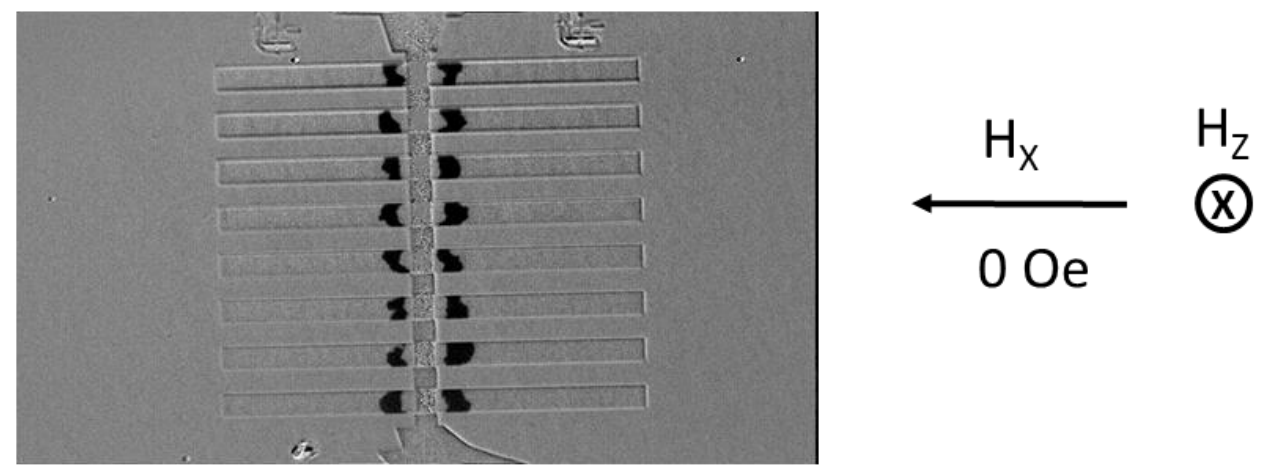

(b)
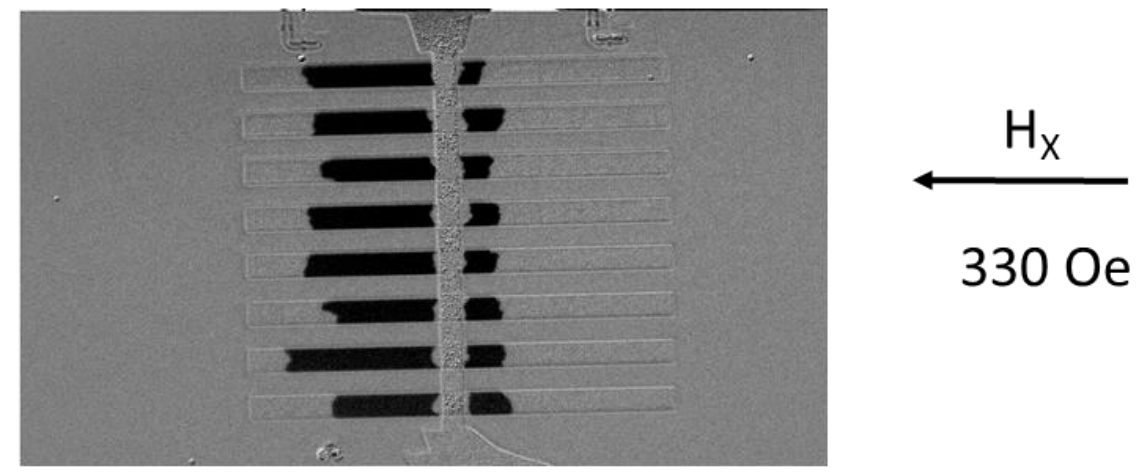

(c)

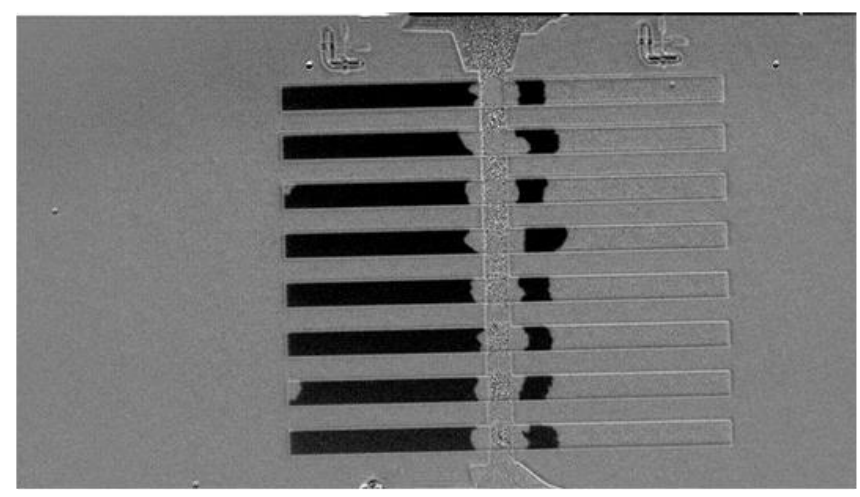

(d)

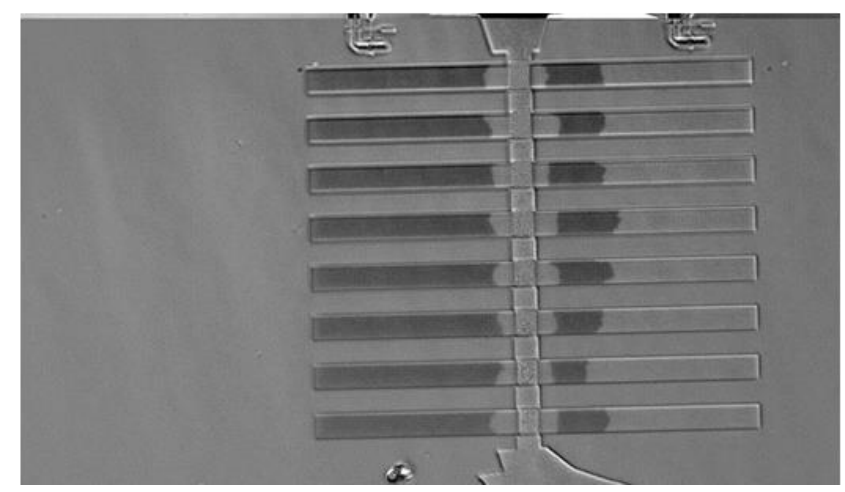

Minimum Right Side Domain Expansion

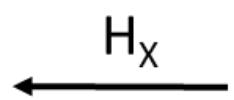

440 Oe

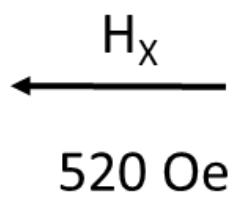

Figure 6-11 Kerr imaging to show DW creep in the presence of out-of-plane and in-plane magnetic field along - $x$-directionequal to (a) 0 Oe; (b) 330 Oe; (c) 400 Oe; (d) 520 Oe. The inplane field opposes DMI field for DW propagating towards right and minimum displacement occurs at field equal to DMI field. Here $\mathrm{H}_{\mathrm{DMI}}=420-440$ Oe. 


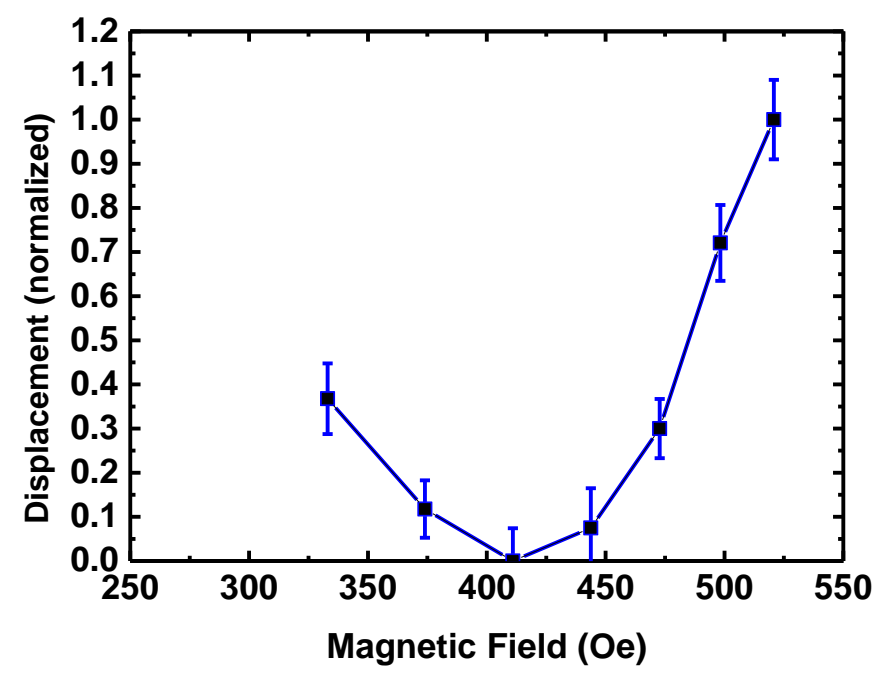

Figure 6-12 Domain wall displacement as a function of in-plane magnetic field. Minimum displacement corresponds to DMI field $=420$ Oe.

shown below, the region next to the stripline appears without any domain (or gray in color as the background) since it is subtracted. It is observed that the domains are not perfect since the widths of the nanowires are large and there is a possibility of multiple domans or pinning sites deforming the domains. The images shown in Figure 6-11 are for gradually increasing in-plane field in the $-x$-direction. The field values are indicated in the images. The dark contrast represents down magnetization. Since the DWs have left hand Neel chirality, the DWs on the left of the stripline would always propagate faster since the in-plane field is assisting it. The DWs on the right of the stripline would propagate slower since the in-plane field would oppose field due to DMI or $\mathrm{H}_{\mathrm{DMI}}$. For applied in-plane field much less than $\mathrm{H}_{\mathrm{DMI}}$, the DWs propagate at reasonable speed. As the field is increased towards the $\mathrm{H}_{\mathrm{DMI}}$, the speed or the displacement as observed in the Kerr images start to drop. The speed was found to be minimum at around 400 Oe of applied in-plane field. When the applied field increases beyond $\mathrm{H}_{\mathrm{DMI}}$, it overcomes the DMI field and the DW is able to expand again at a faster rate. 
(a)
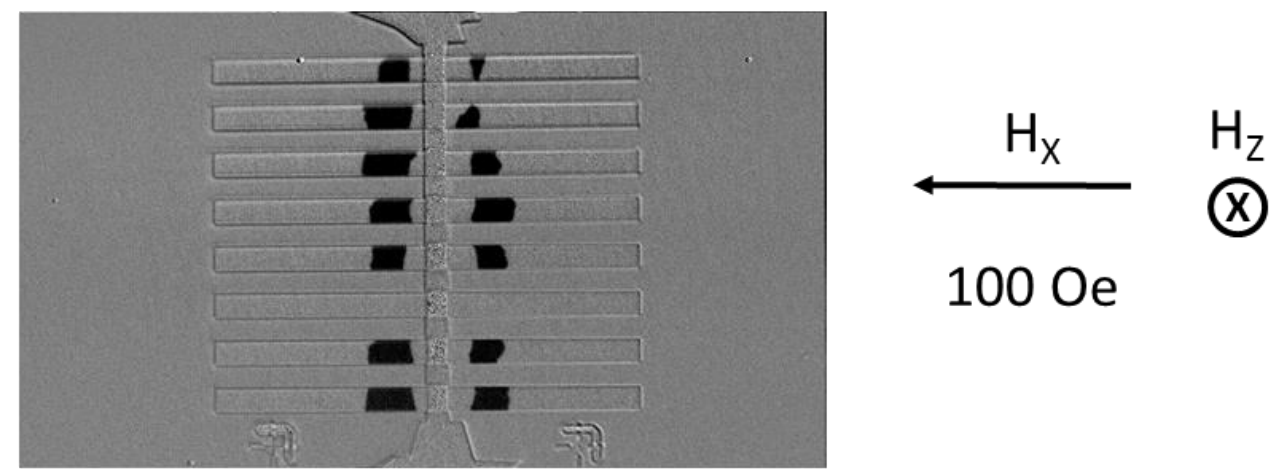

(b)

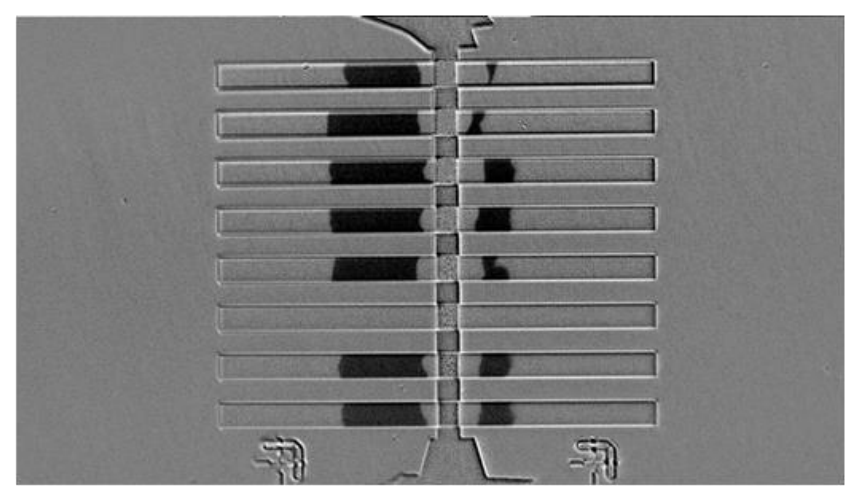

Minimum Right Side Domain Expansion $\stackrel{\mathrm{H}_{\mathrm{X}}}{\longleftarrow}$ $185 \mathrm{Oe}$

(c)
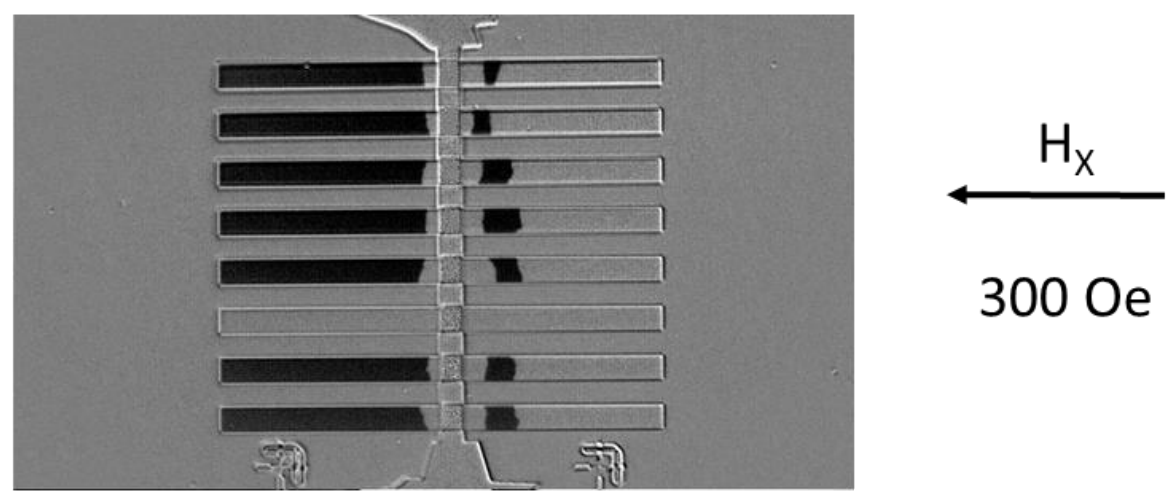

(d)
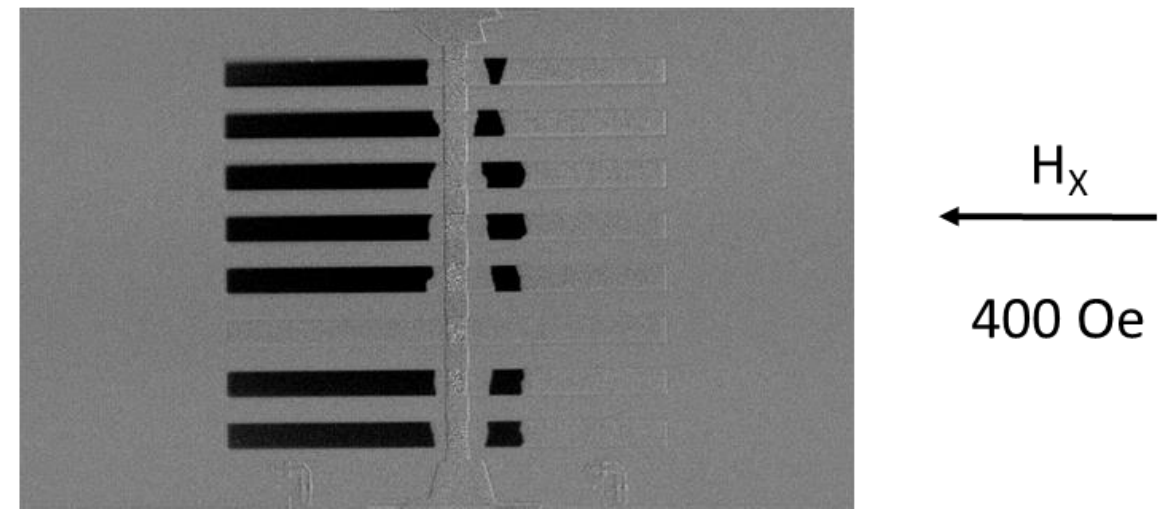

Figure 6-13 Kerr imaging to show DW creep in the presence of out-of-plane and in-plane magnetic field along $-x$-directionequal to (a) $100 \mathrm{Oe}$; (b) $185 \mathrm{Oe}$; (c) $300 \mathrm{Oe}$; (d) $400 \mathrm{Oe}$. The in-plane field opposes DMI field for DW propagating towards right and minimum displacement occurs at field equal to DMI field. Here $\mathrm{H}_{\mathrm{DMI}}=185-200$ Oe. 
Figure 6-12 shows the plot of the displacement (normalized) with respect to the applied inplane field. The plot shows a minimum at about 420 Oe. This can be considered as a rough estimate of HDMI. This shows that the DMI field is less than the applied field of 500 Oe in the experiments and corroborates with our DW velocity measurments. The DMI coefficient was

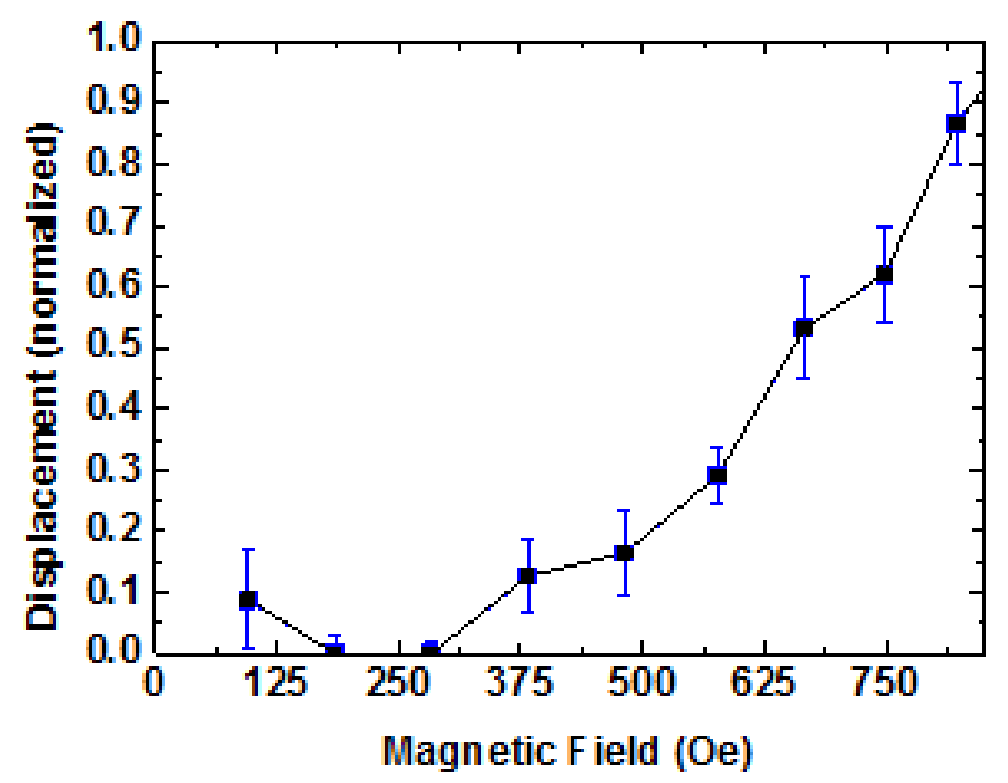

Figure 6-14 Domain wall displacement as a function of in-plane magnetic field. Minimum displacement corresponds to DMI field $=185-200$ Oe.

estimated from the equations provided in reference [37] and was found to be $0.35 \mathrm{~mJ} / \mathrm{m}^{2}$, the details are given in Appendix A6.

Similar measurements were repeated for stack with Pt spacer as $1 \mathrm{~nm}$ thick. The stack used in the measurements was $\mathrm{SiO}_{2} / \mathrm{Ta}(3) / \mathrm{Pt}(3) / \mathrm{Co}(0.7) / \mathrm{Pt}(1) / \mathrm{Co}(0.7) / \mathrm{Ta}(1)$. Figure 6-13 shows the Kerr imaging as in-plane magnetic field is gradually increased. The minimum displacement is obtained at around 185-200 Oe also plotted in Figure 6-14. Thus the $\mathrm{H}_{\mathrm{DMI}}$ field for thicker Pt spacer thickness $(=1 \mathrm{~nm})$ is roughly half of that when the Pt spacer thickness is less $(=0.5$ $\mathrm{nm})$. DMI coefficient was estimated as $0.19 \mathrm{~mJ} / \mathrm{m}^{2}$ and the details of calculations are provided in Appendix A6. It is worth noting the the field direction can also be reversed and 
corresponding displacement graphs can be obtained which would be shifted along the horizontal axis. The Pt thicknesses that we have used in our experiments has values $0.5 \mathrm{~nm}$ and $1 \mathrm{~nm}$. This thickness is low enough to cause ferrromagnetic coupling between the Co layers [40]. If the ferromagnetic layers were de-coupled or antiferromagnetically coupled we would observe double swithcing in the hysteresis loops which we do not observe. Hence the Co layers in our stack are ferromagnetically coupled.

\subsection{Summary}

SOT induced DW dynamics have been investigated in $\mathrm{Co} / \mathrm{Pt}$ double stack structures. $\mathrm{Pt} / \mathrm{Co} / \mathrm{Ta}$ thin film stacks are known to possess high SOT strength due to opposite signs of SHA of Ta and Pt. However the stacks were found to lose perpendicular anisotropy at higher temperature. A solution was proposed to this issue by adding additional $\mathrm{Co} / \mathrm{Pt}$ interface to limit the intermixing of Co and Ta. High DW speed in excess of $500 \mathrm{~m} / \mathrm{s}$ at a current density of $1 \times 10^{12}$ $\mathrm{A} / \mathrm{m}^{2}$ was observed in the wires fabricated from this stack. An in-plane field stabilized chiral Néel walls propagated along or against the current flow direction depending on the direction of the applied field. The hard-axis field aided in velocity enhancement due to prevention of Walker breakdown. The DW velocity along the current flow direction was larger as the external hard-axis field assisted the DMI field, whereas it was lower along the electron flow direction when the field opposed the DMI field. The DW velocity was found to increase with Ta capping thickness on account of higher SOT strength, and the velocity was found to decrease with increase in Pt spacer thickness on account of lower DMI and SOT strength from the bottom $\mathrm{Pt} / \mathrm{Co} / \mathrm{Pt}$ interface. Thus, double layer $\mathrm{Co} / \mathrm{Pt}$ stacks having enhanced thermal stability potentially leads towards new avenues of research in terms of high speed logic and memory applications. The high speed DW motion demonstrated in Co/Pt double stack Hall cross structures provides a template for an efficient memory device with potential high 
read/write speeds. Further improvement in DMI strength by tuning the heavy metal composition and thicknesses can eliminate the need of external field and also lower the driving current density. 


\section{Bibliography}

[1] D. A. Allwood, G. Xiong, C. C. Faulkner, D. Atkinson, D. Petit, and R. P. Cowburn, "Magnetic domain-wall logic," Science, vol. 309, pp. 1688-92, Sep 092005.

[2] A. Imre, G. Csaba, L. Ji, A. Orlov, G. H. Bernstein, and W. Porod, "Majority logic gate for magnetic quantum-dot cellular automata," Science, vol. 311, pp. 205-208, Jan 132006.

[3] S. S. P. Parkin, M. Hayashi, and L. Thomas, "Magnetic domain-wall racetrack memory," Science, vol. 320, pp. 190-194, Apr 112008.

[4] P. Xu, K. Xia, C. Z. Gu, L. Tang, H. F. Yang, and J. J. Li, "An all-metallic logic gate based on current-driven domain wall motion," Nature Nanotechnology, vol. 3, pp. 97-100, Feb 2008.

[5] L. Berger, "Emission of spin waves by a magnetic multilayer traversed by a current," Physical Review B, vol. 54, pp. 9353-9358, Oct 11996.

[6] J. C. Slonczewski, "Current-driven excitation of magnetic multilayers," Journal of Magnetism and Magnetic Materials, vol. 159, pp. L1-L7, Jun 1996.

[7] I. M. Miron, K. Garello, G. Gaudin, P. J. Zermatten, M. V. Costache, S. Auffret, et al., "Perpendicular switching of a single ferromagnetic layer induced by in-plane current injection," Nature, vol. 476, pp. 189-93, Aug 112011.

[8] L. Q. Liu, O. J. Lee, T. J. Gudmundsen, D. C. Ralph, and R. A. Buhrman, "Current-Induced Switching of Perpendicularly Magnetized Magnetic Layers Using Spin Torque from the Spin Hall Effect," Physical Review Letters, vol. 109, Aug 292012.

[9] L. Liu, C. F. Pai, Y. Li, H. W. Tseng, D. C. Ralph, and R. A. Buhrman, "Spin-torque switching with the giant spin Hall effect of tantalum," Science, vol. 336, pp. 555-8, May 042012.

[10] C. F. Pai, L. Q. Liu, Y. Li, H. W. Tseng, D. C. Ralph, and R. A. Buhrman, "Spin transfer torque devices utilizing the giant spin Hall effect of tungsten," Applied Physics Letters, vol. 101, Sep 172012.

[11] I. M. Miron, T. Moore, H. Szambolics, L. D. Buda-Prejbeanu, S. Auffret, B. Rodmacq, et al., "Fast current-induced domain-wall motion controlled by the Rashba effect," Nat Mater, vol. 10, pp. 419-23, Jun 2011.

[12] S. Emori, U. Bauer, S. M. Ahn, E. Martinez, and G. S. Beach, "Current-driven dynamics of chiral ferromagnetic domain walls," Nat Mater, vol. 12, pp. 611-6, Jul 2013.

[13] P. P. Haazen, E. Mure, J. H. Franken, R. Lavrijsen, H. J. Swagten, and B. Koopmans, "Domain wall depinning governed by the spin Hall effect," Nat Mater, vol. 12, pp. 299-303, Apr 2013.

[14] K. S. Ryu, L. Thomas, S. H. Yang, and S. Parkin, "Chiral spin torque at magnetic domain walls," Nature Nanotechnology, vol. 8, pp. 527-533, Jul 2013.

[15] J. E. Hirsch, "Spin Hall effect," Physical Review Letters, vol. 83, pp. 1834-1837, Aug 301999.

[16] M. Cubukcu, O. Boulle, M. Drouard, K. Garello, C. O. Avci, I. M. Miron, et al., "Spin-orbit torque magnetization switching of a three-terminal perpendicular magnetic tunnel junction," Applied Physics Letters, vol. 104, Jan 272014.

[17] K. Garello, I. M. Miron, C. O. Avci, F. Freimuth, Y. Mokrousov, S. Blugel, et al., "Symmetry and magnitude of spin-orbit torques in ferromagnetic heterostructures," Nature Nanotechnology, vol. 8, pp. 587-593, Aug 2013.

[18] J. Kim, J. Sinha, M. Hayashi, M. Yamanouchi, S. Fukami, T. Suzuki, et al., "Layer thickness dependence of the current-induced effective field vector in Ta vertical bar CoFeB vertical bar MgO," Nature Materials, vol. 12, pp. 240-245, Mar 2013.

[19] J. Torrejon, F. Garcia-Sanchez, T. Taniguchi, J. Sinha, S. Mitani, J. V. Kim, et al., "Currentdriven asymmetric magnetization switching in perpendicularly magnetized $\mathrm{CoFeB} / \mathrm{MgO}$ heterostructures," Physical Review B, vol. 91, Jun 292015.

[20] X. Fan, J. Wu, Y. P. Chen, M. J. Jerry, H. W. Zhang, and J. Q. Xiao, "Observation of the nonlocal spin-orbital effective field," Nature Communications, vol. 4, Apr 2013.

[21] Q. Hao and G. Xiao, "Giant Spin Hall Effect and Switching Induced by Spin-Transfer Torque in a W/Co40Fe40B20/MgO Structure with Perpendicular Magnetic Anisotropy," Physical Review Applied, vol. 3, Mar 262015.

[22] T. Moriya, "Anisotropic Superexchange Interaction and Weak Ferromagnetism," Physical Review, vol. 120, pp. 91-98, 1960. 
[23] I. E. Dzyaloshinskii, "Theory of Helicoidal Structures in Antiferromagnets .1. Nonmetals," Soviet Physics Jetp-Ussr, vol. 19, pp. 960-971, 1964.

[24] M. Heide, G. Bihlmayer, and S. Blugel, "Dzyaloshinskii-Moriya interaction accounting for the orientation of magnetic domains in ultrathin films: Fe/W(110)," Physical Review B, vol. 78, Oct 2008.

[25] S. H. Yang, K. S. Ryu, and S. Parkin, "Domain-wall velocities of up to $750 \mathrm{~m} \mathrm{~s}(-1)$ driven by exchange-coupling torque in synthetic antiferromagnets," Nature Nanotechnology, vol. 10, pp. 221-226, Mar 152015.

[26] P. J. Metaxas, J. Sampaio, A. Chanthbouala, R. Matsumoto, A. Anane, A. Fert, et al., "High domain wall velocities via spin transfer torque using vertical current injection," Scientific Reports, vol. 3, May 142013.

[27] S. Woo, M. Mann, A. J. Tan, L. Caretta, and G. S. D. Beach, "Enhanced spin-orbit torques in Pt/Co/Ta heterostructures," Applied Physics Letters, vol. 105, Nov 242014.

[28] X. Qiu, K. Narayanapillai, Y. Wu, P. Deorani, D. H. Yang, W. S. Noh, et al., "Spin-orbit-torque engineering via oxygen manipulation," Nat Nanotechnol, vol. 10, pp. 333-8, Apr 2015.

[29] A. W. J. Wells, P. M. Shepley, C. H. Marrows, and T. A. Moore. (2016, August 1, 2016). Effect of interfacial intermixing on the Dzyaloshinskii-Moriya interaction in $\mathrm{Pt} / \mathrm{Co} / \mathrm{Pt}$. ArXiv e-prints 1608. Available: http://adsabs.harvard.edu/abs/2016arXiv160803826W

[30] G. Q. Yu, P. Upadhyaya, K. L. Wong, W. J. Jiang, J. G. Alzate, J. S. Tang, et al., "Magnetization switching through spin-Hall-effect-induced chiral domain wall propagation," Physical Review $B$, vol. 89, Mar 252014.

[31] M. Hayashi, J. Kim, M. Yamanouchi, and H. Ohno, "Quantitative characterization of the spinorbit torque using harmonic Hall voltage measurements," Physical Review B, vol. 89, Apr 29 2014.

[32] A. V. Khvalkovskiy, V. Cros, D. Apalkov, V. Nikitin, M. Krounbi, K. A. Zvezdin, et al., "Matching domain-wall configuration and spin-orbit torques for efficient domain-wall motion," Physical Review B, vol. 87, Jan 42013.

[33] C. Hahn, G. de Loubens, O. Klein, M. Viret, V. V. Naletov, and J. Ben Youssef, "Comparative measurements of inverse spin Hall effects and magnetoresistance in YIG/Pt and YIG/Ta," Physical Review B, vol. 87, May 132013.

[34] G. Allen, S. Manipatruni, D. E. Nikonov, M. Doczy, and I. A. Young, "Experimental demonstration of the coexistence of spin Hall and Rashba effects in beta-tantalum/ferromagnet bilayers," Physical Review B, vol. 91, Apr 162015.

[35] M. Yamanouchi, D. Chiba, F. Matsukura, and H. Ohno, "Current-induced domain-wall switching in a ferromagnetic semiconductor structure," Nature, vol. 428, pp. 539-542, Apr 1 2004.

[36] D. Ravelosona, S. Mangin, J. A. Katine, E. E. Fullerton, and B. D. Terris, "Threshold currents to move domain walls in films with perpendicular anisotropy," Applied Physics Letters, vol. 90, Feb 122007.

[37] R. Lavrijsen, D. M. F. Hartmann, A. van den Brink, Y. Yin, B. Barcones, R. A. Duine, et al., "Asymmetric magnetic bubble expansion under in-plane field in $\mathrm{Pt} / \mathrm{Co} / \mathrm{Pt}$ : Effect of interface engineering," Physical Review B, vol. 91, Mar 172015.

[38] R. Soucaille, M. Belmeguenai, J. Torrejon, J. V. Kim, T. Devolder, Y. Roussigne, et al., "Probing the Dzyaloshinskii-Moriya interaction in $\mathrm{CoFeB}$ ultrathin films using domain wall creep and Brillouin light spectroscopy," Physical Review B, vol. 94, Sep 262016.

[39] K. S. Ryu, S. H. Yang, and S. Parkin, "Experimentally tunable chiral spin transfer torque in domain wall motion," New Journal of Physics, vol. 18, May 172016.

[40] M. Matczak, R. Schäfer, M. Urbaniak, B. Szymański, P. Kúwik, A. Jarosz, et al., "Domain wall generated by graded interlayer coupling in $\mathrm{Co} / \mathrm{Pt} / \mathrm{Co}$ film with perpendicular anisotropy," Applied Physics Letters, vol. 107, 2015. 


\section{Chapter 7}

\section{Conclusions and Outlook}

\subsection{Conclusions and Outcome}

This thesis presents experimental work on domain wall (DW) dynamics in in-plane magnetization anisotropy (IMA) and perpendicular magnetization anisotropy (PMA) nanostructures and nanostrips. To study the IMA system, $\mathrm{Ni}_{80} \mathrm{Fe}_{20}$ (permalloy) nanostructures have been employed due to their near zero crystalline anisotropy and high permeability. Thin films of permalloy are deposited on thermally oxidized silicon substrates by using ultra high vacuum dc magnetron sputtering technique and patterned into branch network structures using electron beam lithography and Ar-ion milling techniques. The branch network structures provide a system to examine the trajectory of transverse and vortex DWs as they propagate across the bifurcation. The DW dynamics in network structures find applications in studying the charge carrier hopping and magnetic monopole formation in artificial spin-ice structures, which are useful from fundamental standpoint. The network structures could also be used in designing magnetic logic devices as also demonstrated in this thesis.

The dimensions of the nanowire was kept around $120 \mathrm{~nm}$ to stabilize transverse DWs. A nucleation pad of larger dimension was patterned at one end to nucleate the DWs and a transverse nanowire was also placed to set the initial chirality of the injected DW. The trajectory of the injected DW across the bifurcation was found to be chirality dependent, e.g. head-to-head DW (HH-DW) with up-chirality propagated to the upper branch and HH-DW with down-chirality propagated to the lower branch. The direct observation of deterministic DW trajectory was observed using magnetic force microscopy (MFM) imaging. This was explained by the conservation and displacement of topological charges or defects during DW 
transformation process. The transverse DW transforms to a vortex configuration as the DW enters the bifurcation. The annihilation of the vortex DW at high field subsequently nucleates a transverse DW in one of the two branches. The motion of DW through the network displaces the edge defect at the bifurcation. This chirality dependent trajectory was successful when the distance of propagation was less than the DW fidelity length and the field was small. As the field increases the DW chirality oscillates due to Walker breakdown and the trajectory becomes stochastic. To overcome the issue of stochastic DW trajectory, a geometrical modification to the branch structure was proposed to confine the DW trajectory in one of the branches. The micromagnetic simulations and MFM imaging revealed the propagation of DW to the lower branch when the "u-shaped" branch was displaced along the $+y$-direction and vice-versa when the branch was displaced along the $-y$-direction. Minimum displacement or the offset of the branch was determined to induce the potential barrier which would constrain DW propagation along a particular branch. The nanowire dimensions were increased to $300 \mathrm{~nm}$ to stabilize the more robust vortex DW and "U-shaped" branch structure was selected with the object of using it to propose a logic device. The trajectory of the DW was found to be independent of the vortex core polarity or chirality.

The asymmetric "U-shaped" branch structure was employed to demonstrate a programmable logic device. The branch was displaced in the $+y$-direction to constrain the DW propagation along the lower branch. The programmability was achieved by fabricating a current carrying stripline at the bifurcation, overlapping with the horizontal nanowire, which served as a magnetic gate. A transverse nanowire was placed just before the bifurcation which served a dual purpose. The magnetization of the transverse nanowire provided one input bit and the shape anisotropy transformed vortex DW to a transverse DW just before it entered the branch. The transverse DW had a transverse charge distribution which allowed the DW trajectory to be controlled with the field provided by the magnetic gate. The magnetic gate 
overlapped with $25 \%$ of the nanowire to ensure that it only affected the DW whose base was facing it, since the majority of the charge of the DW is concentrated in the base. The second input was provided by the magnetization direction of the horizontal nanowire. The optimization of gate current and asymmetry barrier allowed different DW chiralities to propagate along different branch. For instance, to realize NAND gate operation, all DW chiralities except tailto-tail (TT) with down chirality propagate along the lower branch. The TT-DW with down chirality is attracted to the upper branch due to the direction of the gate current and the transverse charge distribution of the DW. Thus one branch gives the output of NAND gate while the output at the second branch is that of an AND gate. Two complimentary outputs are realized in one operation. By changing the current flow direction in the gate, NOR and OR gate operations can be realized. The logic operations were validated by MFM imaging and electrical detection of anisotropic magnetoresistance (AMR).

DW dynamics in PMA systems were investigated by fabricating Hall cross structures of $\mathrm{Co} / \mathrm{Ni}$ multilayer. $\mathrm{Co} / \mathrm{Ni}$ multilayers provide large perpendicular anisotropy and spin polarization which can be tuned by varying stack thicknesses. DW was nucleated by passing a current through a stripline, fabricated near one end of the nanowire, to generate local Oersted field. The DW was driven using either external out-of-plane field or by electric current utilizing spin-transfer torque (STT). The change in Hall resistance due to change in the magnetization when DW reaches it was observed by the phenomenon of anomalous Hall effect. Next, Hall cross structures with low anisotropy constant were fabricated and an in-plane pulsed current was applied to the nanowire. Multiple DWs were observed to nucleate without the application of local Oersted field. The DWs were detected by sweeping an external field and drop in Hall resistance was observed. Direct observation of multiple DWs were detected using Kerr microscopy technique. On increasing the current beyond a certain value the DWs were found to nucleate in the vicinity of Hall cross. The Hall cross provides a region of large 
demagnetization energy. The electrons near the vicinity of Hall cross are canted and this provides a natural anisotropy gradient. The in-plane pulsed current triggers the canted spins which may give rise to nucleation of DWs. The stochasticity in the nucleation process was used to demonstrate a random number generator device. The analog signal from the Hall sensor was fed to a band-pass amplifier and comparator built on a chip. A random stream of digital output was obtained.

Spin-orbit torque (SOT) induced DW dynamics were investigated in Co/Pt double stack. Reports indicate that $\mathrm{Pt} / \mathrm{Co} / \mathrm{Ta}$ stack exhibits enhanced SOT on account of opposite signs of spin Hall angle of Pt and Ta acting on opposite interfaces. However, we observed this stack loses PMA when annealed to high temperature on account of inter-diffusion of Ta and Co. We proposed a solution to this issue by inserting additional $\mathrm{Co} / \mathrm{Pt}$ interface in the stack which enhanced the PMA strength and thermal stability. Harmonic Hall measurements were performed to determine the strength of SOT or spin Hall angle of the proposed $\mathrm{Pt} / \mathrm{Co} / \mathrm{Pt} / \mathrm{Co} / \mathrm{Ta}$ stack. The spin Hall angle was found to enhance on increasing the Ta thickness and decrease on increasing the Pt spacer thickness due to competing effects of two Co/Pt interfaces. To estimate the strength of Dzyloshinskii-Moriya interaction (DMI) field, DW creep method was used by fabricating micron-strips and measuring the in-plane field required to minimize the DW velocity. Hall cross structures were patterned to estimate DW velocity by time of flight technique and verified by Kerr microscopy imaging technique. The DW velocity was found to increase when Ta thickness was increased due to enhancement in SOT and found to decrease when Pt spacer thickness was increased due to reduction in DMI and SOT. The velocity was found to be as high as $530 \mathrm{~m} / \mathrm{s}$ at an applied current density of $1 \times 10^{12} \mathrm{~A} / \mathrm{m}^{2}$. An in-plane field larger than DMI field was applied to prevent Walker breakdown and enhance the DW velocity. The DW velocity was found to be higher when external field was in the same direction as DMI field. The DW was found to propagate along the current flow direction opposite to that using 
STT. On reversing the field direction the DW velocity reduced since it opposed the DMI field and the direction of propagation also changed to electron flow direction. Thus, double layer $\mathrm{Co} / \mathrm{Pt}$ stacks having enhanced thermal stability potentially leads towards new avenues of research in terms of high speed logic and memory applications.

\subsection{Future Outlook}

Although the DW based racetrack memory is a strong candidate for universal memory due to its high read/write speeds and scalability, it suffers from some practical limitations. For instance, high current density is required to de-pin and move the DWs. There is a possibility of mutual annihilation of multiple DWs during motion leading to a reduction in packing density. The stray magnetic field from dipolar interaction between successive DWs also limits the packing density by affecting neighbouring DWs. The DW motion and spin polarization is material dependent. Solutions have been proposed to overcome these shortcomings which include patterning notches to control data flow, pulsed current to move the DW, using synthetic antiferromagnetic coupling to reduce the stray field [1]. More recently, heavy metal/ferromagnet/oxide stacks have been employed which include DMI field and SOT for efficient DW motion. This has reduced the current density for propagation [2-5]. In SOT, the anitdamping torque is always perpendicular to the magnetization, hence the incubation delay of the switching process is less which has led to high speed device performance [6]. Another advantage of SOT based driving is that the nanowire dimensions can be made longer to increase device density without suffering from high resistivity problem, since current flows through the heavy metal conductor. Recently, Lorentz transmission electron microscopy (L-TEM) imaging showed that existence of DMI makes the DWs topologically protected, preventing the walls from mutual annihilation [7]. This property would permit more DWs to be closely packed thereby increasing the device density. In the following sections we would propose extension 
of the work carried out in the thesis and highlight current and future research trends in magnetic devices.

\subsubsection{Programmable logic functionality}

The output of the DW based programmable logic device proposed in this thesis has been detected using anisotropic magnetoresistance (AMR) technique. The output can also be read using magnetic tunnel junction (MTJ) integrated with the device. The output branches can function as the free layer of the MTJ. The relative orientation of the magnetization between the free and fixed layer can be read as the change in the resistance of the sensor. Currently, the clocking is achieved via a global in-plane magnetic field. This can be generated locally by an on-chip inductor in the form of a meander to eliminate external magnetic field [8].

The device footprint can be optimized by overlaying two structures with a shared magnetic gate to realize a 3-D logic as shown in Figure 7-1. The advantage of such structure is that in one operation NAND, AND as well as NOR, OR outputs can be achieved. This is due to the opposite direction of magnetic fields experienced by the two devices. Although, the fabrication of this structure would be challenging, the two structures would need to be isolated by depositing a thin insulating layer.

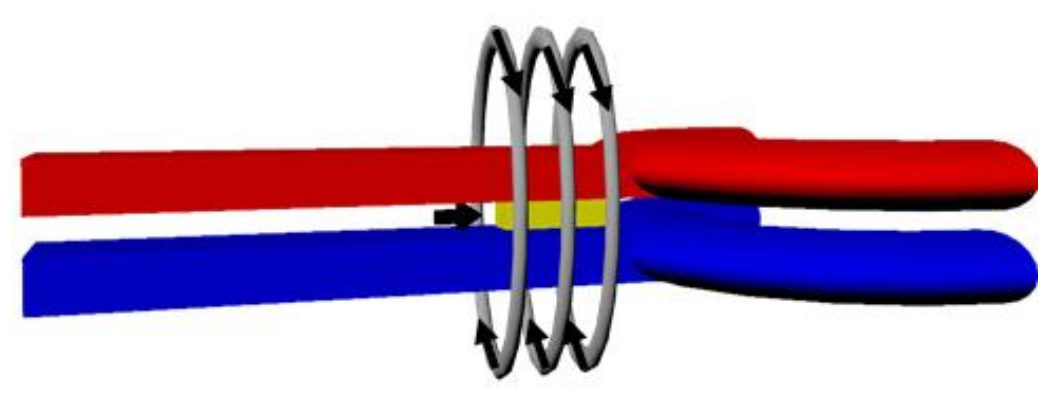

Figure 7-1 A schematic of 3-D logic scheme with a shared magnetic gate to realize NAND/AND and NOR/OR operations in the same run. 


\subsubsection{Spin-orbit torque induced domain-wall motion assisted by Dzyaloshinskii-Moriya interaction}

The high speed DW motion demonstrated in this thesis is a work in progress. The process recipe and stack composition can be further optimized to enhance DMI and spin Hall angle to facilitate efficient high speed DW motion. The increase in DMI would eliminate the requirement of applying hard-axis magnetic field. The sign of spin Hall angle could be changed to induce the DW motion along electron flow direction which would boost the speed from STT as well. Torrejon et al. [9], demonstrated the change in the sign of DMI and hence DW velocity with change in the thickness of the heavy metal underlayers. They showed than in $\mathrm{Ta} / \mathrm{CoFeB} / \mathrm{MgO}$ and $\mathrm{Hf} / \mathrm{CoFeB} / \mathrm{MgO}$ the $\mathrm{DW}$ would propagate along the electron flow direction and in $\mathrm{TaN} / \mathrm{CoFeB} / \mathrm{MgO}$ and $\mathrm{W} / \mathrm{CoFeB} / \mathrm{MgO}$ the $\mathrm{DW}$ would propagate along the current flow direction for certain thickness of the heavy metal layer. However, the DW velocity obtained was less than $20 \mathrm{~m} / \mathrm{s}$.

In this thesis, the DW dynamics were explored for Co/Pt double stack with Pt underlayer. If Pt is replaced with Ta, the sign of spin Hall angle would change and DW would propagate along the electron flow direction under the influence of both STT and SOT. However, we were unable to obtain perpendicular magnetic anisotropy (PMA) with Ta underlayer, necessitating the need of Pt as an underlayer for Co to induce PMA. We can investigate alternate heavy metal underlayers for instance $\mathrm{Hf}$ and $\mathrm{W}$, which was recently reported to have large spin Hall angle [10]. The speed can also be increased by lowering the anisotropy of the nanowire [11]. This can be achieved by increasing the thickness of the magnetic layers as was shown in Chapter 5 . However, increasing the thickness would also lower the strength of DMI since it is an interfacial phenomenon. Thus the thickness needs to be optimized to lower the anisotropy while maintaining high DMI strength. High DMI strength increases the short range repulsion between neighbouring DWs preventing mutual annihilation and allowing higher density of DWs. This 
was demonstrated by Real et al. [12], where they showed Néel DWs forming coupled state in the presence of out-of-plane magnetic field, however, their current driven velocity was independent of the applied field. This property allows highly compressed DWs to be driven by current pulses. In our stack, to enhance the DMI strength the symmetry of the stack can be broken by adding an oxide layer such as $\mathrm{MgO}$ or $\mathrm{AlO}_{\mathrm{x}}$ Yang et al. [13] reported through first principle calculations that $\mathrm{Pt} / \mathrm{Co} / \mathrm{MgO}$ stack has DMI strength which is 1.6 times that of $\mathrm{Pt} / \mathrm{Co}$ bilayer. The oxygen incorporation can also enhance DMI as was reported by Qiu et al. [14]. Recent reports also suggest Pt/Co/Ir stack to have much larger DMI than Pt/Co bilayer [13]. This is due to opposite orientation of DMI vector at the $\mathrm{Co} / \mathrm{Pt}$ and $\mathrm{Co} / \mathrm{Ir}$ interfaces which add up since they are acting on opposite interfaces. DW dynamics can be explored in $\mathrm{Pt} / \mathrm{Co} / \mathrm{Ir}$ stacks.

Field free switching and DW motion can be achieved by integrating the device with antiferromagnetic layers. Recent studies [15, 16] indicate field-free SOT induced magnetization switching of ferromagnetic layers in contact with antiferromagnetic layer. The switching is induced by utilizing the exchange bias field originating from the antiferromagnetic layer. Yang et al. [1] demonstrated DW velocities in excess of $750 \mathrm{~m} / \mathrm{s}$ along the current flow direction utilizing the SOT along with exchange coupling torque from synthetic antiferromagnetically coupled $\mathrm{Co} / \mathrm{Ni}$ multilayers. The use of this concept can be extended to study alternate magnetic stacks for instance $\mathrm{Co} / \mathrm{Pt}$ multilayer which have larger perpendicular anisotropy. The interplay of different torque mechanism can be explored in detail. 


\subsubsection{Voltage controlled magnetization switching and domain-wall motion}

For device applications, it is essential to locally control the DW motion which cannot be achieved using external magnetic field and current induced DW motion. Application of an electric field perpendicular to the plane of a PMA material can achieve this control by modification of the anisotropy $[17,18]$. This technique is advantageous since it involves very low power for switching operation [19]. Theoretical reports suggest that the anisotropy can be altered if the surface states are modified by the application of electric field at the ferromagnet/oxide interface [20]. Schellekens et al. [19] considered $\mathrm{Pt} / \mathrm{Co} / \mathrm{AlO}_{\mathrm{x}}$ thin film stack and demonstrated dependence of DW velocity on the magnitude and sign of the applied voltage across the ferromagnetic layer. Recently, Bauer et al. [21] applied voltage controlled magnetization switching to create DW traps in $\mathrm{Pt} / \mathrm{Co} / \mathrm{GdO}_{\mathrm{x}}$ structures. $\mathrm{GdO}_{\mathrm{x}}$ has a high dielectric constant, which would lead to larger charging effects at the interface and hence enhanced modification of PMA. The researchers attributed the strong influence on DW trajectory to have ionic rather than electronic origin, due to the property of $\mathrm{GdO}_{\mathrm{x}}$ being a strong ionic conductor. The $\mathrm{O}^{2-}$ vacancy transport in the $\mathrm{GdO}_{\mathrm{x}}$ permits voltage controlled $\mathrm{O}^{2-}$ accumulation or depletion at the $\mathrm{Co} / \mathrm{GdO}_{\mathrm{x}}$ interface. Since the PMA in $\mathrm{Co} / \mathrm{Oxide}$ interfaces depends on the Co-O coordination, the voltage would lead to modification of anisotropy and thereby creating DW traps. This property can be used to realize bit selection in racetrack memory shift registers.

Voltage-controlled racetrack memory could eliminate the need of notches and other geometrical pinning sites, thereby reducing the current and power required to de-pin the DW from these sites. The device could potentially employ electric gate to modulate the flow of DW providing greater control. However, in such systems the velocity of DW is low $(0.1 \mathrm{~m} / \mathrm{s})$, limited by thermally activated creep regime. The only experimental study reported for electric 


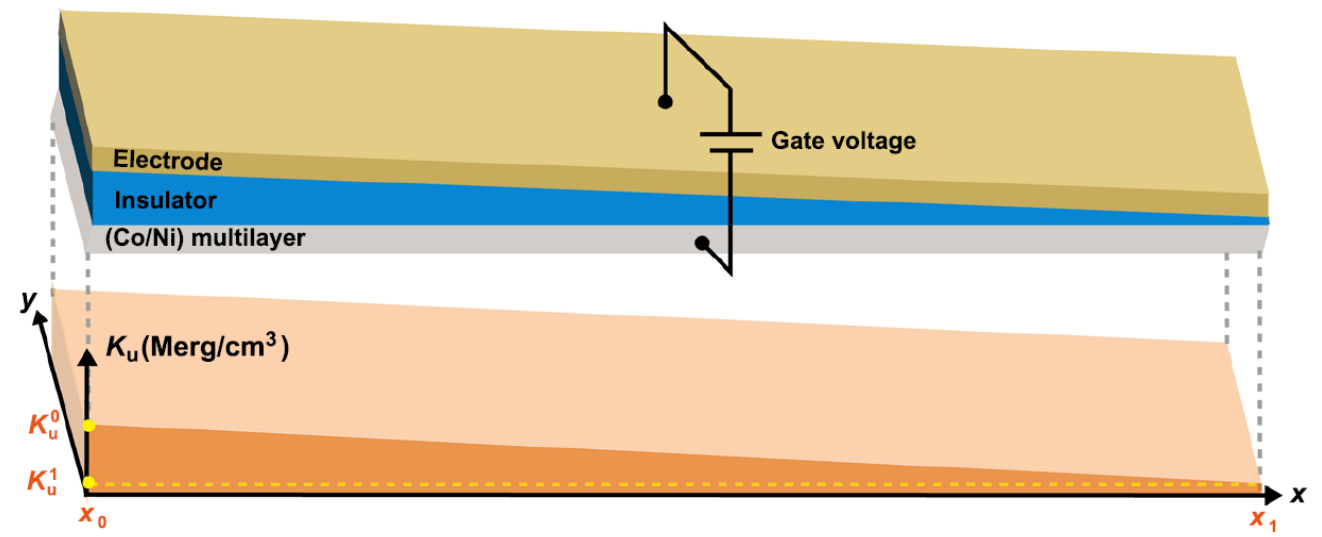

Figure 7-2 Schematic to illustrate sloped electric field in stack for domain-wall propagation. Adapted from [22].

field induced motion in Walker regime was carried out by Lin et al. [23]. The velocity of 20 $\mathrm{m} / \mathrm{s}$ was reported on $\mathrm{Ta} / \mathrm{CoFeB} / \mathrm{MgO} / \mathrm{TiO}_{2} / \mathrm{ITO}$ structures, by applying voltage between bottom $\mathrm{Ta} / \mathrm{CoFeB}$ electrode and the top indium tin oxide (ITO) electrode. It is worth noting that an external magnetic field is still needed to induce the motion. No experimental studies report the sole use of electric field to induce the DW motion. Yamada et al. [22] showed through micromagnetic simulations that presence of sloped electric field could lead to DW motion without the need of external magnetic field. The sloped electric field is provided by gradually varying insulator thickness across the stack, to give it a wedge shape as shown in Figure 7-2. The anisotropy of the stack also varies along the length of the stack. We can demonstrate experimental prototype of such structure which could pave the way forward for high density and low power domain wall based memory and logic devices. Essentially combination of high DMI, STT, SOT and voltage induced DW dynamics is the key to realizing universal DW based memory and logic devices. 


\section{Bibliography}

[1] S. H. Yang, K. S. Ryu, and S. Parkin, "Domain-wall velocities of up to $750 \mathrm{~m} \mathrm{~s}(-1)$ driven by exchange-coupling torque in synthetic antiferromagnets," Nature Nanotechnology, vol. 10, pp. 221-6, Mar 2015.

[2] S. Emori, U. Bauer, S. M. Ahn, E. Martinez, and G. S. Beach, "Current-driven dynamics of chiral ferromagnetic domain walls," Nat Mater, vol. 12, pp. 611-6, Jul 2013.

[3] P. P. Haazen, E. Mure, J. H. Franken, R. Lavrijsen, H. J. Swagten, and B. Koopmans, "Domain wall depinning governed by the spin Hall effect," Nat Mater, vol. 12, pp. 299-303, Apr 2013.

[4] K. S. Ryu, L. Thomas, S. H. Yang, and S. Parkin, "Chiral spin torque at magnetic domain walls," Nature Nanotechnology, vol. 8, pp. 527-533, Jul 2013.

[5] D. Bhowmik, "Spin orbit torque driven magnetic switching for low power computing and memory," Ph. D. Thesis, Electrical Engineering and Computer Sciences, University of California at Berkeley, 2015.

[6] K. Garello, C. O. Avci, I. M. Miron, M. Baumgartner, A. Ghosh, S. Auffret, et al., "Ultrafast magnetization switching by spin-orbit torques," Applied Physics Letters, vol. 105, 2014.

[7] M. J. Benitez, A. Hrabec, A. P. Mihai, T. A. Moore, G. Burnell, D. McGrouther, et al., "Magnetic microscopy and topological stability of homochiral Neel domain walls in a Pt/Co/AlOx trilayer," Nature Communications, vol. 6, Dec 2015.

[8] W. C. Jeong, J. H. Park, J. H. Oh, G. T. Jeong, H. S. Jeong, and K. Kim, "Highly scalable MRAM using field assisted current induced switching," in Digest of Technical Papers Symposium on VLSI Technology, 2005, pp. 184-185.

[9] J. Torrejon, J. Kim, J. Sinha, S. Mitani, M. Hayashi, M. Yamanouchi, et al., "Interface control of the magnetic chirality in $\mathrm{CoFeB} / \mathrm{MgO}$ heterostructures with heavy-metal underlayers," Nature Communications, vol. 5, Aug 2014.

[10] Q. Hao and G. Xiao, "Giant Spin Hall Effect and Switching Induced by Spin-Transfer Torque in a W/Co40Fe40B20/MgO Structure with Perpendicular Magnetic Anisotropy," Physical Review Applied, vol. 3, Mar 262015.

[11] D. H. Kim, S. C. Yoo, D. Y. Kim, K. W. Moon, S. G. Je, C. G. Cho, et al., "Maximizing domain-wall speed via magnetic anisotropy adjustment in $\mathrm{Pt} / \mathrm{Co} / \mathrm{Pt}$ films," Applied Physics Letters, vol. 104, 2014.

[12] R. P. Del Real, V. Raposo, E. Martinez, and M. Hayashi, "Current-Induced Generation and Synchronous Motion of Highly Packed Coupled Chiral Domain Walls," Nano Letters, vol. 17, pp. 1814-1818, 2017.

[13] H. Yang, O. Boulle, V. Cros, A. Fert, and M. Chshiev, "Controlling Dzyaloshinskii-Moriya Interaction via Chirality Dependent Layer Stacking, Insulator Capping and Electric Field," ArXiv e-prints, vol. 1603, p. arXiv:1603.01847, 2016.

[14] X. Qiu, K. Narayanapillai, Y. Wu, P. Deorani, D. H. Yang, W. S. Noh, et al., "Spin-orbit-torque engineering via oxygen manipulation," Nat Nanotechnol, vol. 10, pp. 333-8, Apr 2015.

[15] S. Fukami, C. Zhang, S. Duttagupta, A. Kurenkov, and H. Ohno, "Magnetization switching by spin-orbit torque in an antiferromagnet-ferromagnet bilayer system," Nature Materials, vol. 15, pp. 535-541, 2016.

[16] Y. W. Oh, S. H. C. Baek, Y. M. Kim, H. Y. Lee, K. D. Lee, C. G. Yang, et al., "Field-free switching of perpendicular magnetization through spin-orbit torque in antiferromagnet/ferromagnet/oxide structures," Nature Nanotechnology, vol. 11, pp. 878-884, 2016.

[17] M. Weisheit, S. Fähler, A. Marty, Y. Souche, C. Poinsignon, and D. Givord, "Electric fieldinduced modification of magnetism in thin-film ferromagnets," Science, vol. 315, pp. 349-351, 2007.

[18] D. Chiba, S. Fukami, K. Shimamura, N. Ishiwata, K. Kobayashi, and T. Ono, "Electrical control of the ferromagnetic phase transition in cobalt at room temperature," Nature Materials, vol. 10, pp. 853-856, 2011. 
[19] A. J. Schellekens, A. Van Den Brink, J. H. Franken, H. J. M. Swagten, and B. Koopmans, "Electric-field control of domain wall motion in perpendicularly magnetized materials," Nature Communications, vol. 3, 2012.

[20] M. K. Niranjan, C. G. Duan, S. S. Jaswal, and E. Y. Tsymbal, "Electric field effect on magnetization at the $\mathrm{Fe} / \mathrm{MgO}(001)$ interface," Applied Physics Letters, vol. 96, 2010.

[21] U. Bauer, S. Emori, and G. S. D. Beach, "Voltage-controlled domain wall traps in ferromagnetic nanowires," Nature Nanotechnology, vol. 8, pp. 411-416, Jun 2013.

[22] K. Yamada, S. Murayama, and Y. Nakatani, "Magnetic domain wall motion in Co/Ni nanowires induced by a sloped electric field," Applied Physics Letters, vol. 108, May 162016.

[23] W. W. Lin, N. Vernier, G. Agnus, K. Garcia, B. Ocker, W. S. Zhao, et al., "Universal domain wall dynamics under electric field in $\mathrm{Ta} / \mathrm{CoFeB} / \mathrm{MgO}$ devices with perpendicular anisotropy," Nature Communications, vol. 7, Nov 162016. 


\section{Appendix A}

\section{A.1 X-ray photoelectron spectroscopy of Co/Pt double stack}

$\mathrm{X}$-ray photoelectron spectroscopy (XPS) measurements were performed on the Co/Pt double stack with the following stack composition: Ta (3)/Pt (3)/Co (0.7)/Pt (0.5)/Co (0.7)/Ta (3)/Pt (1), where thicknesses are in nm. Figure A.1-1 (a-c) shows the spectra of Pt, Ta and Co respectively with their respective binding energies. Clearly $\mathrm{TaOx}$ is not present and $\mathrm{CoO}$ is not formed due to the absence of satellite at $786 \mathrm{eV}$. Figure A.1-2 below shows the atomic concentration depth profile of the multilayer stack. The middle region $\mathrm{Co} / \mathrm{Pt} / \mathrm{Co}$ shows almost overlap between the Co layers, since the Pt thickness involved is very low $(0.5 \mathrm{~nm})$ which is less than sputter rate used in the XPS at $45^{\circ}$ angle $(0.51 \mathrm{~nm} / \mathrm{s})$. It can be masked by the neighboring strong Pt signal. Also the surface roughness could lead to reduction in signal strength. Moreover, we observe that Pt concentration keeps going higher from the middle portion of $\mathrm{Co} / \mathrm{Pt} / \mathrm{Co}$ indicating that $\mathrm{Pt}$ is indeed present. Since the measurement area is $200 \mu \mathrm{m}$ $\times 200 \mu \mathrm{m}$, we cannot comment on the continuity of Pt from these measurements. The XPS sampling depth is less than $0.5-2 \mathrm{~nm}$ for metal, in order to reduce its effect, the take off-angle is reduced to 10 degree in these measurements to reduce etch rate. 
(a)

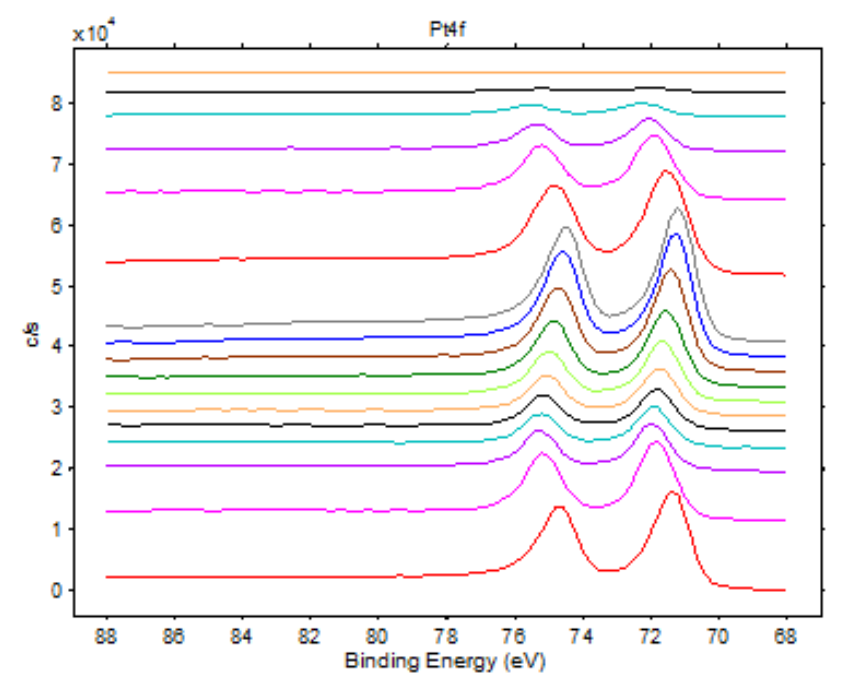

(b)
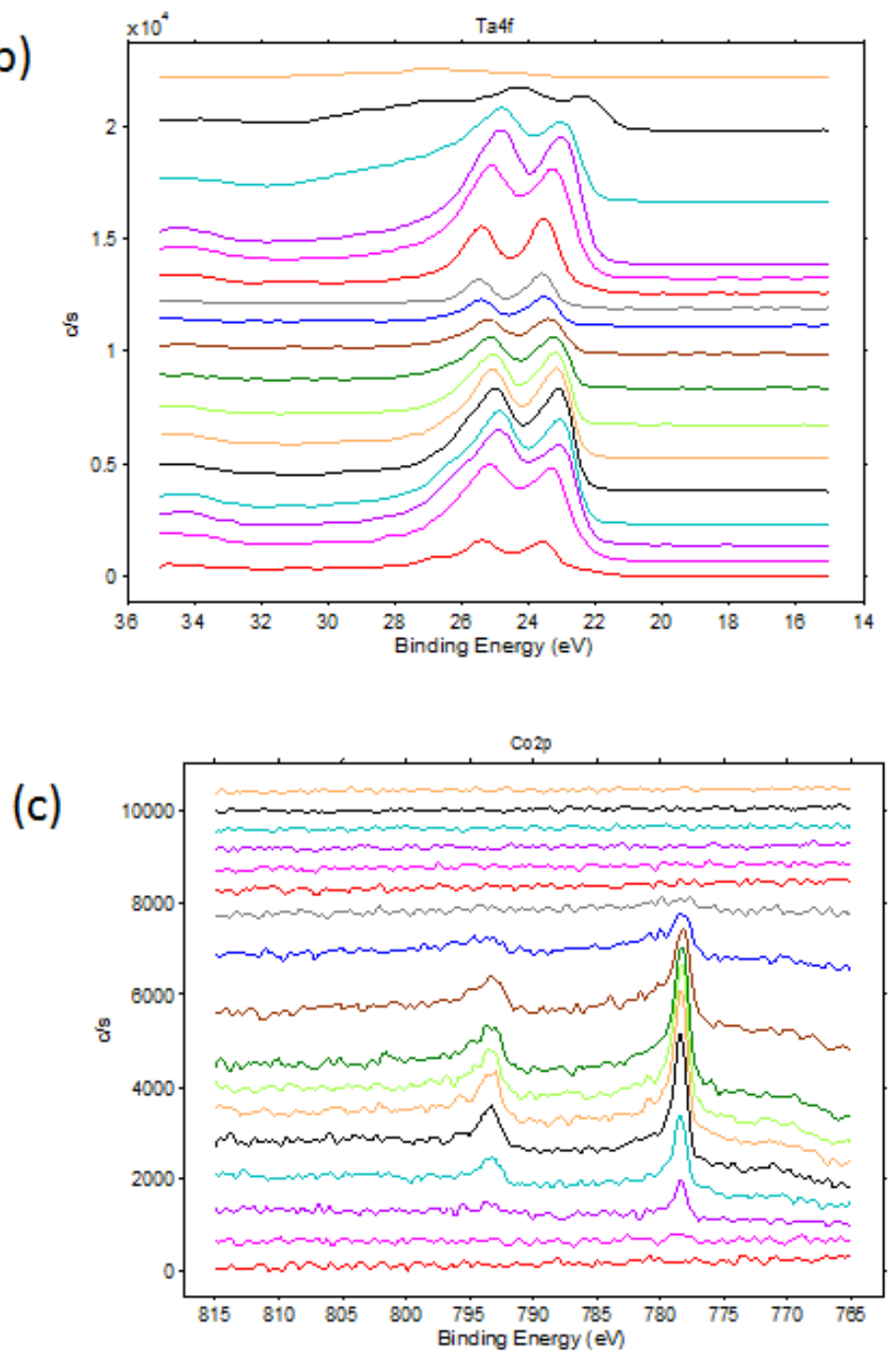

Figure A.1-1. The spectrum of elements and their binding energies obtained using XPS, (a) Pt, (b) Ta and (c) Co. 


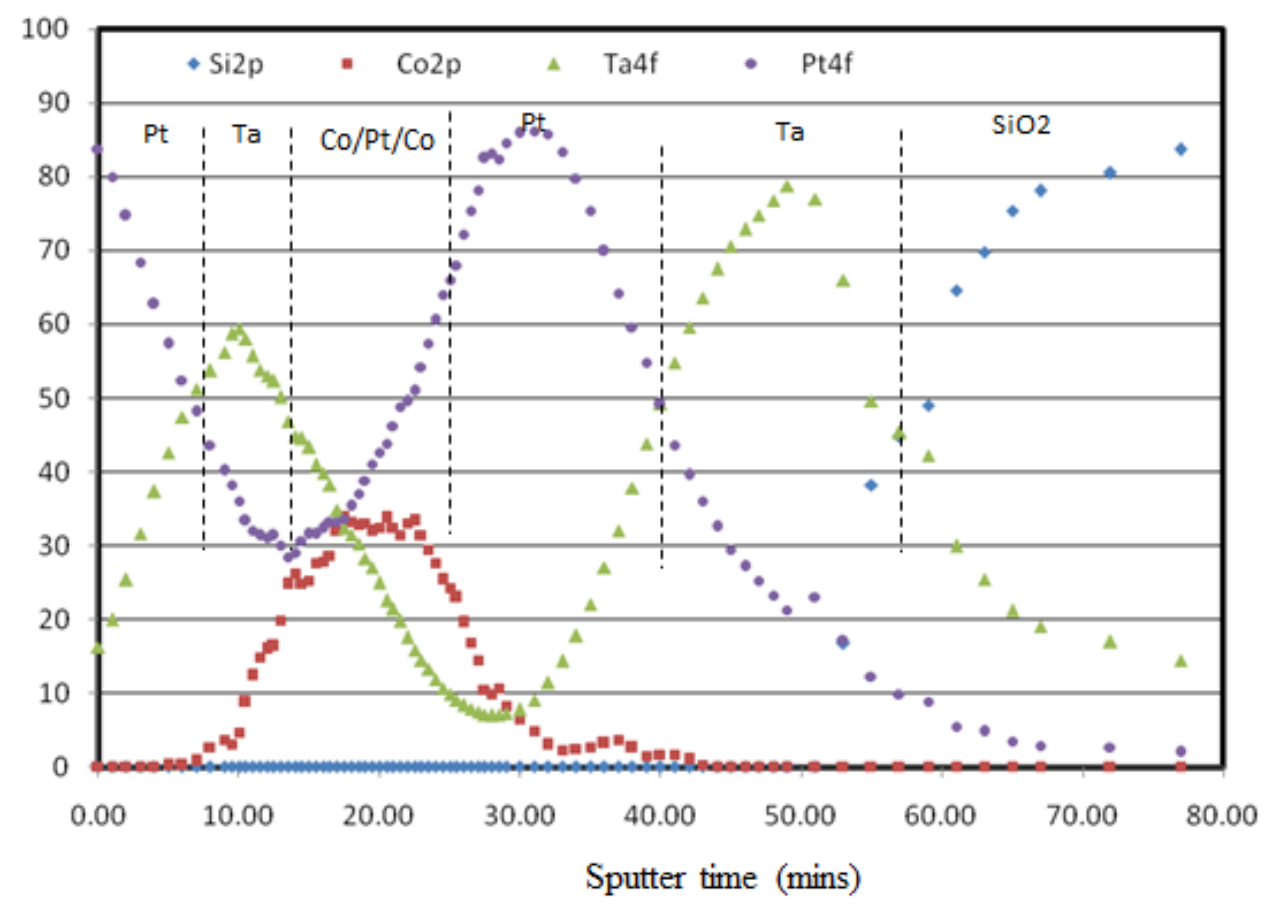

Figure A.1-2. Atomic concentration of depth profile obtained by XPS measurements for the $\mathrm{Co} / \mathrm{Pt}$ double stack.

\section{A2. Determination of anisotropy constant of $\mathrm{Co} / \mathrm{Pt}$ double stack}

The out-of-plane anisotropy constant, $\mathrm{K}_{\mathrm{u}}$ was estimated from the relation, $\mathrm{K}_{\mathrm{u}}=\mathrm{M}_{\mathrm{S}} \mathrm{H}_{\mathrm{K}} / 2$, where $\mathrm{M}_{\mathrm{S}}$ is the saturation magnetization and $\mathrm{H}_{\mathrm{K}}$ is the hard-axis saturation field or the field at which the PMA sample saturates with the application of in-plane external field. Figure A2 (a) shows the M-H loops for original thin film sample having the composition Ta (3)/Pt (3)/Co (0.7)/Pt $(0.5) / \mathrm{Co}(0.7) / \mathrm{Ta}$ (1). Figure A2 (b) shows the plots when Pt spacer thickness is increased from $0.5 \mathrm{~nm}$ to $1 \mathrm{~nm}$ keeping other thicknesses fixed. Figure A2 (c) shows the plots when Ta capping thickness is increased from $1 \mathrm{~nm}$ to $3 \mathrm{~nm}$ keeping Pt spacer thickness as $0.5 \mathrm{~nm}$.

The plot in Figure A2 (a) is magnified to show clear square hystersis of the out-of-plane MH loop. The vertical line intersects the $x$-axis at the corresponding $\mathrm{H}_{\mathrm{K}}$ values, which are 4000, 

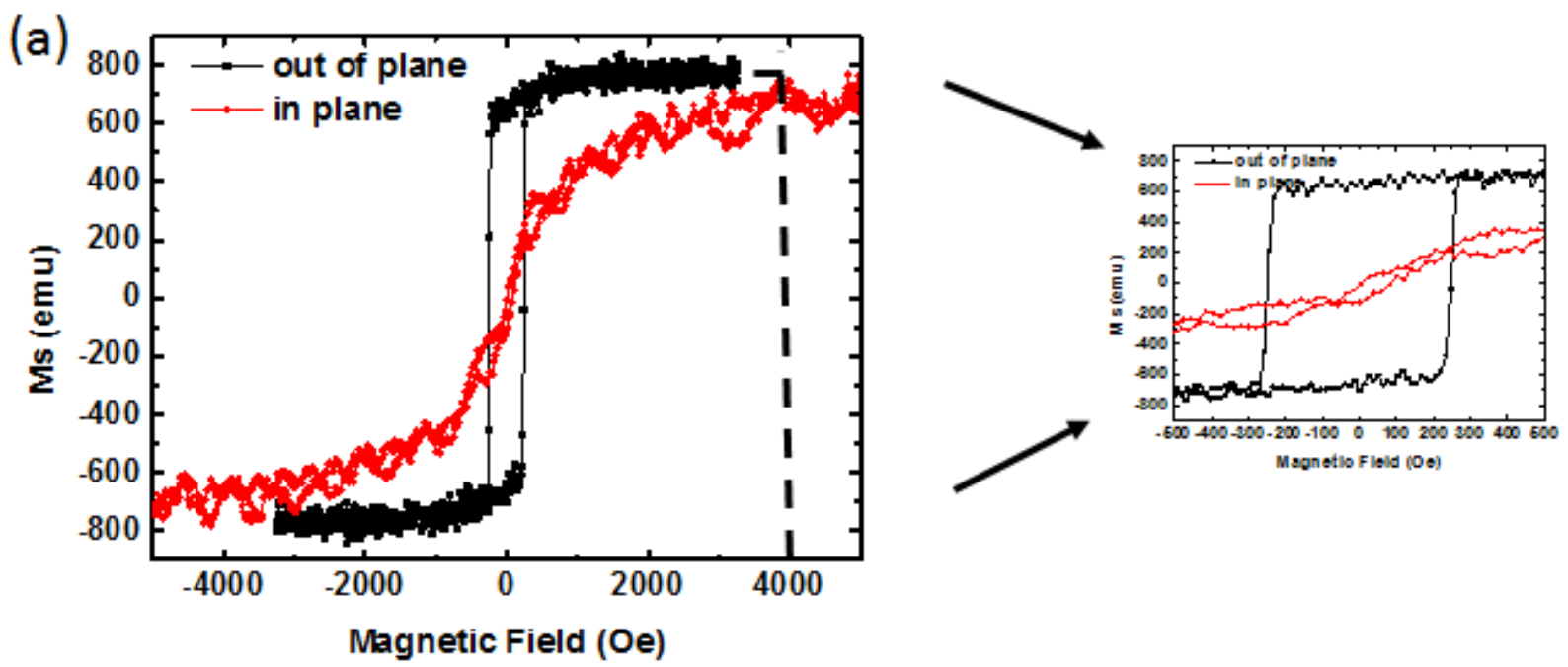

(b)

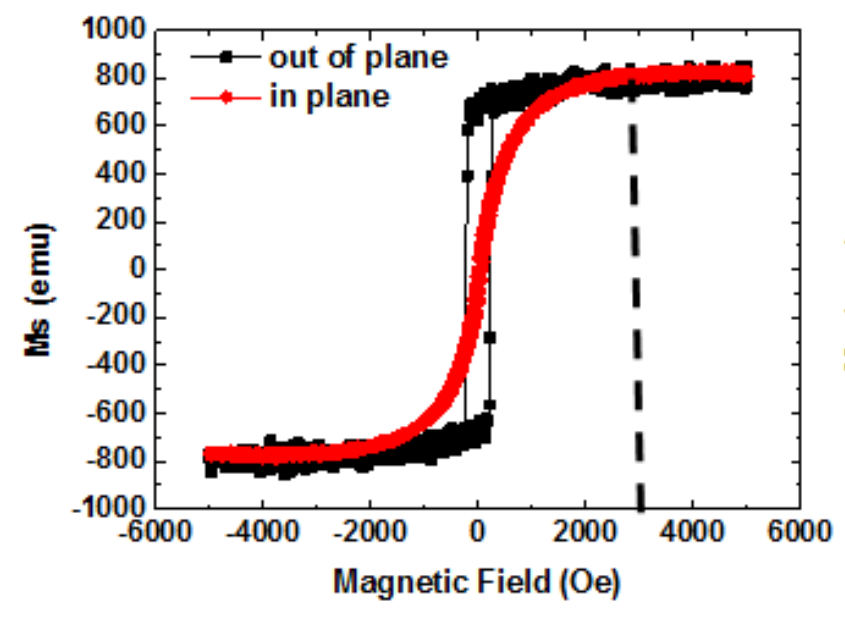

(c)

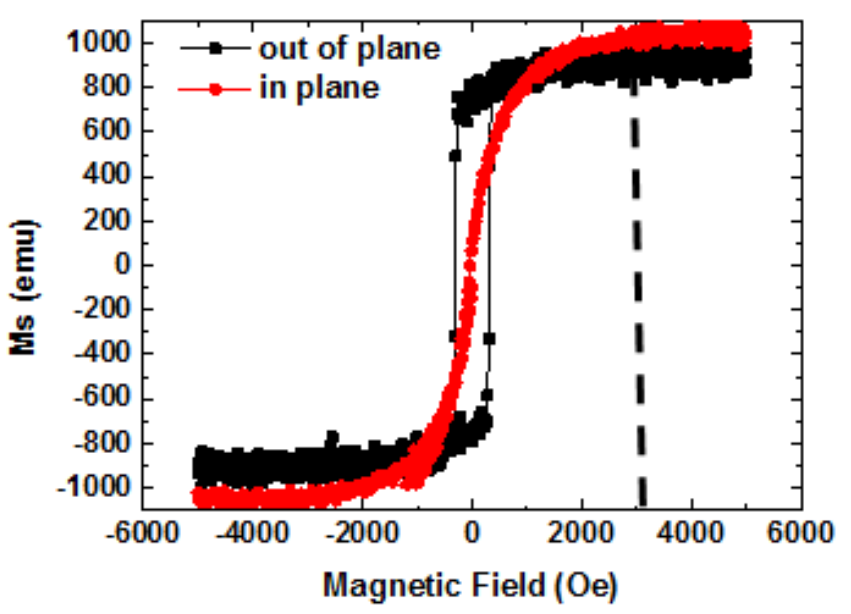

Figure A2. AGFM measurements of thin film stack for estimation of anisotropy constant. (a) Thin film stack Ta (3)/Pt (3)/Co (0.7)/Pt (0.5)/Co (0.7)/Ta (1). The insset shows a magnified view of the out-of-plane $\mathrm{M}-\mathrm{H}$ loop to indicate square hysteresis (b) Pt spacer thickness increased, Ta (3)/Pt (3)/Co (0.7)/Pt (1)/Co (0.7)/Ta (1). (c) Ta thickness increased Ta (3)/Pt (3)/Co $(0.7) / \mathrm{Pt}(0.5) / \mathrm{Co}(0.7) / \mathrm{Ta}(3)$.

3000 and 3000 Oe respectively for the three thin films. The $\mathrm{M}_{\mathrm{S}}$ for three thin films are respectively, 800, 820 and $900 \mathrm{emu} / \mathrm{cc}$. The $\mathrm{K}_{\mathrm{U}}$ for the three thin films are respectively, $1.6 \times 10^{6}, 1.23 \times 10^{6}$ and $1.35 \times 10^{6} \mathrm{ergs} / \mathrm{cc}$. 


\section{A3. Effect of in-plane field on domain wall motion and structure}

In the presence of an in-plane field, the DW would be canted and have some tilt. It would have a Néel component. It would not be a perfect Bloch wall even if the width of the nanowire is large. If the DMI is present indeed the structure would be perfect Neel and in-plane field would either support or oppose the motion. Figure A3 shows the simulations performed using MuMag simulation tool $[1,2]$. The simulation parameters are $\mathrm{M}_{\mathrm{S}}=800 \times 10^{3} \mathrm{~A} / \mathrm{m}, \mathrm{K}_{\mathrm{u}}=5 \times 10^{5} \mathrm{~J} / \mathrm{m}^{3}, \mathrm{~A}$ $=18 \mathrm{pJ} / \mathrm{m}$ from reference [3], the spin Hall angle is 0.15 , DMI coefficient, D $=0.3 \mathrm{~mJ} / \mathrm{m}^{2}$. These are typical parameters observed experimentally for $\mathrm{Co} / \mathrm{Pt}$ double stack. $\mathrm{K}_{\mathrm{u}}$ is obtained by adding demagnetization term to $K_{\text {eff }}$ obtained in A2. Thus $K_{u}=K_{\text {eff }}+\mu_{0} M_{s}^{2} / 2$.

Figure A3 (a)-(i) shows the initial simulated configuration at time $\mathrm{t}=0$, when a small Dzyaloshinskii-Moriya interaction (DMI), D = $0.3 \mathrm{~mJ} / \mathrm{m}^{2}$ stablilizes a left handed Neel wall. A current is applied along the $+x$-direction. An in-plane field $=500$ Oe was applied along the $x$-direction to assist the left handed chirality. Figure A3 (a)-(ii) shows the final simulated configuration at a finite time. The DW propagates along the current flow direction due to SOT and while in motion, the spins adopt a chirality which is partially Neel and partially Bloch.

Figure A3 (b)-(i) shows the initial simulated configuration at time $t=0$, when a small Dzyaloshinskii-Moriya interaction (DMI), D $=0.3 \mathrm{~mJ} / \mathrm{m}^{2}$ stablilizes a left handed Neel wall. A current is applied along the $+x$-direction. An in-plane field $=500$ Oe is now applied along the $+x$-direction to oppose the left handed chirality. Figure A3 (b)-(ii) shows the final simulated configuration at a finite time. The DW propagates against the current flow direction as shown in our experiments. While in motion, the spins adopt a chirality which is Bloch, hence the motion is along the electron flow direction. In this case the in-plane field is able to overcome the DMI strength. 


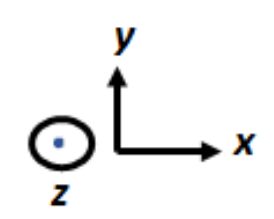

(i)

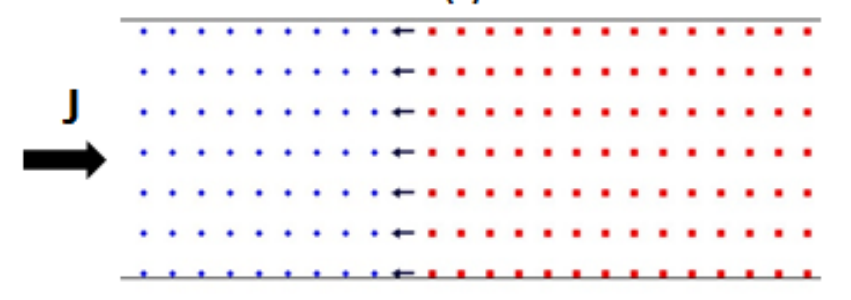

\section{In-plane field $=\mathbf{5 0 0}$ Oe}

(a)

(ii)

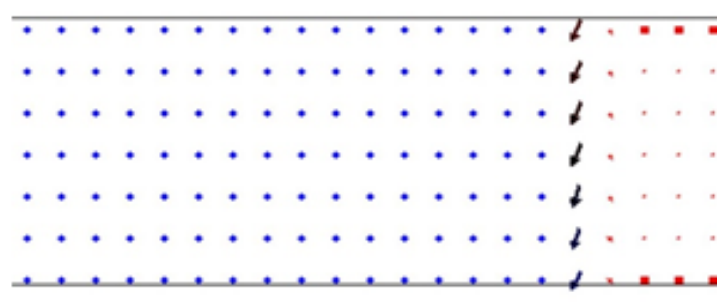

(b)

(i)

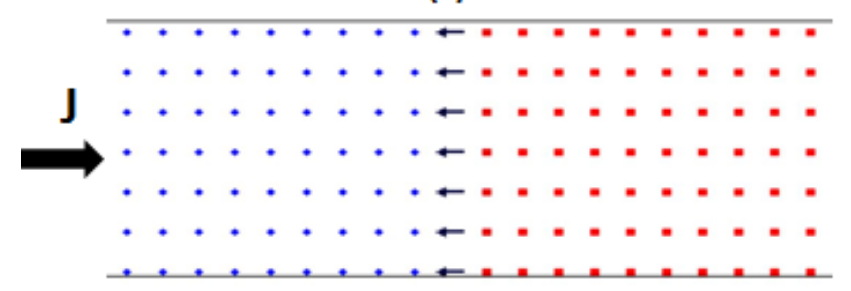

(ii)

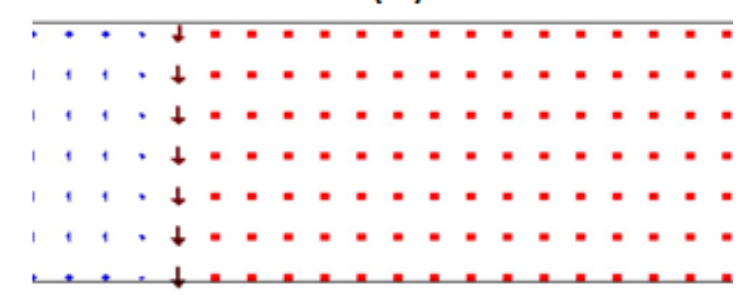

In-plane field = 500 Oe

Figure A3. Micromagnetic simulations to show the effect of in-plane field on DW motion. Current is applied along $+x$-direction. DMI, $\mathrm{D}=0.3 \mathrm{~mJ} / \mathrm{m}^{2}$ (a) Field $=500$ Oe along $-x$ direction (i) Initial configuration (ii) Final configuration. (b) Field $=500 \mathrm{Oe}$ along $+x$-direction (i) Initial configuration (ii) Final configuration.

\section{A4. Domain wall velocities: Down-Up chirality}

Figure A4-1 (a) shows the results of domain wall (DW) velocity with applied current density, the DW being nucleated in a down-up chirality. The results are similar to the ones shown in Figure 6-8 (c) with the difference being that magnetic field in the $+x$-direction now favours DW motion along the current flow and opposite is the case with magnetic field in the $-x$ direction. Only slight differences in the magnitude of the velocities were observed. The other 
observations are similar to the ones reported for Figure 6-8 (c). Figure A4-1 (b) shows the results when Ta thickness is increased to $3 \mathrm{~nm}$ and Figures A4-1 (c-d) show the comparison.

Figure A4-2 shows the plot when Pt spacer thickness is increased. The observations are similar to the ones reported in Figure 4 with the in-plane field direction reversed. Only slight differences in the magnitude of the velocities were observed.

(a)

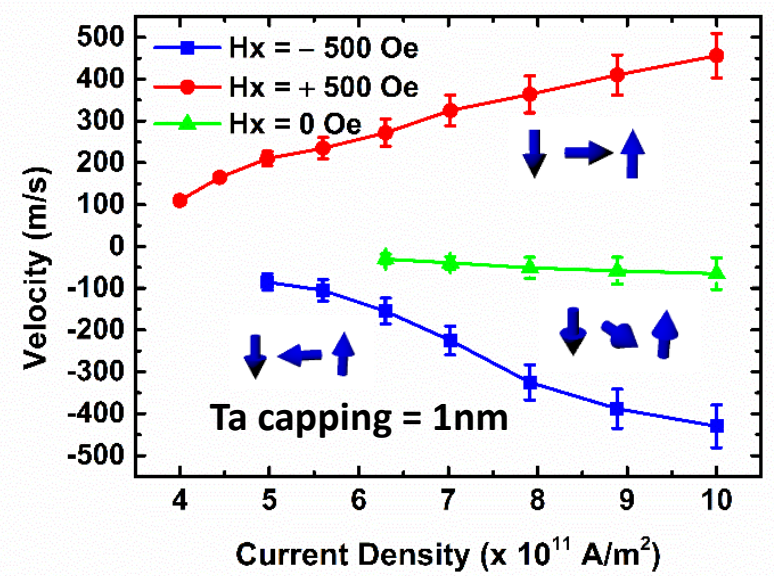

(c)

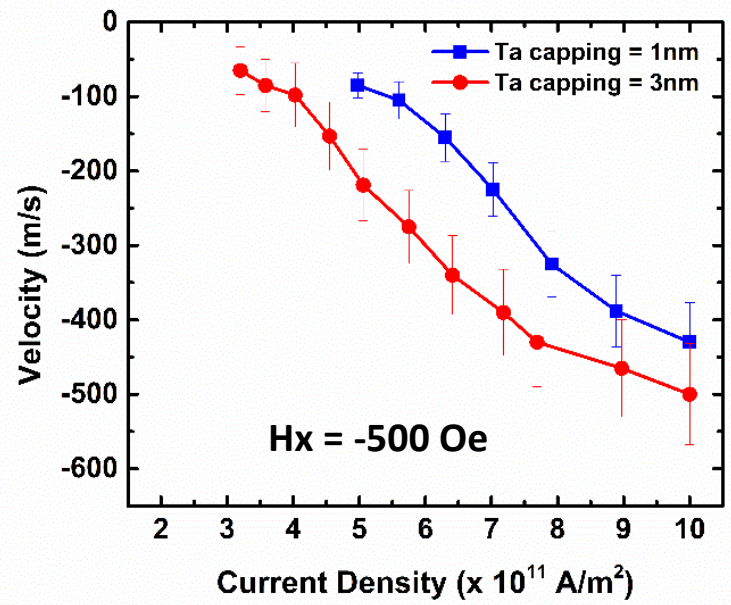

(b)

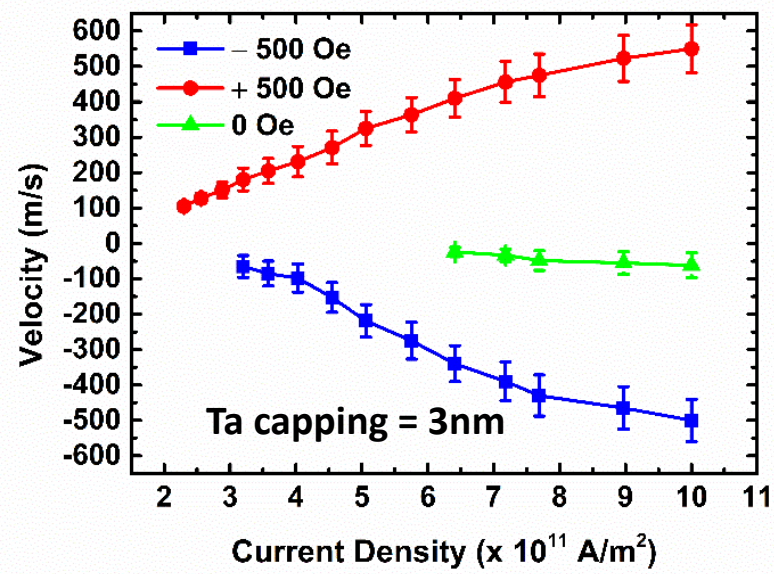

(d)

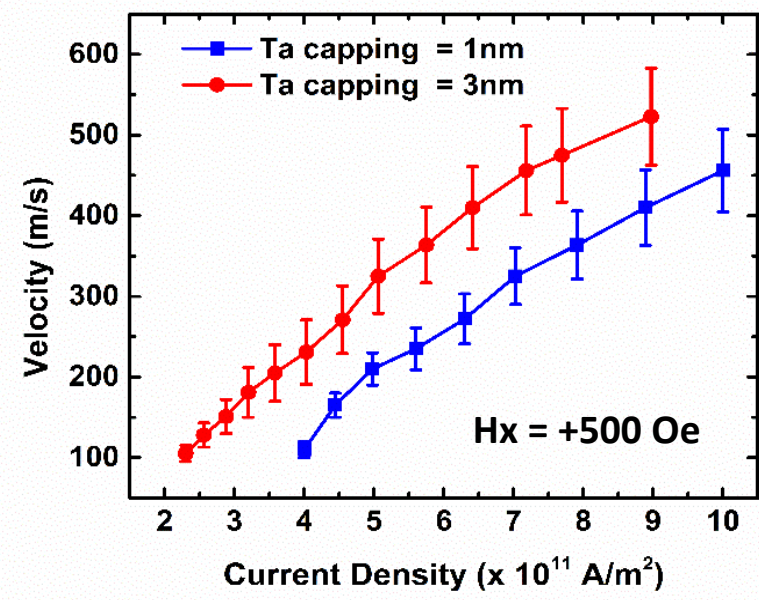

Figure A4-1. Down-up domain wall (DW) velocity versus current density in the presence of fixed magnetic field along and against $x$-direction (a) Ta capping thickness $=1 \mathrm{~nm}$. (b) Ta capping thickness $=3 \mathrm{~nm}$. (c,d) Comparison of velocities for Ta thicknesses $1 \mathrm{~nm}$ and $3 \mathrm{~nm}$ respectively, (c) magnetic field along $-x$-direction (d) magnetic field along $+x$-direction. 
(a)

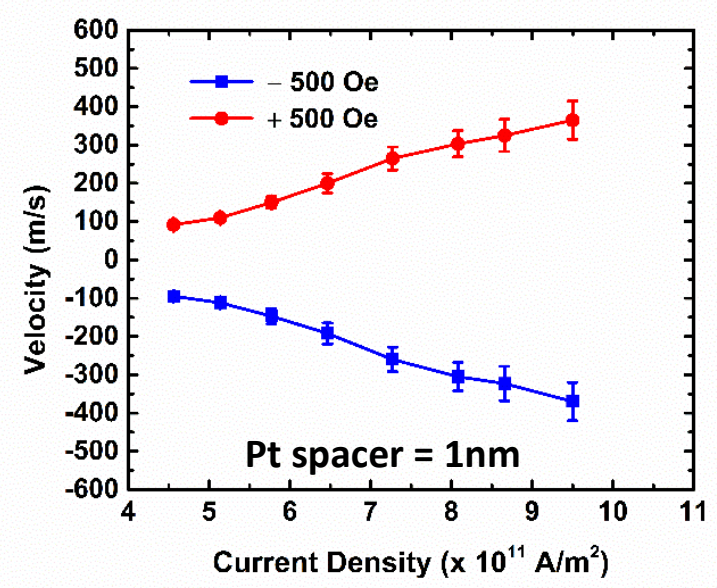

(b)

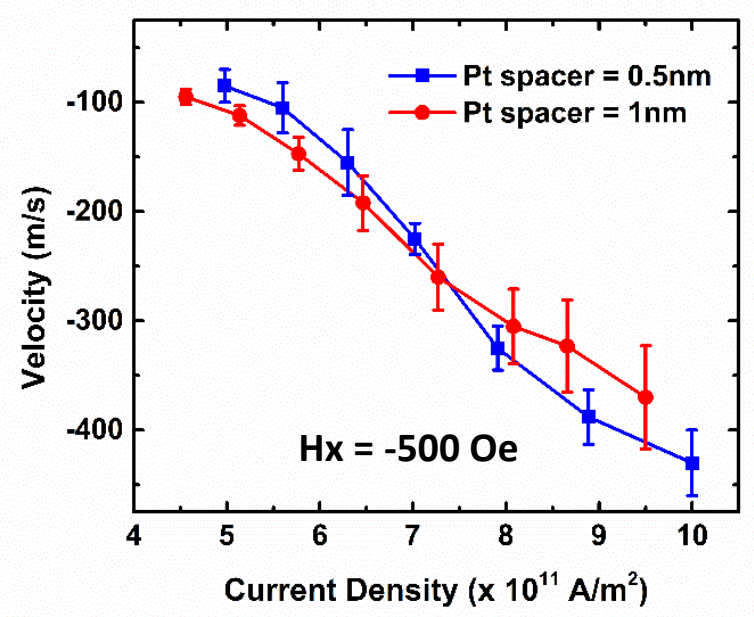

(c)

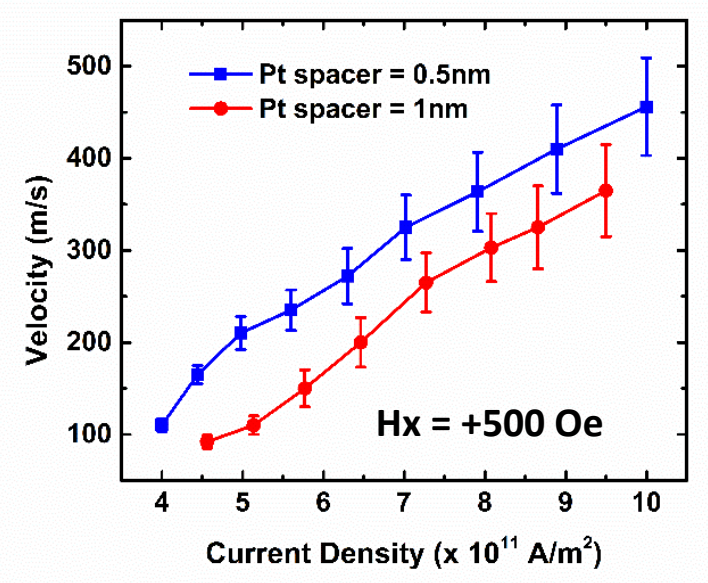

Figure A4-2. Down-up domain wall (DW) velocity versus current density in the presence of fixed magnetic field along and against $x$-direction (a) Pt spacer thickness $=1 \mathrm{~nm}$. (b-c) Comparison of velocities for Pt spacer thicknesses $0.5 \mathrm{~nm}$ and $1 \mathrm{~nm}$ respectively, (b) magnetic field along $-x$-direction (c) magnetic field along $+x$-direction.

\section{A5. Stray field of permanent bar magnet}

The bar magnet used in our study is a permanent magnet cylindrical in shape. The magnetization is primarily along the horizontal direction. We have simulated the magnetic field lines using COMSOL tool. Figure A5 (a) below shows the field lines. The vertical component of the magnetic field is very weak near its axis. Figure A5 (b) is the magnified view with the 
device placed along the axis of the bar magnet. The stray field experienced by the device along the vertical direction is very less.

(a)

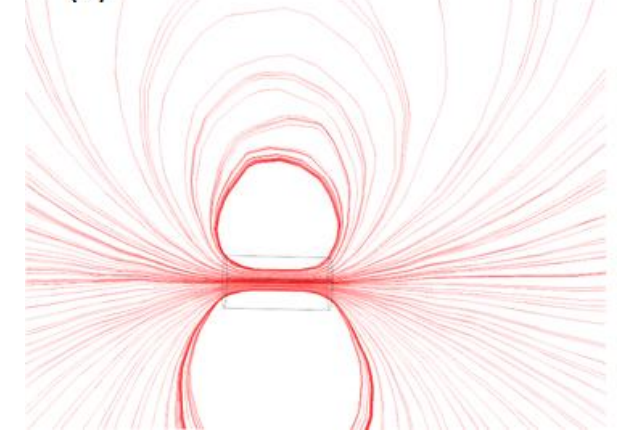

(b)

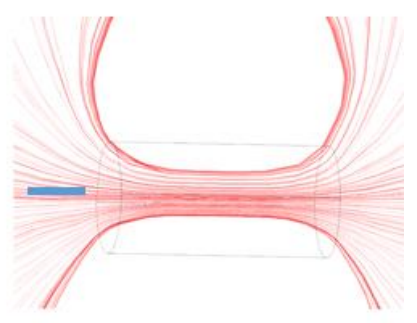

Figure A5. (a) Field lines due to a permanent cylindrical bar magnet. (b) The device, placed along the axis of the bar magnet experiences field along the horizontal direction, the stray field along the vertical direction is weak.

\section{A6. Calculation of Dzyloshinskii-Moriya interaction energy}

Dzyaloshinskii-Moriya interaction (DMI) energy density, D, can be estimated from the DMI field, $H_{D M I}$, using the simple relation, $H_{D M I}=D /\left(\mu_{0} M_{S} \Delta\right)[4,5]$, where $M_{S}$ is the saturation magnetization and $\Delta$ is the domain wall width, given by $\Delta=\left(\mathrm{A} / \mathrm{K}_{\mathrm{eff}}\right)^{1 / 2}$, A and $\mathrm{K}_{\mathrm{eff}}$ being exchange stiffness parameter and effective anisotropy constant respectively. $\mathrm{H}_{\mathrm{DMI}}$ was estimated for two different thin film stacks in section 6.5. Labelling the first thin film stack Ta (3)/Pt (3)/Co (0.7)/Pt (0.5)/Co (0.7)/Ta (1) as stack A and the second thin film stack $\mathrm{Ta}(3) / \mathrm{Pt}$ (3)/Co (0.7)/Pt (1)/Co (0.7)/Ta (1) as stack B. Stack B has Pt spacer thickness which is double of that of stack A.

First consider stack A. From A2, $\mathrm{M}_{\mathrm{S}}=800 \mathrm{emu} / \mathrm{cc}=800 \times 10^{3} \mathrm{~A} / \mathrm{m} . \mathrm{K}_{\mathrm{eff}}=1.6 \times 10^{6} \mathrm{ergs} / \mathrm{cc}$ $=1.6 \times 10^{5} \mathrm{~J} / \mathrm{m}^{3}$. The exchange stiffness can be considered as $\mathrm{A}=18 \mathrm{pJ} / \mathrm{m}$ [3]. Thus $\Delta=10.6$ $\mathrm{nm} . \mathrm{H}_{\mathrm{DMI}}=420$ Oe. Thus $\mathrm{D}=0.35 \mathrm{~mJ} / \mathrm{m}^{2}$.

Next consider stack B. From A2, $\mathrm{M}_{\mathrm{S}}=820 \mathrm{emu} / \mathrm{cc}=820 \times 10^{3} \mathrm{~A} / \mathrm{m} . \mathrm{K}_{\mathrm{eff}}=1.23 \times 10^{6} \mathrm{ergs} / \mathrm{cc}$ $=1.23 \times 10^{5} \mathrm{~J} / \mathrm{m}^{3} \cdot \Delta=12.1 \mathrm{~nm} . \mathrm{H}_{\mathrm{DMI}}=200$ Oe. Thus $\mathrm{D}=0.19 \mathrm{~mJ} / \mathrm{m}^{2}$. 


\section{A7. Reliability and endurance of domain wall based devices}

The domain wall based devices are inherently radiation hard, non-volatile and ideally possess infinite endurance. They are expected to have retention over 10 years if the $\mathrm{K}_{\mathrm{U}} \mathrm{V}>40 \mathrm{k} \mathrm{T}$ [6], where $\mathrm{K}_{\mathrm{U}}$ is the anisotropy constant, $\mathrm{V}$ is the magnetic volume, $\mathrm{k}_{\mathrm{B}}$ is the Boltzmann constant and $\mathrm{T}$ is the temperature.

The reliability studies on DW based devices have been carried out by a group in our laboratory [7]. Read and write efficiencies were compared pre and post radiation. The devices were irradiated by proton beams of $25.3 \mathrm{MeV}$ energy and $5 \times 10^{12} \mathrm{p} / \mathrm{cm}^{2}$ areal flux. The devices were found to retain their magnetic properties and the Hall resistance values.

The data retention can be quantified by noting the probability of switching between bits 0 and 1 via thermal fluctuations, the switching probability is given by the following relation,

$$
P \propto \exp \left(\frac{-E_{G}}{k_{B} T}\right)
$$

The devices are expected to retain data over a period of 10 years at operating temperatures between $80{ }^{\circ} \mathrm{C}$ to $120{ }^{\circ} \mathrm{C}$. To test this, a number of devices are injected with DWs and their position is noted using Kerr imaging. They are then subjected to high temperatures by baking in an oven. The final position of the domains in the devices are compared with initial images and any deviation is attributed to thermal drift. The error rate would give the probability of switching. Substituting in equation (1), a thermal stability factor can be estimated. The value was estimated approximately equal to 34 , indicating reasonable retentivity.

\section{A8. Geometrical asymmetry induced by steepness in branches}

The domain wall (DW) trajectory as demonstrated in Chapter 3 would still be deterministic if the branches have different slopes. The DW would propagate along the branch which is less steep, since the depinning energy barrier would be higher along the branch with steeper slope. 
To validate this argument, simulations were performed using object oriented micromagnetic framework (OOMMF). The results are shown below.

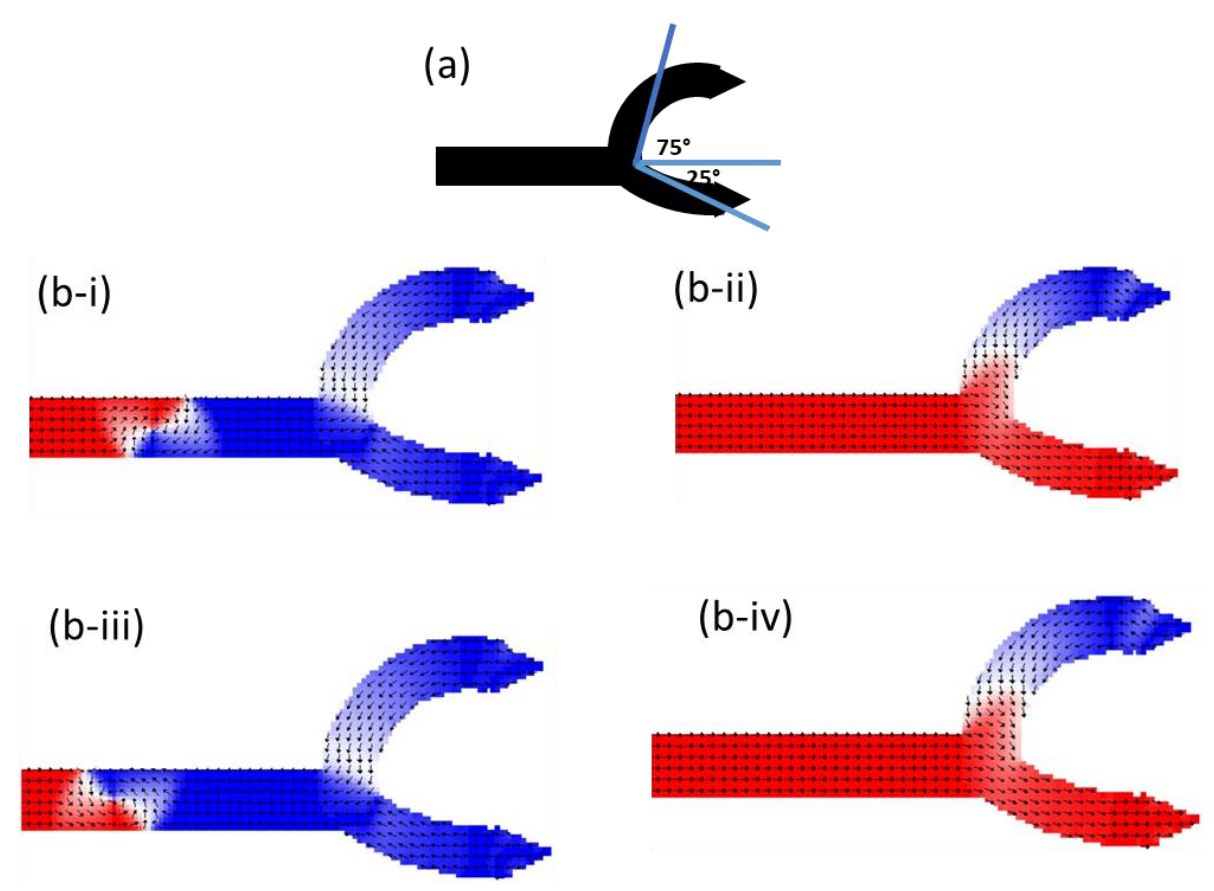

Figure A6 (a) Schematic of U-branch structure with upper branch having steeper slope. (b) Snapshots of simulated configurations (i) initial magnetization configuration when clockwise domain wall is driven in the branch, (ii) final magnetization configuration depicting propagation of domain wall along lower branch (less steep), (iii) initial magnetization configuration when anti-clockwise domain wall is driven in the branch, (ii) final magnetization configuration depicting propagation of domain wall along lower branch (less steep).

Consider the case when upper branch has steeper slope as compared to the lower branch. Figure A6(a) depicts the schematic of one such configuration where the angle subtended by tangent to the upper branch is $75^{\circ}$ and that subtended by lower branch is $25^{\circ}$. Figure $1(\mathrm{~b}-\mathrm{i}-\mathrm{ii})$, shows the initial and final simulated configurations when clockwise vortex DW is driven in the branch and propagated along the lower branch. Figure A6 (b-iii-iv) shows the initial and final simulated configurations when anti-clockwise vortex DW is driven in the branch and propagated along the lower branch. Thus this structure behaves like a 'pull-up' structure. When 
the lower branch has steeper slope, the trend reverses and the DW propagates along the upper branch as shown in Figure A7. This structure behaves like a 'pull-down' structure.
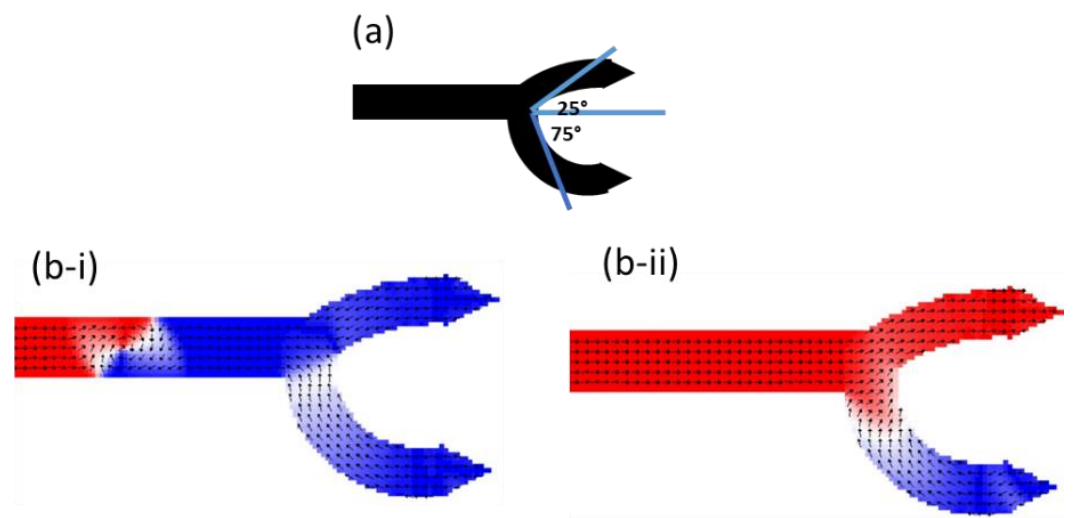

(b-iii)

(b-iv)
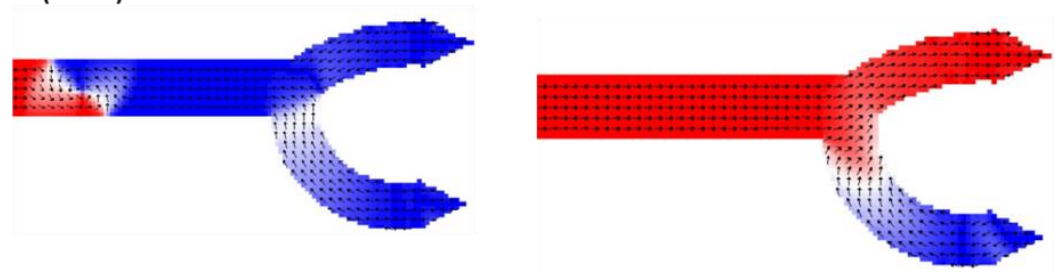

Figure A7 (a) Schematic of U-branch structure with lower branch having steeper slope. (b) Snapshots of simulated configurations (i) initial magnetization configuration when clockwise domain wall is driven in the branch, (ii) final magnetization configuration depicting propagation of domain wall along upper branch (less steep), (iii) initial magnetization configuration when anti-clockwise domain wall is driven in the branch, (ii) final magnetization configuration depicting propagation of domain wall along upper branch (less steep). 


\section{Bibliography}

[1] A. Vansteenkiste and B. Van de Wiele, "MUMAX: A new high-performance micromagnetic simulation tool," Journal of Magnetism and Magnetic Materials, vol. 323, pp. 2585-2591, Nov 2011.

[2] A. Vansteenkiste, J. Leliaert, M. Dvornik, M. Helsen, F. Garcia-Sanchez, and B. Van Waeyenberge, "The design and verification of MuMax3," Aip Advances, vol. 4, Oct 2014.

[3] P. J. Metaxas, J. P. Jamet, A. Mougin, M. Cormier, J. Ferre, V. Baltz, et al., "Creep and flow regimes of magnetic domain-wall motion in ultrathin $\mathrm{Pt} / \mathrm{Co} / \mathrm{Pt}$ films with perpendicular anisotropy," Phys Rev Lett, vol. 99, p. 217208, Nov 232007.

[4] S. G. Je, D. H. Kim, S. C. Yoo, B. C. Min, K. J. Lee, and S. B. Choe, "Asymmetric magnetic domain-wall motion by the Dzyaloshinskii-Moriya interaction," Physical Review B, vol. 88, Dec 22013.

[5] R. Lavrijsen, D. M. F. Hartmann, A. van den Brink, Y. Yin, B. Barcones, R. A. Duine, et al., "Asymmetric magnetic bubble expansion under in-plane field in $\mathrm{Pt} / \mathrm{Co} / \mathrm{Pt}$ : Effect of interface engineering," Physical Review B, vol. 91, Mar 172015.

[6] L. Thomas, G. Jan, S. Le, and P.-K. Wang, "Quantifying data retention of perpendicular spintransfer-torque magnetic random access memory chips using an effective thermal stability factor method," Applied Physics Letters, vol. 106, p. 162402, 2015.

[7] S. Krishnia, "Current driven domain wall dynamics in coupled ferromagnetic structures," Ph. D. Thesis, Nanyang Technological University, 2017. 


\section{List of Publications}

\section{Peer-reviewed journals}

1. P. Sethi, S. Krishnia, W. L. Gan, F. N. Kholid, F. Tan and W. S. Lew, "Bi-directional high speed domain wall motion in perpendicular magnetic anisotropy $\mathrm{Co} / \mathrm{Pt}$ double stack structures”, Sci. Rep. 7, 4964 (2017).

2. S. Krishnia, P. Sethi, W. L. Gan, F. N. Kholid, R. Maddu, T. S. Herng, J. Ding, I. Purnama, and W. S. Lew, "Role of RKKY torque on domain wall motion in synthetic antiferromagnetic nanowires with opposite spin Hall angles”, Sci. Rep. 7, 11715 (2017).

3. S. Krishnia, P. Sethi, W. L. Gan, Q. Y. Wong, G. J. Lim and W. S. Lew, "Spin-orbit torque induced effective field modulation in synthetic antiferromagnetic structure", submitted.

4. P. Sethi, S. Krishnia, S. H. Li and W. S. Lew, "Modulation of spin-orbit torque efficiency by thickness control of heavy metal layers in Co/Pt multilayers", J. Mag. Mag. Mater. 426, 497 (2017).

5. C. Murapaka*, P. Sethi*, S. Goolaup and W. S. Lew, "Reconfigurable logic via gate controlled domain wall trajectory in magnetic network structure", Sci. Rep. 6, 20130 (2016) (*authors contributed equally)

6. P. Sethi, C. Murapaka, S. Goolaup, Y. J. Chen, S. H. Leong and W. S. Lew, "Direct observation of deterministic domain wall trajectory in magnetic network structures", Sci. Rep. 6, 19027 (2016).

7. P. Sethi, C. Murapaka, G. J. Lim and W. S. Lew, "In-plane current induced domain wall nucleation and its stochasticity in perpendicular magnetic anisotropy Hall cross structures", Appl. Phys. Lett. 107, 192401 (2015).

8. Y. J. Chen, H. Yang, S. H. Leong, K. M. Cher, J. F. Hu, P. Sethi, and W. S. Lew, "Erasure temperature measurements of heat assisted magnetic recording media", J. Appl. Phys. 117, 17D117 (2015).

9. C. Murapaka, P. Sethi, S. Goolaup, R. Maddu, Y. J. Chen, S. H. Leong, and W. S. Lew, "Direct observation of domain wall evolution at a bifurcation in magnetic network structures”, Appl. Phys. Exp. 7, 113003 (2014). 


\section{Patents}

1. P. Sethi, C. Murapaka, A. Basu and W. S. Lew, "Magnetic random number generator", Singapore Provisional Patent PAT/285/15/16/SG PRV (2016). US Application No. $15 / 411,811$.

2. W. S. Lew, C. Murapaka, I. Purnama, S. Goolaup, P. Sethi, and C. K. Guite, "Nonvolatile logic device", US Patent US 9,431,599 B2

\section{Conference Presentations}

1. P. Sethi, S. Krishnia, W. L. Gan, F. N. Kholid, R. Maddu, Y. J. Chen, S. H. Leong and W. S. Lew, "Spin-orbit torque induced high speed domain wall motion in $\mathrm{Co} / \mathrm{Pt}$ dual stack", Oral presentation, $61^{\text {st }}$ Annual Conference on Magnetism and Magnetic Materials (MMM 2016), New Orleans, USA*

2. P. Sethi, S. Krishnia, S. H. Li, Y. J. Chen, S. H. Leong, and W. S. Lew, "Highly efficient magnetization switching by spin-orbit torque in magnetic multi-layers", Oral presentation, $61^{\text {st }}$ Annual Conference on Magnetism and Magnetic Materials (MMM 2016), New Orleans, USA.

3. P. Sethi, C. Murapaka, and W. S. Lew, "In-plane current induced domain wall nucleation and its stochasticity in perpendicular anisotropy Hall cross structures", Poster presentation, 2016 Joint MMM-Intermag Conference, San Diego, USA.

4. P. Sethi, C. Murapaka, S. Goolaup, and W. S. Lew, "Programmable logic device by controlling domain wall trajectory in magnetic network structures", Poster presentation, 2016 Joint MMM-Intermag Conference, San Diego, USA.

5. P. Sethi, M. Chandrasekhar, S. Goolaup and W. S. Lew, "Direct observation of deterministic domain wall trajectory in magnetic network structures", Poster presentation, International Conference on Magnetism 2015, Barcelona, Spain.

6. P. Sethi, C. Murapaka, S. Goolaup and W. S. Lew, "Deterministic motion of domain wall in artificial spin-ice lattice", Oral Presentation, Intermag 2015, Beijing, China.**

7. P. Sethi, C. Murapaka, S. Gooalup, R. Maddu, and W. S. Lew, "Transverse domain wall chirality detector", Poster presentation, Intermag 2014, Dresden, Germany.

(*) Best student presentation award nominated, MMM Conference, New Orleans, 2016

(**) Silver award for poster presentation at IEEE Summer School, Minnesota 2015 